\title{
Search for Sterile Neutrinos Using the MiniBooNE Beam
}

\author{
Michel Sorel
}

Submitted in partial fulfillment of the

requirements for the degree

of Doctor of Philosophy

in the Graduate School of Arts and Sciences

COLUMBIA UNIVERSITY

2005 
(c) 2005

Michel Sorel

All Rights Reserved 


\section{ABSTRACT \\ Search for Sterile Neutrinos Using the MiniBooNE Beam}

Michel Sorel

The possibile existence of light sterile neutrinos in Nature is motivated, and the prospects to extend sterile neutrino searches beyond current limits is substantiated, using the MiniBooNE neutrino beam and detector at Fermilab. We report on the neutrino flux predictions for the MiniBooNE experiment, on the characterization of the charged-current, quasi-elastic interactions of muon neutrinos $\left(\nu_{\mu} n \rightarrow \mu^{-} p\right)$ observed, and on the experiment's sensitivity to sterile neutrinos via muon neutrino disappearance. 



\section{Contents}

\begin{tabular}{|ll}
\hline Acknowledgments & vi
\end{tabular}

$\begin{array}{lll}1 & \text { Introduction } & 1\end{array}$

1.1 Neutrinos and the Weak Interactions: a Historical Perspective . . . . 1

1.2 Theory of Massless and Massive Neutrinos . . . . . . . . . . . . . 6

1.2.1 Massless Neutrinos and the Standard Model . . . . . . . . . . 6

1.2 .2 The See-Saw Mechanism for Massive Neutrinos . . . . . . . . 8

1.3 Neutrino Mixing and Flavor Oscillations . . . . . . . . . . . . . 10

$1.4 \quad$ Experimental Signatures for Neutrino Oscillations . . . . . . . . . . . 14

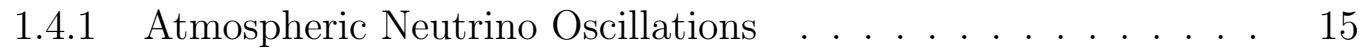

1.4 .2 Solar Neutrino Oscillations . . . . . . . . . . . . . . . 16

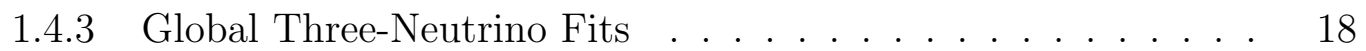

1.4.4 Short-Baseline Neutrino Oscillations and the LSND Signal . . 19

1.5 Other Neutrino Mass and Mixing Measurements . . . . . . . . . . . . 22

1.5.1 Laboratory-Based Neutrino Mass Searches . . . . . . . . . . . 22

1.5.2 Neutrinos and Cosmology . . . . . . . . . . . . . . . 24

1.6 The MiniBooNE $\nu_{\mu} \rightarrow \nu_{e}$ Search . . . . . . . . . . . . . . . . . . . 27

1.7 Synopsis of this Thesis . . . . . . . . . . . . . . . . . . . . 28 
\begin{tabular}{|ll}
2 & Phenomenology of Sterile Neutrinos \\
\hline
\end{tabular}

$2.1 \quad$ Supernova Neutrino Experiments and the Neutrino Mass Hierarchy . 31

2.2 Short-Baseline Neutrino Experiments and the Number of Neutrino Species . . . . . . . . . . . . . . . . . . . . . . 40

$2.3 \quad$ Short-Baseline Neutrino Experiments and Leptonic CP-Violation. . . 57

2.3.1 Including CP-Violation in the Neutrino Oscillation Formalism 58

2.3 .2 Analysis Method . . . . . . . . . . . . . . . . 60

2.3 .3 Results . . . . . . . . . . . . . . . . . . . 62

3 The MiniBooNE Neutrino Beam and the Magnetic Focusing Horn 71

3.1 Overview of the MiniBoonE Neutrino Beam . . . . . . . . . . . . . . 71

3.1.1 The $8 \mathrm{GeV}$ Neutrino Beamline and the Primary Proton Beam 72

3.1 .2 Target Hall and the Secondary Meson Beam . . . . . . . . . . 74

$3.1 .3 \quad$ Decay Region and the Tertiary Neutrino Beam. . . . . . . . . 76

3.2 The MiniBooNE Neutrino Focusing Horn . . . . . . . . . . . . . . . . 77

3.2.1 Horn Physics-Driven Requirements and Design . . . . . . . . . 77

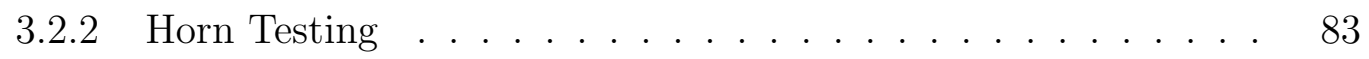

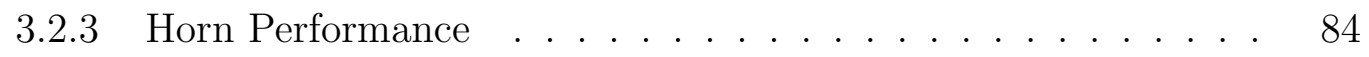

\begin{tabular}{|lll}
4 & MiniBooNE Neutrino Flux Predictions & 89
\end{tabular}

4.1 The Beam Monte Carlo Simulation Code . . . . . . . . . . . . . . . . 89

$4.1 .1 \quad$ Inputs to Neutrino Flux Predictions . . . . . . . . . . . . . . . 89

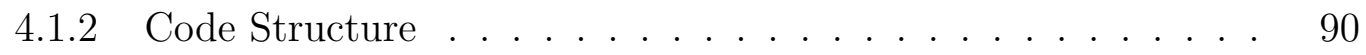

$4.2 \quad$ Simulation of Beamline Geometry, Materials, and Proton Beam . . . 91 
4.2 .1 Geometry and Materials . . . . . . . . . . . . . . . . 91

4.2 .2 Primary Proton Event Generator $\ldots \ldots \ldots$

4.3 Properties of Typical Neutrinos Reaching the MiniBooNE Detector . 95

$4.4 \quad$ Hadronic Interactions . . . . . . . . . . . . . . . . . . . . . . . . . . . . . . . 99

4.4 .1 Inelastic Interactions of Primary Protons in the MiniBooNE Target . . . . . . . . . . . . . . . . . . . . 99

$4.4 .2 \quad$ Other Inelastic Interactions $\ldots \ldots \ldots$

4.4 .3 Elastic Interactions . . . . . . . . . . . . . . . . . . 106

$4.5 \quad$ Electromagnetic Processes $\ldots \ldots \ldots$. . . . . . . . . . . . . 107

4.5.1 Particle Trajectories in the MiniBooNE Horn Magnetic Field . 107

$4.5 .2 \quad$ Multiple Coulomb Scattering _ . . . . . . . . . . . . . . 109

$4.5 .3 \quad$ Ionization by Charged Hadrons $\ldots \ldots \ldots \ldots$

$4.6 \quad$ Neutrinos from Meson and Muon Decays . . . . . . . . . . . . . . . 112

$4.6 .1 \quad$ Decays into Muon Neutrinos . . . . . . . . . . . . . . 115

4.6 .2 Decays into Electron Neutrinos $\ldots \ldots \ldots \ldots \ldots$

4.7 Neutrino Flux Predictions at the MiniBooNE Detector $\ldots \ldots \ldots$

5 Overview of Neutrino Interactions in the $1 \mathrm{GeV}$ Energy Regime 122

5.1 The NUANCE Cross-Section Generator . . . . . . . . . . . . . . . 124

5.2 The Free Nucleon Quasi-Elastic Interaction . . . . . . . . . . . 127

5.3 Nuclear Effects . . . . . . . . . . . . . . . . . . . . . . . 133

$5.3 .1 \quad$ Pauli Suppression . . . . . . . . . . . . . . . . . . . . . . 134

5.3 .2 Fermi Momentum . . . . . . . . . . . . . . . . . . . 136

5.3 .3 Final State Interactions $\ldots \ldots \ldots \ldots$ 
6.1 Overview of the MiniBooNE Detector Hardware . . . . . . . . . . . . 142

6.1.1 The Nuclear Target: MiniBooNE Mineral Oil . . . . . . . . . 143

6.1 .2 Optical Readout: MiniBooNE Photomultiplier Tubes . . . . . 144

$6.1 .3 \quad$ Data Acquisition System and Trigger . . . . . . . . . . 146

$6.1 .4 \quad$ Calibration Devices . . . . . . . . . . . . . . . . . . . . . 149

6.2 The MiniBooNE Detector Response . . . . . . . . . . . . . . . . . . 151

$6.2 .1 \quad$ Light Production . . . . . . . . . . . . . . . . . . . . . . 151

6.2 .2 Light Transmission . . . . . . . . . . . . . . . . . . 155

$6.2 .3 \quad$ PMT/DAQ Response, and Hit-Level Detector Calibration . . 158

6.3 Reconstruction and Particle Identification Algorithms in MiniBooNE 166

6.3 .1 Event Reconstruction . . . . . . . . . . . . . . . 166

6.3.2 Reconstruction-Level Detector Calibration and Validation . . 170

6.3 .3 Particle Identification $\ldots \ldots \ldots \ldots \ldots$

7 Muon Neutrino Charged-current, Quasi-Elastic Interactions in Mini-

BooNE

175

7.1 Description of the Event Selection and Neutrino Energy Reconstruction 176

7.1.1 Hit-Level and Reconstruction-Level Selection . . . . . . . . . 177

7.1.2 Fisher Discriminant Method and Event-Level Selection . . . . 179

7.1.3 Description of the Neutrino Energy Reconstruction Method . 183

$7.2 \quad$ Predicted Event Selection and Neutrino Energy Reconstruction Performance . . . . . . . . . . . . . . . . . 186

$7.2 .1 \quad$ Efficiency and Purity in Selecting CCQE Interactions. . . . . 186 
$7.2 .2 \quad$ Neutrino Energy Reconstruction . . . . . . . . . . . . . . . . . 191

7.3 Validation of the Event Selection and Energy Reconstruction with MiniBooNE Data . . . . . . . . . . . . . . . . . . . . . . . . . . . . 192

$7.3 .1 \quad$ Calibration Samples . . . . . . . . . . . . . . . . 193

7.3 .2 The CCQE Sample . . . . . . . . . . . . . . . . . . . . 196

7.4 CCQE Results . . . . . . . . . . . . . . . . . . . . . . . 199

8 The Muon Neutrino Disappearance Analysis 205

8.1 The Oscillation Fitting Code . . . . . . . . . . . . . . . . . . . . . . 206

8.2 Systematic Errors Assumptions ～. . . . . . . . . . . . . . . . . 207

8.2 .1 Neutrino Flux . . . . . . . . . . . . . . . . . . . . . . 209

8.2 .2 Neutrino Cross-sections . . . . . . . . . . . . . . . . . . . . . . 210

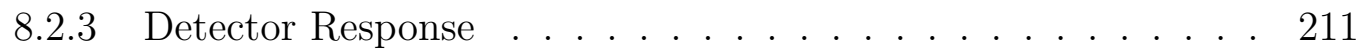

$8.3 \quad$ Sensitivity to $\nu_{\mu} \rightarrow \nu_{\mu}$ Oscillations . . . . . . . . . . . . 212

8.4 Comparing the CCQE Results with Predictions . . . . . . . . . . . . 215

8.5 Toward Muon Neutrino Disappearance Results . . . . . . . . . . . . . 219

$\begin{array}{ll}\text { Conclusions } & 222\end{array}$

\begin{tabular}{ll}
\hline Bibliography & 223
\end{tabular} 


\section{Acknowledgments}

It seems a lifetime since I started working on the MiniBooNE experiment... During these years, the experiment's design was finalized, the experiment has been built, and now has been taking data for two and a half years! The office space for the collaboration moved from its spartan original location at the Fermilab Lab-E trailers, to the more "corporate" cubicles of the Fermilab High Rise building. Many new people arrived, and others have left. This thesis would not exist (or would be a far worse one) without mentioning all the "MiniBooNErs" who taught me so much during these years, and whose company I have enjoyed greatly.

The person I am more indebted to is certainly Janet Conrad, my advisor. You typically only get one Ph.D. advisor in life, and I was very fortunate that Janet has been my advisor. Janet is a great physicist, and also particularly talented in recognizing and putting into good use the different human potentials of the persons she directs. Thank you, Janet, for your unconditional and generous support, your intuition and pragmatism in solving my research difficulties, and the inspiring motivation that you have given me: those have guided and helped me throughout these years. I would like to see MiniBooNE, "her experiment", turning into a great success, if nothing else because Janet has invested a lot of energy into it, and she fully deserves it.

The Columbia University neutrino group is a workhorse of the MiniBooNE experiment, with involvments in almost all aspects of the experiment. Much of this is due to Mike Shaevitz, the group leader. Thank you, Mike, for all your intelligent 
suggestions, for sharing your knowledge and experience in neutrino physics with me, and for your common sense. The person I have worked the most closely with during these years is Jocelyn Monroe, graduate student in the Columbia group. Thank you Jocelyn, this thesis contains a lot of your efforts as well! I am sure that Jocelyn will turn the analysis described here into a much better one. I cannot remember how many cigarettes I have smoked here at Fermilab with Dave Schmitz. Thank you, Dave, for the many enlightening and fun discussions we had. Alexis Aguilar is also a graduate student in the Columbia neutrino group. Thank you, Alexis, for your willingness to help, and for your positive and humble attitude. Sam Zeller and Jon Link have been the post-doctoral fellows of the group I have overlapped the most. Thank you, Sam, for your effectiveness and scientific rigor. Thank you, Jon, for your guidance, sanguine personality, and humor.

Having spent more than four years at Fermilab, I had the privilege to interact with many other MiniBooNE collaborators, not affiliated with Columbia University. Jen Raaf (from U. of Cincinnati) and I had parallel careers in graduate school, and shared many long days in our common office. Thank you, Jen, for your kindness and generosity; one more month and you will finish! Bill Louis, from Los Alamos, is the co-spokesperson of the experiment, together with Janet. Thank you, Bill, for your scientific intuitions, and your extremely supportive attitude. Morgan Wascko is a post-doc at LSU, and the MiniBooNE detector calibration "guru". Thank you, Morgan, for providing me with countless information used in this thesis. Steve Brice, from Fermilab, is the MiniBooNE analysis coordinator. Thank you, Steve, for your clarity, and for teaching me many useful things. The other "MiniBooNErs" I would especially like to thank are: Yong Liu, Ion Stancu, Mike Wilking, Len Bugel, Linda Coney, Bruce Brown, Ioanis Kourbanis, Panagiotis Spentzouris, Ray Stefanski, Chris Cox, Gerry Garvey, Andrew Green, Chris Green, Gordon McGregor, Geoff Mills, Richard Van de Water, Bill Metcalf, Byron Roe, Peter Meyers, Ryan Patterson, Hiro Tanaka, Eric Hawker, Alessandro Curioni, and Bonnie Fleming. 
Many thanks to Lalla Grimes, Nicole Griggs, Ann Therrien, and Elaine Schmidt, to make my extended stay far away from the Columbia University campus and from Nevis Laboratories so smooth.

Finally, I would like to extend my warm thanks to some of the persons outside of the work sphere, whose support and friendship have been equally important to me during these studies. First, to my girlfriend. Olga, hopefully I will now stop pronouncing words like "Monte Carlo" and "kaon decay" during my sleep! Second, to my friends at Fermilab and Chicago, in particular to: Lorenzo, Ludovic, Paul, Jovan, Enrique, and Veronika. Third, to my parents, my brother, and to my italian grand-mother, whose motto I will remember: "meglio un asino vivo che un dottore morto" (approximate translation: "it is better to be alive and uncultivated, rather than dead and with a Ph.D. degree"). 


\section{Chapter 1}

\section{Introduction}

\subsection{Neutrinos and the Weak Interactions: a His- torical Perspective}

The history of the neutrino dates back to the very early stages of the Universe, a small fraction of a second after the Big Bang, but this elementary particle is known to mankind only since 1930. That year, W. Pauli postulated the existence of a neutral particle to explain the continuous energy spectrum of electrons in the $\beta$-decay of radioactive nuclei, via (as we know today) $n \rightarrow p+e^{-}+\bar{\nu}_{e}$, where $n, p, e^{-}, \bar{\nu}_{e}$ indicate a neutron, proton, electron, and electron antineutrino, respectively.

After the discovery of the neutron in 1932 by J. Chadwick [1], E. Fermi coined the word "neutrino" to describe this "little, neutral particle", in contrast to the more massive neutron. In 1932, C. D. Anderson discovered the positron [2, the antiparticle of the electron, confirming the prediction of Dirac's relativistic theory of quantum mechanics. In 1934, E. Fermi proposed the first theory of weak interactions [3], successfully describing nuclear $\beta$-decay processes. This interaction operates at short, nuclear ranges, and it is termed "weak" in contrast to the nuclear strong interaction, binding protons and neutrons together in nuclei, which was inferred by J. Chadwick 
in 1921. In 1937, E. Majorana first speculated that the hypothetical neutrinos might behave differently than Dirac particles, and that neutrinos and antineutrinos might be the same entity [4].

Neutrinos from $\beta$-decay were first detected in 1956 by C. Cowan and F. Reines [5], from the decay of neutron-rich nuclei produced in the fission of heavy elements at nuclear reactors. Electron antineutrinos were observed via the detection of the positron emitted in inverse $\beta$-decay, $\bar{\nu}_{e} p \rightarrow e^{+} n$, and the subsequent $\gamma$ emission from the neutron capture. The very small interaction rate for this neutrino interaction measured by Cowan and Reines was in rough agreement with Fermi's theory, and confirmed the weak character of neutrino interactions.

After the detection of the neutrino, efforts to try to understand neutrino properties, in particular its mass and spin, began. In 1956, from an analysis of weak particle decays available at the time, Lee and Yang concluded that weak interactions (unlike electromagnetic and strong nuclear interactions) do not conserve parity, or equivalently that the formalism describing a process mediated by the weak force is not invariant under a mirror reflection of the physics system under consideration [6]. The year after, C. S. Wu and collaborators unambiguously confirmed parity violation in weak interactions [7].

The non-conservation of parity led Lee and Yang to formulate, in 1957, a "twocomponent theory" of massless neutrinos [8], in which the half-integer spin neutrinos can only have one possible helicity state, that is only one possible spin orientation with respect to their momentum direction vector, as opposed to the two helicity states allowed for the other particles known in nature. The helicity $H$ of the neutrino was determined to be left-handed in 1958, by Goldhaber et al. [9], that is $H \equiv \vec{p} \cdot \vec{S} /|\vec{p}|=-\hbar / 2$, where $\vec{p}$ is the three-momentum vector, $\vec{S}$ the neutrino spin. Parity violation was formally embedded into a new theory of weak interactions, called the (V-A) theory, in that same year, by E. C.G. Sudarshan, R. E. Marshak, and others [12]. 
During the same period, in 1957, B. Pontecorvo realized that if the neutrino is a massive particle and lepton number is not conserved, then it may oscillate over time (or, equivalently, distance) into its own antiparticle, the antineutrino [10]. Pontecorvo was not aware of distinct neutrino flavors at the time, and this first oscillation theory was developed in analogy to strangeness oscillations in the neutral kaon system, postulated by Gell-Mann and Pais two years before [11].

Distinct neutrino flavors came as a surprise, when the muon neutrino was discovered in 1962 with the first accelerator-based neutrino beam, by L. Lederman, M. Schwartz, J. Steinberger, and collaborators [13]. This neutrino beam, consisting of a beam of muon neutrinos from pion decays in flight, triggered the exploration of neutrino interactions of significantly higher energies than what was previously achievable. Soon after the discovery of the muon neutrino, Z. Maki, M. Nakagawa and S. Sakata considered the possibility of neutrino oscillations among the electron and the muon flavor states, and introduced neutrino mixing [14].

Another violation of a fundamental physics symmetry in weak interactions, the CP symmetry, was discovered in 1964 by J. W. Cronin and V. Fitch, in the neutral kaon system [15]. The CP operator combines the parity operation P mentioned above, with the charge-conjugation operation $\mathrm{C}$, reversing the sign of charge and magnetic moment of a particle, and therefore implying the interchange of particle and antiparticle. In 1973, M. Kobayashi and T. Maskawa realized that, for CP violation to occur, at least three generations of Dirac particles needed to exist [16]. In 1967, A. D. Sakharov first pointed out that CP violation in weak interactions could be related to the matter-antimatter asymmetry present in the Universe today [17]. While the discovery of $\mathrm{CP}$ violation and its possible cosmological connection were derived from the quark sector, it is believed today that they might also be applicable to the neutrino sector.

In the 1960s, the gauge theory of electroweak interactions as we know it today was developed by S. L. Glashow, S. Weinberg and A. Salam [18]. Important aspects 
of the electroweak theory are the unification of the weak and electromagnetic interactions, the prediction of neutral weak interactions, and the spontaneous symmetry breaking via the Higgs mechanism [19], generating masses for the gauge vector bosons mediating the charged and neutral weak interactions. The neutral current weak interaction of neutrinos, characterized by a neutrino in its final state (and not a charged lepton, as for charged weak interactions), was first detected in 1973 by the Gargamelle bubble chamber experiment at CERN [20]. The massive, charged and neutral, gauge vector bosons postulated by the electroweak theory were first observed by the UA1 experiment at the CERN SPS collider in 1983 [21].

Also during the 1960s, the exploration of the nuclear fusion processes in the core of the Sun via the weakly-interacting neutrinos began. In 1968, R. Davis and collaborators first measured a deficit of solar neutrinos compared to the predictions of the Solar Standard Model, with the Homestake chlorine detector [22]. In the following years, the detection of solar neutrinos was firmly established, but the deficit with respect to expectations remained. Already in 1968, V. N. Gribov and B. Pontecorvo proposed neutrino oscillations among the two types of neutrinos known at the time, the electron and the muon neutrino, as a possible mechanism to explain the solar neutrino deficit [24].

In the mid-1980s, large water Cherenkov detectors were built underground to measure the decay of the proton predicted by grand unified theories. While proton decay has not been observed to date, two major discoveries were made with these detectors, related to the observation of two other extraterrestrial sources of neutrinos. First, in 1987, the Kamiokande (Japan) and IMB (USA) detectors simultaneously observed a burst of neutrinos from the SN1987A supernova explosion in the Large Magellanic Cloud [25]. Second, in 1988, the Kamiokande experiment measured a deficit in the number of muon neutrinos produced by the interactions of cosmic rays in the Earth's atmosphere [26]. The muon neutrino deficit anomaly was later confirmed and interpreted in terms of muon neutrino to tau neutrino oscillations by the 
Super-Kamiokande experiment, in 1998 [27]. This third type of neutrino, the tau neutrino, was directly observed only in 2000 by the DONUT experiment at Fermilab [28]. However, its existence was postulated since the discovery in 1975 of its electrically charged counterpart, the tau lepton, by M. Perl and collaborators [29], and indirectly observed in 1989 via precision electroweak measurements at the LEP electron-positron collider at CERN [30].

After the discovery of the solar neutrino deficit by the Homestake chlorine experiment, the exploration of solar neutrinos continued on both the theoretical and experimental fronts. In the late 1970s and early 1980s, S. P. Mikheev, A. Y. Smirnov and L. Wolfenstein formulated neutrino oscillations in the presence of dense matter, predicting large matter effects in solar neutrino oscillations [31. In the early 1980s and early 1990s, the Kamiokande, SAGE, and GALLEX underground experiments confirmed the solar neutrino deficit [33], by detecting neutrinos mostly originated in different solar nuclear fusion reactions than the ones detected by the Homestake chlorine experiment. In 2001, the Super-Kamiokande and SNO experiments unambiguosuly confirmed that solar neutrino oscillations among the three neutrino flavors occur, and that matter effects are present in solar neutrino oscillations [36].

In the 1990s, the trend in experimental neutrino oscillation physics switched back to the use of man-made neutrino sources, from reactors and particle accelerators. Several neutrino experiments studying oscillations were performed, many with short neutrino propagation pathlengths between the neutrino source and the neutrino detection locations, roughly between $10 \mathrm{~m}$ and $1 \mathrm{~km}$. No oscillation signals were seen at short baselines, with the exception of a possible indication for muon antineutrino to electron antineutrino oscillations in the accelerator-based LSND experiment at Los Alamos [37], first reported in 1996, and still unconfirmed nor excluded by other experiments. The consequences of this result are the focus of this thesis. During the same period, long baseline neutrino experiments detected neutrinos produced hundreds of km away from accelerator and reactor sources. In 2002, the K2K 
accelerator-based experiment confirmed atmospheric neutrino oscillations [38], and the KamLAND reactor-based experiment confirmed solar neutrino oscillations [39].

This concludes the historical account of major experimental discoveries and ideas in particle physics and astrophysics that are closely related to neutrinos and the weak interactions. Among the scientific advances mentioned above, ten have already been awarded with Nobel Prizes in physics.

\subsection{Theory of Massless and Massive Neutrinos}

\subsubsection{Massless Neutrinos and the Standard Model}

The Standard Model gauge theory of electroweak interactions describe the interactions of neutrinos in terms of the exchange of $W^{ \pm}, Z^{0}$ intermediate vector bosons. The couplings $W \nu l$ and $Z^{0} \nu \nu$ describe charged-current and neutral-current interactions, respectively, where $l$ refer to charged leptons. In this theory, neutrinos and antineutrinos are massless, and only left-helicity neutrino states $\nu_{L}$ and right-helicity antineutrino states $\bar{\nu}_{R}$ exist, that is weak interactions involve only two out of the four components of Dirac fields. The chiral left-handed helicity states are given by $\nu_{L} \equiv\left[\left(1-\gamma^{5}\right) / 2\right] \nu$, where $\gamma^{5} \equiv i \gamma^{0} \gamma^{1} \gamma^{2} \gamma^{3}$ and $\gamma^{\mu}$ are Dirac matrices, and correspond, for massless neutrinos, to the helicity states defined in the previous Section. The experimentally observed (V-A) structure of charged-current weak interactions, or equivalently the fact that left-handed neutrinos couple only to left-handed charged leptons, is enforced into the theory by the $S U(2)_{L}$ gauge symmetry requirement for the charged-current interaction Lagrangian:

$$
-\mathcal{L}_{C C}=\frac{g}{\sqrt{2}} W_{\rho}^{-} \overline{l_{L}} \gamma^{\rho} \nu_{L}+\text { h.c. }
$$


where $g$ is the electroweak coupling constant, $\overline{l_{L}} \equiv l_{L}^{\dagger} \gamma^{0}$, and $L$ indicates the weak isospin doublet, $I=1 / 2$ :

$$
L \equiv\left(\begin{array}{l}
\nu \\
l
\end{array}\right)
$$

with $I_{3}=1 / 2,-1 / 2$ for $\nu$ and $l$, respectively, in analogy to the proton-neutron isospin doublet in strong interactions. The right-handed charged lepton $l_{R}$ forms a weak-isospin singlet, $I=0$. Similarly, the Lagrangian describing neutral current interactions is given by:

$$
-\mathcal{L}_{N C}=\frac{g}{2 \cos \theta_{W}} Z_{\rho}^{0} \overline{\nu_{L}} \gamma^{\rho} \nu_{L}+\text { h.c. }
$$

where $\theta_{W}$ is the Weinberg (weak mixing) angle.

The conservation of electric charge and electric current is ensured by the invariance of the $\mathcal{L}_{C C}+\mathcal{L}_{N C}$ Lagrangian under $U(1)_{Y}$, where the weak hypercharge $Y$ is related to the electric charge $Q$ and the third component of the weak isospin via $Q=I_{3}+Y / 2$.

The Standard Model electroweak theory assumes three lepton generations, $\alpha=$ $e, \mu, \tau$. This theory assumes an accidental flavor symmetry that is not imposed by any gauge invariance principle, and that preserves the individual $L_{e}, L_{\mu}, L_{\tau}$ lepton quantum numbers in all weak interactions, where the lepton quantum numbers are defined as $L_{e}=1, L_{\mu}=L_{\tau}=0$ for the $\left(\nu_{e}, e\right)_{L}$ weak doublet, and similarly for the muon and tau doublets. The corresponding antiparticles have opposite lepton numbers. Therefore, neutrino flavor oscillations are not allowed in this theory.

Finally, the gauge invariance of the weak interaction Lagrangian is spontaneously broken, and all fermions (with the exception of neutrinos) and $W, Z$ gauge bosons acquire mass, via the Higgs mechanism. In this mechanism, a weak isospin doublet of scalar fields is added to the theory, and charged lepton masses are obtained via Yukawa interaction terms in the Lagrangian:

$$
-\mathcal{L}_{D, l}=f_{l}\left(\overline{\nu_{L}} \phi^{+} l_{R}+\overline{l_{L}} \phi^{0} l_{R}\right)+\text { h.c. }
$$


where $f_{l}$ are dimensionless Yukawa couplings and $\phi^{+, 0}$ are the two components of the Higgs weak isospin doublet, with electric charge +1 and zero, respectively. After spontaneous symmetry breaking, $\left\langle\phi^{+}\right\rangle=0,\left\langle\phi^{0}\right\rangle=v / \sqrt{2}$ where $v \simeq 246 \mathrm{GeV}$ is the vacuum expectation value of the Higgs field, the neutrinos remain massless, while the charged leptons acquire a mass $m_{D, l}=f_{l} v / \sqrt{2}$. The Yukawa mass term for the lepton $l,-\mathcal{L}_{D, l}=m_{D, l} \bar{l}_{L} l_{R}+$ h.c., is called a Dirac mass term. The same mechanism applies for the generation of quark fermion masses. The Yukawa couplings are free parameters of the theory, and set by the experimentally measured fermion masses $m$ via $f \simeq 5.7 \cdot 10^{-6}(\mathrm{~m} / 1 \mathrm{MeV})$.

\subsubsection{The See-Saw Mechanism for Massive Neutrinos}

Neutrino mass terms can be added to the Standard Model Lagrangian in two ways. The first way is in direct analogy to the Dirac masses of quarks and charged leptons, by adding the two extra components of the Dirac neutrino field, the right-handed neutrino and the left-handed antineutrino fields:

$$
-\mathcal{L}_{D}=\overline{\nu_{L}} m_{D} \nu_{R}+\text { h.c. }
$$

Majorana mass terms can be constructed from the left-handed neutrino states alone, from right-handed neutrino states only, or from both. For example, for righthanded neutrino states only, the Majorana mass term is:

$$
-\mathcal{L}_{R}=\frac{1}{2} \overline{\left(\nu_{R}\right)^{c}} m_{R} \nu_{R}+\text { h.c. }
$$

where $\nu^{c}=C \bar{\nu}^{T}, C$ is the charge-conjugation operator, and $m_{R}$ is a free parameter with dimensions of mass. Majorana mass terms in the Lagrangian convert particles into their own antiparticles, and are therefore forbidden for all electrically charged fermions because of charge conservation. Moreover, processes involving Majorana mass terms violate the Standard Model total lepton number $L \equiv L_{e, \mu, \tau}$ by two units, which is not a good quantum number anymore. 
Neutrino masses, although not measured yet, are known to be small, of the order of $1 \mathrm{eV}$ or less [60]. The explanation of neutrino masses via Dirac mass terms alone require neutrino Yukawa couplings of the order of $10^{-12}$ or less. The current theoretical prejudice is that neutrino Yukawa couplings with $f_{\nu} \ll 1$ and $f_{\nu} \ll f_{l}$ are unnatural, if not unlikely.

The so-called "see-saw mechanism" provides a way to accommodate neutrino masses that is considered more "natural". The simplest realization of the see-saw model is to add both a Dirac mass term and a right-handed mass term to the Lagrangian, as given by Eqs. 1.5 and 1.6, for each of the three neutrino flavors. For each neutrino flavor, two fields of definite chirality and definite mass are obtained. Assuming neutrino Yukawa couplings of the order of the charged fermion couplings, and $m_{R} \gg v \gtrsim m_{D}$ of the order of some high mass scale where new physics responsible for neutrino masses is supposed to reside, the see-saw mechanism yields a small left-handed neutrino mass eigenvalue of $m_{i} \simeq m_{D}^{2} / m_{R}$ and a large right-handed neutrino mass eigenvalue of $m_{3+i} \simeq m_{R}$, where $i=1,2,3$ runs over the three fermion generations of the Standard Model. The corresponding mass eigenfields $\nu_{i}$ and $\nu_{3+i}$ are found to be equal to their corresponding charge-conjugate fields, $\nu_{i}^{c}=\nu_{i}$ and $\nu_{3+i}^{c}=\nu_{3+i}$. Particles described by fields satisfying this condition are called Majorana particles. Majorana particles and Majorana antiparticles are identical, and are truly neutral particles in the sense that they do not have electrical nor any other type of charge. Neutrinos whose fields do not satisfy this Majorana condition are called Dirac neutrinos. In the Standard Model case of left-handed neutrino fields only, there is no physical difference between massless Majorana neutrinos and massless Dirac neutrinos, while the Dirac/Majorana nature of neutrinos is in principle observable in the case of massive neutrinos.

The neutrino couplings to the $W$ and $Z$ bosons are unaffected by Dirac and/or Majorana mass terms in the Lagrangian, and therefore only the left-handed, active, neutrinos participate in weak interactions, while their right-handed partners don't, 
and are called sterile neutrinos.

\subsection{Neutrino Mixing and Flavor Oscillations}

As discussed above, neutrinos only interact via weak processes. Experimentally, this implies that neutrinos are both produced and detected as weak eigenstates. In contrast, the free propagation of neutrinos between their production and detection point is governed by the free Hamiltonian, whose eigenstates are states with definite neutrino mass. Nothing requires that neutrino weak and mass eigenstates coincide. In the case in which a weak eigenstate is expressed by a mixture (linear combination) of more than one mass eigenstate, and the neutrino mass eigenvalues in this mixture are different from each other, oscillations among neutrino flavors can be observed as a function of propagation time/distance.

Neutrinos of a given weak flavor $\alpha=e, \mu, \tau$ are defined as the neutrinos produced or detected in association with the charged lepton $l_{\alpha}$, in the charged weak interactions described by:

$$
-\mathcal{L}_{C C}=\frac{g}{\sqrt{2}} W_{\rho}^{-} \sum_{\alpha=e, \mu, \tau} \overline{l_{L \alpha}} \gamma^{\rho} \nu_{L \alpha}+\text { h.c. }
$$

In a straightforward generalization of the simplest see-saw mechanism presented above to include three generations, the three weak eigenstates $\left|\nu_{\alpha}\right\rangle$ are generally expressed in terms of a linear combination of six eigenstates $\nu_{i}(t)$ :

$$
\left|\nu_{\alpha}\right\rangle=U_{\alpha i}^{*}\left|\nu_{i}\right\rangle
$$

where $\alpha=e, \mu, \tau, i=1, \ldots, 6$, and $U$ is a $6 \times 6$ unitary mixing matrix diagonalizing the $6 \times 6$ symmetric, generally complex neutrino mass matrix $M_{D+R}$ in the weak basis, via the unitary transformation $U^{T} M_{D+R} U=M_{\text {diag }}$, where $M_{D+R}$ is now specified in terms of the $3 \times 3$ matrix blocks $m_{D}$ and $m_{R}$ :

$$
M_{D+R}=\left(\begin{array}{ll}
0 & \left(m_{D}\right)^{T} \\
m_{D} & m_{R}
\end{array}\right)
$$


From Eq. 1.8 and from the time evolution of free neutrinos, the propagation of a neutrino produced as weak eigenstate $\alpha$ at time $t$ and distance $L$ is:

$$
\left|\nu_{\alpha}(t)\right\rangle=\sum_{k} U_{\alpha k}^{*} e^{-i\left(E_{k} t-p_{k} L\right)}\left|\nu_{k}\right\rangle=\sum_{\beta=e, \mu, \tau}\left(\sum_{k} U_{\alpha k}^{*} e^{-i\left(E_{k} t-p_{k} L\right)} U_{\beta k}\right)\left|\nu_{\beta}\right\rangle
$$

Therefore, the probability to detect a neutrino of weak eigenstate $\beta$, after a time $t$ from its production as weak eigenstate $\alpha$, is:

$$
P_{\nu_{\alpha} \rightarrow \nu_{\beta}}(t)=\left|\left\langle\nu_{\beta} \mid \nu_{\alpha}(t)\right\rangle\right|^{2}=\left|\sum_{k} U_{\alpha k}^{*} e^{-i\left(E_{k} t-p_{k} L\right)} U_{\beta k}\right|^{2}
$$

If neutrinos are ultrarelativistic (and $c \equiv 1$ ), then $t \simeq L$, and furthermore if neutrinos are produced with definite energy $E$, then $E-p_{k} \simeq m_{k}^{2} /(2 E)$. By reabsorbing the phase $e^{-i\left(E-p_{1}\right) L}$, Eq. 1.11 can be rewritten in the ultrarelativistic regime as:

$$
P_{\nu_{\alpha} \rightarrow \nu_{\beta}}(t)=\left|\sum_{i} U_{\alpha i}^{*} e^{-i \Delta m_{i 1}^{2} L /(2 E)} U_{\beta i}\right|^{2}
$$

where we have defined $\Delta m_{i 1}^{2} \equiv m_{i}^{2}-m_{1}^{2}$. By developing the product, using simple trigonometric identities, and reintroducing $\hbar, c$, Eq. 1.13 can be recast into the form [40]:

$$
\begin{aligned}
P\left(\nu_{\alpha} \rightarrow \nu_{\beta} ; L, E\right)=\delta_{\alpha \beta} & -4 \sum_{i>j} \mathcal{R}\left(U_{\alpha i}^{*} U_{\beta i} U_{\alpha j} U_{\beta j}^{*}\right) \sin ^{2}\left[\Delta m_{i j}^{2} L /(4 \hbar c E)\right]+ \\
& +2 \sum_{i>j} \mathcal{I}\left(U_{\alpha i}^{*} U_{\beta i} U_{\alpha j} U_{\beta j}^{*}\right) \sin \left[\Delta m_{i j}^{2} L /(2 \hbar c E)\right]
\end{aligned}
$$

where $\mathcal{R}$ and $\mathcal{I}$ indicate the real and imaginary parts, respectively. For antineutrinos, the oscillation probability is obtained from Eq. 1.13 by replacing the mixing matrix $U$ with its complex-conjugate matrix. Therefore, if the mixing matrix is not real, neutrino and antineutrino oscillation probabilities can differ. Furthermore, from Eq. 1.13, it follows directly that neutrino flavor oscillations $(\alpha \neq \beta)$ are possible only if neutrino weak and mass eigenstates do not coincide (non-diagonal neutrino mixing matrix $U)$ and neutrino mass eigenvalues are not all degenerate $\left(\Delta m_{i j}^{2} \neq 0\right.$ for at least one $(i, j)$ pair). Moreover, Eq. 1.13 shows that, at least in the CP-conserving case, neutrino oscillations in vacuum are not sensitive to the sign of the mass splittings 
$\Delta m_{i j}^{2}$. In very dense matter environments, the neutrino propagation cannot be considered as a free propagation, and matter effects modify Eq. 1.13 in a way that is not symmetric under the change $\Delta m_{i j}^{2} \rightarrow-\Delta m_{i j}^{2}$. A special case of neutrino oscillations in matter will be considered in Chapter 2 .

If no specific assumptions on the physics mechanisms that generate neutrino masses and mixings are made, how many independent parameters need to be measured, experimentally? Considering $N$ neutrino species, there are $N$ independent neutrino mass parameters. In neutrino oscillation experiments, there are $N-1$ independent mass splittings, for example $\Delta m_{i, i-1}^{2}, i=2, \ldots, N$. Also, a (generally complex) $N \times N$ mixing matrix contains $N^{2}$ independent, real parameters. These can be subdivided into $N(N-1) / 2$ mixing angles and $N(N+1) / 2$ phases. In the case of Dirac neutrinos, there are $(N-1)(N-2) / 2$ physical phases, all of which can be in principle detected in oscillation experiments. In the case of Majorana neutrinos, there are $N-1$ additional physical, Majorana phases. Majorana phases, unlike Dirac phases, cannot be measured in neutrino oscillation experiments, but can, in principle, be detected in different experiments (see Section 1.5).

The expression given above, with its $6 \times 6$ neutrino mixing matrix, represents the general case for Majorana neutrinos produced via a see-saw model. In this general case, the three sterile neutrino states can be described in terms of the same mixing matrix $U$ (its last three rows) and the same six mass eigenstates as the three active states. Therefore, both oscillations among active neutrino flavors and active-sterile neutrino oscillations are in principle observable [41]. Oscillations only among active flavors are observable in two, more commonly considered, cases, and a $3 \times 3$ mixing matrix involving only three neutrinos is sufficient. The first case is the one in which sterile neutrinos are very heavy, $m_{R}>v$ where $v$ is the electroweak energy scale, as typically assumed. In this case, the mixings $U_{\alpha i}$, with $i>3$, are greatly suppressed, by factors proportional to $m_{D} / m_{R}$. The second case is the one of Dirac neutrinos from a minimally extended Standard Model theory, that is neutrinos whose masses 
are generated by the addition of a Dirac mass term only in the Lagrangian. In this case, sterile neutrinos decouple from the theory, and are not physically observable. We will give some examples of the phenomenology of active-sterile neutrino oscillations in Chapter 2, while below we consider two important special cases: active three-neutrino, and active two-neutrino mixings. We focus on neutrino oscillations phenomenology, and we drop Majorana phases from the discussion.

The neutrino pathlengths, $L$, and energies, $E$, probed in neutrino experiments are such that, often, Eq. 1.13 can be recast in terms of a single mass splitting. This limit is sometimes called the "one mass scale dominance" limit, or the "quasi-two neutrino mass approximation". By making the further assumption that only two neutrino weak eigenstates $(\alpha, \beta=e, \mu$ for definiteness) and two mass eigenstates $(i=1,2)$ are relevant, Eq. 1.13 can be written as:

$$
\begin{array}{rl}
P\left(\nu_{\alpha} \rightarrow \nu_{\beta}\right)=\delta_{\alpha \beta}-4 & \mathcal{R}\left(U_{\alpha 2}^{*} U_{\beta 2} U_{\alpha 1} U_{\beta 1}^{*}\right) \sin ^{2}\left[\Delta m^{2} L /(4 \hbar c E)\right]+ \\
+ & 2 \mathcal{I}\left(U_{\alpha 2}^{*} U_{\beta 2} U_{\alpha 1} U_{\beta 1}^{*}\right) \sin \left[\Delta m^{2} L /(2 \hbar c E)\right]
\end{array}
$$

where $\Delta m^{2} \equiv \Delta m_{21}^{2}$. From the discussion above, the physical parameters in the $2 \times 2$ mixing matrix are one mixing angle $\theta$ and no Dirac phases. The mixing angle $\theta$ specifies the rotation among weak eigenstates and mass eigenstates:

$$
U=e_{\mu}\left(\begin{array}{cc}
1 & 2 \\
\cos \theta & \sin \theta \\
-\sin \theta & \cos \theta
\end{array}\right)
$$

Let us assume now that $\alpha=\mu, \beta=e$. From Eqs. 1.14 and 1.15 , the two-neutrino oscillation probability formula is:

$$
\begin{aligned}
& P\left(\nu_{\mu} \rightarrow \nu_{e}\right)=\sin ^{2} 2 \theta \sin ^{2}\left[\Delta m^{2} L /(4 \hbar c E)\right] \simeq \\
\simeq & \sin ^{2} 2 \theta \sin ^{2}\left[1.27 \Delta m^{2}\left(e V^{2}\right) L(k m) / E(G e V)\right]
\end{aligned}
$$

with $P\left(\nu_{\mu} \rightarrow \nu_{\mu} ; L, E\right)=1-P\left(\nu_{\mu} \rightarrow \nu_{e} ; L, E\right)$.

In the case of three-neutrino mixing, there are two independent mass splittings 
$\Delta m_{21}^{2}, \Delta m_{32}^{2}$, three mixing angles $\theta_{12}, \theta_{13}, \theta_{23}$, and one Dirac phase $\delta$. A common parametrization for the $3 \times 3$ mixing matrix is [40]:

$$
\begin{aligned}
U=\left(\begin{array}{ccc}
1 & 0 & 0 \\
0 & c_{23} & s_{23} \\
0 & -s_{23} & c_{23}
\end{array}\right)\left(\begin{array}{ccc}
c_{13} & 0 & s_{13} e^{-i \delta} \\
0 & 1 & 0 \\
-s_{13} e^{i \delta} & 0 & c_{13}
\end{array}\right)\left(\begin{array}{ccc}
c_{12} & s_{12} & 0 \\
-s_{12} & c_{12} & 0 \\
0 & 0 & 1
\end{array}\right)= \\
=\left(\begin{array}{ccc}
s_{12} c_{13} & s_{13} e^{-i \delta} \\
\left(-s_{12} c_{23}-c_{12} s_{23} s_{13} e^{i \delta}\right) & \left(c_{12} c_{23}-s_{12} s_{23} s_{13} e^{i \delta}\right) & s_{23} c_{13} \\
\left(s_{12} s_{23}-c_{12} c_{23} s_{13} e^{i \delta}\right) & \left(-c_{12} s_{23}-s_{12} c_{23} s_{13} e^{i \delta}\right) & c_{23} c_{13}
\end{array}\right)
\end{aligned}
$$

where $c_{i j} \equiv \cos \theta_{i j}$ and $s_{i j} \equiv \sin \theta_{i j}$

\subsection{Experimental Signatures for Neutrino Oscilla- tions}

Neutrino oscillations are inferred from a discrepancy between the number of observed neutrino interactions with respect to the number of interactions predicted, assuming no oscillations. Moreover, since the oscillation probability is, in general, affected by the neutrino energy, the extent of this discrepancy is neutrino energy-dependent. Two types of oscillation measurements are possible. The first type is an appearance measurement, where a neutrino of some weak flavor type is produced (say, a muon neutrino), and a different neutrino type (e.g., an electron neutrino) is observed at some distance from the neutrino source. The second type is a disappearance measurement, where a known amount of neutrinos of some weak flavor type is produced, and a smaller amount of that same weak flavor type is observed after some distance/time.

If a discrepancy between observations and predictions assuming no oscillations is present, one can then find what are the possible oscillation hypotheses that are compatible with the observations, if any. 


\subsubsection{Atmospheric Neutrino Oscillations}

The disappearance of muon neutrinos produced by cosmic ray interactions in the atmosphere has now been firmly established using large underground water Cherenkov detectors. Atmospheric neutrinos that can be cleanly reconstructed in these detectors are typically produced with an energy $E \sim 1-10 \mathrm{GeV}$, and travel distances $L \sim 10^{2}-10^{4} \mathrm{~km}$, where the shortest distance corresponds to downward-going neutrinos produced in the atmosphere above the detector, and the longest distance corresponds to upward-going neutrinos produced in the atmosphere at the antipodes and crossing the Earth's diameter, before interacting in the detector. Therefore, these experiments cover the wide range $L / E \sim 1-10^{4} \mathrm{~km} / \mathrm{GeV}$, and neutrino oscillations in the range $\Delta m^{2} \sim 10^{-4}-1 \mathrm{eV}^{2}$ may cause observable $L / E$ spectral distortions. The current-generation, atmospheric water Cherenkov detector is the Super-Kamiokande detector in Japan. The neutrino energy and direction is estimated from the total momentum vector magnitude and direction of final state particles from the neutrino interaction, reconstructed via the Cherenkov ring pattern. The pattern of the most energetic Cherenkov ring is used to distinguish electron neutrino from muon neutrino interactions. The number of observed downward-going muon neutrinos (small $L / E$ ) is consistent with the number expected for no neutrino oscillations, while only about half of upward-going muon neutrino interactions are observed, compared to the no-oscillation hypothesis. Moreover, the number of electron neutrinos observed at all $L / E$ is consistent with the Standard Model expectation. Overall, the Super-Kamiokande data can be explained via $\nu_{\mu} \rightarrow \nu_{\tau}$ oscillations due to full mixing $(\theta \simeq \pi / 4)$ and $\Delta m^{2} \simeq 2.4 \cdot 10^{-3} \mathrm{eV}^{2}$. More stringent limits on $\nu_{\mu} \rightarrow \nu_{e}$ oscillations at this $\Delta m^{2}$ scale have been obtained by the CHOOZ reactor antineutrino experiment, which did not measure any disappearance of few MeV electron antineutrinos over pathlengths of $L \sim 1 \mathrm{~km}$. The ratio of observed-to-predicted $L / E$ distributions for muon neutrino interactions at Super-Kamiokande is given in the left panel of Fig. 1.1, together with the best-fit $\nu_{\mu} \rightarrow \nu_{\tau}$ hypothesis, and two alternative, beyond the Stan- 

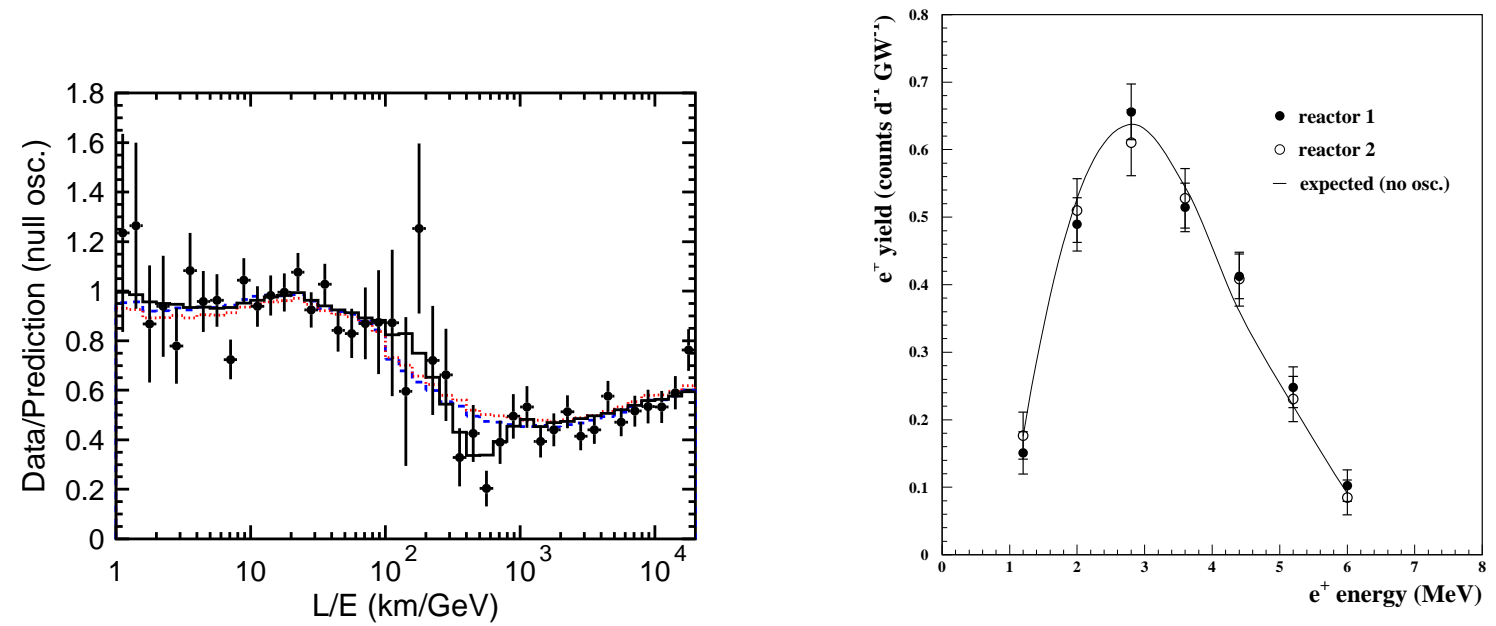

Figure 1.1: Left: points indicate the ratio of observed to predicted (for no oscillations) muon neutrino events at Super-Kamiokande, as a function of the reconstructed $L / E$, together with the best-fit hypotheses for $\nu_{\mu} \rightarrow \nu_{\tau}$ oscillations (solid line), neutrino decay (dashed), and neutrino decoherence (dotted) [42]. Right: points indicate the positron yields from $\bar{\nu}_{e} p \rightarrow$ $e^{+} n$ interactions, as a function of positron energy, observed by the CHOOZ experiment; the solid line show the yield expected for no oscillations 433.

dard Model explanations of neutrino data, neutrino decay and neutrino decoherence, which are disfavored compared to oscillations [42. The observed energy distribution of positron events from $\bar{\nu}_{e} p \rightarrow e^{+} n$ interactions at CHOOZ is given in the right panel of Fig. 1.1, together with the no-oscillation prediction [43]. The Super-Kamiokande atmospheric result has recently been confirmed by the K2K experiment [38], which uses the same Super-Kamiokande detector, and $\sim 1 \mathrm{GeV}$ muon neutrinos from a accelerator-based source located $250 \mathrm{~km}$ away from the detector.

\subsubsection{Solar Neutrino Oscillations}

The most convincing evidence to date that solar electron neutrinos oscillate into other active neutrino flavors $\left(\nu_{\mu}\right.$ or $\left.\nu_{\tau}\right)$ comes from the SNO experiment in Canada. 

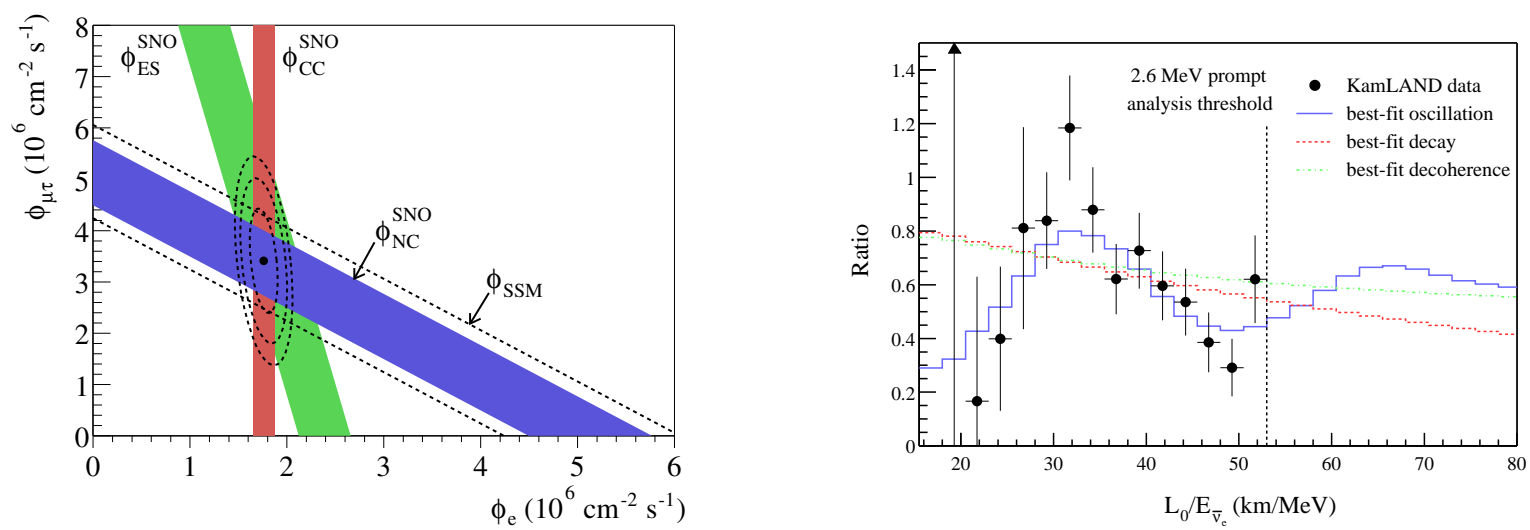

Figure 1.2: Left: solar muon or tau neutrino flux versus electron neutrino flux, as measured by three different neutrino reactions with the SNO detector 44]; the dashed lines enclose the Solar Standard Model prediction [45]. Right: points indicate the $\bar{\nu}_{e}$ spectrum observed in KamLAND, divided by the no-oscillation expectation, as a function of $L_{0} / E$. The curves indicate the best-fit neutrino oscillation, decay, and decoherence hypotheses [46].

The SNO detector is also an underground Cherenkov detector, filled with heavy water $\left(D_{2} O\right)$, and detects few $\mathrm{MeV}$ solar neutrino interactions via the charged-current (CC) process $\nu_{e} d \rightarrow p p e^{-}$, the neutral-current (NC) process $\nu_{\alpha} d \rightarrow p n \nu_{\alpha}$, and the elastic scattering (ES) process $\nu_{\alpha} e^{-} \rightarrow \nu_{\alpha} e^{-}$. While the CC process is only sensitive to electron neutrinos, the $\mathrm{NC}$ and $\mathrm{ES}$ processes are sensitive to all active neutrino flavors $\nu_{\alpha}, \alpha=e, \mu, \tau$. The left panel in Figure 1.2 shows the solar neutrino fluxes $\phi_{\mu, \tau}$ and $\phi_{e}$ estimated by the SNO measurements [44]. The total active neutrino flux $\phi_{\mu, \tau}+\phi_{e}$ is in good agreement with the Solar Standard Model expectation $\phi_{\mathrm{SSM}}[45]$. On the other hand, only about a third of the originally produced electron neutrinos is detected on Earth.

In general, results from various solar neutrino experiments are affected by neutrino oscillations in differing ways, depending on their energy threshold for neutrino detection. By combining the SNO results with the results from the SuperKamiokande, Homestake, and gallium experiments, and by taking into account matter effects inside the Sun and Earth, it is found that solar neutrino oscillations are consistent with $\nu_{e} \rightarrow \nu_{\mu, \tau}$ oscillations specified by a mixing angle $\theta$ with $\tan ^{2} \theta \simeq 0.4$, 
and $\Delta m^{2} \simeq 8 \cdot 10^{-5} \mathrm{eV}^{2}$ [47]. For this $\Delta m^{2}$ range, matter effects play a dominant role on neutrino oscillations, allowing to resolve the $(\theta \leftrightarrow \pi / 2-\theta)$ degeneracy of vacuum neutrino oscillations.

The KamLAND experiment in Japan has confirmed solar neutrino oscillations by detecting the disappearance of few $\mathrm{MeV}$ electron antineutrinos produced at nuclear reactors located a few hundreds of $\mathrm{km}$ away from the liquid scintillator KamLAND detector [39]. Electron antineutrino interactions $\bar{\nu}_{e} p \rightarrow n e^{+}$are tagged by the prompt positron signal, and the $2.2 \mathrm{MeV} \gamma$ ray from the $\sim 200 \mu s$ delayed neutron capture signal. The positron energy is used to estimate the $\bar{\nu}_{e}$ energy $E$ event-by-event, while the average neutrino pathlength $L_{0} \simeq 180 \mathrm{~km}$ is inferred from simulations. The $L_{0} / E$ distribution of KamLAND data is shown in the right panel of Fig. 1.2, together with the best-fit neutrino oscillation, neutrino decay, and neutrino decoherence hypotheses [46]. As for atmospheric neutrinos, the disappearance of reactor antineutrinos is well explained by neutrino oscillations, while mechanisms other than oscillations are highly disfavored.

\subsubsection{Global Three-Neutrino Fits}

Solar and atmospheric neutrino oscillation data can be collectively analyzed. It is found [47] that all these datasets are consistent with oscillations among the three neutrino flavors $\nu_{e}, \nu_{\mu}, \nu_{\tau}$. Furthermore, the mass splittings $\Delta m_{21}^{2}, \Delta m_{32}^{2}$, and the mixing angles $\theta_{12}, \theta_{23}, \theta_{13}$ appearing in Eq. 1.17, describing three-neutrino oscillations are now relatively well known, as shown by the results of a global fit given in Fig. 1.3 [47]. Present data also measure the sign of the solar mass splitting $\Delta m_{21}^{2}$ (or equivalently the $\theta_{12}$ octant). On the other hand, the sign of the atmospheric mass splitting $\Delta m_{32}^{2}$ and the value of the Dirac CP-violating phase $\delta$ are currently unknown. Experiments are now being planned to probe these two parameters, as well as to extend the $\theta_{13}$ sensitivity [48]. 

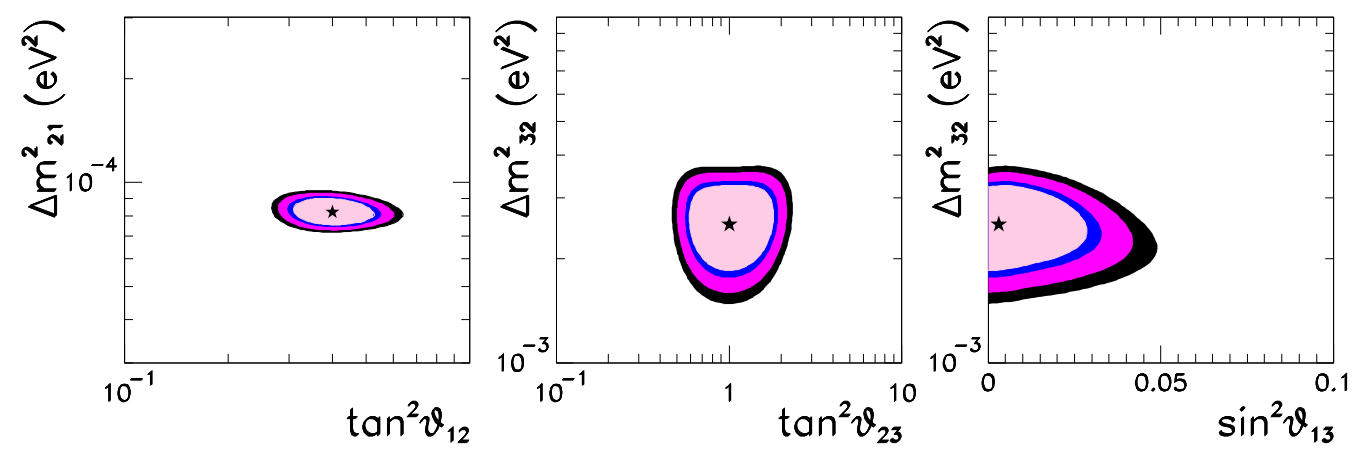

Figure 1.3: Allowed ranges for the three-neutrino mass and mixing parameters $\Delta m_{21}^{2}$, $\Delta m_{31}^{2}, \theta_{12}, \theta_{23}$, and $\theta_{13}$, from global fits of solar and atmospheric oscillation data 47 ]. The regions correspond to 90\%, 95\%, 99\% and $3 \sigma$ confidence level.

\subsubsection{Short-Baseline Neutrino Oscillations and the LSND Signal}

Several neutrino oscillation searches have been performed to probe higher $\Delta m^{2}$ values than what indicated by solar and atmospheric neutrino oscillations. In these searches, the possible appearance or disappearance of neutrinos produced at accelerators and reactors over distances $L \sim 10^{-2}-1 \mathrm{~km}$ is studied, and these experiments are generally called short-baseline experiments. Several oscillation channels have been explored, as summarized in Tab. 1.1.

The only positive oscillation result among the short-baseline oscillation searches is the evidence for $\bar{\nu}_{\mu} \rightarrow \bar{\nu}_{e}$ oscillations reported by the Liquid Scintillator Neutrino Detector (LSND) experiment at Los Alamos Laboratories (USA) [37]. A muon antineutrino source with a known energy distribution with endpoint of $52.8 \mathrm{MeV}$ is produced via the $\pi^{+} / \mu^{+}$decay at rest chain $\pi^{+} \rightarrow \mu^{+} \nu_{\mu}, \mu^{+} \rightarrow e^{+} \nu_{e} \bar{\nu}_{\mu}$, with little $\bar{\nu}_{e}$ contamination. At detection, about $30 \mathrm{~m}$ away from the neutrino source, a $\bar{\nu}_{e}$ excess is seen by tagging the positron and the $\gamma$ from neutron capture from the inverse $\beta$ decay reaction, $\bar{\nu}_{e} p \rightarrow e^{+} n$. The neutrino energy $E_{\nu}$ is estimated from the measured 


\begin{tabular}{|l|l|l|l|l|l|l|}
\hline Channel & Exp. & Optimal & Low $\Delta m^{2}$ & \multicolumn{2}{|c|}{$\sin ^{2} 2 \theta$ Constraint } & Ref. \\
& & $\Delta m^{2}$ & Reach & High $\Delta m^{2}$ & Optimal $\Delta m^{2}$ & \\
\hline$\nu_{\mu} \rightarrow \nu_{e}$ & LSND & $2 \cdot 10^{0}$ & $3 \cdot 10^{-2}$ & {$[2.5-3.8] \cdot 10^{-3}$} & {$[1.2-3.2] \cdot 10^{-3}$} & {$[37]$} \\
& KARMEN & $3 \cdot 10^{0}$ & $6 \cdot 10^{-2}$ & $<1.7 \cdot 10^{-3}$ & $<1.0 \cdot 10^{-3}$ & {$[49]$} \\
& NOMAD & $3 \cdot 10^{1}$ & $4 \cdot 10^{-1}$ & $<1.4 \cdot 10^{-3}$ & $<1.0 \cdot 10^{-3}$ & {$[50]$} \\
\hline$\nu_{e} \rightarrow \nu_{\theta}$ & Bugey & $6 \cdot 10^{-1}$ & $1 \cdot 10^{-2}$ & $<1.4 \cdot 10^{-1}$ & $<1.3 \cdot 10^{-2}$ & {$[51]$} \\
& CHOOZ & $6 \cdot 10^{-3}$ & $7 \cdot 10^{-4}$ & $<1.0 \cdot 10^{-1}$ & $<5 \cdot 10^{-2}$ & {$[43$} \\
\hline$\nu_{\mu} \rightarrow \nu_{\mu}$ & CCFR84 & $9 \cdot 10^{2}$ & $6 \cdot 10^{0}$ & none & $<2 \cdot 10^{-1}$ & {$[52]$} \\
& CDHS & $3 \cdot 10^{0}$ & $3 \cdot 10^{-1}$ & none & $<5.3 \cdot 10^{-1}$ & {$[53]$} \\
\hline$\nu_{\mu} \rightarrow \nu_{\tau}$ & NOMAD & $1 \cdot 10^{2}$ & $7 \cdot 10^{-1}$ & $<3.3 \cdot 10^{-4}$ & $<2.5 \cdot 10^{-4}$ & {$[54]$} \\
& CHORUS & $6 \cdot 10^{1}$ & $5 \cdot 10^{-1}$ & $<6.8 \cdot 10^{-4}$ & $<4.5 \cdot 10^{-4}$ & {$[55]$} \\
\hline$\nu_{e} \rightarrow \nu_{\tau}$ & NOMAD & $1 \cdot 10^{2}$ & $6 \cdot 10^{0}$ & $<1.5 \cdot 10^{-2}$ & $<1.1 \cdot 10^{-2}$ & {$[54]$} \\
& CHORUS & $9 \cdot 10^{1}$ & $7 \cdot 10^{0}$ & $<5.1 \cdot 10^{-2}$ & $<4 \cdot 10^{-2}$ & {$[55]$} \\
\hline
\end{tabular}

Table 1.1: Most sensitive short- and medium-baseline neutrino oscillation searches in various oscillation channels. $\Delta m^{2}$ is expressed in $e V^{2}$, and the low $\Delta m^{2}$ reach and $\sin ^{2} 2 \theta$ constraints are given at the $90 \%$ confidence level.

positron energy and emission angle, using two-body kinematics. The excess has a $3.8 \sigma$ significance, and its $L / E_{\nu}$ dependence is compatible with oscillations, as shown in the left panel of Fig. 1.4. The KARMEN experiment at Rutherford Laboratory (UK) performed a $\bar{\nu}_{\mu} \rightarrow \bar{\nu}_{e}$ search with a neutrino source and detection principle that are similar to the LSND ones [49]. The main differences between the two experiments' oscillation sensitivities are due to the shorter KARMEN baseline of $L=17.7$ $\mathrm{m}$, and the smaller KARMEN statistics. The KARMEN results are compatible with no $\bar{\nu}_{\mu} \rightarrow \bar{\nu}_{e}$ oscillations, and are shown in the right panel of Fig. 1.4 as a function of positron, prompt energy.

The LSND-allowed and KARMEN-excluded regions in two-neutrino oscillation parameter space $\left(\sin ^{2} 2 \theta, \Delta m^{2}\right)$ are shown in the left panel of Fig. 1.5, together with the exclusion region from the null electron antineutrino disappearance result obtained by the Bugey experiment [37, 49, 51]. The KARMEN and Bugey null oscillation re- 

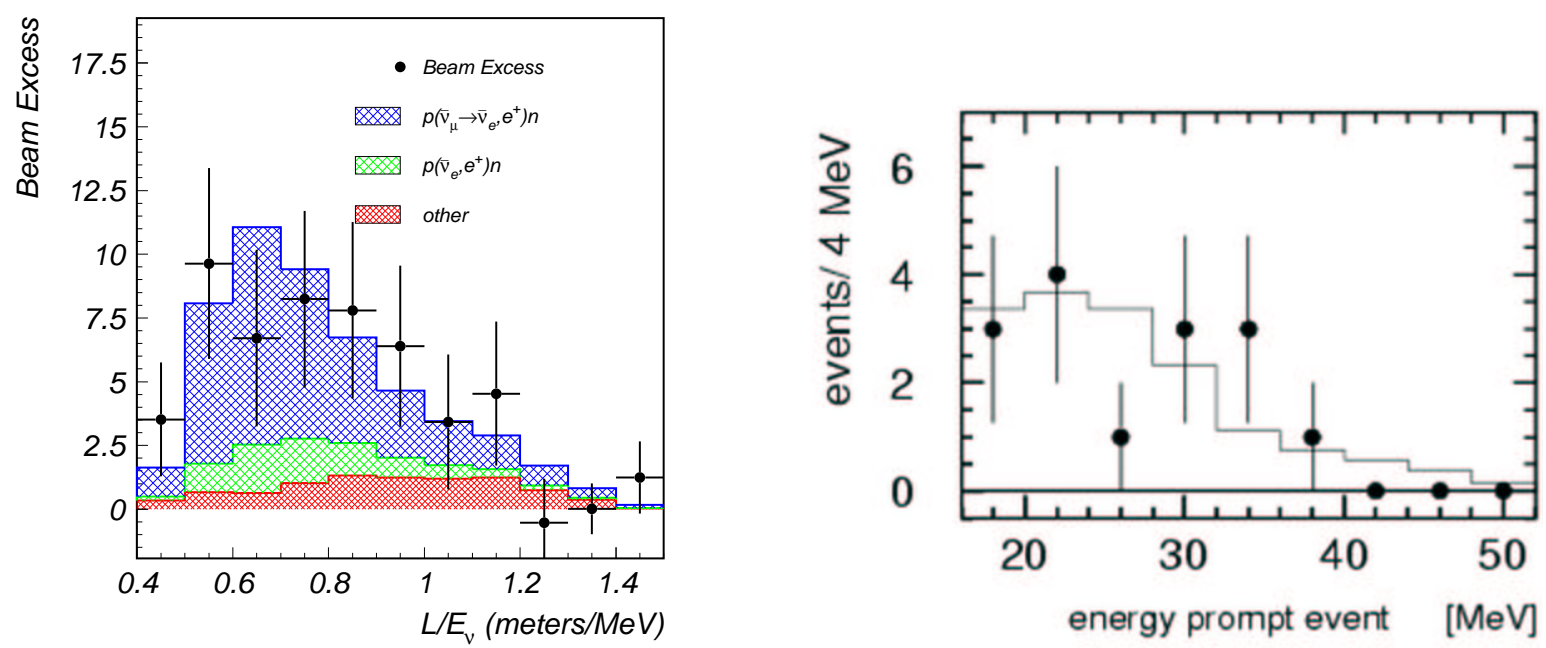

Figure 1.4: Left: the points show the $L / E$ distribution of $\bar{\nu}_{e}$ candidate events observed in LSND; the red and green histograms show the expectations for no oscillations, and the blue histogram show the best-fit oscillation contribution. Right: the points indicate the prompt, visible energy distribution observed in KARMEN, and the histogram shows the expectation for no oscillations.

sults exclude a part of the LSND allowed region, while another part has not yet been refuted nor confirmed by any neutrino experiment other than LSND. A joint LSND-KARMEN oscillation analysis has been performed by collaborators from both experiments [56]. The analysis yields a level of $64 \%$ compatibility of the two experimental outcomes. Assuming statistical compatibility, the joint allowed region in oscillation parameters space, shown in the right panel of Fig. 1.5, is consistent with the LSND-only region.

The LSND oscillation evidence at high $\Delta m^{2}$, in conjunction with the solar and atmospheric neutrino oscillation results, cannot be accommodated within the simplest Standard Model extension possible, that is one with only three, active, massive neutrinos. In simple terms, the reason stems from the fact that $\Delta m_{\mathrm{LSND}}^{2} \gg$ $\Delta m_{\mathrm{atm}}^{2}+\Delta m_{\mathrm{sol}}^{2}$, in contrast with the expectation from three-neutrino oscillation models, that is $\Delta m_{31}^{2} \equiv \Delta m_{32}^{2}+\Delta m_{21}^{2}$. 

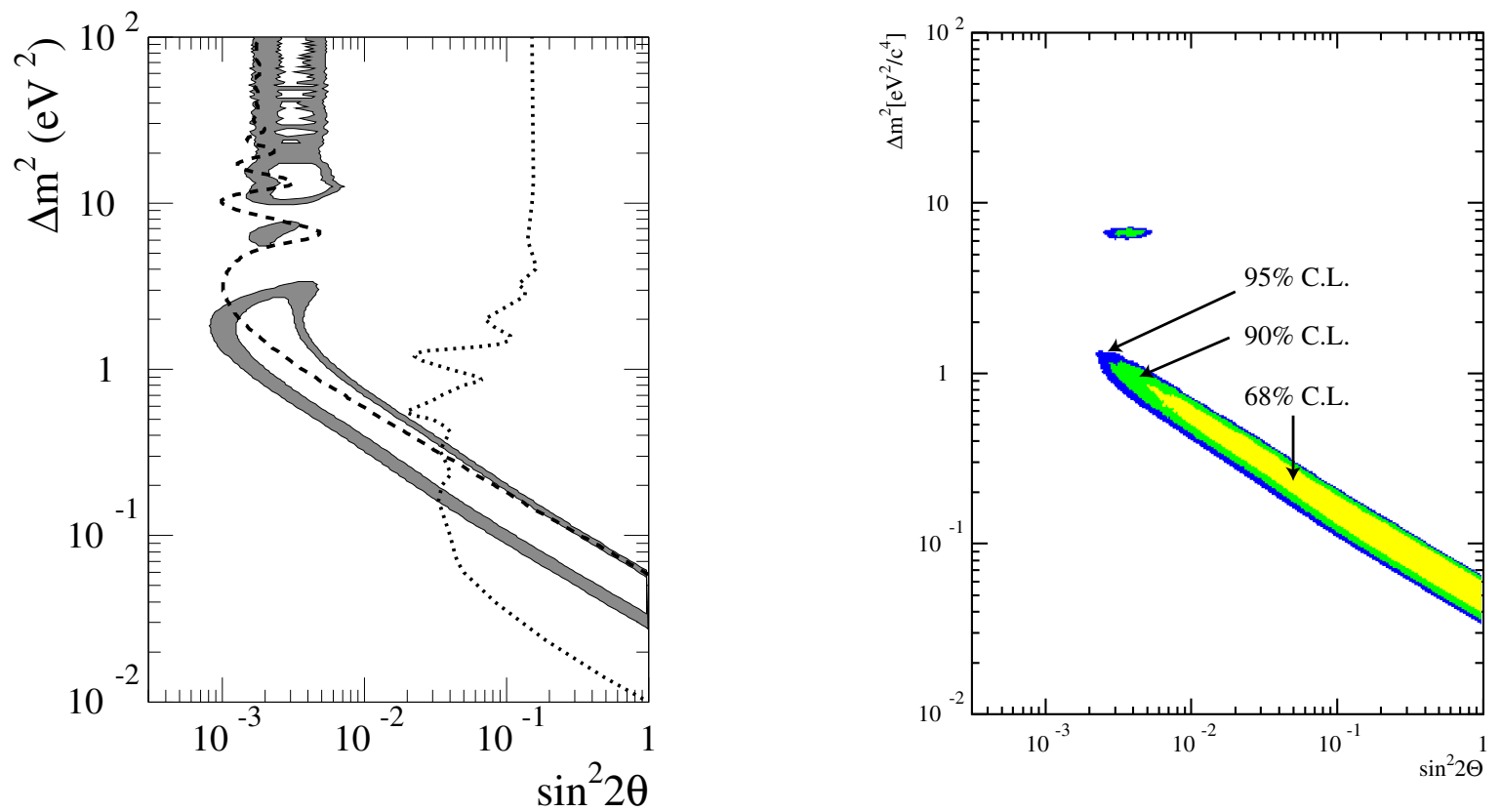

Figure 1.5: Left: the filled area shows the LSND 90\% and 99\% CL allowed region in oscillation parameter space [37]; the dashed and dotted curves show the 90\% CL upper limits on $\bar{\nu}_{\mu} \rightarrow \bar{\nu}_{e}$ oscillations from KARMEN [49], and $\bar{\nu}_{e} \rightarrow \bar{\nu}_{\not}$ oscillations from Bugey [51], respectively. Right: joint LSND-KARMEN allowed region in oscillation parameter space, assuming statistical compatibility among the two experimental outcomes [56].

\subsection{Other Neutrino Mass and Mixing Measure- ments}

Neutrino oscillations experiments are not the only way to probe neutrino masses and mixings. We briefly discuss here three more examples: tritium $\beta$ decay kinematics measurements, neutrinoless double $\beta$ decay searches, and observational cosmology.

\subsubsection{Laboratory-Based Neutrino Mass Searches}

Neutrino oscillation experiments cannot measure the absolute scale of neutrino masses. On the other hand, the differential electron energy spectrum in $\beta$ decay experiments is affected by both neutrino masses, and by the mixings defining the electron neutrino 
state in terms of mass eigenstates. The region of interest to the study of neutrino properties is located near the $\beta$ endpoint. The most sensitive searches conducted so far are based upon the decay of tritium, via ${ }^{3} \mathrm{H} \rightarrow{ }^{3} \mathrm{He}^{+} e^{-} \bar{\nu}_{e}$, mostly because of the very low $\beta$ endpoint energy of this element $(18.6 \mathrm{keV})$. For a spectrometer integrating over the electron energy interval $\delta$ near the $\beta$-decay endpoint, the count rate is [57]:

$$
n(\delta)=\frac{\bar{R}}{3} \sum_{i=1}^{n}\left|U_{e i}\right|^{2}\left(\delta^{2}-m_{i}^{2}\right)^{3 / 2}
$$

where the quantity $\bar{R}$ does not depend on the small neutrino masses and mixings, and and only the neutrino masses $m_{i}$ such that $\delta>m_{i}$ are considered in the summation. From the experimental point of view, tritium $\beta$ decay results are generally expressed in terms of a single effective mass $m\left(\nu_{e}\right)$ :

$$
n(\delta)=\frac{\bar{R}}{3}\left(\delta^{2}-m\left(\nu_{e}\right)^{2}\right)^{3 / 2}
$$

where $m\left(\nu_{e}\right)$ is the fit mass parameter. In the limit $\delta^{2} \gg m_{i}^{2}$, the relation between the true masses and mixings to the fitted mass $m\left(\nu_{e}\right)$ is independent from the integration interval $\delta$ :

$$
m\left(\nu_{e}\right)^{2} \simeq \sum_{i=1}^{n}\left|U_{e i}\right|^{2} m_{i}^{2}
$$

The current best measurements on $m\left(\nu_{e}\right)^{2}$ come from the Troitsk and Mainz experiments [58], which have very similar $m\left(\nu_{e}\right)^{2}$ sensitivities. Both found no evidence for a nonzero $m\left(\nu_{e}\right)^{2}$ value; the latest Mainz result is $m\left(\nu_{e}\right)^{2}=-1.6 \pm 2.5 \pm 2.1 \mathrm{eV}^{2}$, or $m\left(\nu_{e}\right) \leq 2.2 \mathrm{eV}$ at $95 \% \mathrm{CL}$, using $\delta=70 \mathrm{eV}$ [58]. The planned tritium $\beta$ decay experiment KATRIN should be able to improve the sensitivity to $m\left(\nu_{e}\right)$ by roughly an order of magnitude in the forthcoming years, thanks to its better statistics, energy resolution, and background rejection [59].

Neutrino oscillations do not probe the neutrino Dirac/Majorana character. On the other hand, the neutrinoless double-beta $(0 \nu \beta \beta)$ decay process is possible only if neutrinos are Majorana particles, while it is forbidden for Dirac neutrinos. Moreover, assuming that neutrinos are Majorana particles, $0 \nu \beta \beta$ searches probe also neutrino 
masses and mixings. Double-beta decay is a rare nuclear transition accompanied by the simultaneous emission of two electrons. The dominant mode is the second order weak process $(\mathrm{A}, \mathrm{Z}) \rightarrow(\mathrm{A}, \mathrm{Z}+2) e^{-} e^{-} \bar{\nu}_{e} \bar{\nu}_{e}$, or $2 \nu \beta \beta$, which conserves lepton number and is therefore allowed within the Standard Model. Neutrinoless double-beta decay proceeds without antineutrino emission, via $(\mathrm{A}, \mathrm{Z}) \rightarrow(\mathrm{A}, \mathrm{Z}+2) e^{-} e^{-}$. The experimental signature of $0 \nu \beta \beta$ is given by a measurement for the sum of the two electron energies consistent with the $Q$-value of the transition. The $0 \nu \beta \beta$ rate $\left(T_{1 / 2}^{0 \nu}\right)^{-1}$ is related to the effective neutrino Majorana mass $m_{\beta \beta}$ via [60]:

$$
\left(T_{1 / 2}^{0 \nu}\right)^{-1}=G^{0 \nu}\left|M^{0 \nu}\right|^{2} m_{\beta \beta}^{2}
$$

where $G^{0 \nu}$ is a phase space factor, $M^{0 \nu}$ is a nuclear matrix element, and the effective Majorana mass is given by:

$$
m_{\beta \beta}=\left|\sum_{i} U_{e i}^{2} m_{i}\right|
$$

where the $U_{e i}$ mixing matrix elements are in general complex, and the resulting expression for $m_{\beta \beta}$ may in general depend on both Dirac and Majorana phases. No convincing indication of $0 \nu \beta \beta$ has been found, so far, and an upper limit $m_{\beta \beta} \lesssim 1 \mathrm{eV}$ on the effective neutrino Majorana mass has been obtained [60]. The uncertainties in the nuclear matrix elements evaluation result in about a factor of 3 uncertainty in the Majorana mass uncertainty, for a given $0 \nu \beta \beta$ rate. Future experiments with significantly improved sensitivities are being planned [48].

\subsubsection{Neutrinos and Cosmology}

Observational cosmology can also constrain neutrino properties. Two examples are discussed here: the neutrino energy density during the Big Bang Nucleosynthesis $(\mathrm{BBN})$ era affects the primordial abundances of Helium and other elements, and neutrino masses affect the large scale structure formation of the Universe. 
The Helium primordial abundance is set by the neutron-to-proton ratio at weak freeze-out, that is at the epoch (temperature) at which the rate for the weak interaction process $p e^{-} \leftrightarrow n \nu_{e}$ equals the expansion rate of the Universe. In general, the higher the neutrino energy density, or equivalently the so-called number of effective neutrino species $N_{\text {eff }}$ present during BBN, the higher the expansion rate, and the higher the freeze-out temperature $T_{f}$ is. In the absence of a lepton asymmetry, generally expressed in terms of non-zero neutrino chemical potentials, the neutronto-proton ratio is related to $T_{f}$ via $n / p \simeq \exp \left[-\left(m_{n}-m_{p}\right) / T_{f}\right]$, where $m_{n}$ and $m_{p}$ are the neutron and proton masses, respectively. Therefore, the higher $N_{\text {eff }}$, the higher the neutron-to-proton ratio, and the higher the primordial Helium abundance is. The measured primordial Helium abundance, in conjunction with standard cosmology assumptions, yields $1.7<N_{\text {eff }}<3.5$ at $95 \%$ confidence level [61].

Light, active neutrinos decouple from the Universe thermal bath when they are relativistic. In this case, the free-streaming scale of neutrinos is therefore of the order of the Universe horizon at that epoch. Over distances smaller than the neutrino free streaming scale, massive neutrinos tend to wash out matter clustering due to gravity. As the Universe expands, massive neutrinos become eventually non-relativistic, their free-streaming scale does not scale as the event horizon anymore, and the effect of neutrinos on structure formation becomes negligible. The observations of the cosmic microwave background anisotropies, and of the power spectrum of large-scale matter structure, are consistent with a negligible effect from non-zero neutrino masses, and typical upper limits on the sum of neutrino masses of $\sum_{i} m_{i} \lesssim 1 \mathrm{eV}$ are obtained 62 .

In particular, cosmology may provide constraints on sterile neutrinos. Predictions of standard cosmology assume that light sterile neutrino species, if they exist, are present in the early Universe in the same abundances as the active species. In this picture, massive sterile neutrinos with significant mixing to active neutrinos are expected to alter both the Helium abundance and the matter power spectrum pre- 
dictions, in disagreement with observations. However, several mechanisms have been proposed that would suppress the sterile neutrino abundances in cosmology: primordial lepton asymmetries [63, 66, additional neutrino interactions [65], low reheating temperature [64], or other possibilities [61]. We briefly describe the first two, very general, mechanisms.

We first consider the possibility that a relic neutrino asymmetry is present in the early Universe, that is $L_{\nu_{\alpha}} \equiv\left(n_{\nu_{\alpha}}-n_{\bar{\nu}_{\alpha}}\right) / n_{\gamma} \neq 0$, where $n_{\nu_{\alpha}}$ and $n_{\bar{\nu}_{\alpha}}$ are neutrino and antineutrino number densities for the neutrino flavor $\alpha$, and $n_{\gamma}$ is the photon number density. In particular, we consider the effect that a non-zero $L_{\nu_{\alpha}}$ value would have in governing the $\nu_{\alpha} \leftrightarrow \nu_{s}$ conversion rate via oscillations; we assume here (as it is customary) that neutrino oscillations are the main mechanism to populate the early Universe with sterile neutrinos that are in thermal equilibrium with the other relativistic degrees of freedom present. In the case of a net relic neutrino asymmetry, the cumulative effect of neutrino-neutrino, coherent forward scattering interactions can be described by an effective, non-zero, matter potential. If the neutrino masses and mixings describing active-sterile neutrino oscillations are such that resonant, matterinduced $\nu_{\alpha} \leftrightarrow \nu_{s}$ oscillations would only happen after neutrino decoupling $\left(T_{\mathrm{dec}} \simeq 3\right.$ $\mathrm{MeV})$, at temperatures $T_{\text {res }}<T_{\text {dec }}$, the presence of this additional lepton number matter potential would drive the oscillations further off-resonance, suppressing the effective active-sterile neutrino mixing, and therefore sterile neutrino production. It is shown that a net relic neutrino asymmetry $L_{\nu_{\alpha}} \gtrsim 5 \cdot 10^{-3}$ in any of the active neutrino flavors is sufficient to essentially fully suppress sterile neutrino production in the early Universe [66]. This condition is consistent with present upper bounds on relic neutrino asymmetries, given by $L_{\nu_{\alpha}} \lesssim 0.1[67$.

Another way to suppress neutrino production in the Universe is via additional neutrino interactions. For example, the coupling of neutrinos with a light boson $\phi$ (with $m_{\phi} \ll m_{\nu}$ ) has been considered [65]. In this scenario, neutrinos may remain in thermal equilibrium until late times, for example via $\nu \nu \leftrightarrow \phi \phi$, and neutrino decou- 
pling would occur when they are non-relativistic, that is when the process $\phi \phi \rightarrow \nu \nu$ would be kinematically forbidden. For sufficiently large couplings, neutrino freezeout would occur at temperatures $T_{\text {dec }} \ll m_{\nu}$, and their final abundance would be suppressed by a factor $\exp \left[-m_{\nu} / T_{\text {dec }}\right] \ll 1$, generating a neutrinoless Universe. The existence of such a light boson, with sufficiently large couplings to neutrinos for this mechanism to occur, currently evades all cosmological limits, and it is therefore allowed.

\subsection{The MiniBooNE $\nu_{\mu} \rightarrow \nu_{e}$ Search}

The main goal of the MiniBooNE experiment is to unambiguously confirm or refute the evidence for $\bar{\nu}_{\mu} \rightarrow \bar{\nu}_{e}$ oscillations seen by the LSND experiment at Los Alamos. This is important because, as stated above, the LSND oscillation result is incompatible with the robust evidence for solar and atmospheric neutrino oscillations in the simplest three-neutrino mixing paradigm.

The MiniBooNE experiment is probing the oscillation parameter space indicated by LSND via a $\nu_{\mu} \rightarrow \nu_{e}$ search, and possibly by a future $\bar{\nu}_{\mu} \rightarrow \bar{\nu}_{e}$ search. The large sample of neutrino interactions detected at MiniBooNE will allow coverage of the full LSND allowed region at $4 \sigma$ significance, as shown in the left panel of Fig. 1.6 [68]. Furthermore, the sources of systematic uncertainties affecting the MiniBooNE $\nu_{\mu} \rightarrow \nu_{e}$ search are very different than the ones affecting the LSND result. This is because, despite the similar neutrino $L / E$ range measured in the two experiments, neutrinos detected at MiniBooNE are about a factor of 20 more energetic than LSND neutrinos, with typical energies of the order of $1 \mathrm{GeV}$. As a consequence, both the oscillation signal detection process, and the main backgrounds to the oscillation search, are different in MiniBooNE and LSND. The oscillation signal detection process used for the MiniBooNE $\nu_{\mu} \rightarrow \nu_{e}$ search is the electron neutrino, charged-current, quasielastic neutrino interaction, $\nu_{e} n \rightarrow e^{-} p$. The main backgrounds to the oscillation 
search are due to the intrinsic (irreducible) $\nu_{e}$ contamination in the beam from muon and kaon decays, and from $\nu_{\mu} N \rightarrow \nu_{\mu} N \pi^{0}$ neutrino neutral current interactions, where the two Cherenkov rings from $\pi^{0} \rightarrow \gamma \gamma$ decays are misidentified as a single, electron-like Cherenkov ring.

In the case of a confirmation of the LSND signal, MiniBooNE may also be able to discern the mass and mixing parameters responsible for neutrino oscillations, as shown in the right panel of Fig. 1.6 68. A rough determination of the LSND neutrino mass and mixing parameters would not only be of extraordinary interest in itself, but would also serve as guidance for the planning of future short-baseline experiments such as a two-detector experiment at Fermilab, BooNE.

\subsection{Synopsis of this Thesis}

This thesis is organized as follows. Chapter 2 presents my work on the phenomenology of sterile neutrinos. Chapter 3 describes the MiniBooNE neutrino beamline and its magnetic focusing horn, which I had primary responsibility within the collaboration for understanding and monitoring. Chapter 4 discusses in detail the neutrino flux predictions for the MiniBooNE experiment; the beam Monte Carlo simulation developed for this purpose has been my main software responsibility. Chapters 5 and 6 summarize neutrino interactions in the $1 \mathrm{GeV}$ energy regime, and the MiniBooNE detector, respectively. Chapters 7 and 8 present the physics analysis undertaken, that is the characterization of charged-current, quasi-elastic $\left(\nu_{\mu} n \rightarrow \mu^{-} p\right)$ interactions, and the study of muon neutrino disappearance via muon-to-sterile neutrino oscillations. 

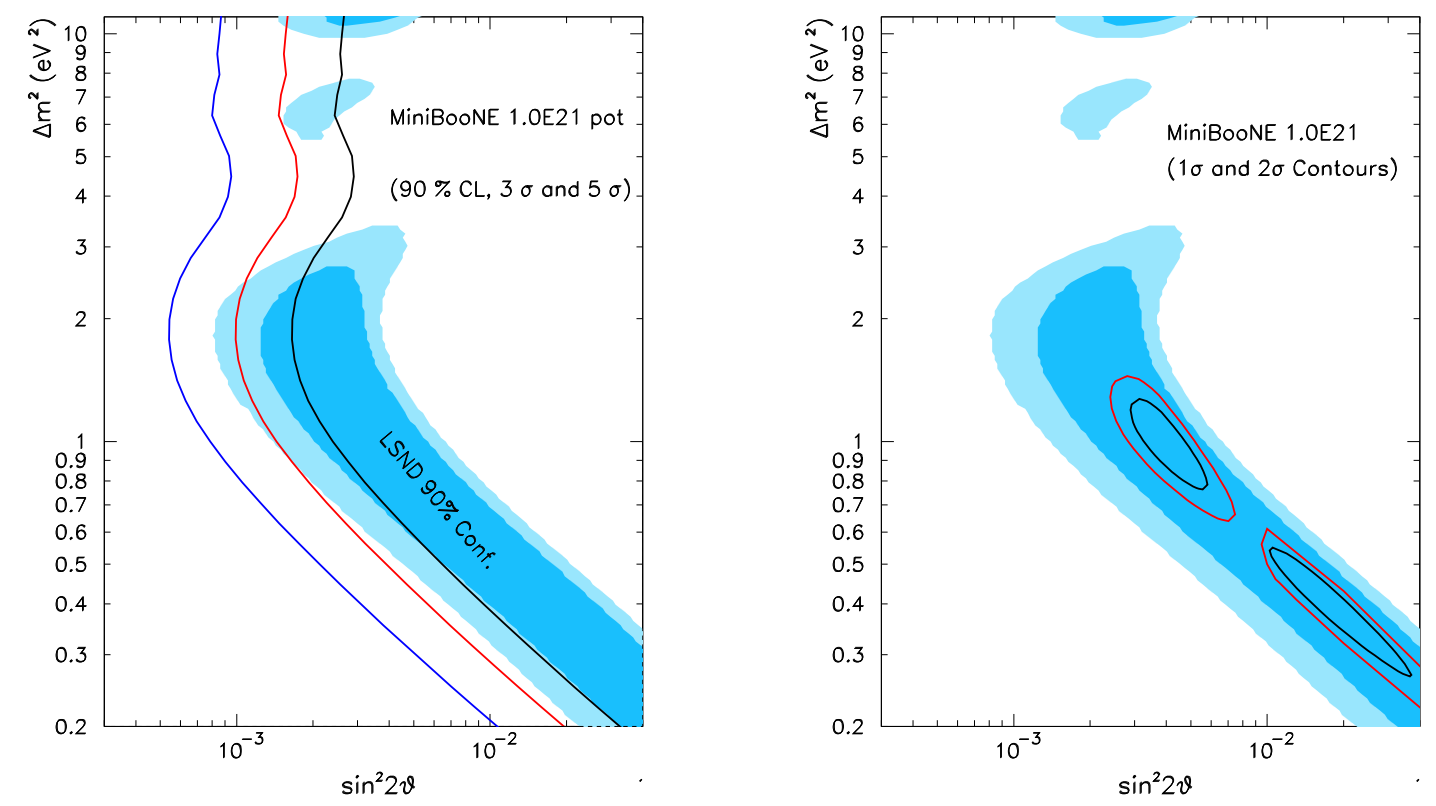

Figure 1.6: Left: MiniBooNE oscillation sensitivity for $10^{21}$ protons on target. The dark (light) blue areas are the LSND 90\% (99\%) CL allowed regions. The three curves give the 90\%, $3 \sigma$, and $5 \sigma$ sensitivity regions for MiniBooNE. Right: one and two $\sigma$ contours for an oscillation signal with $\Delta m^{2}=0.4$ or $1.0 \mathrm{eV}^{2}$, and for a data sample corresponding to $10^{21}$ protons on target [68]. 


\section{Chapter 2}

\section{Phenomenology of Sterile}

\section{Neutrinos}

In this Chapter, the phenomenological work on sterile neutrinos done during the course of this thesis is presented. The Chapter is divided into three sections:

- Section 2.1 discusses the constraints on sterile neutrino mass hierarchies, obtained from the observation of supernova neutrinos;

- Section 2.2 discusses the constraints on the number of light sterile neutrinos, their masses and their mixings, achieved from a combined analysis of accelerator-based and reactor-based short-baseline neutrino experiments;

- Section 2.3 discusses the constraints on leptonic CP-violation in $(3+2)$ sterile neutrino models obtained from short-baseline neutrino experiments, and implications for the interpretation of the forthcoming MiniBooNE results on $\nu_{\mu} \rightarrow \nu_{e}$ and (possibly) $\bar{\nu}_{\mu} \rightarrow \bar{\nu}_{e}$ oscillations. 


\subsection{Supernova Neutrino Experiments and the Neu- trino Mass Hierarchy}

The very dense matter environment traversed by supernova neutrinos affects how neutrinos are believed to oscillate among flavors: in this case, the neutrino mass hierarchy, that is the ordering of the neutrino mass eigenvalues, plays a role. Under certain approximations, it is possible to analytically derive the probability for muon and tau neutrinos produced in supernova explosions to be detected as electron neutrinos on Earth, as a function of the neutrino mass hierarchy and mixing parameters.

Detailed simulation models of the neutrino transport in core-collapse supernova explosions indicate different energy distributions for the electron, muon, and tau neutrino flavors at their surfaces of last scattering.

Electron neutrinos originated from the SN1987A supernova explosion that occurred in the neighborhood of our galaxy have been observed on Earth. Therefore, the observation of the electron neutrino energy spectrum on Earth provides (modeldependent) constraints on muon/tau neutrino transitions to electron neutrinos, and therefore on the neutrino mass hierarchy and mixings.

The following paper (see Ref. [69] and references therein), written in collaboration with J. Conrad, discusses these constraints for various sterile neutrino models. The main result of the paper is that several sterile neutrino mass hierarchies are strongly disfavored, based on the SN1987A observations, providing an experimental way to constrain the large "phase space" of sterile neutrino models that are present in the literature. 


\title{
Supernova neutrinos and the LSND evidence for neutrino oscillations
}

\author{
Michel Sorel* and Janet Conrad ${ }^{\dagger}$ \\ Department of Physics, Columbia University, New York, New York 10027 \\ (Received 15 December 2001; published 23 August 2002)
}

\begin{abstract}
The observation of the $\bar{\nu}_{e}$ energy spectrum from a supernova burst can provide constraints on neutrino oscillations. We derive formulas for adiabatic oscillations of supernova antineutrinos for a variety of 3- and 4-neutrino mixing schemes and mass hierarchies which are consistent with the Liquid Scintillation Neutrino Detector (LSND) evidence for $\bar{\nu}_{\mu} \rightarrow \bar{\nu}_{e}$ oscillations. Finally, we explore the constraints on these models and LSND given by the supernova SN 1987A $\bar{\nu}_{e}$ 's observed by the Kamiokande-2 and IMB-3 detectors.
\end{abstract}

DOI: 10.1103/PhysRevD.66.033009

PACS number(s): 14.60.Pq, 14.60.St, 26.50.+x, 97.60.Bw

\section{INTRODUCTION}

In recent years, the treatment of neutrino transport in the environment of a core-collapse supernova (SN) explosion has improved to the point of making realistic predictions on the observables for neutrinos reaching the Earth [1-4]. Of particular interest for this paper are the average energies at the neutrinospheres, i.e. the surfaces of last scattering for the neutrinos, estimated to be $10-13 \mathrm{MeV}$ for $\nu_{e}, 14-17 \mathrm{MeV}$ for $\bar{\nu}_{e}, 23-27 \mathrm{MeV}$ for $\nu_{\mu, \tau}, \bar{\nu}_{\mu, \tau}[2,4]$.

The differences in temperatures between the various neutrino flavors can be qualitatively understood. Heavy-lepton neutrinos can interact only via neutral current (NC) processes, the main contribution to their transport opacity coming from neutrino-nucleon scattering, which dominates over neutrino-electron scattering. In addition to this same $\mathrm{NC}$ contribution, the transport opacity for $\nu_{e}$ 's and $\bar{\nu}_{e}$ 's depends also on the charged current (CC) absorptions $\nu_{e}+n \rightarrow p+e^{-}$and $\bar{\nu}_{e}+p \rightarrow n+e^{+}$, respectively. Therefore, the $\nu_{e^{-}}$and $\bar{\nu}_{e}$-spheres are located at larger radii with respect to the other neutrinospheres, that is at lower densities and lower temperatures. Moreover, in a neutron-rich environment, $\nu_{e}+n \rightarrow p$ $+e^{-}$dominates over $\bar{\nu}_{e}+p \rightarrow n+e^{+}$: the emergent $\nu_{e}$ 's originate from layers farther outside the center of the star compared to $\bar{\nu}_{e}$ 's, therefore at lower temperatures. The total energy released in a $\mathrm{SN}$ explosion is approximately equipartitioned between the different neutrino and antineutrino flavors [3].

The above predictions can be confronted with the observation of the supernova $\bar{\nu}_{e}$ energy spectrum detected on Earth. Neutrino oscillations are expected to modify the spectrum since $\left\langle E_{\bar{\nu}_{e}}\right\rangle\left\langle\left\langle E_{\bar{\nu}_{\mu}, \bar{\nu}_{\tau}}\right\rangle\right.$. The energy dependence of the neutrino cross section in the detector material, approximately $\sigma_{\nu_{e} p}^{-} \propto\left(E_{\nu_{e}}-1.29 \mathrm{MeV}\right)^{2}[5]$, helps in making the $\bar{\nu}_{e}$ energy spectrum distortion a sensitive experimental probe to neutrino oscillations. This is because higher energy neutrinos interact significantly more than lower energy ones.

\footnotetext{
*Electronic address: sorel@fnal.gov

†Electronic address: conrad@fnal.gov
}

We show that the extent of the spectrum modification depends crucially on the specifics of the neutrino mixing scheme and on the neutrino mass hierarchy under consideration, and we derive the relevant formulas assuming an adiabatic propagation for the antineutrinos in the supernova environment. Antineutrinos propagate adiabatically if the varying matter density they encounter changes slowly enough so that transitions between local (instantaneous) Hamiltonian eigenstates can be neglected throughout the entire antineutrino propagation. So far, neutrinos from one supernova have been detected and their energy measured: SN 1987A was observed by the Kamiokande-2 and IMB-3 detectors. The overall 20 events seen by those two detectors have all been interpreted as $\bar{\nu}_{e}$ interactions [6]. We examine the constraint of such observations on the LSND allowed region of $\bar{\nu}_{\mu} \rightarrow \bar{\nu}_{e}$ oscillations [7], for various neutrino mass and mixing models. If the LSND evidence is confirmed by the MiniBooNE experiment [8], several models can be excluded or constrained on the basis of the observations of the supernova SN 1987A and possibly future supernovæ.

\section{ADIABATIC OSCILLATIONS AND NEUTRINO MIXING SCHEMES}

\section{A. $\bar{\nu}_{e}$ energy spectrum and the permutation factor}

In the presence of neutrino oscillations, the $\bar{\nu}_{e}$ flux reaching the Earth, $F_{\nu_{e}}$, can be different from the primary flux at the neutrinosphere, $F_{\nu_{e}}^{0}$. We will assume that, at production, the energy of active antineutrinos is equally divided into the three active flavors, i.e. that $\int_{0}^{\infty} d E_{\bar{\nu}_{\alpha}} E_{\bar{\nu}_{\alpha}} F_{\bar{\nu}_{\alpha}}^{0}$ has the same numerical value for $\alpha=e, \mu, \tau$. Moreover, we will also consider neutrino mixing models where the three active neutrino species are augmented by a fourth sterile neutrino with no standard weak couplings: in those cases, we will assume that the sterile component is negligible at production.

The neutrino flux reaching the Earth is

$$
\begin{aligned}
F_{\nu_{e}}^{-} & =\left(p_{\mu \rightarrow e}+p_{\tau \rightarrow e}\right) F_{\bar{\nu}_{\mu}}^{0}+p_{e \rightarrow e} F_{\bar{\nu}_{e}}^{0} \\
& \propto\left[p F_{\bar{\nu}_{\mu}}^{0}+(1-p) F_{\bar{\nu}_{e}}^{0}\right]
\end{aligned}
$$

where we have defined the permutation factor $p$ as 


$$
p=\frac{p_{\mu \rightarrow e}+p_{\tau \rightarrow e}}{p_{\mu \rightarrow e}+p_{\tau \rightarrow e}+p_{e \rightarrow e}}
$$

and $p_{\mu, \tau, e \rightarrow e}$ are the probabilities for a $\bar{\nu}_{\mu}, \bar{\nu}_{\tau}, \bar{\nu}_{e}$ respectively at the neutrinosphere to oscillate into a $\bar{\nu}_{e}$. In Eqs. (1), (2), we have assumed that $p$ is energy-independent (as will be justified later), and that $\left\langle E_{\bar{\nu}_{\mu}}\right\rangle=\left\langle E_{\bar{\nu}_{\tau}}\right\rangle$. In Eq. (1), we neglect the (energy-independent) proportionality factor since we will not deal with event rates, but only with neutrino energy distributions.

\section{B. Neutrino propagation in the adiabatic approximation}

In vacuum, the Hamiltonian that governs neutrino propagation is diagonal in the mass eigenstate basis $\left|\nu_{i}\right\rangle$ :

$$
\left(H_{0}\right)_{i j} \equiv\left\langle\nu_{i}\left|H_{0}\right| \nu_{j}\right\rangle=E_{i} \delta_{i j} .
$$

If the neutrinos all have the same relativistic momentum $p$, their energies $E_{i}$ differ only by a term proportional to their squared-mass differences, since $E_{i} \simeq p+m_{i}^{2} / 2 p$. If $U$ is the unitary mixing matrix that relates the flavor eigenstates $\left|\nu_{\alpha}\right\rangle$ to the mass eigenstates via $\left|\nu_{\alpha}\right\rangle=U_{\alpha i}\left|\nu_{i}\right\rangle$, the elements of the vacuum Hamiltonian in the flavor basis are given by [9]:

$$
\left(H_{0}\right)_{\alpha \beta}=U_{\alpha i}^{*} U_{\beta i} \frac{m_{i}^{2}}{2 p}
$$

where we have neglected the contribution $p \delta_{\alpha \beta}$ in $\left(H_{0}\right)_{\alpha \beta}$, which is irrelevant for neutrino oscillations.

In matter, $\bar{\nu}_{e}$ 's undergo coherent charged current (CC) forward-scattering from electrons, and all active flavor antineutrinos coherent neutral current (NC) forward-scattering from electrons, protons, and neutrons in the medium. These processes give rise to an interaction potential $V=V_{W}+V_{Z}$, which is diagonal in the flavor basis and proportional to the matter density $\rho$ :

$$
(V)_{\alpha \beta}=A_{\alpha} \frac{G_{F} \rho}{m_{N}} \delta_{\alpha \beta}
$$

where $A_{\alpha}$ is a proportionality constant, in general different for $\alpha=e, \mu, \tau$, or $s, G_{F}$ the Fermi constant, and $m_{N}$ the nucleon mass. The relevant Hamiltonian for neutrino propagation in matter is therefore $H \equiv H_{0}+V$.

At the neutrinosphere, the density $\rho$ is so high $\left(\sim 10^{12} \mathrm{~g} / \mathrm{cm}^{3}[1]\right)$ that the interaction potential dominates over the vacuum Hamiltonian, so that the propagation eigenstates coincide with the flavor eigenstates. As the propagation eigenstates free-stream outwards, toward regions of lower density, their flavor composition changes, ultimately reaching the flavor composition of the mass eigenstates in the vacuum. Given that the neutrinos escape the SN as mass eigenstates, no further flavor oscillations occur on their path to the Earth.

More specifically, making use of the adiabatic approximation and of the fact that no energy-level crossing is permitted, the flavor eigenstate at the neutrinosphere with the maxi-

\begin{tabular}{|c|c|c|}
\hline Model & Hierarchy & Propagation \\
\hline Normal $(1+1+1)$ & $m_{3}>m_{2}>m_{1}$ & $\begin{array}{l}\bar{\nu}_{\gamma} \rightarrow \bar{\nu}_{3} \\
\bar{\nu}_{\beta} \rightarrow \bar{\nu}_{2} \\
\bar{\nu}_{\alpha} \rightarrow \bar{\nu}_{1}\end{array}$ \\
\hline Normal $(1+1)$ & $m_{2} \gg m_{1}$ & $\begin{array}{l}\bar{\nu}_{\mu} \rightarrow \bar{\nu}_{2} \\
\bar{\nu}_{e} \rightarrow \bar{\nu}_{1}\end{array}$ \\
\hline LSND-inverted $(1+1)$ & $m_{1} \gg m_{2}$ & $\begin{array}{l}\bar{\nu}_{\mu} \rightarrow \bar{\nu}_{1} \\
\bar{\nu}_{e} \rightarrow \bar{\nu}_{2}\end{array}$ \\
\hline Normal $(2+1)$ & $m_{3}>m_{2} \gg m_{1}$ & $\begin{array}{l}\bar{\nu}_{\mu} \rightarrow \bar{\nu}_{3} \\
\bar{\nu}_{\tau} \rightarrow \bar{\nu}_{2} \\
\bar{\nu}_{e} \rightarrow \bar{\nu}_{1}\end{array}$ \\
\hline LSND-inverted $(2+1)$ & $m_{1} \gg m_{3}>m_{2}$ & $\begin{array}{l}\bar{\nu}_{\mu} \rightarrow \bar{\nu}_{1} \\
\bar{\nu}_{\tau} \rightarrow \bar{\nu}_{3} \\
\bar{\nu}_{e} \rightarrow \bar{\nu}_{2}\end{array}$ \\
\hline Normal $(2+2)$ & $m_{3}>m_{2} \gg m_{1}>m_{0}$ & $\begin{array}{l}\bar{\nu}_{\mu} \rightarrow \bar{\nu}_{3} \\
\bar{\nu}_{\tau} \rightarrow \bar{\nu}_{2} \\
\bar{\nu}_{s} \rightarrow \bar{\nu}_{1} \\
\bar{\nu}_{e} \rightarrow \bar{\nu}_{0}\end{array}$ \\
\hline LSND-inverted $(2+2)$ & $m_{1}>m_{0} \gg m_{3}>m_{2}$ & $\begin{array}{l}\bar{\nu}_{\mu} \rightarrow \bar{\nu}_{1} \\
\bar{\nu}_{\tau} \rightarrow \bar{\nu}_{0} \\
\bar{\nu}_{s} \rightarrow \bar{\nu}_{3} \\
\bar{\nu}_{e} \rightarrow \bar{\nu}_{2}\end{array}$ \\
\hline Normal $(3+1)$ & $m_{4} \gg m_{3}>m_{2}>m_{1}$ & $\begin{array}{l}\bar{\nu}_{\mu} \rightarrow \bar{\nu}_{4} \\
\bar{\nu}_{\tau} \rightarrow \bar{\nu}_{3} \\
\bar{\nu}_{s} \rightarrow \bar{\nu}_{2} \\
\bar{\nu}_{e} \rightarrow \bar{\nu}_{1}\end{array}$ \\
\hline LSND-inverted $(3+1)$ & $m_{3}>m_{2}>m_{1} \gg m_{4}$ & $\begin{array}{l}\bar{\nu}_{\mu} \rightarrow \bar{\nu}_{3} \\
\bar{\nu}_{\tau} \rightarrow \bar{\nu}_{2} \\
\bar{\nu}_{s} \rightarrow \bar{\nu}_{1} \\
\bar{\nu}_{e} \rightarrow \bar{\nu}_{4}\end{array}$ \\
\hline
\end{tabular}
mum interaction potential reaches Earth as the mass
TABLE I. Adiabatic neutrino propagation in the SN ejecta for the neutrino mixing models considered.

eigenstate with the biggest neutrino mass. In general, the energy level order is maintained throughout the neutrino propagation in the SN ejecta. This is illustrated in Table I for three neutrinos in the row labeled "normal $(1+1+1)$," where we have taken $A_{\gamma}>A_{\beta}>A_{\alpha}$ and $m_{3}>m_{2}>m_{1}$.

For example, the probability for a $\bar{\nu}_{\alpha}$ to emerge from the $\mathrm{SN}$ environment as a $\bar{\nu}_{\beta}$ is given by

$$
\begin{aligned}
p_{\alpha \rightarrow \beta} & =\left|\left\langle\bar{\nu}_{\beta}\left|U^{\text {evol }}\right| \bar{\nu}_{\alpha}\right\rangle\right|^{2}=\left|\left\langle U_{\beta i} \bar{\nu}_{i}\left|U^{e v o l}\right| \bar{\nu}_{\alpha}\right\rangle\right|^{2} \\
& =\left|U_{\beta i}^{*} \delta_{i, 1}\right|^{2}=\left|U_{\beta 1}\right|^{2}
\end{aligned}
$$

where $U^{\text {evol }}$ is the adiabatic evolution operator. In Eq. (6), we have used Table I to get

$$
\left\langle\bar{\nu}_{i}\left|U^{e v o l}\right| \bar{\nu}_{\alpha}\right\rangle=\delta_{i, 1} .
$$


TABLE II. Results on the probabilities $p_{\mu, \tau, e \rightarrow e}$ for a $\bar{\nu}_{\mu, \tau, e}$ to emerge from the SN as a $\bar{\nu}_{e}$, the permutation factor $p$ of Eq. 2, and the LSND oscillation amplitude $\sin ^{2} 2 \vartheta_{L S N D}$, for the various neutrino mixing schemes considered.

\begin{tabular}{lcccccc}
\hline \hline Model & Mixing & $p_{\mu \rightarrow e}$ & $p_{\tau \rightarrow e}$ & $p_{e \rightarrow e}$ & $p$ & $\sin ^{2} 2 \vartheta_{L S N D}$ \\
\hline Normal (1+1) & Eq. (10) & $\sin ^{2} \vartheta$ & 0 & $\cos ^{2} \vartheta$ & $\sin ^{2} \vartheta$ & $\sin ^{2} 2 \vartheta=4 p(1-p)$ \\
LSND-inverted (1+1) & Eq. (10) & $\cos ^{2} \vartheta$ & 0 & $\sin ^{2} \vartheta$ & $\cos ^{2} \vartheta$ & $\sin ^{2} 2 \vartheta=4 p(1-p)$ \\
Normal (2+1) & Eq. (11) & $\frac{3}{4} \alpha^{2}$ & $\frac{1}{4} \alpha^{2}$ & 1 & $\alpha^{2} /\left(1+\alpha^{2}\right)$ & $4 \alpha^{2}=4 p /(1-p)$ \\
LSND-inverted (2+1) & Eq. (11) & 1 & $\frac{3}{4} \alpha^{2}$ & $\frac{1}{4} \alpha^{2}$ & $\left(1+\frac{3}{4} \alpha^{2}\right) /\left(1+\alpha^{2}\right)$ & $4 \alpha^{2}=4(1-p) /\left(p-\frac{3}{4}\right)$ \\
Normal (2+2) & Eq. (14) & $\beta^{2}$ & $\beta^{2}$ & $\frac{1}{2}$ & $4 \beta^{2} /\left(1+4 \beta^{2}\right)$ & $8 \beta^{2}=2 p /(1-p)$ \\
LSND-inverted (2+2) & Eq. (14) & $\frac{1}{2}$ & $\frac{1}{2}$ & $\beta^{2}$ & $1 /\left(1+\beta^{2}\right)$ & $8 \beta^{2}=8(1-p) / p$ \\
Normal (3+1) & Eq. (15) & $\gamma^{2}$ & 0 & $\frac{1}{2}$ & $2 \gamma^{2} /\left(1+2 \gamma^{2}\right)$ & $4 \gamma^{2} \delta^{2}=2 \delta^{2} p /(1-p)$ \\
LSND-inverted (3+1) & Eq. (15) & 0 & $\frac{1}{2}$ & $\gamma^{2}$ & $1 /\left(1+2 \gamma^{2}\right)$ & $4 \gamma^{2} \delta^{2}=2 \delta^{2}(1-p) / p$ \\
\hline \hline
\end{tabular}

This result can be immediately generalized to any number of antineutrino generations. Also, as long as the adiabatic approximation is satisfied, the formula does not depend on the specific dynamics for the neutrino propagation, for example on the number and position in the SN environment of Mikheyev-Smirnov-Wolfenstein (MSW) resonances. We will comment more on the validity of the adiabatic approximation in the next section.

In this paper, we consider three or four flavor components, including a sterile one. At tree-level, the proportionality factors $A_{\alpha}$ in the interaction potential for neutral matter are $[9,10]$

$$
A=\left\{\begin{array}{lll}
\left(1-3 Y_{e}\right) / \sqrt{2} & \text { for } & \bar{\nu}_{e}, \\
\left(1-Y_{e}\right) / \sqrt{2} & \text { for } & \bar{\nu}_{\mu}, \bar{\nu}_{\tau}, \\
0 & \text { for } & \bar{\nu}_{s},
\end{array}\right.
$$

where $Y_{e}$ is the electron fraction per nucleon. Following the assumptions of $[10,11]$, we use $Y_{e} \simeq\left(1+\left\langle E_{\nu_{e}}^{-}\right\rangle /\left\langle E_{\nu_{e}}\right\rangle\right)^{-1}$ $>1 / 3$ at the neutrinosphere. Considering also one-loop electroweak radiative corrections, a difference in the $\bar{\nu}_{\mu}$ and $\bar{\nu}_{\tau}$ interaction potentials of magnitude $\left(A_{\mu}-A_{\tau}\right) / A_{\mu} \sim 10^{-4}$ appears due to the difference in the charged lepton masses $[12,13]$. At the neutrinosphere, this second-order effect in the interaction potential dominates over the vacuum Hamiltonian terms (as long as $\left|m_{i}^{2}-m_{j}^{2}\right|<10 \mathrm{eV}^{2}$ for all $i, j$ ), and removes the $\bar{\nu}_{\mu}-\bar{\nu}_{\tau}$ degeneracy. Therefore, for the antineutrino channel considered here, we take

$$
A_{\mu}>A_{\tau}>A_{s}>A_{e} .
$$

For the neutrino channel, one should substitute $A \rightarrow-A$ in Eq. (8), and the order in Eq. (9) would be inverted.

Therefore, given a specific neutrino mass and mixing model, the permutation factor can be easily evaluated in the adiabatic approximation, and its numerical value does not depend on the neutrino energy. We will comment on possible energy-dependent Earth matter effects in the next section. In practice, one proceeds backwards: given a certain measured value of $p$, it is possible to constrain possible models for neutrino oscillations. This approach is used for example in
[13] to constrain models explaining the solar and atmospheric neutrino data; in this paper, we focus on 3 and 4-neutrino models explaining the Liquid Scintillation Neutrino Detector (LSND) data.

\section{Possible mixing schemes}

The results for the $\bar{\nu}_{\mu}, \bar{\nu}_{\tau}, \bar{\nu}_{e} \rightarrow \bar{\nu}_{e}$ adiabatic oscillation probabilities, the permutation factor $p$, and the LSND oscillation amplitude $\sin ^{2} 2 \vartheta$ as a function of the mixing parameters and $p$ for the eight possible mass and mixing schemes considered below are given in Table II. The mass hierarchy and the adiabatic propagation of the neutrino eigenstates for these mixing schemes are depicted in Table I.

The simplest possible mixing scheme is a $(1+1)$ model explaining only $\bar{\nu}_{\mu} \rightarrow \bar{\nu}_{e}$ LSND oscillations in vacuum, and not the atmospheric or solar oscillations:

$$
\left(\begin{array}{l}
\bar{\nu}_{e} \\
\bar{\nu}_{\mu}
\end{array}\right)=\left(\begin{array}{rr}
\cos \vartheta & \sin \vartheta \\
-\sin \vartheta & \cos \vartheta
\end{array}\right)\left(\begin{array}{l}
\bar{\nu}_{1} \\
\bar{\nu}_{2}
\end{array}\right),
$$

where the mixing angle $\vartheta$ can assume any value in the range $0<\vartheta<\pi / 4$.

We consider a $(2+1)$ model motivated, for example, by $C P T$-violating scenarios (see, e.g. [14]), in which atmospheric and LSND oscillations in the antineutrino channel are obtained via the mixing [15]:

$$
\left(\begin{array}{l}
\bar{\nu}_{e} \\
\bar{\nu}_{\mu} \\
\bar{\nu}_{\tau}
\end{array}\right)=\left(\begin{array}{ccc}
1 & -\frac{1}{2} \alpha & -\frac{\sqrt{3}}{2} \alpha \\
\alpha & \frac{1}{2} & \frac{\sqrt{3}}{2} \\
0 & -\frac{\sqrt{3}}{2} & \frac{1}{2}
\end{array}\right)\left(\begin{array}{c}
\bar{\nu}_{1} \\
\bar{\nu}_{2} \\
\bar{\nu}_{3}
\end{array}\right) .
$$

The matrix in Eq. (11) is chosen to ensure large $\bar{\nu}_{\mu} \rightarrow \bar{\nu}_{\tau}$ mixing for atmospheric neutrinos $\left(\sin ^{2} 2 \vartheta_{\text {atm }}=3 / 4\right)$, while the LSND $\bar{\nu}_{\mu} \rightarrow \bar{\nu}_{e}$ mixing is fixed by the parameter $\alpha$ $\left(\sin ^{2} 2 \vartheta_{L S N D}=4 \alpha^{2}\right)$.

The most popular models which explain the solar, atmospheric and LSND signatures (and the null results obtained 
by other experiments) via neutrino oscillations invoke the existence of a sterile neutrino $\bar{\nu}_{s}$. One example of a $(2$ +2 ) model is the following, which is taken from [16]:

$$
\left(\begin{array}{l}
\bar{\nu}_{s} \\
\bar{\nu}_{e} \\
\bar{\nu}_{\mu} \\
\bar{\nu}_{\tau}
\end{array}\right)=\left(\begin{array}{cccc}
\frac{1}{\sqrt{2}} & \frac{1}{\sqrt{2}} & 0 & 0 \\
-\frac{1}{\sqrt{2}} & \frac{1}{\sqrt{2}} & \beta & \beta \\
\beta & -\beta & \frac{1}{\sqrt{2}} & \frac{1}{\sqrt{2}} \\
0 & 0 & -\frac{1}{\sqrt{2}} & \frac{1}{\sqrt{2}}
\end{array}\right)\left(\begin{array}{c}
\bar{\nu}_{0} \\
\bar{\nu}_{1} \\
\bar{\nu}_{2} \\
\bar{\nu}_{3}
\end{array}\right)
$$

where one pair of nearly degenerate mass eigenstates has maximal $\nu_{e} \rightarrow \nu_{s}$ mixing for solar neutrinos and the other pair has maximal $\nu_{\mu} \rightarrow \nu_{\tau}$ mixing for atmospheric neutrinos. Small inter-doublet mixings through the $\beta$ parameter accommodate the LSND result $\left(\sin ^{2} \vartheta_{L S N D}=8 \beta^{2}\right)$.

Recent experimental results [17] show that pure $\nu_{e} \rightarrow \nu_{s}$ solar oscillations are excluded at high significance. We therefore consider a more general $(2+2)$ scenario, in which solar neutrinos can undergo any combination of $\nu_{e} \rightarrow \nu_{s}$ and $\nu_{e}$ $\rightarrow \nu_{\tau}$ oscillations, while atmospheric neutrinos can undergo any combination of $\nu_{\mu} \rightarrow \nu_{\tau}$ and $\nu_{\mu} \rightarrow \nu_{s}$ oscillations. We follow the procedure in [18] to obtain this more general mixing starting from Eq. (12), by substituting the $\left(\bar{\nu}_{s}, \bar{\nu}_{\tau}\right)$ states with the rotated states $\left(\bar{\nu}_{s}^{\prime}, \bar{\nu}_{\tau}^{\prime}\right)$ :

$$
\left(\begin{array}{c}
\bar{\nu}_{s}^{\prime} \\
\bar{\nu}_{\tau}^{\prime}
\end{array}\right)=\left(\begin{array}{cc}
\cos \varphi_{s} & \sin \varphi_{s} \\
-\sin \varphi_{s} & \cos \varphi_{s}
\end{array}\right)\left(\begin{array}{l}
\bar{\nu}_{s} \\
\bar{\nu}_{\tau}
\end{array}\right)
$$

where the rotation angle $\varphi_{s}$ fixes the sterile component in the atmospheric doublet $\left(0<\varphi_{s}<\pi / 2\right)$. Equation (12) then becomes

$$
\left(\begin{array}{l}
\bar{\nu}_{s} \\
\bar{\nu}_{e} \\
\bar{\nu}_{\mu} \\
\bar{\nu}_{\tau}
\end{array}\right)=\left(\begin{array}{cccc}
\frac{\cos \varphi_{s}}{\sqrt{2}} & \frac{\cos \varphi_{s}}{\sqrt{2}} & \frac{\sin \varphi_{s}}{\sqrt{2}} & -\frac{\sin \varphi_{s}}{\sqrt{2}} \\
-\frac{1}{\sqrt{2}} & \frac{1}{\sqrt{2}} & \beta & \beta \\
\beta & -\beta & \frac{1}{\sqrt{2}} & \frac{1}{\sqrt{2}} \\
\frac{\sin \varphi_{s}}{\sqrt{2}} & \frac{\sin \varphi_{s}}{\sqrt{2}} & -\frac{\cos \varphi_{s}}{\sqrt{2}} & \frac{\cos \varphi_{s}}{\sqrt{2}}
\end{array}\right)\left(\begin{array}{c}
\bar{\nu}_{0} \\
\bar{\nu}_{1} \\
\bar{\nu}_{2} \\
\bar{\nu}_{3}
\end{array}\right)
$$

which contains Eq. (12) in the specific case $\varphi_{s}=0$. We note that the LSND oscillation amplitude formula $\sin ^{2} 2 \vartheta_{L S N D}$ $=8 \beta^{2}$ holds also for the more general case of Eq. (14), and that our results are independent of the value of $\varphi_{s}$ (see Table II).
Another possible 4-neutrino model has a $(3+1)$ hierarchy; as an example for this model, here we consider the following mixing, which is also taken from [16]:

$$
\begin{aligned}
\left(\begin{array}{l}
\bar{\nu}_{e} \\
\bar{\nu}_{\mu} \\
\bar{\nu}_{\tau} \\
\bar{\nu}_{s}
\end{array}\right)= & \left.\mid \begin{array}{cccc}
\frac{1}{\sqrt{2}} & \frac{1}{\sqrt{2}} & 0 & \gamma \\
-\frac{1}{2} & \frac{1}{2} & \frac{1}{\sqrt{2}} & \delta \\
\frac{1}{2} & -\frac{1}{2} & \frac{1}{\sqrt{2}} & 0 \\
\frac{1}{2} \delta-\frac{1}{\sqrt{2}} \gamma & -\frac{1}{2} \delta-\frac{1}{\sqrt{2}} \gamma & -\frac{1}{\sqrt{2}} \delta & 1
\end{array}\right) \\
& \times\left(\begin{array}{l}
\bar{\nu}_{1} \\
\bar{\nu}_{2} \\
\bar{\nu}_{3} \\
\bar{\nu}_{4}
\end{array}\right)
\end{aligned}
$$

where the solar and atmospheric oscillations are approximately described by oscillations of three active neutrinos, and the LSND result by a coupling of $\bar{\nu}_{\mu}$ and $\bar{\nu}_{e}$ through small mixings with $\bar{\nu}_{s}$ that has a mass eigenvalue widely separated from the others $\left(\sin ^{2} 2 \vartheta_{L S N D}=4 \gamma^{2} \delta^{2}\right)$. For the $(3$ $+1)$ scenario, the constraint given by the permutation probability $p$ is not sufficient to determine the LSND oscillation amplitude $\sin ^{2} 2 \vartheta_{L S N D}$. Therefore, the constraint on $\left|U_{\mu 4}\right|^{2}$ $=\delta^{2}$ given by the CDHS and Super-K experiments will also be used, as explained later.

We should note that the mixing matrices defined in Eqs. (10)-(15) are approximations in the sense that the matrices are unitary only up to order $\mathcal{O}(\alpha, \beta, \gamma, \delta)$. These are the parameters in the mixings responsible for LSND-type oscillations, which we let float for our analysis, but we know they are small.

In order to determine the permutation factor for the mixing models, we also need to specify the neutrino mass hierarchy. In this paper, we consider for each mixing model both the cases of "normal" and "LSND-inverted" mass hierarchies. By "normal" hierarchy, here we mean that $m_{i}>m_{j}$ for $i>j$, where $m_{i}$ is the mass eigenvalue for the $\left|\bar{\nu}_{i}\right\rangle$ state. We define the "LSND-inverted" hierarchies as the ones obtained substituting $\Delta m_{L S N D} \rightarrow-\Delta m_{L S N D}$ in the normal hierarchies, without changing the hierarchy of the eventual solar and atmospheric splittings (see Table I); $\Delta m_{L S N D}$ is the neutrino mass difference responsible for LSND oscillations.

A common feature to all the mixing schemes is apparent in Table II. In the adiabatic approximation, normal mass hierarchies predict small permutation factors, while an almost complete permutation would be present for LSND-inverted hierarchies.

Given the specific neutrino mixing models considered here, we can now partially address the question whether the adiabatic approximation is applicable in this context. At a 

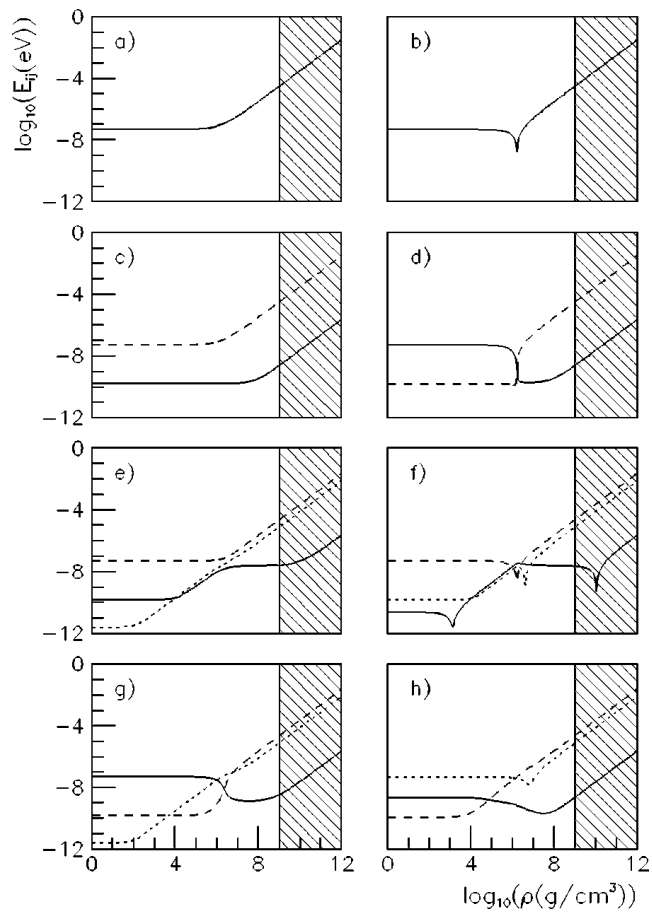

FIG. 1. Splittings between energy eigenvalues versus matter density $\rho$ for various neutrino mass and mixing models. Solid, dashed, dotted lines show the splittings $E_{12}, E_{23}, E_{34}$, respectively (see text). The local minima correspond to MSW-resonances. Model (a) normal $(1+1)$; (b) inverted $(1+1)$; (c) normal $(2+1)$; (d) inverted $(2+1)$; (e) normal $(2+2)$; (f) inverted $(2+2)$; (g) normal (3+1); (h) inverted (3+1). Apart from the inconsequential $\bar{\nu}_{\mu} \leftrightarrow \bar{\nu}_{\tau}$ one in (f), no MSW-resonances occur before the antineutrinos reach the stalled shock-wave (hatched area).

resonance, where the nonadiabaticity is maximal, this is a good approximation if the width of the resonance region is large compared with the local neutrino oscillation length. The width of the resonance is, in turn, determined by the characteristic length scale of the radial matter density variations at the resonance. While there are reliable models for the matter density profile of the progenitor star, there are still uncertainties on the profile seen by neutrinos in their freestreaming propagation.

It is now thought that neutrino heating of the protoneutron star mantle drives the supernova explosion, which would happen with $a \sim 1 \mathrm{~s}$ delay after the creation of the shock-wave, ultimately responsible for the explosion; during this delay, the shock-wave would be stalled at a radius of $\sim 200 \mathrm{~km}$ from the neutron star, corresponding to a density $\rho \sim 10^{9}-10^{10} \mathrm{~g} / \mathrm{cm}^{3}[1]$. Therefore, the density profile in the proximity of the stalled shock-wave, which is difficult to model reliably, is a potential site for nonadiabatic oscillations.

In Fig. 1 we show the energy splittings between the local neutrino energy eigenvalues $E_{i}$, as a function of matter density, for all eight neutrino models considered here. For an $n$-neutrino model, we plot $E_{i, i+1} \equiv E_{i}-E_{i+1}$, where $i$ $=1, \ldots, n-1$; the eigenvalues are ordered such that $E_{1}$ $>E_{2}>\ldots>E_{n}$. Clearly, a resonance corresponds to a local minimum in one of the curves. As can be seen from Fig. 1, all the resonances [except the inconsequential one in Fig. 1(f) between $\bar{\nu}_{\mu}$ and $\left.\bar{\nu}_{\tau}[19]\right]$ lie at densities well below the stalled shock-wave density of $\rho \sim 10^{9}-10^{10} \mathrm{~g} / \mathrm{cm}^{3}$. Therefore, the impact of level crossing between propagation eigenstates is likely to be small even where the neutrinos encounter the shock-wave.

If the $\mathrm{SN}$ neutrinos cross the Earth on their way to the detector, as for example happened for the SN 1987A $\bar{\nu}_{e}$ 's detected by the Kamiokande- 2 and IMB-3 detectors, it is also necessary to evaluate the importance of Earth matter effects in the neutrino propagation. Clearly, for neutrino oscillation models where no solar splitting is involved [for example the $(1+1)$ and $(2+1)$ models in this paper], this effect is negligible. In the models where such a splitting is allowed [i.e. the $(2+2)$ and $(3+1)$ models considered here], the situation is more complicated. However, the Earth matter effects have been shown to be small in this case as well for a large fraction of the SN $\bar{\nu}_{e}$ energy spectrum (below $\simeq 40 \mathrm{MeV}$ ) $[13,20]$, and for the sake of simplicity will not be considered further.

\section{CONSTRAINTS ON LSND FROM SN 1987A OBSERVATIONS}

Twenty $\bar{\nu}_{e}$ events from the supernova SN 1987A were observed by the Kamiokande-2 (Kam-2) and IMB-3 detectors. Kam-2 saw 12 events with an average energy of $\left\langle E_{\text {det }}\right\rangle=14.7 \mathrm{MeV}$, IMB-3 (which had a higher energy threshold than Kam-2) detected 8 events with $\left\langle E_{\text {det }}\right\rangle$ $=31.9 \mathrm{MeV}$ [21].

From a comparison of the measured energy spectra $\left(F_{\nu_{e}}\right)$ with theoretical models of neutrino emission $\left(F_{\nu_{e}}^{0}\right.$ and $\left.F_{\nu_{\mu}}^{0}\right)$, it is possible to infer the permutation factor $p$ in Eq. (1). SN 1987A observations are consistent with no-oscillations (i.e. $p=0)$. In Appendix A, we derive a conservative upper bound on $p$ of $p<0.22$ at $99 \%$ C.L., by applying a Kholmogorov-Smirnov test on the joint Kam-IMB dataset and a range of supernova neutrino emission models.

One important result of our analysis is immediately apparent from the values of the permutation factors $p$ as a function of the mixing parameters in Table II, and from the fact that the value of $p$ inferred from SN 1987A data has to be less than 0.22 at $99 \%$ C.L. The four mixing schemes considered, explaining the LSND effect via a LSND-inverted neutrino mass hierarchy, are all incompatible with SN 1987A data.

We now consider the normal hierarchy cases. For the (1 $+1),(2+1)$ and $(2+2)$ models with the mixings of Eqs. (10)-(12), the bound on the permutation factor $p$ unambigously determines the constraint on the LSND oscillation amplitude $\sin ^{2} 2 \vartheta_{L S N D}$ (see Table II). At 99\% C.L., SN 1987A data provide no constraints on the $(2+1)$ model, and a constraint which is weaker than existing bounds from the accel- 

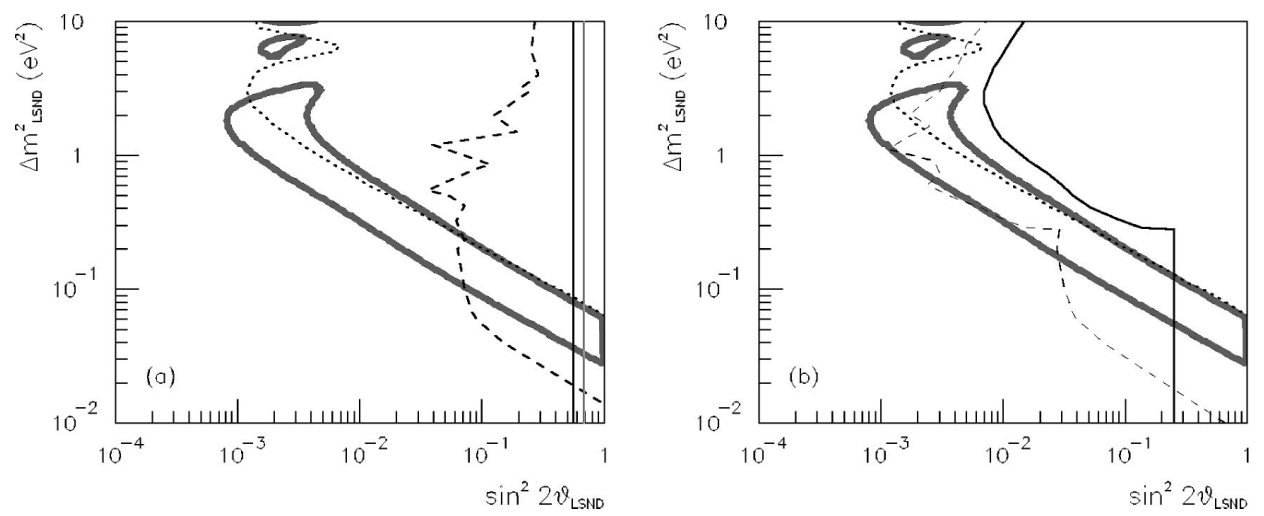

FIG. 2. 99\% C.L. LSND allowed region [7] and 99\% C.L. exclusion regions for the neutrino mixing schemes considered in the text and with normal mass hierarchy. The exclusion regions are estimated as in [26]. (a) shows the exclusion regions for the $(1+1),(2+1)$ and $(2+2)$ models, $(b)$ for the $(3+1)$ model. The exclusion regions refer to experimental data from the following experiments. (a) Dotted line: KARMEN; dashed line: Bugey; dark solid line: SN 1987A for the (2+2) model; light solid line: SN 1987 for the (1+1) model; SN 1987A data provide no constraints at $99 \%$ C.L. for the $(2+1)$ model. (b) Dotted line: KARMEN; dashed line: Bugey, CDHS and Super-K; solid line: SN 1987A, CDHS and Super-K.

erator experiment KARMEN [23] and the reactor experiment Bugey $[24,25]$ for the $(1+1)$ and $(2+2)$ models [see Fig. $2(\mathrm{a})]$. Therefore, these models are compatible with the SN 1987A data.

As already mentioned, for the $(3+1)$ model, the permutation factor does not fully determine the LSND oscillation amplitude: $\sin ^{2} 2 \vartheta_{L S N D}$ depends not only on $p$, but also on $\left|U_{\mu 4}\right|^{2}=\delta^{2}$. Here we use the $\Delta m_{L S N D}^{2}$-dependent constraints on $\delta^{2}$ from the $\nu_{\mu}$-disappearance experiments CDHS (for $\Delta m_{L S N D}^{2}>0.3 \mathrm{eV}^{2}$ ) and Super-K (for $\Delta m_{L S N D}^{2}<0.3 \mathrm{eV}^{2}$ ) [25]. Moreover, another complication arises in evaluating exclusion regions for $(3+1)$ models: given the $99 \%$ C.L. upper bounds on $\gamma^{2}=\left|U_{e 4}\right|^{2}$ from SN 1987A and $\delta^{2}=\left|U_{\mu 4}^{2}\right|$ from CDHS and Super-K, what is the $99 \%$ C.L. upper bound on $\sin ^{2} 2 \vartheta_{L S N D}=4 \gamma^{2} \delta^{2}$ ? We follow the method described in [26] to estimate this bound. The same method is applied to estimate the $99 \%$ C.L. upper limit on $\sin ^{2} 2 \vartheta_{L S N D}$ coming from Bugey (for $\gamma^{2}$ ) and CDHS and Super-K (for $\delta^{2}$ ), that is without using the SN 1987A data. The results for the (3 $+1)$ model with normal neutrino mass hierarchy and mixing given by Eq. (15) are shown in Fig. 2(b). Also for this model, we find that existing constraints (the Bugey constraint on $\delta^{2}$, in this case) are stronger than the SN 1987A one.

Table III summarizes the SN 1987A constraints obtained in this paper on the LSND allowed region, for the various neutrino mass and mixing models considered.

\section{CONCLUSIONS}

We have investigated the effect that 3- and 4-neutrino oscillation schemes would have in modifying the energy spectrum of supernova $\bar{\nu}_{e}$ 's. Throughout the paper, we apply the adiabatic approximation for the antineutrino propagation in the supernova environment and neglect Earth matter effects. Moreover, we have used our results to test the compat- ibility between the SN 1987A data and the LSND evidence for $\bar{\nu}_{\mu} \rightarrow \bar{\nu}_{e}$ oscillations.

We have provided specific relations for the permutation factor, which gives the admixture of a higher energy flux to the original $\bar{\nu}_{e}$ flux at production from $\bar{\nu}_{\mu}, \bar{\nu}_{\tau} \rightarrow \bar{\nu}_{e}$ oscillations, for various neutrino mass and mixing models. The permutation factor may be measurable with good accuracy in future supernova experiments.

Based on SN 1987A data only, which seem to indicate a small (if nonzero) value for the permutation factor, we are able to exclude all of the four models considered which would explain the LSND signal via a "LSND-inverted" neutrino mass hierarchy, as defined in the text. For the normal mass hierarchy schemes considered, SN 1987A data do not provide any stronger constraints on the LSND allowed region for oscillations than those already obtained with reactor, accelerator and atmospheric neutrinos; additional experimental input is necessary to unambiguously discern the neutrino mass and mixing properties. Undoubtedly, the detection of

TABLE III. Summary of the SN 1987A constraints on the LSND allowed region, for the various models considered in this paper; see Fig. 2 also.

\begin{tabular}{lc}
\hline \hline Model & SN 1987A constraint on \\
& LSND region (99\% C.L.) \\
\hline Normal $(1+1)$ & partially excluded [Fig. 2(a)] \\
LSND-inverted $(1+1)$ & excluded \\
Normal $(2+1)$ & unconstrained \\
LSND-inverted $(2+1)$ & excluded \\
Normal $(2+2)$ & partially excluded [Fig. 2(a)] \\
LSND-inverted (2+2) & excluded \\
Normal $(3+1)$ & partially excluded [Fig. 2(b)] \\
LSND-inverted (3+1) & excluded \\
\hline \hline
\end{tabular}


supernova neutrinos by present or near-term experiments [27] would prove very useful in this respect.

\section{ACKNOWLEDGMENTS}

We thank Kevork Abazajian, Vernon Barger, John Beacom, Nicole Bell, Steve Brice, Klaus Eitel, Lam Hui, Hitoshi Murayama and Michael Shaevitz for valuable discussions and useful suggestions. This work was supported by NSF and by the Sloan Foundation.

\section{APPENDIX: UPPER BOUNDS ON THE PERMUTATION FACTOR FROM SN 1987A DATA}

In this appendix, we discuss the statistical methodology and the physics assumptions used to estimate the upper bound on the permutation factor $p$ quoted in the text, $p$ $<0.22$ at $99 \%$ C.L. We use the same statistical methodology as in [22], that is we use the Kholmogorov-Smirnov test on the joint Kam-IMB dataset to derive the upper bound. Most of the physics assumptions are identical to those in [28].

The expected energy spectrum for the positrons, observed in the Kamiokande and IMB detectors via the reaction $\bar{\nu}_{e} p$ $\rightarrow e^{+} n$, is

$$
\begin{aligned}
n_{i}\left(E_{d e t}\right)= & \frac{N_{p, i}}{4 \pi D^{2}} \int_{0}^{\infty} d E_{+} P_{i}\left(E_{d e t}, E_{+}\right) \\
& \times \eta_{0, i}\left(E_{+}\right) \sigma_{\bar{\nu}_{e} p}\left(E_{+}+Q\right) F_{\bar{\nu}_{e}}\left(E_{+}+Q\right)
\end{aligned}
$$

where $i$ refers to either Kam or IMB, $N_{p, i}$ is the number of target protons in the detectors, $D$ the distance between the Large Magellanic Cloud and the Earth, $E_{\text {det }}\left(E_{+}\right)$is the detected (true) positron energy, $Q \equiv m_{n}-m_{p}=1.29 \mathrm{MeV} \simeq E_{\nu_{e}}$ $-E_{+}, P_{i}\left(E_{\text {det }}, E_{+}\right)$, and $\eta_{0, i}\left(E_{+}\right)$the energy resolution functions and efficiency curves taken from [28], $\sigma_{\nu_{e}}^{-}\left(E_{+}\right.$ $+Q) \propto E_{+}^{2}$ the neutrino interaction cross section taken from [5] (neglecting nuclear recoil), and finally $F_{\bar{\nu}_{e}}\left(E_{+}+Q\right)$ the neutrino flux at the detector taken from Eq. (1). We assume "unpinched" Fermi-Dirac distributions for the fluxes $F_{\nu_{\alpha}}^{0}$, $\alpha=e, \mu$, appearing in Eq. (1):

$$
F_{\bar{\nu}_{\alpha}}^{0}(E) \propto \frac{E^{2}}{\left\langle E_{\nu_{\alpha}}^{-}\right\rangle T_{\alpha}^{3}\left(e^{\left.E / T_{\alpha}+1\right)}\right.}
$$

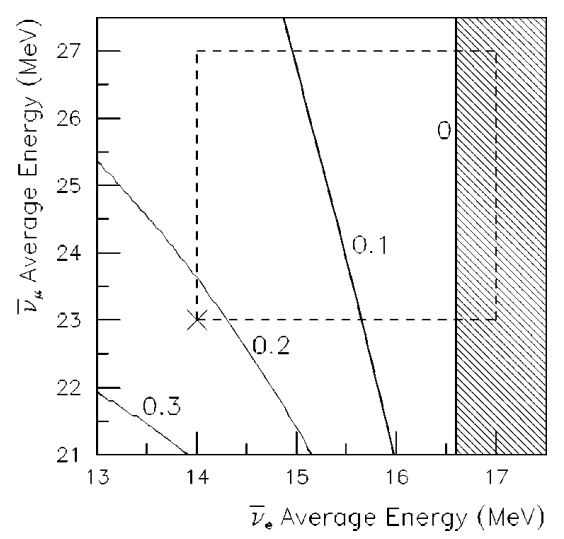

FIG. 3. Solid lines: isocontours for the upper bounds on the permutation factor $p$ at $99 \%$ C.L. obtained from SN 1987A data, as a function of the $\bar{\nu}_{e}$ and $\bar{\nu}_{\mu}$ average energies predicted at production by supernova models; rectangle with dashed border: range of energies allowed by present models; cross: model chosen to derive the (conservative) upper bound on $p$ used in the text. The region $\left\langle E_{\bar{\nu}_{e}}\right\rangle>16.6 \mathrm{MeV}$ is excluded at $99 \%$ C.L. for all values of $p$.

where $\left\langle E_{\bar{\nu}_{\alpha}}\right\rangle \simeq 3.15 T_{\alpha}$ at the denominator ensures energy equipartition.

The cumulative distribution function used for the Kholmogorov-Smirnov test is

$$
\mathcal{F}\left(E_{\text {det }}\right)=\int_{0}^{E_{d e t}} d E\left[n_{\text {Kam }}(E)+n_{I M B}(E)\right] .
$$

Figure 3 shows the upper bound on the permutation factor $p$ obtained from SN 1987A data, at $99 \%$ C.L., as a function of the average energies $\left\langle E_{\bar{\nu}_{e}}\right\rangle,\left\langle E_{\bar{\nu}_{\mu}}\right\rangle$. As expected, the bound becomes more stringent for supernova models in which the neutrino average energies are higher. SN 1987A data are incompatible at $99 \%$ C.L. with all supernova neutrino models predicting $\left\langle E_{\bar{\nu}_{e}}\right\rangle>16.6 \mathrm{MeV}$, for all values of $p$ and $\left\langle E_{\bar{\nu}_{\mu}}\right\rangle$. We adopt a conservative approach, and quote as the upper bound on $p$ the largest value for supernova neutrino models in the range $14<\left\langle E_{v_{e}}^{-}\right\rangle<17 \mathrm{MeV}, 23<\left\langle E_{\nu_{\mu}}^{-}\right\rangle<27 \mathrm{MeV}$, that is the one corresponding to $\left\langle E_{\bar{\nu}_{e}}\right\rangle=14 \mathrm{MeV},\left\langle E_{\bar{\nu}_{\mu}}\right\rangle$ $=23 \mathrm{MeV}$ (cross in Fig. 3).
[1] A. Burrows et al., Astrophys. J. 539, 865 (2000).

[2] H.-T. Janka, in Proceedings of the Vulcano Workshop 1992, Frontier objects in astrophysics and particle physics, pp. 345374.

[3] H.-T. Janka, Astropart. Phys. 3, 377 (1995).

[4] G.G. Raffelt, Nucl. Phys. B (Proc. Suppl.) 110, 254 (2002).

[5] P. Vogel and J.F. Beacom, Phys. Rev. D 60, 053003 (1999).

[6] There has been some discussion whether to interpret the first
Kam-2 event as $\bar{\nu}_{e}$ or $\nu_{e}$, see for example H. Murayama and T. Yanagida, Phys. Lett. B 520, 263 (2001). The Kam-2 Collaboration prefers to interpret all their 12 events as $\bar{\nu}_{e}$ interactions; see [21] for a discussion.

[7] LSND Collaboration, A. Aguilar et al., Phys. Rev. D 64, 112007 (2001).

[8] MiniBooNE Collaboration, A. Bazarko, Nucl. Phys. B (Proc. Suppl.) 91, 210 (2000). 
[9] B. Kayser, hep-ph/0104147.

[10] D.O. Caldwell, G.M. Fuller, and Y.Z. Qian, Phys. Rev. D 61, 123005 (2000).

[11] Y.Z. Qian et al., Phys. Rev. Lett. 71, 1965 (1993).

[12] F.J. Botella, C.S. Lim, and W.J. Marciano, Phys. Rev. D 35, 896 (1987).

[13] A.S. Dighe and A.Y. Smirnov, Phys. Rev. D 62, 033007 (2000).

[14] G. Barenboim et al., hep-ph/0108199.

[15] G. Barenboim, A.S. Dighe, and S. Skadhauge, Phys. Rev. D 65, 053001 (2002).

[16] V. Barger et al., Phys. Rev. D 63, 033002 (2001).

[17] SNO Collaboration, Q.R. Ahmad et al., Phys. Rev. Lett. 89, 011301 (2002).

[18] V. Barger et al., Phys. Rev. D 58, 093016 (1998).

[19] Let us see what the effect of a possible violation of adiabaticity between the two highest energy eigenstates, $\bar{\nu}_{\mu}$ and $\bar{\nu}_{\tau}$ at high matter densities, in the case of a LSND-inverted $(2+2)$ model [Fig. 1(f)] is on $p_{e \rightarrow e}$ and $p_{\mu \rightarrow e}+p_{\tau \rightarrow e}$. Violation of adiabaticity would result in a non-vanishing hopping probability $p_{\text {hop }}$ between these two local Hamiltonian eigenstates. Clearly, $p_{e \rightarrow e}$ is unaffected, and also $p_{\mu \rightarrow e}+p_{\tau \rightarrow e}=p_{h o p}\left|U_{e 0}^{e v o l}\right|^{2}+(1$ $\left.-p_{\text {hop }}\right)\left|U_{e 1}^{\text {evol }}\right|^{2}+\left(1-p_{\text {hop }}\right)\left|U_{e 0}^{e v o l}\right|^{2}+p_{\text {hop }}\left|U_{e 1}^{e v o l}\right|^{2}=\left|U_{e 0}^{e v o l}\right|^{2}$
$+\left|U_{e 1}^{e v o l}\right|^{2}$ has the same value as in the adiabatic case.

[20] C. Lunardini and A.Y. Smirnov, Nucl. Phys. B616, 307 (2001).

[21] M. Koshiba, Phys. Rep. 220, 229 (1992).

[22] A.Y. Smirnov, D.N. Spergel, and J.N. Bahcall, Phys. Rev. D 49, 1389 (1994).

[23] KARMEN Collaboration, B. Armbruster et al., Phys. Rev. D 65, 112001 (2002); K. Eitel (private communication).

[24] Y. Declais et al., Nucl. Phys. B434, 503 (1995).

[25] S.M. Bilenky et al., Phys. Rev. D 54, 1881 (1996); 60, 073007 (1999).

[26] O.L.G. Peres and A.Y. Smirnov, Nucl. Phys. B599, 3 (2001).

[27] J.F. Beacom and P. Vogel, Phys. Rev. D 58, 053010 (1998); 58, 093012 (1998); L. Cadonati, F.P. Calaprice, and M.C. Chen, Astropart. Phys. 16, 361 (2002); P. Vogel, Prog. Part. Nucl. Phys. 48, 29 (2002); W. Fulgione, Nucl. Phys. B (Proc. Suppl.) 70, 469 (1999); F. Halzen, J.E. Jacobsen, and E. Zas, Phys. Rev. D 53, 7359 (1996); M. K. Sharp, J. F. Beacom, and J. A. Formaggio, ibid. 66, 013012 (2002).

[28] B. Jegerlehner, F. Neubig, and G. Raffelt, Phys. Rev. D 54, 1194 (1996) 


\subsection{Short-Baseline Neutrino Experiments and the Number of Neutrino Species}

As discussed in the previous Chapter, if the LSND observation of the $\bar{\nu}_{e}$ excess in a $\bar{\nu}_{\mu}$ neutrino beam is to be interpreted as $\bar{\nu}_{\mu} \rightarrow \bar{\nu}_{e}$ oscillations, then additional, sterile neutrino species may be required to explain all of the experimental evidence on neutrino oscillations. Within these assumptions, short-baseline neutrino oscillation experiments provide constraints on the number of light sterile neutrino species, their masses and their mixings.

In the following paper (see Ref. [70] and references therein), written in collaboration with J. Conrad and M. Shaevitz, the constraints on sterile neutrino models from a combined analysis of several accelerator-based and reactor-based searches for $\nu_{\mu} \rightarrow \nu_{\mu}, \nu_{e} \rightarrow \nu_{\phi}$, and $\nu_{\mu} \rightarrow \nu_{e}$ oscillations at short baselines are presented. Neutrino models involving both one and two additional sterile neutrinos are studied. The first main result of this analysis is that $(3+2)$ sterile neutrino models are significantly favored over $(3+1)$ models. The second main result of the analysis is an estimate of the most likely and allowed values for the neutrino mass splittings and mixing matrix elements affecting the three oscillation channels listed above at short baselines, for both $(3+1)$ and $(3+2)$ models.

These results provide some guidance for present and future short-baseline oscillation searches, as well as for neutrino model building. In particular, this phenomenological work is of direct relevance to the MiniBooNE $\nu_{\mu}$ disappearance analysis presented later in this thesis, and shows that sterile neutrino models may predict large (up to $\sim 30 \%) \nu_{\mu}$ disappearance in the $\Delta m^{2}$ range probed by the MiniBooNE experiment. 
PHYSICAL REVIEW D, VOLUME 70, 073004

\title{
Combined analysis of short-baseline neutrino experiments in the $(3+1)$ and $(3+2)$ sterile neutrino oscillation hypotheses
}

\author{
M. Sorel, ${ }^{*}$ J. M. Conrad, ${ }^{\dagger}$ and M. H. Shaevitz \\ Department of Physics, Columbia University, New York, New York 10027, USA \\ (Received 21 April 2004; published 12 October 2004)
}

\begin{abstract}
We investigate adding two sterile neutrinos to resolve the apparent tension existing between shortbaseline neutrino oscillation results and CPT-conserving, four-neutrino oscillation models. For both $(3+1)$ and $(3+2)$ models, the level of statistical compatibility between the combined dataset from the null short-baseline experiments Bugey, CHOOZ, CCFR84, CDHS, KARMEN, and NOMAD, on the one hand; and the LSND dataset, on the other, is computed. A combined analysis of all seven shortbaseline experiments, including LSND, is also performed, to obtain the favored regions in neutrino mass and mixing parameter space for both models. Finally, four statistical tests to compare the $(3+1)$ and the $(3+2)$ hypotheses are discussed. All tests show that $(3+2)$ models fit the existing shortbaseline data significantly better than $(3+1)$ models.
\end{abstract}

PACS numbers: 14.60.Pq, 12.15.Ff, 14.60.St

\section{INTRODUCTION}

There currently exist three experimental signatures for neutrino oscillations. The two signatures seen originally in solar and atmospheric neutrinos have been verified by several experiments, including experiments carried out with accelerator and nuclear reactor sources. The results on atmospheric neutrinos can be explained by $\nu_{\mu}$ disappearance due to oscillations [1-3], while those on solar neutrinos can be explained by $\nu_{e}$ oscillations [4,5]. The third signature is $\bar{\nu}_{e}$ appearance in a $\bar{\nu}_{\mu}$ beam, observed by the short-baseline, accelerator-based LSND experiment at Los Alamos [6]. This signature is strong from a statistical point of view, being a $3.8 \sigma$ excess, but further confirmation by an independent experiment is necessary. The MiniBooNE experiment at Fermilab will be able to confirm or refute the LSND signature in the near future, with an experimental setup providing different systematics and higher statistics than LSND [7].

Taken at face value, the three experimental signatures point to three independent mass splittings. Three neutrino masses do not appear to be able to explain all of the three signatures [8,9] (see, however, [10]). One way to solve this puzzle is to introduce different mass spectra for the neutrino and antineutrino sector, thereby requiring CPT violation but no extra neutrino generations [11]. Another possibility is to add additional neutrinos with no standard weak couplings, often called "sterile neutrinos."

In this paper we assume CPT and CP invariance, and we explore the possibility of adding one- or two-neutrino generations beyond the three active flavors assumed by the Standard Model. We focus on extensions of the neutrino sector where the addition of fourth and fifth mass

\footnotetext{
*Electronic address: sorel@ nevis.columbia.edu

†lectronic address: conrad@nevis.columbia.edu

†Electronic address: shaevitz@nevis.columbia.edu
}

eigenstates are responsible for the high $\Delta m^{2}$ LSND oscillations, and the three lower mass states explain solar and atmospheric oscillations. When only one sterile neutrino is added, these models are labeled as $(3+1)$. The flavor content of the four-neutrino mass eigenstates for these models is schematically shown in Fig. 1(a). The $(3+$ 1) hierarchy in Fig. 1(a) is as opposed to the $(2+2)$ hierarchy, where the solar and atmospheric mass splittings are separated from each other by the LSND $\Delta m^{2}$. The $(2+2)$ models require a different global analysis from the one discussed in this paper. The simplest $(2+$ 2) models appear to be only marginally consistent with neutrino oscillations data $[8,12]$, even though more general $(2+2)$ mass and mixing scenarios might represent a viable solution to explain solar, atmospheric, and LSND oscillations [13].

The $(3+1)$ models are motivated by the criterion of simplicity in physics, introducing the most minimal extension to the Standard Model that explains the experimental evidence. However, theories invoking sterile neutrinos to explain the origin of neutrino masses do not necessarily require only one sterile neutrino. Indeed, many popular realizations of the see-saw mechanism introduce three right-handed neutrino fields [1416]. In particular, $(3+2)$ neutrino mass and mixing models can be obtained in several see-saw mechanisms [17]. From the phenomenological point of view, it is our opinion that two- and three-sterile neutrino models should also be considered and confronted with existing experimental results. In this paper, we consider the results from the short-baseline experiments Bugey [18], CCFR84 [19], CDHS [20], CHOOZ [21], KARMEN [22], LSND [6], and NOMAD [23], and examine how well $(3+1)$ and $(3+2)$ models agree with data. A schematic diagram for $(3+2)$ models is shown in Fig. 1(b). We do not consider $(3+3)$ models in this paper. From our initial studies, we believe that the phenomenology of a $(3+3)$ model is similar to a $(3+2)$ model. 
4

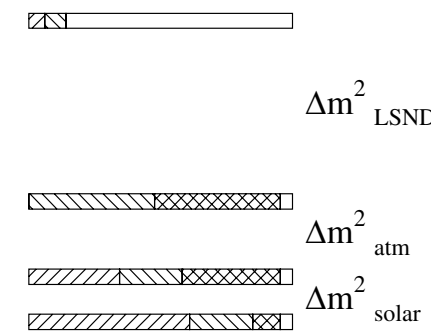

a)
5

4

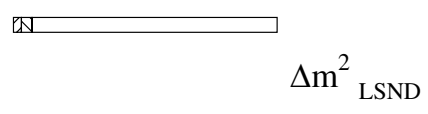

3 लाIIIIVx

$\Delta \mathrm{m}^{2}$ atm

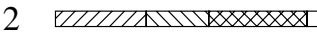

$\Delta \mathrm{m}^{2}$ solar

b)

FIG. 1. Flavor content of neutrino mass eigenstates in $(3+1)$ models $(a)$, and $(3+2)$ models $(b)$. Neutrino masses increase from bottom to top. The $\nu_{e}$ fractions are indicated by right-leaning hatches, the $\nu_{\mu}$ fractions by left-leaning hatches, the $\nu_{\tau}$ fractions by crosshatches, and the $\nu_{s}$ fractions by no hatches. The flavor contents shown are schematic only.

The paper is organized as follows. In Sec. II, we specify the neutrino oscillations formalism used in this analysis to describe $(3+1)$ and $(3+2)$ short-baseline oscillations. In Sec. III and IV, we present the results obtained for the $(3+1)$ and $(3+2)$ models, respectively. For both models, we first derive the level of compatibility between the null short-baseline (NSBL) experiments and LSND. Second, we perform a combined analysis of all seven short-baseline experiments (including LSND) to derive the preferred regions in neutrino mass and mixing parameter space. In Sec. V, we discuss four statistical tests to compare the $(3+1)$ and $(3+2)$ hypotheses. In Sec. VI, we briefly mention other experimental constraints on $(3+1)$ and $(3+2)$ models. In the Appendix, we describe the physics and statistical assumptions used in the analysis to describe the short-baseline experiments.

\section{NEUTRINO OSCILLATIONS FORMALISM}

Under the assumptions of CP and CPT invariance, the probability for a neutrino, produced with flavor $\alpha$ and energy $E$, to be detected as a neutrino of flavor $\beta$ after travelling a distance $L$, is [24]

$$
P\left(\nu_{\alpha} \rightarrow \nu_{\beta}\right)=\delta_{\alpha \beta}-4 \sum_{j>i}^{n} U_{\alpha, j} U_{\beta, j} U_{\alpha, i} U_{\beta, i} \sin ^{2} x_{j i}
$$

where $\alpha=e, \mu, \tau, s$ ( $s$ being the sterile flavor); $U$ is the unitary neutrino mixing matrix; $x_{j i} \equiv 1.27 \Delta m_{j i}^{2} L / E$; $\Delta m_{j i}^{2} \equiv m_{j}^{2}-m_{i}^{2}$; and $n$ is the number of neutrino generations. Neglecting CP-violating phases, there are in general $(n-1)$ independent mass splittings, and $n^{2}-$ $n-n(n-1) / 2$ independent mixing matrix elements. The situation simplifies considerably by considering short-baseline (SBL) data only. In this case, it is a good approximation to assume $x_{21}=x_{32}=0$, and only $(n-3)$ independent mass splittings are present. Moreover, given the set of SBL experiments considered, the number of mixing matrix elements probed is only $2(n-3)$, as we show now for the $(3+1)$ and $(3+2)$ cases.

For $(3+1)$ models, $n=4$, and only one mass splitting $\Delta m^{2} \equiv \Delta m_{41}^{2} \simeq \Delta m_{42}^{2} \simeq \Delta m_{43}^{2}$ appears in the oscillation formula: this is sometimes referred to as to the "quasi two neutrino approximation," or "one mass scale dominance" [25]. Using the unitarity properties of the mixing matrix, we can rewrite Eq. (1) for $(3+1)$ models in a more convenient way

$$
P\left(\nu_{\alpha} \rightarrow \nu_{\beta}\right)=\delta_{\alpha \beta}-4 U_{\alpha 4} U_{\beta 4}\left(\delta_{\alpha \beta}-U_{\alpha 4} U_{\beta 4}\right) \sin ^{2} x_{41},
$$

which depends on the mass splitting $\left(\Delta m_{41}^{2}\right)$ and mixing parameters $\left(U_{\alpha 4}, U_{\beta 4}\right)$ of the fourth generation only. Since the two neutrino approximation is satisfied in the $(3+1)$ case, we can express Eq. (2) in the usual forms

$$
\begin{gathered}
P\left(\nu_{\alpha} \rightarrow \nu_{\beta}\right)=\sin ^{2} 2 \theta_{\alpha \beta} \sin ^{2} x_{41}, \alpha \neq \beta, \\
P\left(\nu_{\alpha} \rightarrow \nu_{\alpha}\right)=1-\sin ^{2} 2 \theta_{\alpha \alpha} \sin ^{2} x_{41},
\end{gathered}
$$

where Eq. (3) applies to an oscillation appearance measurement, Eq. (4) to a disappearance measurement.

In this paper, we use the data from the Bugey, CCFR84, CDHS, CHOOZ, KARMEN, LSND, and NOMAD experiments. Bugey and $\mathrm{CHOOZ}$ data constrain $\nu_{e}$ disappearance; CCFR84 and CDHS data constrain $\nu_{\mu}$ disappearance; and KARMEN, LSND, and NOMAD data constrain $\nu_{u} \rightarrow \nu_{e}$ oscillations. Therefore, from Eqs. (2)-(4), the experiments constrain the following combinations of $(3+1)$ mixing parameters:

(i) Bugey, CHOOZ: $\sin ^{2} 2 \theta_{e e} \equiv 4 U_{e 4}^{2}\left(1-U_{e 4}^{2}\right)$;

(ii) CCFR84, CDHS: $\sin ^{2} 2 \theta_{\mu \mu} \equiv 4 U_{\mu 4}^{2}\left(1-U_{\mu 4}^{2}\right)$;

(iii) KARMEN, LSND, NOMAD: $\sin ^{2} 2 \theta_{\mu e} \equiv$ $4 U_{e 4}^{2} U_{\mu 4}^{2}$.

In $(3+1)$ models, the tension between the experimental results comes about because Bugey, CHOOZ, CCFR84, CDHS, KARMEN, and NOMAD limit the two 
independent mixing matrix parameters $U_{e 4}$ and $U_{\mu 4}$ to be small, whereas LSND demands nonzero values.

In $(3+2)$ models, we introduce two sterile neutrinos. Using Eq. (1) and the unitarity of the mixing matrix, the $(3+2)$ neutrino oscillation probability formula can be written

$$
\begin{aligned}
P\left(\nu_{\alpha}\right. & \left.\rightarrow \nu_{\beta}\right)=\delta_{\alpha \beta}-4\left[\left(\delta_{\alpha \beta}-U_{\alpha 4} U_{\beta 4}-U_{\alpha 5} U_{\beta 5}\right)\left(U_{\alpha 4} U_{\beta 4} \sin ^{2} x_{41}+U_{\alpha 5} U_{\beta 5} \sin ^{2} x_{51}\right)+U_{\alpha 4} U_{\alpha 5} U_{\beta 4} U_{\beta 5} \sin ^{2} x_{54}\right]= \\
& =\delta_{\alpha \beta}+4\left[U_{\alpha 4}^{2}\left(U_{\beta 4}^{2}-\delta_{\alpha \beta}\right) \sin ^{2} x_{41}+U_{\alpha 5}^{2}\left(U_{\beta 5}^{2}-\delta_{\alpha \beta}\right) \sin ^{2} x_{51}+U_{\alpha 4} U_{\beta 4} U_{\alpha 5} U_{\beta 5}\left(\sin ^{2} x_{41}+\sin ^{2} x_{51}-\sin ^{2} x_{54}\right)\right],
\end{aligned}
$$

which in our case depends on two independent mass splittings $\left(\Delta m_{41}^{2}, \Delta m_{51}^{2}\right)$ and four independent mixing matrix parameters $\left(U_{\alpha 4}, U_{\alpha 5}\right.$, with $\left.\alpha=e, \mu\right)$. Equation (2) can be recovered from Eq. (5) by requiring $U_{\alpha 5}=U_{\beta 5}=0$. In $(3+2)$ models, the quasi two neutrino approximation is not valid, since there are three distinct $\Delta m^{2}$ values contributing in the oscillation formula: $\Delta m_{41}^{2}, \Delta m_{51}^{2}$, and $\Delta m_{54}^{2}$, and therefore three distinct oscillation amplitudes: $\left(\sin ^{2} 2 \theta_{\alpha \beta}\right)_{41},\left(\sin ^{2} 2 \theta_{\alpha \beta}\right)_{51}$, and $\left(\sin ^{2} 2 \theta_{\alpha \beta}\right)_{54}$.

We now comment on the Monte Carlo method used to apply the above oscillation formalism to the analyses presented in this paper. We require the neutrino mass splittings to be in the range $0.1 \mathrm{eV}^{2} \leq \Delta m_{41}^{2}, \Delta m_{51}^{2} \leq$ $100 \mathrm{eV}^{2}$, with $\Delta m_{51}^{2} \geq \Delta m_{41}^{2}$. Each mass splitting range is analyzed over a 200 point grid, uniformly in $\log _{10} \Delta m^{2}$. In Sec. VI, we briefly discuss why large mass splittings are not necessarily in contradiction with cosmological (and other) data. The values of the mixing parameters, $U_{e 4}$, $U_{\mu 4}, U_{e 5}$, and $U_{\mu 5}$, are randomly generated over a fourdimensional space satisfying the four requirements: $U_{e i}^{2}+U_{\mu i}^{2} \leq 0.5, U_{\alpha 4}^{2}+U_{\alpha 5}^{2} \leq 0.5$, where $i=4,5, \alpha=$ $e, \mu$. These four inequalities are introduced to account for the fact that large electron and muon flavor fractions in the fourth and fifth mass eigenstates are not allowed by solar and atmospheric neutrino data. In principle, since the CDHS constraint on $\nu_{\mu}$ disappearance vanishes for $\Delta m^{2} \simeq 0.3 \mathrm{eV}^{2}$, as shown in the Appendix, the upper limit on $\nu_{\mu}$ disappearance from atmospheric neutrino experiments above the atmospheric $\Delta m^{2}$ should be considered instead. In this paper, we do not reconstruct the likelihood for atmospheric data that would give the exclusion region for $\nu_{\mu}$ disappearance in the range $\Delta m_{\text {atm }}^{2} \ll \Delta m^{2}<0.3 \mathrm{eV}^{2}$. However, the effect that the atmospheric constraints would have on our results is expected to be small. For example, in Ref. [26], Bilenky et al. use the atmospheric up-down asymmetry to derive the upper limit $U_{\mu 4}^{2}<0.55$ at $90 \% \mathrm{CL}$ for $(3+$ 1) models, which is satisfied by our initial requirements $U_{e 4}^{2}+U_{\mu 4}^{2}<0.5, U_{\mu 4}^{2}+U_{\mu 5}^{2}<0.5$. A more recent analysis [27] of atmospheric neutrino data using the full zenith angle distribution provides a tighter constraint on $\sin ^{2} \theta_{\mu \mu}$ than the one given in Ref. [26]; the impact of this additional constraint on our SBL analysis is discussed in Sections III and VI. Finally, from Eqs. (2), it is clear that the relative sign of both $U_{e 4}$ and $U_{\mu 4}$ cannot be inferred in $(3+1)$ oscillations. Similarly, from Eq. (5), the only physically observable relative sign between mixing parameters in CP-conserving $(3+2)$ models is $\operatorname{sign}\left(U_{e 4} U_{\mu 4} U_{e 5} U_{\mu 5}\right)$; therefore, this is the only sign related to mixing parameters that we randomly generate in the analysis.

Throughout the paper, we make use of the Gaussian approximation in determining allowed regions in parameter space. In general, this means that the regions of quoted confidence level are the ones enclosed by contours of constant $\chi^{2}$ values, whose differences with respect to the best-fit $\chi^{2}$ value depend on the number of free parameters in the model [28]. In the text, we use the symbol $\delta$ to denote the values of the confidence levels derived in this way. As pointed out in [29], this approach should be considered approximate, as it may provide regions in parameter space of both higher and lower confidence than the one quoted. Regions of higher confidence than the quoted value may result from the presence of highly correlated parameters. Regions of lower confidence may result from the presence of fast oscillatory behavior of the oscillation probability formula, Eq. (1).

\section{RESULTS FOR $(3+1)$ MODELS}

This section, like the next one on $(3+2)$ models, consists of two parts. First, we quantify the statistical compatibility between the NSBL and LSND results, following a method described in [30,31], originally proposed to establish the compatibility between the LSND and KARMEN results. Second, we perform a combined analysis of the NSBL and LSND datasets, to obtain the favored regions in neutrino mass and mixing parameter space.

\section{A. Statistical compatibility between NSBL and LSND}

Many analyses of the NSBL experiments within $(3+1)$ models have concluded that the allowed LSND region is largely excluded [32-34]. Here, we repeat this study with two purposes. First, we use this study to give context to our discussion of the basic model and techniques which will be expanded in later sections. Second, we demonstrate that our fit, which forms the basis of our new results for $(3+2)$ models, reproduces the expected $(3+1)$ exclusion region. For a discussion of the physics and statistical assumptions used to describe the short-baseline 


\section{SOREL, J. M. CONRAD, AND M. H. SHAEVITZ}

experiments used in the analysis, the reader should refer to the Appendix.

In this section, the NSBL and LSND datasets are analyzed separately, providing two independent allowed regions in $\left(\sin ^{2} 2 \theta_{\mu e}, \Delta m^{2}\right)$ space. The level of statistical compatibility between the two datasets can be determined by studying to what degree the two allowed regions overlap, as will be quantified later in this section.

For each randomly generated $(3+1)$ model, we calculate the values for the $\chi^{2}$ functions $\chi_{\mathrm{NSBL}}^{2}$ and $\chi_{\mathrm{LSND}}^{2}$, where $\chi_{\mathrm{NSBL}}^{2}$ is defined as

$$
\begin{aligned}
\chi_{\mathrm{NSBL}}^{2} \equiv & \chi_{\text {Bugey }}^{2}+\chi_{\mathrm{CHOOZ}}^{2}+\chi_{\mathrm{CCFR} 84}^{2}+\chi_{\mathrm{CDHS}}^{2} \\
& +\chi_{\text {KARMEN }}^{2}+\chi_{\text {NOMAD. }}^{2}
\end{aligned}
$$

For the analysis described in this section, the NSBL and LSND allowed regions are obtained using two different algorithms, reflecting the fact that the NSBL dataset provides upper limits on oscillations, while the LSND dataset points to non-null oscillations.

The NSBL allowed regions at various confidence levels $\delta_{\mathrm{NSBL}}$ are obtained via a raster scan algorithm [29]. Let $\chi_{\mathrm{NSBL}}^{2}$ be the $\chi^{2}$ value for the particular model and $\left(\chi_{\mathrm{NSBL}}^{2}\right)_{\min , \Delta m^{2}}$ be the minimum $\chi^{2}$ for the $\Delta m^{2}$ value considered. For example, our quoted $95 \%$ CL upper limit on $\sin ^{2} 2 \theta_{\mu e}$ is given by the maximum value for the product $4 U_{e 4}^{2} U_{\mu 4}^{2}$ chosen among the models which satisfy the inequality $\chi_{\mathrm{NSBL}}^{2}-\left(\chi_{\mathrm{NSBL}}^{2}\right)_{\min , \Delta m^{2}}<5.99$. The value of 5.99 units of $\chi^{2}$ is chosen because there are two free

\section{PHYSICAL REVIEW D 70073004}

parameters $U_{e 4}, U_{\mu 4}$ for $(3+1)$ models with fixed $\Delta m^{2}$. We note that even for the NSBL dataset, the parameters $U_{e 4}, U_{\mu 4}$ can be correlated, since the KARMEN and NOMAD results probe a combination of the two parameters.

The LSND allowed regions at various confidence levels $\delta_{\text {LSND }}$ are obtained via a global scan algorithm [29]. For example, for $\delta_{\text {LSND }}=0.95$ we require $\chi_{\text {LSND }}^{2}-$ $\left(\chi_{\mathrm{LSND}}^{2}\right)_{\min }<5.99$, where $\left(\chi_{\mathrm{LSND}}^{2}\right)_{\min }$ is now the global LSND $\chi^{2}$ minimum value, considering all possible $\Delta m^{2}$ values. The LSND allowed region is computed for two free parameters as for the NSBL case, but the parameters are now $\Delta m^{2}$ and $U_{\mu 4} U_{e 4}$, as opposed to $U_{\mu 4}$ and $U_{e 4}$. Compared to the NSBL case, the number of free parameters is reduced by one because the LSND $\bar{\nu}_{\mu} \rightarrow \bar{\nu}_{e}$ search only probes the product $U_{\mu 4} U_{e 4}$ and not the two mixing matrix elements individually, and it is increased by one because the allowed region is now obtained by scanning over all possible $\Delta m^{2}$ values.

The regions allowed in $\left(\sin ^{2} 2 \theta_{\mu e}, \Delta m^{2}\right)$ parameter space by both the NSBL and LSND datasets are shown in Fig. 2(a). The NSBL allowed regions shown are twodimensional projections of three-dimensional allowed regions in $\left(\Delta m^{2}, U_{e 4}, U_{\mu 4}\right)$ space. The NSBL results alone allow the regions to the left of the solid gray, dotted black, and solid black lines in the Fig. 2(a), at a confidence level $\delta_{\mathrm{NSBL}}=0.90,0.95,0.99$, respectively. In Fig. 2(a), the $\delta_{\text {LSND }}=0.90,0.95,0.99 \mathrm{CL}$ allowed regions obtained by our analysis for LSND data are also
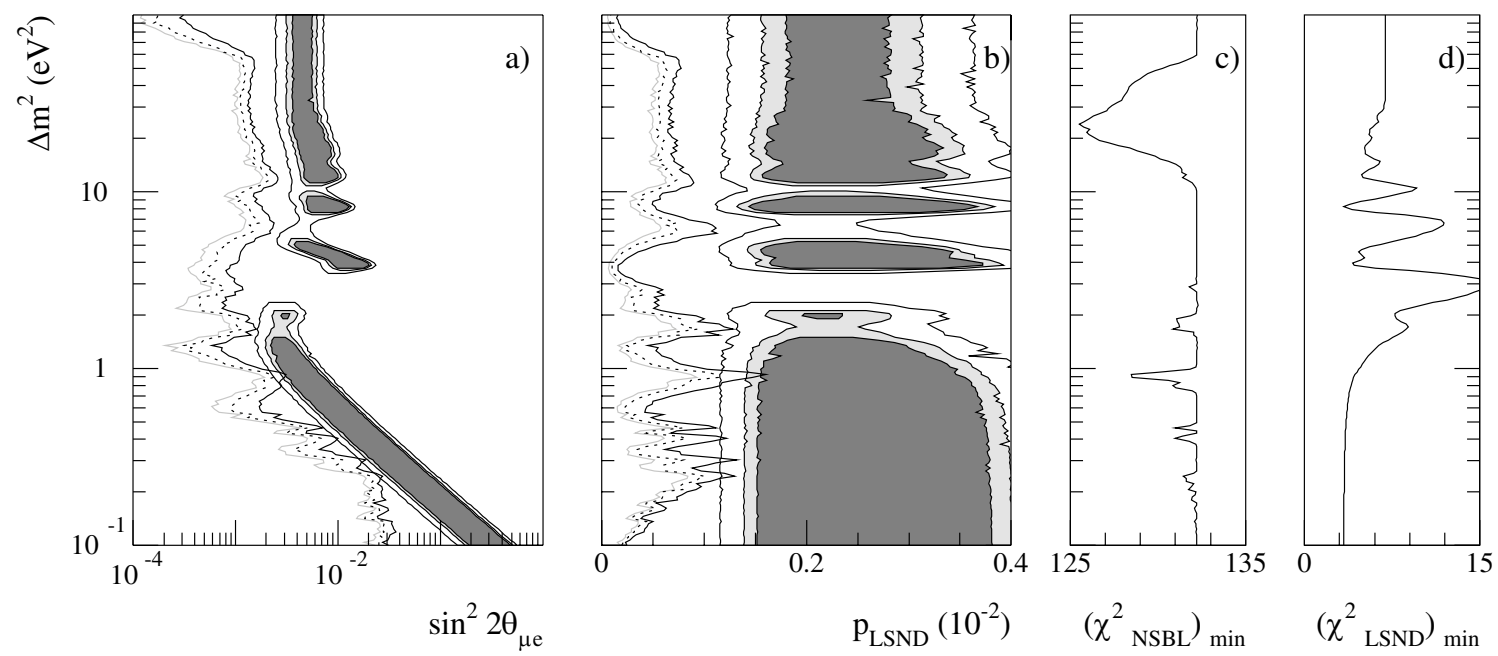

FIG. 2. Compatibility between the NSBL and LSND datasets in $(3+1)$ models. Figure (a) shows the $90 \%$ ( gray solid line), $95 \%$ (black dotted line), and 99\% (black solid line) CL exclusion curves in $\left(\sin ^{2} 2 \theta_{\mu e}, \Delta m^{2}\right)$ space for $(3+1)$ models, considering the null short-baseline (NSBL) experiments Bugey, CCFR84, CDHS, CHOOZ, KARMEN, and NOMAD. Figure (b) also shows the $90 \%, 95 \%$, and $99 \%$ CL allowed regions by our analysis of LSND data. Figure (b) is as Figure (a), but in $\left(p_{\text {LSND }}, \Delta m^{2}\right)$ space, where $p_{\text {LSND }}$ is the LSND oscillation probability (see text for the definition). Figures (c) and (d) show the minimum $\chi^{2}$ values as a function of $\Delta m^{2}$ for the NSBL and LSND datasets (143 and three d.o.f., respectively). 
COMBINED ANALYSIS OF SHORT-BASELINE...

shown, as dark gray shaded, light gray shaded, and white areas, respectively. We find no overlap between the two individual 95\% CL allowed regions; on the other hand, there is overlap between the two 99\% CL regions.

Figure 2(b) shows the same $(3+1)$ allowed regions as Fig. 2(a) but in the $\left(p_{\text {LSND }}, \Delta m^{2}\right)$ plane, where $p_{\text {LSND }}$ is defined as the $\nu_{\mu} \rightarrow \nu_{e}$ oscillation probability averaged over the LSND $L / E$ distribution

$$
p_{\mathrm{LSND}} \equiv\left\langle P\left(\nu_{\mu} \rightarrow \nu_{e}\right)\right\rangle,
$$

where $P\left(\nu_{\mu} \rightarrow \nu_{e}\right)$ is given by Eq. (1) for $\alpha=\mu, \beta=e$, and is a function of all the mass and mixing parameters of the oscillation model under consideration. This has the obvious disadvantage of being a quantity dependent upon the specifics of a certain experiment, as opposed to a universal variable such as $\sin ^{2} 2 \theta_{\mu e}=4 U_{\mu 4}^{2} U_{e 4}^{2}$. However, $p_{\text {LSND }}$ has the advantage of being unambiguously defined for any number of neutrino generations, and thus is useful in discussing $(3+2)$ models later in this paper. As stated previously, the oscillation probability estimator $\sin ^{2} 2 \theta_{\mu e}=4 U_{\mu 4}^{2} U_{e 4}^{2}$ cannot be used when more than one $\Delta m^{2}$ value affects the oscillation probability, as is the case for $(3+2)$ models. A second advantage of using $p_{\text {LSND }}$ instead of $\sin ^{2} 2 \theta_{\mu e}$ as the oscillation probability estimator, is that the allowed values for $p_{\text {LSND }}$ inferred from the LSND result tend to be almost $\Delta m^{2}-$ independent (see gray-shaded areas in Fig. 2(b)), as expected for an almost pure counting experiment such as LSND. The oscillation probability reported by the LSND collaboration [6] is $p_{\mathrm{LSND}}=(0.264 \pm 0.067 \pm 0.045) \%$, and agrees well with our result of Fig. 2(b).

Figure 2(c) shows the values for $\left(\chi_{\mathrm{NSBL}}^{2}\right)_{\min }$ as a function of $\Delta m^{2}$. The number of degrees of freedom is 143. As discussed in the Appendix, the dip in $\left(\chi_{\mathrm{NSBL}}^{2}\right)_{\min }$ at $\Delta m^{2} \simeq$ $0.9 \mathrm{eV}^{2}$ is due to Bugey data preferring $U_{e 4} \neq 0$ values,

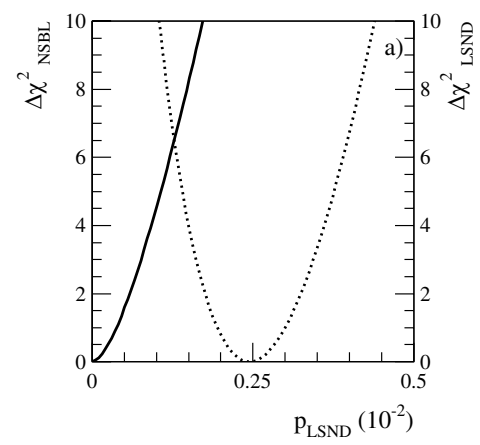

PHYSICAL REVIEW D 70073004

while the minimum at $\Delta m^{2} \sim 10-30 \mathrm{eV}^{2}$ is due to CDHS (mostly) and CCFR84 data, preferring $U_{\mu 4} \neq 0$ values. The $\chi^{2}$ value for no-oscillations, $\left(\chi_{\mathrm{NSBL}}^{2}\right)_{\text {noosc }}=$ 132.2, is the largest $\chi^{2}$ value in Fig. 2(c); this means that the choice of parameters $U_{e 4}=U_{\mu 4}=0$ provides the best-fit to NSBL data, for the $\Delta m^{2}$ values satisfying the condition $\left(\chi_{\mathrm{NSBL}}^{2}\right)_{\min }=\left(\chi_{\mathrm{NSBL}}^{2}\right)_{\text {noosc }}$. Note that the $\Delta m^{2} \simeq$ $0.9 \mathrm{eV}^{2}, \Delta m^{2} \simeq 10-30 \mathrm{eV}^{2}$ dips in $\chi_{\mathrm{NSBL}}^{2}$ are consistent with $U_{e 4} U_{\mu 4}=0$, and therefore with $\sin ^{2} 2 \theta_{\mu e}=$ $p_{\text {LSND }}=0$, but give better fits than the no-oscillations hypothesis, $U_{e 4}=U_{\mu 4}=0$. In other words, the goodness of fit for the $\sin ^{2} 2 \theta_{\mu e}=p_{\mathrm{LSND}}=0$ region depends on the $\Delta m^{2}$ value considered.

Similarly, Fig. 2(d) shows the values for $\left(\chi_{\text {LSND }}^{2}\right)_{\min }$ as a function of $\Delta m^{2}$, used to obtain the LSND allowed regions drawn in Figs. 2(a) and 2(b).

We now present a slightly different approach to determine the statistical compatibility between the NSBL and LSND datasets in $(3+1)$ models, which will prove useful in comparing the $(3+1)$ and $(3+2)$ hypotheses.

In Fig. 3, we show the values for the $\chi^{2}$ differences $\Delta \chi_{\text {NSBL }}^{2}, \Delta \chi_{\text {LSND }}^{2}$, as well as the corresponding confidence levels $\delta_{\text {NSBL }}, \delta_{\text {LSND }}$, as a function of the LSND oscillation probability. The curves are for the set of $(3+1)$ models with the neutrino mass splitting $\Delta m^{2}$ fixed to the best-fit value obtained in a combined NSBL + LSND analysis (see Sec. III B), $\Delta m^{2}=0.92 e V^{2}$, and mixing matrix elements $U_{\mu 4}, U_{e 4}$ treated as free parameters. The value for $\Delta m^{2}$ is chosen in this way because it represents to a good approximation the value for which one expects the best compatibility between the two datasets, as can also be seen in Fig. 2(b). In Fig. 3(a), we map the $\left(U_{e 4}, U_{\mu 4}\right)$ allowed space into the $\left(p_{\mathrm{LSND}}, \chi_{\mathrm{NSBL}}^{2}\right)$, $\left(p_{\text {LSND }}, \chi_{\text {LSND }}^{2}\right)$ spaces. For any given value of $p_{\text {LSND }}$, the minima for the $\chi_{\mathrm{NSBL}}^{2}$ and $\chi_{\mathrm{LSND}}^{2}$ functions are found

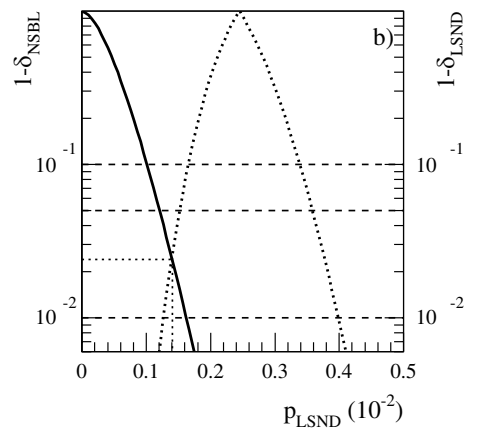

FIG. 3. (a) $\chi^{2}$ differences $\Delta \chi_{\mathrm{NSBL}}^{2}, \Delta \chi_{\mathrm{LSND}}^{2}$, and (b) individual confidence levels $\delta_{\mathrm{NSBL}}, \delta_{\mathrm{LSND}}$, as a function of the LSND oscillation probability $p_{\text {LSND }}$, for the NSBL and LSND datasets. The curves are for $(3+1)$ models with the neutrino mass splitting $\Delta m^{2}$ fixed to the best-fit value $\Delta m^{2}=0.92 \mathrm{eV}^{2}$ from the combined NSBL + LSND analysis, and variable mixing matrix elements $U_{\mu 4}, U_{e 4}$. The solid curves refer to the NSBL dataset, the dotted ones to the LSND dataset. The dashed horizontal lines in Figure (b) refer to the $90 \%, 95 \%, 99 \%$ individual confidence levels, the dotted horizontal line gives the combined confidence level $\delta=$ $\delta_{\mathrm{NSBL}}\left[\delta_{\mathrm{LSND}}+\left(1-\delta_{\mathrm{LSND}}\right) / 2\right]$ for which the NSBL and LSND datasets are incompatible. 
M. SOREL, J. M. CONRAD, AND M. H. SHAEVITZ

in the two $\left(U_{e 4}, U_{\mu 4}\right)$ and one $\left(U_{e 4} U_{\mu 4}\right)$ free parameters available, respectively. The process is repeated for several $p_{\text {LSND }}$ values, and the collection of these minima for all values of $p_{\text {LSND }}$ give the two curves in Fig. 3(a). The individual confidence levels $\delta_{\mathrm{NSBL}}, \delta_{\mathrm{LSND}}$, shown in Fig. 3(b), are obtained from $\Delta \chi_{\mathrm{NSBL}}^{2}, \Delta \chi_{\mathrm{LSND}}^{2}$ in the usual way, by assuming one and two free parameters for the LSND and NSBL datasets, respectively.

We now address how to extract areas in parameter space of a given combined confidence $\delta$ from two independent experimental constraints, in our case obtained via the NSBL and LSND datasets, without assuming statistical compatibility a priori. The most straightforward way (described, for example, in [30,31]) is to assign a confidence level $\delta=\delta_{\mathrm{NSBL}}\left[\delta_{\mathrm{LSND}}+\left(1-\delta_{\mathrm{LSND}}\right) / 2\right]$ to the overlapping part (if any) between the two separate allowed regions in parameter space which are found with the constraint $\delta_{\mathrm{NSBL}}=\delta_{\mathrm{LSND}}$. The extra factor $(1-$ $\left.\delta_{\text {LSND }}\right) / 2$ is due to the fact that the LSND allowed region in the oscillation probability is two-sided, and overlap with the NSBL result on the same probability is obtained only for downward fluctuations in the LSND result, and not for upward ones.

From Fig. 3(b), we find overlapping allowed ranges in $p_{\mathrm{LSND}}$ for $1-\delta_{\mathrm{NSBL}}=1-\delta_{\mathrm{LSND}} \simeq 2.4 \%$. We conclude that, in $(3+1)$ models, the LSND and NSBL datasets are incompatible at a combined confidence of $\delta \simeq 96.4 \%$. In our opinion, this value does not support any conclusive statements against the statistical compatibility between NSBL and LSND data in $(3+1)$ models, although it

\section{PHYSICAL REVIEW D 70073004}

represents poor agreement between the two datasets. The reader should also refer to Sec. V D, where a different method to quantify the compatibility between the NSBL and LSND results is discussed. Future short-baseline constraints on $\nu_{\mu} \rightarrow \nu_{e}$ appearance, as well as on $\nu_{\mu}$ and $\nu_{e}$ disappearance, should be able to definitively establish whether $(3+1)$ models are a viable solution to explain the LSND signal.

\section{B. Combined NSBL + LSND analysis}

The second analysis we perform is a combined NSBL + LSND analysis, with the purpose of obtaining the $(3+1)$ allowed regions in parameter space, in both $\left(\sin ^{2} 2 \theta_{\mu e}, \Delta m^{2}\right)$ and $\left(p_{\text {LSND }}, \Delta m^{2}\right)$ space. A combined analysis of this sort assumes statistically compatible results. In Sec. III A, we have shown that the LSND and NSBL results are marginally compatible, for $(3+1)$ models. In the following, we refer to the $\mathrm{NSBL}+$ LSND dataset as the short-baseline (SBL) dataset, and we construct the $\chi^{2}$ function

$$
\chi_{\mathrm{SBL}}^{2} \equiv \chi_{\mathrm{NSBL}}^{2}+\chi_{\mathrm{LSND}}^{2}
$$

where the two contributions $\chi_{\mathrm{NSBL}}^{2}$ and $\chi_{\mathrm{LSND}}^{2}$ are now simultaneously minimized with respect to the same set of three oscillation parameters $\Delta m^{2}, U_{e 4}, U_{\mu 4}$.

Figures 4(a) and 4(b) show the $90 \%, 95 \%$, and $99 \% \mathrm{CL}$ three-dimensional allowed regions in $\left(\Delta m^{2}, U_{e 4}, U_{\mu 4}\right)$ projected onto the $\left(\sin ^{2} 2 \theta_{\mu e}, \Delta m^{2}\right)$ and $\left(p_{\text {LSND }}, \Delta m^{2}\right)$ two-dimensional regions, respectively, from the com-
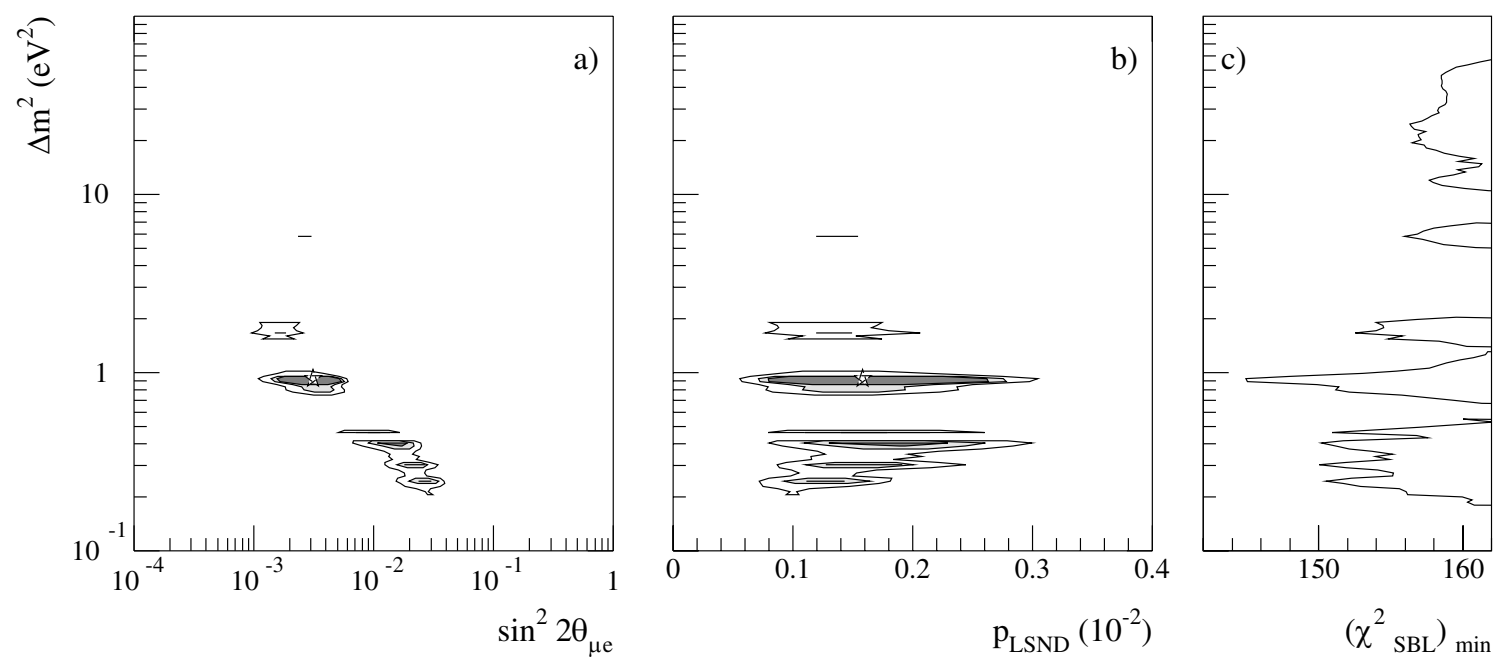

FIG. 4. Allowed regions in parameter space from a combined analysis of NSBL and LSND data, in $(3+1)$ models, assuming statistical compatibility of the NSBL and LSND datasets. Figure (a) shows the 90\%, 95\%, and 99\% CL allowed regions in $\left(\sin ^{2} 2 \theta_{\mu e}, \Delta m^{2}\right)$ space, together with the best-fit point, indicated by the star; (b) shows the same allowed regions in $\left(p_{\text {LSND }}, \Delta m^{2}\right)$ space; (c) shows the minimum $\chi^{2}$ value obtained in the combined analysis as a function of $\Delta m^{2}$. The number of degrees of freedom is 148 . 
COMBINED ANALYSIS OF SHORT-BASELINE...

bined $(3+1)$ analysis of SBL data. In this combined analysis, we use the same Monte Carlo method described in Sec. III A. We define the allowed regions in parameter space by performing a global scan. For example, the $95 \%$ $\mathrm{CL}$ allowed region in the three-dimensional space $\left(\Delta m^{2}, U_{e 4}, U_{\mu 4}\right)$ is obtained by requiring $\chi_{\mathrm{SBL}}^{2}-$ $\left(\chi_{\mathrm{SBL}}^{2}\right)_{\min }<7.82$, where $\left(\chi_{\mathrm{SBL}}^{2}\right)_{\min }$ is the global minimum $\chi^{2}$ value. Figure $4(\mathrm{c})$ shows the minimum $\chi_{\mathrm{SBL}}^{2}$ values obtained in the combined fit, as a function of $\Delta m^{2}$. Of course, the $\chi_{\text {SBL }}^{2}$ values shown in Fig. 4(c) for any given $\Delta m^{2}$ value are larger than the sum of the two contributions $\chi_{\mathrm{NSBL}}^{2}, \chi_{\mathrm{LSND}}^{2}$, shown in Figs. 2(c) and 2(d), for the same $\Delta m^{2}$ value, since the latter were separately minimized with respect to the oscillation parameters. Similarly, Figs. 5(a) and 5(b) show the projections of the $90 \%, 95 \%$, and $99 \% \mathrm{CL}$ allowed regions in $\left(\Delta m^{2}, U_{e 4}, U_{\mu 4}\right)$ onto the $\left(\sin ^{2} 2 \theta_{e e}, \Delta m^{2}\right)$ and $\left(\sin ^{2} 2 \theta_{\mu \mu}, \Delta m^{2}\right)$ space, respectively, from the combined $(3+1)$ analysis of SBL data. The zenith angle distribution of atmospheric muon neutrinos provides a constraint to $\sin ^{2} \theta_{\mu \mu}$ that is not included in this SBL analysis; mixings to the right of the dashed vertical line in Fig. 5(b) are excluded at $90 \%$ CL by atmospheric neutrino results [27]. The global $\chi^{2}$ minimum is $\chi_{\text {SBL }}^{2}=144.9$ (148 d.o.f.). This $\chi^{2}$ value indicates an acceptable fit, assuming that the goodness-of-fit statistic follows the standard $\chi^{2}$ p.d.f. [25]; for an alternative goodness-of-fit test, the reader should refer to Sec. VD. The individual NSBL and LSND contributions to the $\chi^{2}$ minimum are $\chi_{\mathrm{NSBL}}^{2}=137.3$ and $\chi_{\mathrm{LSND}}^{2}=7.6$, respectively. This bestfit point corresponds to the mass and mixing parameters $\Delta m^{2}=0.92 \mathrm{eV}^{2}, U_{e 4}=0.136, U_{\mu 4}=0.205$.
PHYSICAL REVIEW D 70073004

\section{RESULTS FOR $(3+2)$ MODELS}

\section{A. Statistical compatibility between NSBL and LSND}

Having introduced the relevant oscillation probability formula in Eq. (5), and the statistical estimator $p_{\mathrm{LSND}}$ to compare the NSBL and LSND results in Sec. III A, we can now quantitatively address the statistical compatibility between the NSBL and LSND datasets under the $(3+2)$ hypothesis.

Ideally, we would like to determine the NSBL upper limit for $p_{\text {LSND }}$, for all possible combinations of the mass parameters $\Delta m_{41}^{2}, \Delta m_{51}^{2}$. This entails performing a scan equivalent to the one described in the $(3+1)$ case as a function of $\Delta m_{41}^{2}$, shown in Fig. 2. In practice, the CPUtime requirements to pursue this route were prohibitive.

An easier problem to tackle is to determine the statistical compatibility between the NSBL and LSND datasets only for the $(3+2)$ models with mass splittings $\Delta m_{41}^{2}$, $\Delta m_{51}^{2}$ fixed to their best-fit values, as obtained by the combined NSBL + LSND analysis that we present in Sec. IV B. In Sections III A and III B, we have demonstrated that, at least for $(3+1)$ models, this choice is a good approximation for the best possible statistical compatibility (see Figs. 2 and 4).

In Fig. 6, we show the behavior of the $\chi^{2}$ values $\Delta \chi_{\mathrm{NSBL}}^{2}$ and $\Delta \chi_{\mathrm{LSND}}^{2}$, and of the confidence levels $\delta_{\mathrm{NSBL}}$ and $\delta_{\mathrm{LSND}}$, as a function of $p_{\mathrm{LSND}}$, for the set of $(3+2)$ models satisfying the requirements $\Delta m_{41}^{2}=0.92 \mathrm{eV}^{2}$, $\Delta m_{51}^{2}=22 \mathrm{eV}^{2}$. By analogy with Fig. 3, we map the four-dimensional space $\left(U_{e 4}, U_{\mu 4}, U_{e 5}, U_{\mu 5}\right)$ into the two-dimensional spaces $\left(p_{\mathrm{LSND}}, \chi_{\mathrm{NSBL}}^{2}\right)$ and $\left(p_{\mathrm{LSND}}, \chi_{\mathrm{NSBL}}^{2}\right)$, and we plot the minimum $\chi^{2}$ values
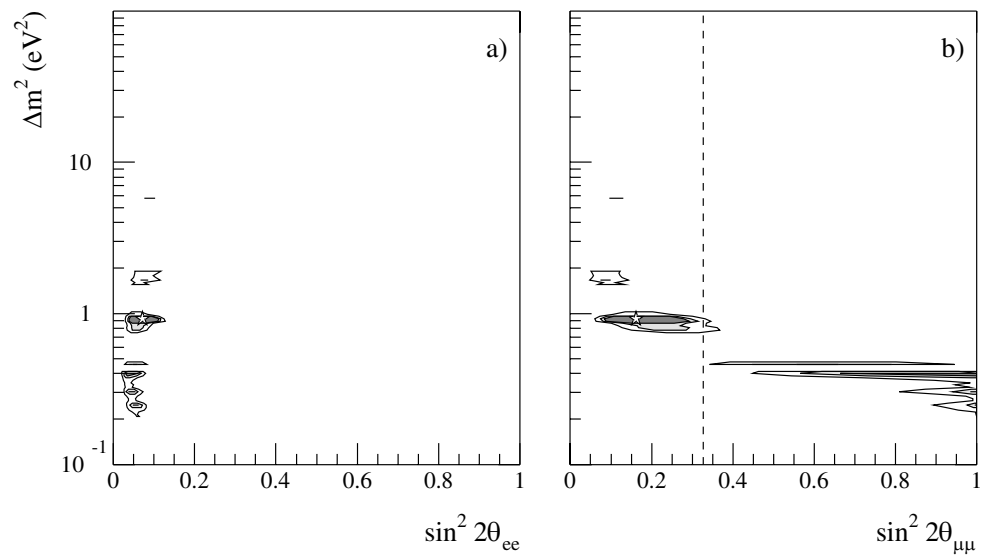

FIG. 5. Allowed regions in the parameter spaces relevant for $\nu_{e}$ and $\nu_{\mu}$ disappearance from a combined analysis of NSBL and LSND data, in $(3+1)$ models, assuming statistical compatibility of the NSBL and LSND datasets. Figure (a) shows the $90 \%, 95 \%$, and 99\% CL allowed regions in $\left(\sin ^{2} 2 \theta_{e e}, \Delta m^{2}\right)$ space, together with the best-fit point, indicated by the star; (b) shows the same allowed regions in $\left(\sin ^{2} 2 \theta_{\mu \mu}, \Delta m^{2}\right)$ space. Mixings to the right of the dashed vertical line in Figure (b) are excluded at $90 \%$ CL by atmospheric neutrino results [27], which are not included in this analysis. 

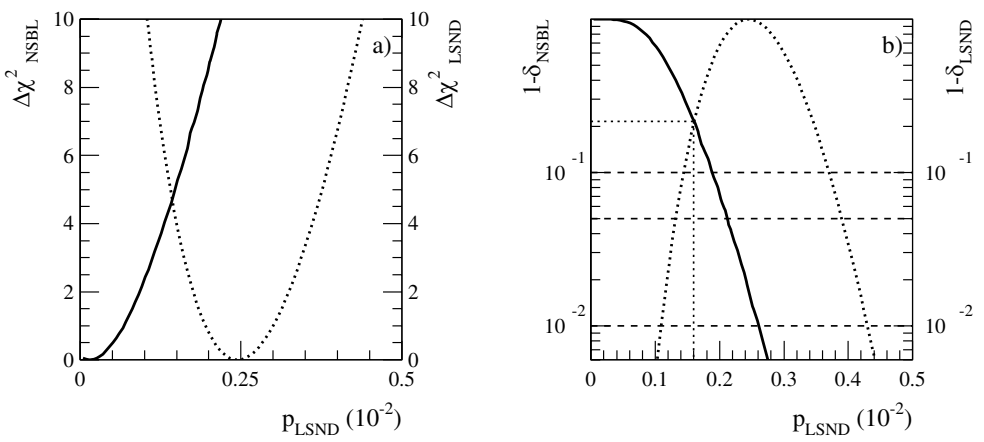

FIG. 6. (a) $\chi^{2}$ differences $\Delta \chi_{\mathrm{NSBL}}^{2}$ and $\Delta \chi_{\mathrm{LSND}}^{2}$, and (b) individual confidence levels $\delta_{\mathrm{NSBL}}$ and $\delta_{\mathrm{LSND}}$, as a function of the LSND oscillation probability $p_{\text {LSND }}$, for the NSBL and LSND datasets. The curves are for $(3+2)$ models with the neutrino mass splittings $\Delta m_{41}^{2}$ and $\Delta m_{51}^{2}$, fixed to the best-fit values $\Delta m_{41}^{2}=0.92 \mathrm{eV}^{2}, \Delta m_{51}^{2}=22 \mathrm{eV}^{2}$ from the combined NSBL + LSND analysis, and variable mixing matrix elements $U_{e 4}, U_{\mu 4}, U_{e 5}, U_{\mu 5}$. The solid curves refer to the NSBL dataset, the dotted ones to the LSND dataset. The dashed horizontal lines in Figure (b) refer to the 90\%, 95\%, 99\% individual confidence levels; the dotted horizontal line gives the combined confidence level $\delta=\delta_{\mathrm{NSBL}}\left[\delta_{\mathrm{LSND}}+\left(1-\delta_{\mathrm{LSND}}\right) / 2\right]$ for which the NSBL and LSND datasets are incompatible.

obtained for any given value of $p_{\text {LSND }}$. The confidence levels shown in Fig. 6(b) are obtained from Fig. 6(a) considering the four free parameters $\left(U_{e 4}, U_{\mu 4}, U_{e 5}\right.$, $\left.U_{\mu 5}\right)$ in the $\chi_{\mathrm{NSBL}}^{2}$ minimization process, and the two free parameters $\left(U_{e 4} U_{\mu 4}, U_{e 5} U_{\mu 5}\right)$ for $\chi_{\mathrm{LSND}}^{2}$.

From Fig. 6(b), we find that, in $(3+2)$ models, the NSBL and LSND datasets are incompatible at an individual confidence level of $\delta_{\mathrm{NSBL}}=\delta_{\mathrm{LSND}}=1-0.215=$ $78.5 \%$, and at a combined confidence level $\delta=$ $\delta_{\text {NSBL }}\left[\delta_{\text {LSND }}+\left(1-\delta_{\text {LSND }}\right) / 2\right]=70.0 \%$. Figure 6 should be compared to Fig. 3, obtained for $(3+1)$ models. A detailed comparison of the $(3+1)$ and $(3+2)$ hypotheses is presented in Sec. V.

\section{B. Combined NSBL + LSND analysis}

We now turn to a combined analysis of the NSBL and LSND results in $(3+2)$ models, assuming statistical compatibility between the two datasets. The purpose of this combined analysis is to obtain the allowed regions in the mass parameter space $\left(\Delta m_{41}^{2}, \Delta m_{51}^{2}\right)$, regardless of the simultaneous values for the mixing parameters. Results will be shown for $\Delta m_{51}^{2} \geq \Delta m_{41}^{2}$; the case $\Delta m_{41}^{2}>\Delta m_{51}^{2}$ can be obtained by simply interchanging $\Delta m_{41}^{2}$ with $\Delta m_{51}^{2}$. The $95 \% \mathrm{CL}$ allowed region is defined as the $\left(\Delta m_{41}^{2}, \Delta m_{51}^{2}\right)$ for which $\chi_{\mathrm{SBL}}^{2}-\left(\chi_{\mathrm{SBL}}^{2}\right)_{\min }<5.99$, where $\left(\chi_{\mathrm{SBL}}^{2}\right)_{\min }$ is the absolute $\chi^{2}$ minimum for all $\left(\Delta m_{41}^{2}, \Delta m_{51}^{2}\right)$ values. In the minimization procedure, the mixing matrix elements $U_{e 4}, U_{\mu 4}, U_{e 5}, U_{\mu 5}$, are treated as free parameters.

Figure 7 shows the $90 \%$ and $99 \%$ CL allowed regions in $\left(\Delta m_{41}^{2}, \Delta m_{51}^{2}\right)$ space obtained in the combined $(3+2)$ analysis. In light of the $(3+1)$ analysis shown in previous sections, the result is not surprising, pointing to favored masses in the range $\Delta m_{41}^{2} \simeq 0.9 \mathrm{eV}^{2}, \Delta m_{51}^{2} \simeq$
$10-40 \mathrm{eV}^{2}$, at $90 \% \mathrm{CL}$. At $99 \% \mathrm{CL}$, the allowed region extends considerably, and many other $\left(\Delta m_{41}^{2}, \Delta m_{51}^{2}\right)$ combinations appear. The best-fit model $\left(\chi_{\mathrm{SBL}}^{2}=135.9\right.$, 145 d.o.f.) is described by the following set of parameters: $\Delta m_{41}^{2}=0.92 \mathrm{eV}^{2}, U_{e 4}=0.121, U_{\mu 4}=0.204, \Delta m_{51}^{2}=$

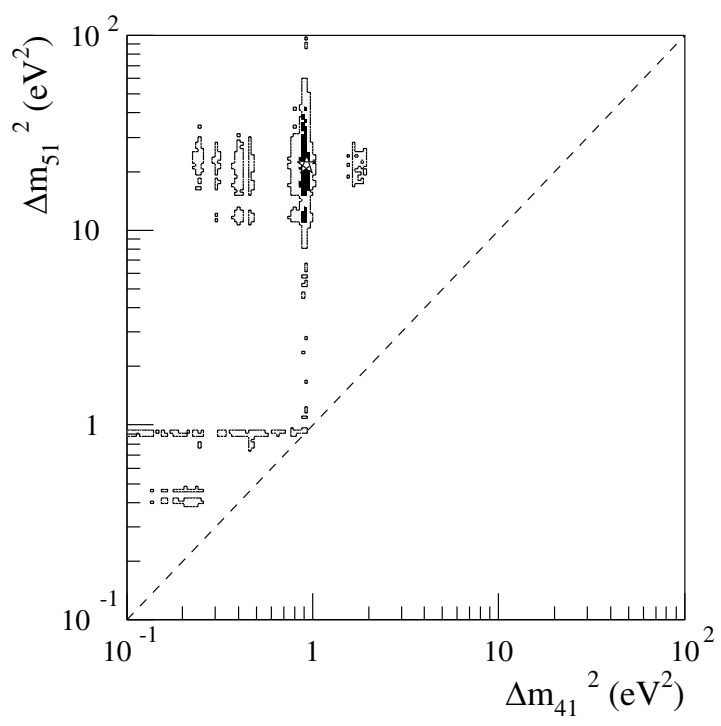

FIG. 7. Allowed ranges in $\left(\Delta m_{41}^{2}, \Delta m_{51}^{2}\right)$ space for $(3+2)$ models, for the combined NSBL + LSND analysis, assuming statistical compatibility between the NSBL and LSND datasets. The star indicates the best-fit point, the dark and light grayshaded regions indicate the 90 and 99\% CL allowed regions, respectively. Only the $\Delta m_{51}^{2}>\Delta m_{41}^{2}$ region is shown; the complementary region $\Delta m_{41}^{2} \geq \Delta m_{51}^{2}$ can be obtained by interchanging $\Delta m_{41}^{2}$ with $\Delta m_{51}^{2}$. 
COMBINED ANALYSIS OF SHORT-BASELINE...

$22 \mathrm{eV}^{2}, U_{e 5}=0.036$, and $U_{\mu 5}=0.224$. We note here that the best fit is not obtained for fourth and fifth mass eigenstates with degenerate masses, that is for $\Delta m_{41}^{2} \simeq$ $\Delta m_{51}^{2}$. The best-fit model we found for sub-eV neutrino masses is $\Delta m_{41}^{2}=0.46 \mathrm{eV}^{2}, U_{e 4}=0.090, U_{\mu 4}=0.226$, $\Delta m_{51}^{2}=0.89 \mathrm{eV}^{2}, \quad U_{e 5}=0.125, \quad U_{\mu 4}=0.160$, corresponding to $\chi_{\mathrm{SBL}}^{2}=141.5$ (145 d.o.f.).

\section{COMPARING THE $(3+1)$ AND $(3+2)$ FITS TO SBL DATA}

In this section, we discuss four statistical tests that can be used to quantify the better overall agreement of SBL data to a $(3+2)$ hypothesis for neutrino oscillations, compared to a $(3+1)$ one.

\section{A. Test 1: NSBL upper limit on $p_{\mathrm{LSND}}$ at a given confidence level $\boldsymbol{\delta}_{\text {NSBL }}$}

Test one uses only NSBL data to establish the $(3+1)$ and $(3+2)$ upper bounds on the LSND oscillation probability $p_{\text {LSND }}$. From Figs. 3 and 6, we obtain at a confidence level $\delta_{\mathrm{NSBL}}=0.90(0.99)$ :

(i) $(3+1): p_{\text {LSND }}<0.100 \%(0.162 \%)$

(ii) $(3+2): p_{\text {LSND }}<0.186 \%(0.262 \%)$

Therefore, we find that $(3+2)$ models can enhance the LSND probability $p_{\text {LSND }}$ by quite a large factor, compared to $(3+1)$ models. The increase in $p_{\text {LSND }}$ that we obtain is significantly larger than the $25 \%$ increase found in [32], which is based on a specific choice of mixing parameters, as opposed to the complete parameter scan performed in this work. The value for the $\bar{\nu}_{\mu} \rightarrow \bar{\nu}_{e}$ oscillation probability measured by LSND [6] is $p_{\mathrm{LSND}}=$ $(0.264 \pm 0.067 \pm 0.045) \%$, where the errors refer to the $1 \sigma$ statistical and systematic errors, respectively.

\section{B. Test 2: statistical compatibility between the NSBL and LSND datasets}

Test two uses both the NSBL and LSND datasets, and treats them independently to find the combined confidence level $\delta=\delta_{\mathrm{NSBL}}\left[\delta_{\mathrm{LSND}}+\left(1-\delta_{\mathrm{LSND}}\right) / 2\right]$ for which the datasets are incompatible, both in $(3+1)$ and $(3+2)$ models. The combined confidence levels can also be read from Figs. 3 and 6 :

(i) $(3+1): \delta=96.4 \%$

(ii) $(3+2): \delta=70.0 \%$

Therefore, we find that in $(3+1)$ models the two datasets are marginally compatible, and the agreement is better in $(3+2)$ models.

\section{Test 3: likelihood ratio test}

Test three combines the NSBL and LSND datasets into a single, joint analysis. The likelihood ratio test [35] provides a standard way to assess whether two hypotheses can be distinguished in a statistically significant way. We define the maximum likelihood $\mathcal{L}_{i}$ from the minimum $\chi^{2}$
PHYSICAL REVIEW D 70073004

values $\left(\chi_{\mathrm{SBL}}^{2}\right)_{\min , i}$ as $\mathcal{L}_{i} \equiv \exp \left[-\left(\chi_{\mathrm{SBL}}^{2}\right)_{\min , i} / 2\right]$, where the index $i=1,2$ refers to the $(3+1)$ and $(3+2)$ hypotheses, respectively. We can then form the likelihood ratio $\lambda_{1,2} \equiv \mathcal{L}_{1} / \mathcal{L}_{2}$. If the $(3+1)$ hypothesis were as adequate as the $(3+2)$ hypothesis in describing SBL data, the quantity

$$
\chi_{1,2}^{2}(3) \equiv-2 \ln \lambda_{1,2}=\left(\chi_{\mathrm{SBL}}^{2}\right)_{\min , 1}-\left(\chi_{\mathrm{SBL}}^{2}\right)_{\min , 2}
$$

should be distributed as a $\chi^{2}$ distribution with three degrees of freedom, where the number of degrees of freedom is the difference in the number of mass and mixing parameters in the $(3+2)$ and $(3+1)$ hypotheses, $6-3=3$

In our combined fits, we obtain (see Sections III B and IV B):

(i) $(3+1):\left(\chi_{\mathrm{SBL}}^{2}\right)_{\min , 1}=144.9,(148$ d.o.f. $)$

(ii) $(3+2)$ : ] $\left(\chi_{S B L}^{2}\right)_{\min , 2}=135.9$, (145 d.o.f. $)$ and therefore $\chi_{1,2}^{2}(3)=9.0$. This value is significantly larger than three; the probability for a $\chi^{2}$ distribution with 3 degrees of freedom to exceed the value 9.0 is only $2.9 \%$. In other words, according to the likelihood ratio test, the $(3+1)$ hypothesis should be rejected compared to the $(3+2)$ one at the $97.1 \% \mathrm{CL}$. Therefore, based on this test, we conclude from test three also that $(3+2)$ models fit SBL data significantly better than $(3+1)$ models.

\section{Test 4: compatibility using the "parameter good- ness of fit"}

Test four uses both the results of the individual NSBL and LSND analyses, as well as the results of the combined NSBL + LSND analysis. The test is based on the "parameter goodness of fit" [36] to compare the compatibility of the NSBL and LSND results under the $(3+1)$ and $(3+2)$ hypotheses. The test avoids the problem that a possible disagreement between the two results is diluted by data points which are insensitive to the mass and mixing parameters that are common to both datasets. The number of parameters common to both datasets is $P_{c}=2$ in $(3+1)$ models, and $P_{c}=4$ in $(3+2)$ models. One possible choice of common parameters is $\left(\Delta m_{41}^{2}, U_{e 4} U_{\mu 4}\right)$ for $(3+1)$ models, $\left(\Delta m_{41}^{2}, U_{e 4} U_{\mu 4}, \Delta m_{51}^{2}, U_{e 5} U_{\mu 5}\right)$ for $(3+2)$ models. The test is based on the statistic $\chi_{\mathrm{PG}}^{2}=\chi_{\mathrm{PG}, \mathrm{NSBL}}^{2}+$ $\chi_{\mathrm{PG}, \mathrm{LSND}}^{2}, \quad$ where $\quad \chi_{\mathrm{PG}, \mathrm{NSBL}}^{2} \equiv\left(\chi_{\mathrm{NSBL}}^{2}\right)_{S B L \mathrm{~min}}-$ $\left(\chi_{\mathrm{NSBL}}^{2}\right)_{N S B L \min } \quad$ and $\quad \chi_{\mathrm{PG}, \mathrm{LSND}}^{2} \equiv\left(\chi_{\mathrm{LSND}}^{2}\right)_{S B L \min }-$ $\left(\chi_{\mathrm{LSND}}^{2}\right)_{L S N D \min }$ are the (positive) differences for the NSBL and LSND $\chi^{2}$ values obtained by minimizing the entire SBL $\chi^{2}$ function, minus the $\chi^{2}$ values that best fit the individual datasets.

Table I gives the values for the parameter goodness of fit PG as defined in [36], based on the $\chi_{\mathrm{PG}}^{2}$ statistic, and the number of parameters common to the NSBL and LSND datasets, $P_{c}$. This test shows a dramatic improve- 
M. SOREL, J. M. CONRAD, AND M. H. SHAEVITZ

TABLE I. Parameter goodness-of-fit PG, as defined in [36], to test the statistical compatibility between the NSBL and LSND datasets under the $(3+1)$ and $(3+2)$ hypotheses. The quantities $\chi_{\mathrm{PG}, \mathrm{NSBL}}^{2}$ and $\chi_{\mathrm{PG}, \mathrm{LSND}}^{2}$ are the NSBL and LSND contributions to the test statistic $\chi_{\mathrm{PG}}^{2}$ defined in the text; $P_{c}$ indicates the number of parameters common to both datasets.

\begin{tabular}{cccccc}
\hline \hline Model & $\chi_{\mathrm{PG}, \mathrm{NSBL}}^{2}$ & $\chi_{\mathrm{PG}, \mathrm{LSND}}^{2}$ & $\chi_{\mathrm{PG}}^{2}$ & $P_{c}$ & $\mathrm{PG}(\%)$ \\
\hline$(3+1)$ & 11.8 & 4.3 & 16.1 & 2 & $3.2 \cdot 10^{-2}$ \\
$(3+2)$ & 7.1 & 4.4 & 11.5 & 4 & 2.1 \\
\hline \hline
\end{tabular}

ment in the compatibility between the NSBL and LSND results in going from a $(3+1)$ to a $(3+2)$ model, raising the compatibility by nearly 2 orders of magnitude, from $0.03 \%$ to $2.1 \%$. It will be interesting to investigate if $(3+3)$ models can improve the compatibility further. The resulting compatibility levels obtained with the parameter goodness-of-fit method are lower than those found in Sec. VB; this, however, is not surprising, since the two statistical tests are quite different.

\section{ADDITIONAL CONSTRAINTS}

The $(3+1)$ and $(3+2)$ models discussed in this work should be confronted with additional experimental constraints, other than the ones discussed in detail in the previous sections. We limit ourselves here to list and comment on some of these constraints, rather than address them in a quantitative way. Mostly, we will discuss the impact that such additional constraints may have on the best-fit $(3+1)$ and $(3+2)$ models found in Sections III and IV.

First, nonzero mixing matrix elements $U_{e 4}, U_{\mu 4}, U_{e 5}$, and $U_{\mu 5}$ may cause observable effects in atmospheric neutrino data, in the form of zenith angle-independent suppressions of the $\nu_{\mu}$ and $\nu_{e}$ survival probabilities. Since our analysis of SBL data tends to give larger values for muon, rather than electron, flavor content in the fourth and fifth mass eigenstate, the effect should be larger on muon atmospheric neutrinos. For example, the $(3+1)$ and $(3+2)$ best-fit models from Sections III B and IV B would give an overall suppression of the $\nu_{\mu}$ flux of $8 \%$ and $17 \%$, respectively. The size of the effect of $\nu_{\mu} \rightarrow \nu_{x}$ oscillations at high $\Delta m^{2}$ is comparable to the current accuracy with which the absolute normalization of the atmospheric neutrino flux is known [37], which is approximately $20 \%$. A more quantitative analysis using the full Super-Kamiokande and MACRO spectral information [27] puts an upper bound of $16 \%$ at $90 \% \mathrm{CL}$ on this high $\Delta m^{2}$ contribution to the atmospheric $\nu_{\mu}$ flux suppression (in the notation of Ref. [27], this suppression is parametrized as $2 d_{\mu}\left(1-d_{\mu}\right)$, where $d_{\mu}<0.09$ at $90 \%$ CL). Therefore, it is expected that the inclusion of atmospheric neutrino data in this analysis would pull the best-fit muon flavor components in the fourth and fifth mass

\section{PHYSICAL REVIEW D 70073004}

eigenstates to lower values, but not in a dramatic way [see also Fig. 5(b)].

Second, models with large masses $m_{4}$ and $m_{5}$, and with nonzero mixing matrix elements $U_{e 4}$ and $U_{e 5}$, should be confronted with tritium $\beta$ decay measurements. The presence of neutrino masses $m_{4}$ and $m_{5}$ introduces kinks in the differential $\beta$ spectrum; the location in energy of the kinks is determined by the neutrino masses, and the size of the kinks is determined by the amount of electron flavor component in the fourth and fifth mass eigenstates. For a spectrometer integrating over the electron energy interval $\delta$ near the $\beta$ decay endpoint, the count rate is [38]

$$
n(\delta)=\frac{\bar{R}}{3} \sum_{i=1}^{n} U_{e i}^{2}\left(\delta^{2}-m_{i}^{2}\right)^{3 / 2},
$$

where the quantity $\bar{R}$ does not depend on the small neutrino masses and mixings, $n=4$ or $n=5$ for $(3+1)$ or $(3+2)$ models, respectively, and we have assumed $\delta>$ $m_{i}, \quad i=1, \ldots, n$, and CP-invariance. From the experimental point of view, tritium $\beta$ decay results are generally expressed in terms of a single effective mass $m\left(\nu_{e}\right)$

$$
n_{s}(\delta)=\frac{\bar{R}}{3}\left[\delta^{2}-m\left(\nu_{e}\right)^{2}\right]^{3 / 2},
$$

where $m\left(\nu_{e}\right)$ is the fit mass parameter. In the limit $\delta^{2} \gg$ $m_{i}^{2}, \quad i=1, \ldots, n$ the relation between the true masses and mixings to the fitted mass $m\left(\nu_{e}\right)$ is independent from the integration interval $\delta$

$$
m\left(\nu_{e}\right)^{2} \simeq \sum_{i=1}^{n} U_{e i}^{2} m_{i}^{2} .
$$

The condition $\delta^{2} \gg m_{i}^{2}, \quad i=1, \ldots, n$ is generally satisfied for the neutrino masses considered in this paper, in order to ensure sufficient $\beta$ decay count rate statistics in the experiments. Therefore, to a first approximation, we can consider the effect of heavy neutrino masses $m_{4}, m_{5}$ only on the single mass parameter $m\left(\nu_{e}\right)$ fitted by the experiments. A more general analysis assessing the sensitivity of current and future $\beta$ decay experiments to multiple fitted neutrino masses, although highly desirable, is beyond the scope of this work; for further details, the reader should consult Ref. [38]. The current best measurements on $m\left(\nu_{e}\right)^{2}$ come from the Troitsk and Mainz experiments [39], which have very similar $m\left(\nu_{e}\right)^{2}$ sensitivities. Both found no evidence for a nonzero $m\left(\nu_{e}\right)^{2}$ value; the latest Mainz result is $m\left(\nu_{e}\right)^{2}=-1.6 \pm$ $2.5 \pm 2.1 \mathrm{eV}^{2}$, or $m\left(\nu_{e}\right) \leq 2.2 \mathrm{eV}$ at $95 \% \mathrm{CL}$, using $\delta=$ $70 \mathrm{eV}$ [39]. Now, assuming a normal hierarchy $\left(m_{1}<\right.$ $m_{4}<m_{5}$ ) with $m_{1} \simeq 0$, the $\beta$ decay neutrino mass in Eq. (12) can be written as $m\left(\nu_{e}\right) \simeq U_{e 4}^{2} \Delta m_{41}^{2}+$ $U_{e 5}^{2} \Delta m_{51}^{2}$; the best-fit $(3+1)$ and $(3+2)$ models found in this analysis would give $m\left(\nu_{e}\right)^{2}=0.017 \mathrm{eV}^{2}$ and $m\left(\nu_{e}\right)^{2}=0.042 \mathrm{eV}^{2}$, respectively, that is $m\left(\nu_{e}\right)^{2}$ values 
COMBINED ANALYSIS OF SHORT-BASELINE...

well below the current experimental sensitivity. The planned tritium $\beta$ decay experiment KATRIN should be able to improve the sensitivity to $m\left(\nu_{e}\right)$ by roughly an order of magnitude in the forthcoming years, thanks to its better statistics, energy resolution, and background rejection [40]. Specifically, the systematic and statistical (for $\delta \gtrsim 30 \mathrm{eV}$ ) uncertainties on the single fitted mass $m\left(\nu_{e}\right)^{2}$ quoted for KATRIN are 0.018 and $0.016 \mathrm{eV}^{2}$, respectively [40], which should provide some sensitivity to the preferred $(3+1)$ and $(3+2)$ neutrino models with a normal mass hierarchy, $m_{1}<m_{4}<m_{5}$. We now consider mass spectra with an inverted hierarchy, defined here as $m_{4}<m_{1}$ for $(3+1)$ models, and $m_{5}<m_{4}<m_{1}$ for $(3+2)$ models. We note that for $(3+2)$ models other hierarchies are also possible, but those do not satisfy the implicit assumption $\left|\Delta m_{51}^{2}\right|=\left|\Delta m_{54}^{2}\right|+\left|\Delta m_{41}^{2}\right|$ taken in this analysis. The $\beta$ decay neutrino mass in Eq. (12) can now be written as $m\left(\nu_{e}\right)^{2} \simeq\left|\Delta m_{41}^{2}\right|$ for inverted $(3+1)$ models, and as $m\left(\nu_{e}\right)^{2} \simeq\left|\Delta m_{51}^{2}\right|$ for inverted(3+ 2) models. Clearly, in this case the $\beta$ decay constraints depend strongly on the values of $\left|\Delta m_{41}^{2}\right|,\left|\Delta m_{51}^{2}\right|$, and models with $\gtrsim 5 \mathrm{eV}^{2}$ mass splittings are already severely disfavored.

Third, introducing sterile neutrinos may affect a number of cosmological predictions, which are derived from various measurements [41]. The standard cosmological model predicts that sterile neutrinos in the $\sim 1 \mathrm{eV}$ mass range with a significant mixing with active neutrinos would be present in the early Universe with the same abundance as the active neutrino species, in disagreement with cosmological observations $[42,43]$. On the other hand, several models have been proposed that would reconcile sterile neutrinos with cosmological observations, for example, suppressing thermal abundances for sterile neutrinos (see, e.g., Ref.[42] and references therein). In particular, active-sterile oscillations in the early Universe may provide a natural mechanism to suppress the relic abundances of sterile neutrinos [44], and scenarios invoking multiple sterile neutrinos are being investigated [43].

\section{CONCLUSIONS}

We have performed a combined analysis of seven shortbaseline experiments (Bugey, CHOOZ, CCFR84, CDHS, KARMEN, LSND, NOMAD) for both the $(3+1)$ and the $(3+2)$ neutrino oscillation hypotheses, involving one and two sterile neutrinos at high $\Delta m^{2}$, respectively. The motivation for considering more than one sterile neutrino arises from the tension in trying to reconcile, in a CPTconserving, four-neutrino picture, the LSND signal for oscillations with the null results obtained by the other short-baseline experiments. Multiple (e.g. three) sterile neutrinos can also be motivated on theoretical grounds.

We have described two types of analyses for both the $(3+1)$ and $(3+2)$ neutrino oscillation hypotheses. In
PHYSICAL REVIEW D 70073004

the first analysis, we treat the LSND and the null shortbaseline (NSBL) datasets separately, and we determine the statistical compatibility between the two. In the second analysis, we assume statistical compatibility and we combine the two datasets, to obtain the favored regions in neutrino mass and mixing parameter space.

The main results of the analysis are summarized in Sec. V, where we compare the adequacy of the $(3+1)$ and $(3+2)$ hypotheses in describing neutrino short-baseline data, by means of four statistical tests. First, we treat the LSND oscillation probability as a parameter that can be measured with NSBL data alone, and find that the NSBL 90\% CL upper limit on the LSND oscillation probability can be significantly relaxed by going from $(3+1)$ to $(3+$ 2) models, by about $80 \%$. Second, the combined confidence level for which the NSBL and LSND datasets are incompatible is determined to be $96.4 \%$ and $70.0 \%$ in the analysis, for the $(3+1)$ and $(3+2)$ hypotheses, respectively. Third, a likelihood ratio test of the two hypotheses is discussed, and shows that the $(3+1)$ hypothesis should be rejected compared to the $(3+2)$ one at the $97.1 \% \mathrm{CL}$. Fourth, the parameter "goodness of fit" defined in [36] shows much better agreement between the NSBL and LSND results for $(3+2)$ models than for $(3+1)$ models.

In conclusion, we find that $(3+1)$ models are only marginally allowed when considering all of the seven short-baseline results, including LSND, in agreement with previous analyses [32-34], and that $(3+2)$ models can provide a better description of the data. Only the simplest neutrino mass and mixing patterns have been fully characterized in the literature so far, and the analysis described in this paper may be viewed as a simple attempt to explore more generic scenarios, which appear both experimentally and theoretically plausible. Given the bright potential for precision measurements by neutrino oscillation experiments in the near future, a more general phenomenological approach may be needed.

\section{ACKNOWLEDGMENTS}

We thank J. Bouchez, L. Camilleri, K. Eitel, J. J. Gomez-Cadenas, E. A. Hawker, G. B. Mills, E. Nagy, V. Valuev, and G. P. Zeller for kindly providing data used in this analysis. We thank K. N. Abazajian, G. Barenboim, S. J. Brice, K. Eitel, B. Kayser, W. C. Louis, M. Maltoni, J. Monroe, P. Nienaber, T. Schwetz, A. Y. Smirnov, and K. Whisnant for valuable discussions and useful suggestions. This work was supported by NSF.

\section{APPENDIX: PHYSICS AND STATISTICAL ASSUMPTIONS}

In this section, we briefly describe the physics and statistical assumptions used to obtain the approximate characterizations of the short-baseline experiments used in the analysis. For the analysis of the Bugey, CDHS, and 
M. SOREL, J. M. CONRAD, AND M. H. SHAEVITZ

KARMEN data, we also refer to the excellent reference [34], which we followed closely.

The Bugey experiment [18] is sensitive to $\bar{\nu}_{e}$ disappearance by measuring the charged-current interaction of $\bar{\nu}_{e}$ 's produced by two nuclear reactors at the Bugey nuclear power plant. Two liquid scintillator detectors, located at different positions, are used. The signature for an antineutrino interaction is a positron and a delayed light pulse produced by the subsequent neutron capture on ${ }^{6} \mathrm{Li}$. Data are given for three baselines: 15, 40, and $95 \mathrm{~m}$ between neutrino production and detection. We follow the "normalized energy spectra" analysis discussed in the Bugey paper [18]. The data are presented as ratios of observed to predicted (for no-oscillations) positron energy spectra, between one and $6 \mathrm{MeV}$ positron energy. We use 25,25 , and ten positron energy bins for the 15, 40, $95 \mathrm{~m}$ baselines, respectively. In the $\chi^{2}$ analysis, fits included not only the mass and mixing parameters, but also five large scale deformations of the positron spectrum due to systematic effects. The experimental positron energy resolution and the neutrino baseline smearing are taken into account; the neutrino cross-section energy dependence within a positron energy bin is not (the energy bin widths are small).

Similarly, the CHOOZ experiment [21] investigates $\bar{\nu}_{e}$ disappearance by observing interactions of $\bar{\nu}_{e}$ 's produced by two nuclear reactors $\simeq 1 \mathrm{~km}$ away from the $\mathrm{CHOOZ}$ detector. The signature for a neutrino interaction is a delayed coincidence between the prompt $e^{+}$signal and the signal due to the neutron capture in the Gd-loaded scintillator. We follow "analysis A," as discussed in the CHOOZ paper [21]. Data are given as positron yields as a function of energy. In this analysis, seven positron energy bins, between 0.8 and $6.4 \mathrm{MeV}$, are considered, for which the CHOOZ observations, as well as the predictions on the positron yields for the no-oscillation case from both reactors, are given in [21]. Because of the presence of two reactor sources, the $\chi^{2}$ analysis comprises 14 positron yield bins for a given energy/baseline. We use the full covariance matrix to take into account the fact that the yields corresponding to the same energy bin are extracted for both reactors simultaneously, as is done in [21]. The analysis fits for the systematic uncertainty in the absolute normalization constant on the $\bar{\nu}_{e}$ yield from the reactors, in addition to the mass and mixing parameters. Since we are interested in the $\Delta m^{2}>0.1 \mathrm{eV}^{2}$ range only, where no energy shape distortions are expected, we neglect the systematic uncertainty on the energy-scale calibration, and the effect of the positron energy resolution.

The CCFR84 experiment [19] constrains $\nu_{\mu}$ and $\bar{\nu}_{\mu}$ disappearance by measuring the charged-current interaction of muon neutrinos and antineutrinos, produced by a Fermilab secondary, sign selected beam yielding $40<$ $E_{\nu}<230 \mathrm{GeV}$ neutrinos from $\pi^{ \pm}$and $K^{ \pm}$decays in the $352 \mathrm{~m}$ long decay pipe. We refer here to the 1984 CCFR

\section{PHYSICAL REVIEW D 70073004}

experiment (hence the label CCFR84 throughout the text), which operated with two similar detectors located at different distances from the neutrino source, 715 and $1116 \mathrm{~m}$ from the midpoint of the decay region, respectively. The two sampling calorimeter detectors consisted of steel plates and scintillation counters. Six secondary beam momentum settings were used, five for neutrino running, and one for antineutrino running. For each secondary beam momentum setting, the data are divided into three neutrino energy bins, for a total of 18 energy bins, from Ref.[45]. Data are presented as double ratios: the far to near detector ratio of observed number of events, divided by the far to near ratio of events predicted for no-oscillations. As in [19], only the mean neutrino energy for a given neutrino energy bin is used in the $\chi^{2}$ analysis. The systematic and statistical uncertainties on the far to near ratio normalization are taken into account. The systematic uncertainty is assumed to be energyindependent and totally correlated between any two energy bins. The neutrino pathlength smearing, mostly due to the long decay region, is also taken into account.

The CDHS experiment [20] is also sensitive to $\nu_{\mu}$ disappearance via the charged-current interaction of $\nu_{\mu}$ 's, produced by a $19.2 \mathrm{GeV} / \mathrm{c}$ proton beam from the CERN Proton Synchrotron. Two detectors are located at 130 and $835 \mathrm{~m}$ from the target. The detectors are sampling calorimeters, with iron and scintillator modules interspersed, to measure the range of a muon produced in a neutrino interaction. Fifteen muon range bins are used. The data are presented as double ratios: the far to near detector ratio of the observed number of events, divided by the far to near ratio of the number of events predicted for no oscillations. Neutrino energy distributions are obtained for a given muon energy (or range) via the NUANCE [46] neutrino cross-section generator. As for CCFR84, the systematic uncertainty on the far to near ratio and the neutrino baseline smearing are taken into account.

The KARMEN experiment [22] investigates the $\bar{\nu}_{\mu} \rightarrow$ $\bar{\nu}_{e}$ appearance channel, from $\bar{\nu}_{\mu}$ 's produced in the $\pi^{+}$ $\mu^{+}$-decay at rest (DAR) chain of the ISIS neutrino source. KARMEN measures the charged-current interaction $p\left(\bar{\nu}_{e}, e^{+}\right) n$, with a liquid scinitillator detector located at an average distance $17.7 \mathrm{~m}$ downstream of the neutrino source. The $\bar{\nu}_{e}$ signature is a spatially correlated delayed coincidence between a prompt positron and a delayed $\gamma$ event from a $(n, \gamma)$ neutron capture reaction. In this analysis, only the positron ("prompt") energy distribution after all cuts is taken into account, given in [22]. The data are binned into nine prompt energy bins, between 16 and $50 \mathrm{MeV}$ (all bins are $4 \mathrm{MeV}$ wide, except the highest energy one, ranging from 48 to $50 \mathrm{MeV}$ ). In predicting the prompt energy distribution for a set of mass and mixing oscillation parameters, the given Monte Carlo positron energy distribution, and the total number of events ex- 
pected after all cuts for full mixing and $\Delta m^{2}=100 \mathrm{eV}^{2}$, are used [47]. Energy resolution and baseline smearing effects (due to finite detector size) are taken into account. Given the low statistics of the nine KARMEN prompt energy bins, we construct the $\chi^{2}$ function by first defining the likelihood ratio [25]

$$
\lambda(\boldsymbol{\theta})=\frac{f[\mathbf{n} ; \boldsymbol{\mu}(\boldsymbol{\theta}), \mathbf{b}]}{f(\mathbf{n} ; \mathbf{n}, \mathbf{b})},
$$

where $\boldsymbol{\theta}$ denotes schematically all mass and mixing parameters, $\mathbf{n}, \boldsymbol{\mu}(\boldsymbol{\theta})$ and $\mathbf{b}$ are the data, expected signal, and expected background vectors with nine elements, and $f[\mathbf{n} ; \boldsymbol{\mu}(\boldsymbol{\theta}), \mathbf{b}]$ are the probabilities for a Poisson process with known background

$$
f[\mathbf{n} ; \boldsymbol{\mu}(\boldsymbol{\theta}), \quad \mathbf{b}]=\prod_{i=1}^{9} \frac{\left(\mu_{i}+b_{i}\right)^{n_{i}} \exp \left[-\left(\mu_{i}+b_{i}\right)\right] .}{n_{i} !}
$$

We define $\chi_{\text {KARMEN }}^{2}$ as

$$
\chi_{\mathrm{KARMEN}}^{2} \equiv-2 \ln \lambda(\boldsymbol{\theta}) .
$$

The LSND experiment at Los Alamos [6] is also sensitive to $\bar{\nu}_{\mu} \rightarrow \bar{\nu}_{e}$ appearance, with a neutrino source and detection signature similar to that of KARMEN, but with better statistics. The LSND liquid scintillator detector is located at an average distance of $30 \mathrm{~m}$ from the neutrino source. As for KARMEN, in this analysis we consider only the positron energy distribution arising from a $\bar{\nu}_{e}$ interaction in mineral oil, published as five energy bins between 20 and $60 \mathrm{MeV}$ [6]. Our analysis ignores the information arising from the higher-energy neutrinos from pions decaying in flight, which has a smaller (but non-negligible) sensitivity to oscillations compared to the decay at rest (DAR) sample considered here. In our simulation, we take into account the expected energy distribution from $\mu^{+}$decay at rest, the neutrino baseline distribution for the $8 \mathrm{~m}$ long cylindrical detector, the neutrino energy dependence of the cross-section for the detection process $p\left(\bar{\nu}_{e}, e^{+}\right) n$ (including nuclear effects, simulated with the NUANCE [46] neutrino cross-section generator), and the experimental energy resolution. We use the published numbers for the background expectations, the number of $\bar{\nu}_{e}$ events for $100 \% \bar{\nu}_{\mu} \rightarrow \bar{\nu}_{e}$ transmutation, and for the efficiency of the event selection criteria. We construct the LSND $\chi^{2}$ function in the same way as we construct the one for KARMEN, because of the low statistics of the data sample.

Finally, the NOMAD experiment is sensitive to $\nu_{\mu} \rightarrow$ $\nu_{e}$ oscillations at $\Delta m^{2} \gtrsim 1 \mathrm{eV}^{2}$ by looking for chargedcurrent muon neutrino and electron neutrino interactions in the NOMAD detector [23]. The detector consists of a large dipole magnet which houses drift chambers to measure the momenta of the charged particles produced in neutrino interactions; transition radiation modules for lepton identification; an electromagnetic calorimeter to measure the energy of electrons and photons; a hadron calorimeter for particle identification; and muon chambers for muon identification. Neutrinos are produced by impinging $450 \mathrm{GeV}$ protons extracted from the CERN SPS accelerator onto a thick beryllium target. The secondary particles produced in the target are focused into a nearly parallel beam by two magnetic lenses, and decay in a $290 \mathrm{~m}$ long decay tunnel to produce a $\sim 10-$ $100 \mathrm{GeV}$ neutrino beam with about $1 \% \nu_{e}$ contamination. Neutrino interactions are then observed in the NOMAD detector at an average distance of $625 \mathrm{~m}$ from the neutrino source. The $\nu_{\mu} \rightarrow \nu_{e}$ search is performed by comparing the measured ratio $R_{e \mu}$ of the number of $\nu_{e}$ to $\nu_{\mu}$ charged-current neutrino interactions with the one expected in the absence of oscillations. The data are binned into 30 bins, covering ten bins in visible energy between three and $170 \mathrm{GeV}$, and three radial bins in the neutrino interaction vertex. A $\chi^{2}$ analysis is performed, using the final NOMAD numbers on the observed and predicted electron-to-muon ratio, including statistical errors as well as the full error matrix describing systematic uncertainties and uncertainty correlations over different bins [48]. In predicting the effect of $\nu_{\mu} \rightarrow \nu_{e}$ oscillations under any mass and mixing hypothesis, the contribution to $R_{e \mu}$ from oscillations with full mixing and $\Delta m^{2}=$ $5000 \mathrm{eV}^{2}$ expected in NOMAD after all cuts are used [48]. Energy resolution and baseline smearing effects (due to the long decay region) are taken into account [23].

In Fig. 8, we show our calculations of the $90 \% \mathrm{CL}$ upper limits on oscillations as a function of $\Delta m^{2}$ for the six NSBL experiments considered here, as well as the 90\% CL allowed region for LSND. The $\left(\chi^{2}\right)_{\min }$ values as a function of $\Delta m^{2}$ for all of the experiments are also shown. All the solid curves shown are obtained from the simplified analysis described here, and compare well with the published results $[6,18-23]$.

The LSND region obtained in our analysis of DAR neutrinos is slightly shifted to the right compared to the final LSND area, shown in Fig. 8(f) as a dashed line, reflecting the difference in the two datasets. More detailed LSND DAR analyses give results in rough agreement with our allowed region $[12,30]$.

The $\left(\chi^{2}\right)_{\min }$ values obtained for the Bugey and CDHS experiments as a function of $\Delta m^{2}$ give details that might seem surprising, at first. Slightly better fits to the data are obtained under a neutrino oscillations hypothesis, as opposed to the no-oscillations one. Therefore, we add a final comment to explain the results of these fits.

The Bugey fit is driven by the data at the shortest baseline, $15 \mathrm{~m}$, where the statistical errors on the observed positron spectrum from $\bar{\nu}_{e}$ interactions are the smallest. As explained in Ref.[18], systematic uncertainties are taken into account by allowing for linear defor- 
M. SOREL, J. M. CONRAD, AND M. H. SHAEVITZ
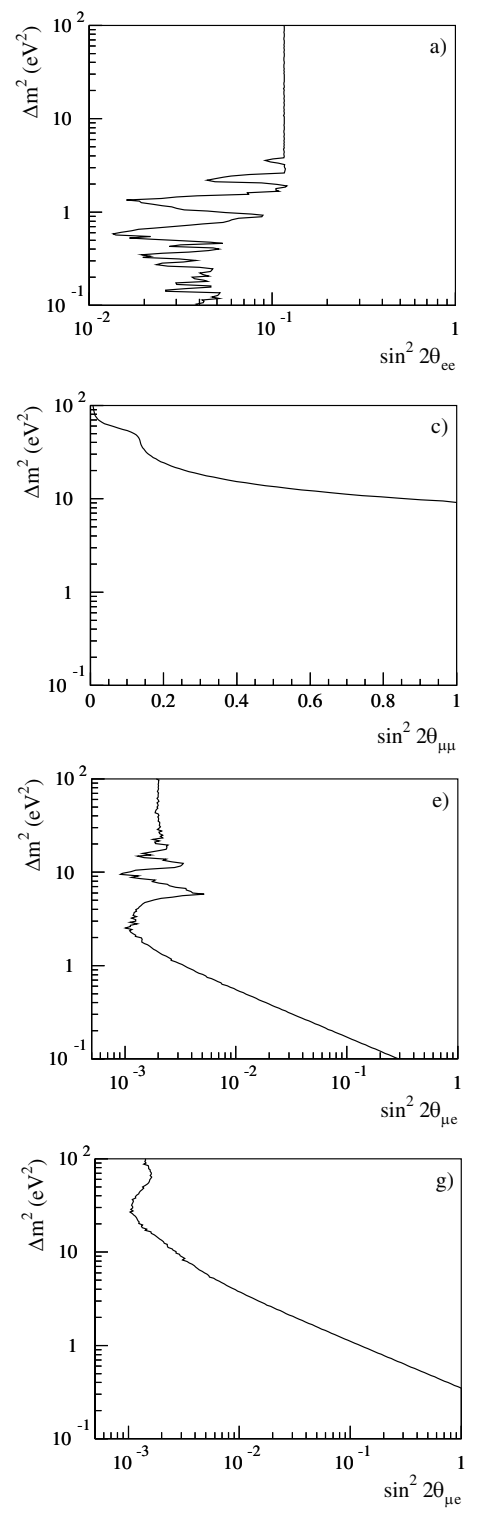
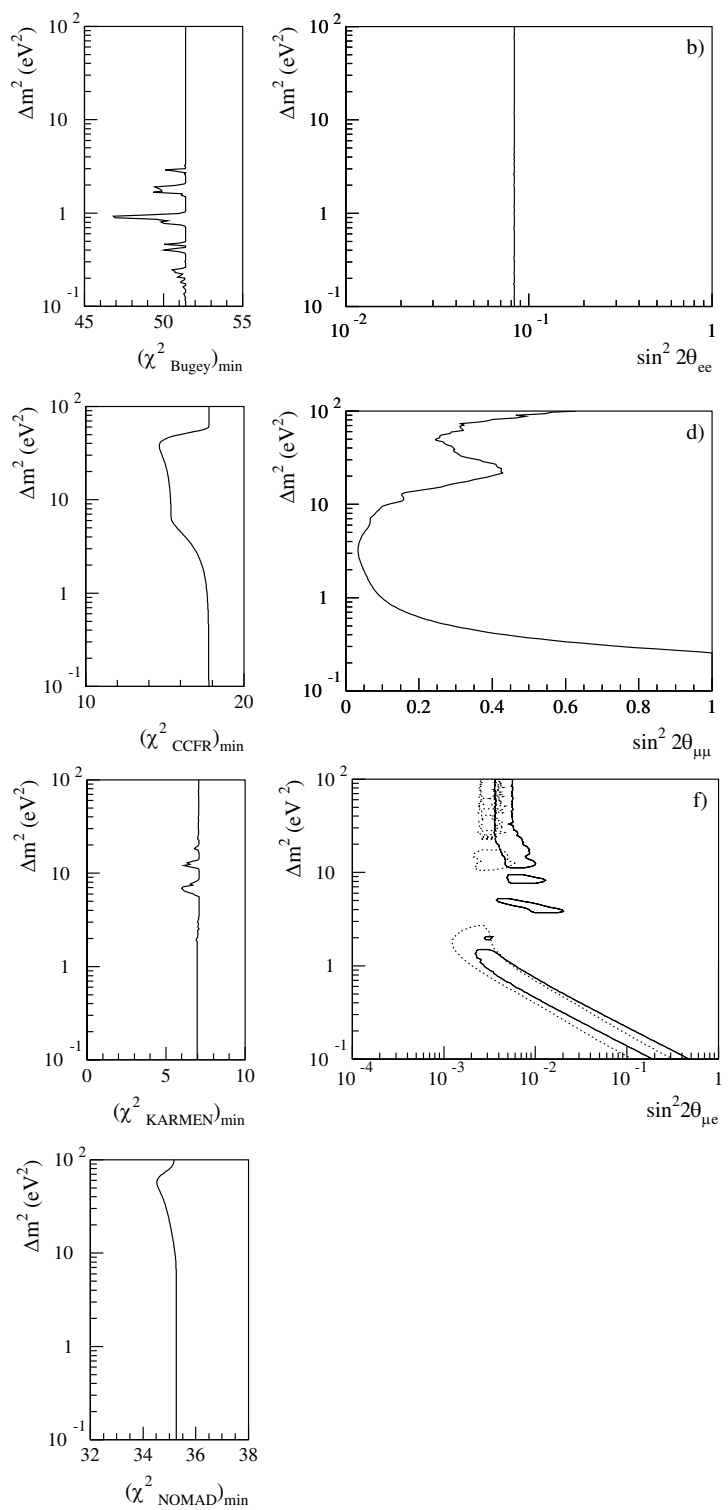

PHYSICAL REVIEW D 70073004
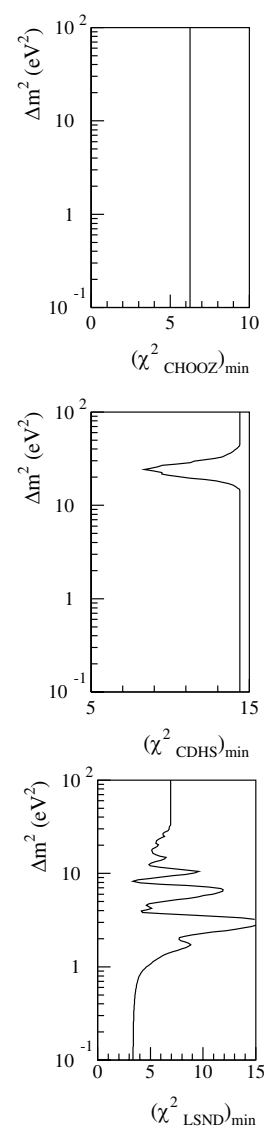

FIG. 8. 90\% CL upper limits on oscillations derived in this analysis for the following NSBL experiments: (a) Bugey, (b) CHOOZ, (c) CCFR84, (d) CDHS, (e) KARMEN, (g) NOMAD. Figure (f) shows the LSND 90\% CL allowed region obtained with the decayat-rest analysis described in the Appendix (solid line), superimposed to the published LSND 90\% CL allowed region (dashed line). Also shown are the $\left(\chi^{2}\right)_{\min }$ values as a function of $\Delta m^{2}$ obtained by all the experiments considered individually. The number of degrees of freedom is 58 in Bugey, 12 in CHOOZ, 16 in CCFR84, 13 in CDHS, seven in KARMEN, three in LSND, 28 in NOMAD.

mations, as a function of positron energy, of the ratio of observed to predicted positron yields. The values of $\left(\chi_{\text {Bugey }}^{2}\right)_{\min }$ as a function of $\Delta m^{2}$ are explained by the fact that, for certain $\Delta m^{2}$ values, an oscillatory fit to the $15 \mathrm{~m}$ positron spectrum ratio describes the data marginally better than any straight line. Our best-fit oscillation hypothesis to Bugey data only is $\Delta m^{2}=0.92 \mathrm{eV}^{2}$, $\sin ^{2} 2 \theta_{e e}=0.05$.

For CDHS, the $\left(\chi_{\text {CDHS }}^{2}\right)_{\text {min }}$ curve in Fig. $8(d)$ has a minimum at $\Delta m^{2} \simeq 20-30 \mathrm{eV}^{2}$. This minimum is due to the fact that the far/near $\nu_{\mu}$ rate ratio, corrected for the baseline and detector mass differences between the two 
COMBINED ANALYSIS OF SHORT-BASELINE...

detectors (as well as other minor effects), is measured to be slightly greater than one [20]: $R_{\text {corr }}=1.044 \pm 0.023 \pm$ 0.025 . This marginal deviation from one causes the fit procedure to prefer more $\nu_{\mu}$ disappearance by oscillations in the near than in the far detector. Given the
PHYSICAL REVIEW D 70073004

average $\nu_{\mu}$ energy $(3.2 \mathrm{GeV})$ and pathlength $(130 \mathrm{~m})$ for neutrinos interacting in the CDHS near detector, this condition is satisfied in the $\Delta m^{2}=20-30 \mathrm{eV}^{2}$ range. Our best-fit oscillation hypothesis to CDHS data only is $\Delta m^{2}=24 \mathrm{eV}^{2}, \sin ^{2} 2 \theta_{\mu \mu}=0.29$.
[1] Kamiokande Collaboration, Y. Fukuda et al., Phys. Lett. B335, 237 (1994); R. Becker-Szendy et al., Nucl. Phys. B, Proc. Suppl. 38, 331 (1995); Soudan-2 Collaboration, W.W. Allison et al. , Phys. Lett. B449, 137 (1999).

[2] Super-Kamiokande Collaboration, Y. Fukuda et al., Phys. Rev. Lett. 81, 1562 (1998).

[3] MACRO Collaboration, M. Ambrosio et al., Phys. Lett. B517, 59 (2001).

[4] B. T. Cleveland et al., Astrophys. J. 496, 505 (1998); GALLEX Collaboration, P. Anselmann et al. Phys. Lett. B285, 376 (1992).D. N. Abdurashitov et al., Phys. Lett. B328, 234 (1994).

[5] SNO Collaboration, Q. R. Ahmad et al., Phys. Rev. Lett. 87, 071301 (2001);SNO Collaboration, Q. R. Ahmad et al., Phys. Rev. Lett. 89, 011301 (2002); SuperKamiokande CollaborationS. Fukuda et al., Phys. Lett. B539, 179 (2002).

[6] LSND Collaboration, C. Athanassopoulos et al. , Phys. Rev. Lett. 77, 3082 (1996);LSND Collaboration, C. Athanassopoulos et al., Phys. Rev. C 58, 2489 (1998);LSND CollaborationA. Aguilar et al., Phys. Rev. D 64, 112007 (2001).

[7] BooNE Collaboration, A. O. Bazarko, hep-ex/9906003.

[8] M. Maltoni, T. Schwetz, and J.W. F. Valle, Phys. Rev. D 65, 093004 (2002).

[9] C. Giunti, M.C. Gonzalez-Garcia and C. Peña-Garay, Phys. Rev. D 62, 013005 (2000).

[10] G. Barenboim and F. Scheck, Phys. Lett. B440, 332 (1998);G. Barenboim, A. Dighe, and S. Skadhauge, Phys. Rev. D 65, 053001 (2002).

[11] H. Murayama and T. Yanagida, Phys. Lett. B520, 263 (2001); G. Barenboim, L. Borissov, J. Lykken, and A.Y. Smirnov, J. High Energy Phys. 0210, 001 (2002);G. Barenboim, L. Borissov, and J. Lykken, hepph/0212116.

[12] M. Maltoni, T. Schwetz, M. A. Tortola, and J.W. F. Valle, Nucl. Phys. B643, 321 (2002).

[13] H. Paes, L. Song, and T. J. Weiler, Phys. Rev. D 67, 073019 (2003).

[14] P. Ramond, hep-ph/9809459.

[15] R. N. Mohapatra and R. E. Marshak, Phys. Rev. Lett. 44, 1316 (1980); ibid.44, 1643(E) (1980)].

[16] R. R. Volkas, Prog. Part. Nucl. Phys. 48, 161 (2002).

[17] G. J. Stephenson, T. Goldman, B. H. J. McKellar, and M. Garbutt, hep-ph/0307245; K. S. Babu and G. Seidl, Phys. Lett. B591, 127 (2004); K. L. McDonald, B. H. J. McKellar, and A. Mastrano, hep-ph/0401241.

[18] Y. Declais et al., Nucl. Phys. B434, 503 (1995).

[19] I. E. Stockdale et al., Phys. Rev. Lett. 52, 1384 (1984).
[20] F. Dydak et al., Phys. Lett. B 134, 281 (1984).

[21] M. Apollonio et al., Eur. Phys. J. C 27, 331 (2003).

[22] KARMEN Collaboration, B. Armbruster et al., Phys. Rev. D 65, 112001 (2002).

[23] NOMAD Collaboration, P. Astier et al., Phys. Lett. B570, 19 (2003); D. Gibin, Nucl. Phys. B, Proc. Suppl. 66, 366 (1998); NOMAD Collaboration, V. Valuev, in International Europhysics Conference on High-Energy Physics (JHEP), Budapest, 2001 [J. High Energy Phys. Conf. Proc., PRHEP-hep2001/190 (2001)].

[24] B. Kayser, hep-ph/0211134.

[25] Particle Data Group Collaboration, K. Hagiwara et al., Phys. Rev. D 66, 010001 (2002).

[26] S. M. Bilenky, C. Giunti, W. Grimus, and T. Schwetz, Phys. Rev. D 60, 073007 (1999).

[27] M. Maltoni, T. Schwetz, and J.W. F. Valle, Phys. Lett. B518, 252 (2001).

[28] F. James and M. Roos, Comput. Phys. Commun. 10, 343 (1975).

[29] G. J. Feldman and R. D. Cousins, Phys. Rev. D 57, 3873 (1998).

[30] E. D. Church, K. Eitel, G. B. Mills, and M. Steidl, Phys. Rev. D 66, 013001 (2002).

[31] K. Eitel, New J. Phys. 2, 1 (2000).

[32] O. L. G. Peres and A. Y. Smirnov, Nucl. Phys. B599, 3 (2001).

[33] A. Strumia, Phys. Lett. B539, 91 (2002).

[34] W. Grimus and T. Schwetz, Eur. Phys. J. C 20, 1 (2001).

[35] W. T. Eadie, D. Drijard, F. E. James, M. Roos, B. Sadoulet, Statistical Methods in Experimental Physics (North Holland, Amsterdam, 1971).

[36] M. Maltoni and T. Schwetz, Phys. Rev. D 68, 033020 (2003); M. Maltoni, T. Schwetz, M. A. Tortola, and J.W. F. Valle, Phys. Rev. D 68, 003020 (2003).

[37] M. Honda, T. Kajita, K. Kasahara, and S. Midorikawa, Phys. Rev. D 52, 4985 (1995); Y. Liu, L. Derome, and M. Buenerd, Phys. Rev. D 67, 073022 (2003).

[38] Y. Farzan and A. Y. Smirnov, Phys. Lett. B557, 224 (2003);Y. Farzan, O. L G. Peres, and A. Y. Smirnov, Nucl. Phys. B612, 59 (2001).

[39] V. M. Lobashev et al., Nucl. Phys. B, Proc. Suppl. 91, 280 (2001); J. Bonn et al., Nucl. Phys. B, Proc. Suppl. 91, 273 (2001).

[40] KATRIN Collaboration, A. Osipowicz et al., hep-ex/ 0109033; KATRIN Collaboration, L. Bornschein et al. in Proceeding of the 23rd International Conference on Physics in Collision, Zeuthen, Germany, 2003, eConf C030626, FRAP14 (2003). 
[41] D. N. Spergel et al., Astrophys. J. Suppl. Ser. 148, 175 (2003); W. J. Percival et al., Mon. Not. R. Astron. Soc. 327, 1297 (2001); J. M. O’Meara, D. Tytler, D. Kirkman, N. Suzuki, J. X. Prochaska, D. Lubin, and A. M. Wolfe, Astrophys. J. 552, 718 ( 2001); W. L. Freedman et al., Astrophys. J. 553, 47 (2001)

[42] K. N. Abazajian, Astropart. Phys. 19, 303 (2003).

[43] P. Di Bari, Phys. Rev. D 67, 127301 (2003); P. Di Bari, Phys. Rev. D 65, 043509 (2002).
[44] R. Foot, M. J. Thomson, and R. R. Volkas, Phys. Rev. D 53, 5349 (1996).

[45] I. E. Stockdale, FERMILAB Report No. FERMILABTHESIS-1984-08, 1984 (unpublished).

[46] D. Casper, Nucl. Phys. B, Proc. Suppl. 112, 161 (2002).

[47] KARMEN collaboration, K. Eitel et al., 77, 212 (1999).

[48] V. Valuev, (private communication). 


\subsection{Short-Baseline Neutrino Experiments and Lep- tonic CP-Violation}

One of the most pressing open questions in neutrino physics today is whether or not leptons fail to conserve the fundamental CP symmetry. The consequences of a leptonic CP symmetry violation would be far-reaching and extend beyond the realm of particle physics, possibly being related to the matter-antimatter asymmetry observed in the Universe today.

In the standard paradigm of three active neutrino mixing occurring at the solar and atmospheric oscillation scales only, leptonic CP violation would yield different vacuum oscillation probabilities for neutrinos and antineutrinos that could be observed with accelerator-based neutrino oscillation appearance experiments operating near the atmospheric oscillation maximum. This is because CP-odd terms in the oscillation probability formula would appear from solar/atmospheric interference terms involving the single CP-violating Dirac phase appearing in the neutrino mixing matrix. An ambitious experimental program is currently being planned to try to measure this CP-violating phase [48].

Neutrino models involving active/sterile neutrino mixing at the LSND neutrino mass splitting scale via at least two sterile neutrino states would open the possibility for further manifestations of leptonic CP violation, including ones that could be measurable with neutrino appearance experiments at short baselines also. In the following, short-baseline leptonic CP-violation in $(3+2)$ sterile neutrino models is discussed, based on work done in collaboration with A. Aguilar-Arevalo, V. Barger, J. Conrad, M. Shaevitz, and K. Whisnant [71]. The first main result of this work is that a combined analysis of current short-baseline oscillation results allow for all possible values for the CP-violating phase that could be measurable at short baselines. The second main result is that CP violation at short baselines could significantly alter the expectations for oscillations in MiniBooNE neutrino running mode, based on the 
LSND antineutrino oscillation signal indication. In this scenario, an additional MiniBooNE antineutrino run would be highly desirable. Third, the studies show that the combination of high-statistics MiniBooNE $\nu_{\mu} \rightarrow \nu_{e}$ and $\bar{\nu}_{\mu} \rightarrow \bar{\nu}_{e}$ searches could, under favorable circumstances, lead to the measurement of this short-baseline CP-violating phase.

\subsubsection{Including CP-Violation in the Neutrino Oscillation For- malism}

As discussed in Section 1.3, for $N$ neutrino species, the general neutrino oscillation formula is:

$$
\begin{aligned}
P\left(\nu_{\alpha} \rightarrow \nu_{\beta}\right)=\delta_{\alpha \beta}-4 & \sum_{i>j} \mathcal{R}\left(U_{\alpha i}^{*} U_{\beta i} U_{\alpha j} U_{\beta j}^{*}\right) \sin ^{2} x_{i j}+ \\
& +2 \sum_{i>j} \mathcal{I}\left(U_{\alpha i}^{*} U_{\beta i} U_{\alpha j} U_{\beta j}^{*}\right) \sin 2 x_{i j}
\end{aligned}
$$

where $\alpha, \beta \equiv e, \mu, \tau$, or sterile flavor, $i, j=1, \ldots, N, x_{i j} \equiv 1.27 \Delta m_{i j}^{2} L / E$, the neutrino mass splitting $\Delta m_{i j}^{2} \equiv m_{i}^{2}-m_{j}^{2}$ is in $\mathrm{eV}^{2}$, the neutrino baseline $L$ in $\mathrm{km}$, and the neutrino energy $E$ is in GeV. There are, in general, $(N-1)$ independent mass splittings, $N(N-1) / 2$ independent moduli of parameters in the unitary mixing matrix, and $(N-1)(N-2) / 2$ Dirac CP-violating phases that may be observed in oscillations.

In short-baseline neutrino experiments that are sensitive only to $\nu_{\mu} \rightarrow \nu_{\mu}, \nu_{e} \rightarrow \nu_{\phi}$, and $\nu_{\mu} \rightarrow \nu_{e}$ transitions, the set of observable parameters simplifies considerably. First, oscillations due to atmospheric and solar mass splittings can be neglected in this case, or equivalently one can set $m_{1}=m_{2}=m_{3}$. Second, mixing matrix elements that measure the $\tau$ neutrino flavor fraction of the various neutrino mass eigenstates do not enter in the oscillation probability. In this case, the number of observable parameters restricts to $(N-3)$ independent mass splittings, $2(N-3)$ moduli of mixing matrix parameters, and $(N-3)(N-4) / 2$ CP-violating phases. Therefore, for $(3+2)$ sterile neutrino models depicted in Fig. 2.1, there are two independent mass splittings 


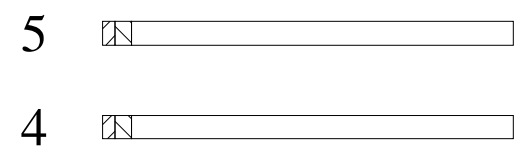

\section{$\Delta \mathrm{m}^{2}$ LSND}

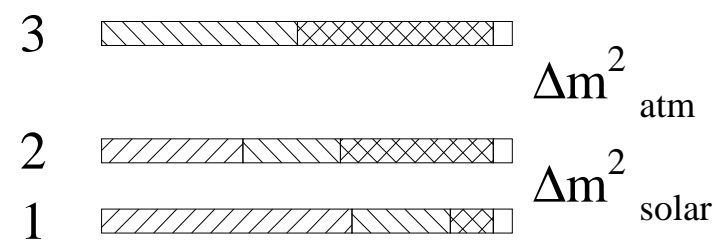

Figure 2.1: Flavor content of neutrino mass eigenstates in (3+2) models. Neutrino masses increase from bottom to top. The $\nu_{e}$ fractions are indicated by right-leaning hatches, the $\nu_{\mu}$ fractions by left-leaning hatches, the $\nu_{\tau}$ fractions by cross-hatches, and the $\nu_{s}$ fractions by no hatches. The flavor contents shown are schematic only.

$\Delta m_{41}^{2}$ and $\Delta m_{51}^{2}$, four moduli of mixing matrix parameters $\left|U_{e 4}\right|,\left|U_{\mu 4}\right|,\left|U_{e 5}\right|,\left|U_{\mu 5}\right|$, and one CP-violating phase. The convention used in the following for this CP-phase is:

$$
\phi_{54}=\arg \left(U_{\mu 5}^{*} U_{e 5} U_{\mu 4} U_{e 4}^{*}\right)
$$

Under these assumptions, the general oscillation formula in Eq. 2.1 can be rewritten as:

$$
\begin{gathered}
P\left(\nu_{\alpha} \rightarrow \nu_{\alpha}\right)=1-4\left[\left(1-\left|U_{\alpha 4}\right|^{2}-\left|U_{\alpha 5}\right|^{2}\right)\left(\left|U_{\alpha 4}\right|^{2} \sin ^{2} x_{41}+\left|U_{\alpha 5}\right|^{2} \sin ^{2} x_{51}\right)+\right. \\
\left.+\left|U_{\alpha 4}\right|^{2}\left|U_{\alpha 5}\right|^{2} \sin ^{2} x_{54}\right] \\
P\left(\nu_{\alpha} \rightarrow \nu_{\beta}\right)=4\left|U_{\alpha 4}\right|^{2}\left|U_{\beta 4}\right|^{2} \sin ^{2} x_{41}+4\left|U_{\alpha 5}\right|^{2}\left|U_{\beta 5}\right|^{2} \sin ^{2} x_{51}+ \\
8\left|U_{\alpha 5}\right|\left|U_{\beta 5}\right|\left|U_{\alpha 4}\right|\left|U_{\beta 4}\right| \sin x_{41} \sin x_{51} \cos \left(x_{54}-\phi_{54}\right)
\end{gathered}
$$

for $\alpha=\beta$ and $\alpha \neq \beta$, respectively. The formulas for antineutrino oscillations are obtained by substituting $\phi_{54} \rightarrow-\phi_{54}$. 


\subsubsection{Analysis Method}

The analysis method used for CP violation studies at short baselines uses similar tools to the ones described in [70], plus a realistic simulation of the MiniBooNE sensitivity to $\stackrel{(-)}{\nu}_{\mu} \rightarrow \stackrel{(-)}{\nu}_{e}$ oscillations, in both neutrino and antineutrino running modes.

\section{Parameter estimation via Monte Carlo techniques}

The analysis uses the same seven short-baseline datasets on $\nu_{\mu}$ disappearance (from the CCFR84 and CDHS experiments), $\nu_{e}$ disappearance (from the Bugey and CHOOZ experiments), and $\nu_{\mu} \rightarrow \nu_{e}$ oscillations (from the LSND, KARMEN2, and NOMAD experiments) as in Ref. [70] to estimate the range of fundamental neutrino parameters in $(3+2)$ models that are experimentally allowed. However, in this case, CP-violating values for the phase $\phi_{54}$ from Eq. 2.2 are also considered, allowing for different neutrino and antineutrino oscillation probabilities in Eq. 2.4.

The Monte Carlo method used in the analysis to estimate the regions in neutrino parameter space that are experimentally allowed by short-baseline results is very similar to the one described in Ref. [70]. Slight modifications to this method have been applied in this case, mainly because of the larger dimensionality of the parameter set present in $\mathrm{CP}$-violating models compared to $\mathrm{CP}$-conserving ones. Rather than generating neutrino masses and mixings in a random, unbiased, way, importance sampling via a Markov chain Monte Carlo method is used [72, 73] , to better sample the regions in parameter space that provide a good fit to short-baseline data. Given a starting point (model) $x_{i}$ in the $\left(\Delta m_{41}^{2},\left|U_{e 4}\right|,\left|U_{\mu 4}\right|, \Delta m_{51}^{2},\left|U_{e 5}\right|,\left|U_{\mu 5}\right|, \phi_{54}\right)$ parameters space, a trial state $x_{i+1}=x_{i}+e$ that depends only on the current state $x_{i}$ and on the probability distribution function for the random vector $e$, is generated. The probability for the trial state $x_{i+1}$ to be accepted as the new current state for further model random generation is given by the transition probability:

$$
P\left(x_{i} \rightarrow x_{i+1}\right)=\min \left\{1, \exp \left[-\left(\chi_{i+1}^{2}-\chi_{i}^{2}\right) / T\right]\right\}
$$


where $\chi_{i}^{2}$ and $\chi_{i+1}^{2}$ are $\chi^{2}$ values for the states $x_{i}$ and $x_{i+1}$, quantifying the agreement between the models and the short-baseline results used in the combined analysis, and $T$ is an effective "temperature" parameter. The results presented here are obtained by combining various Markov chains with different initial conditions, probability distribution functions for $e$, and temperature parameters.

\section{MiniBooNE Sensitivity}

This analysis also provides realistic estimates of the sensitivity to $\stackrel{(-)}{\nu}_{\mu} \rightarrow \stackrel{(-)}{\nu}$ o oscillations that can be achieved with the MiniBooNE experiment, in the framework of $\mathrm{CP}$-conserving and $\mathrm{CP}$-violating $(3+2)$ sterile neutrino models that are currently allowed.

The MiniBooNE sensitivity to $(3+2)$ sterile neutrino oscillations is computed using the full MiniBooNE simulations for neutrino fluxes, neutrino cross-sections, event reconstruction and particle identification in the detector, as done in the MiniBooNE Run Plan [68]. The analysis includes the expected neutrino statistics for $10^{21}$ protons on target in both neutrino and antineutrino running mode, background levels to the $\nu_{e} / \bar{\nu}_{e}$ appearance searches from particle misidentification and intrinsic $\nu_{e}$ 's in the beam, systematic uncertainties associated with those backgrounds, and efficiency in reconstructing and identifying the oscillation signal.

The expected electron neutrino rates for full $\nu_{\mu} \rightarrow \nu_{e}$ transmutation as a function of neutrino energy $E_{\nu}$ are shown in Fig. 2.2, for both neutrino and antineutrino running modes. These distributions are weighted according to the oscillation probability formula in Eq. 2.4 to estimate the number of oscillation signal events for any $(3+2)$ model prior to event reconstruction and particle identification. The sensitivity calculation takes into account the differences in event rates in antineutrino versus neutrino running modes (about a factor of four difference), and the neutrino contribution to the overall event rate in antineutrino mode (a contribution of about $30 \%$ ), which has the effect of washing out CP-violating asymmetries. The MiniBooNE sensitivities 

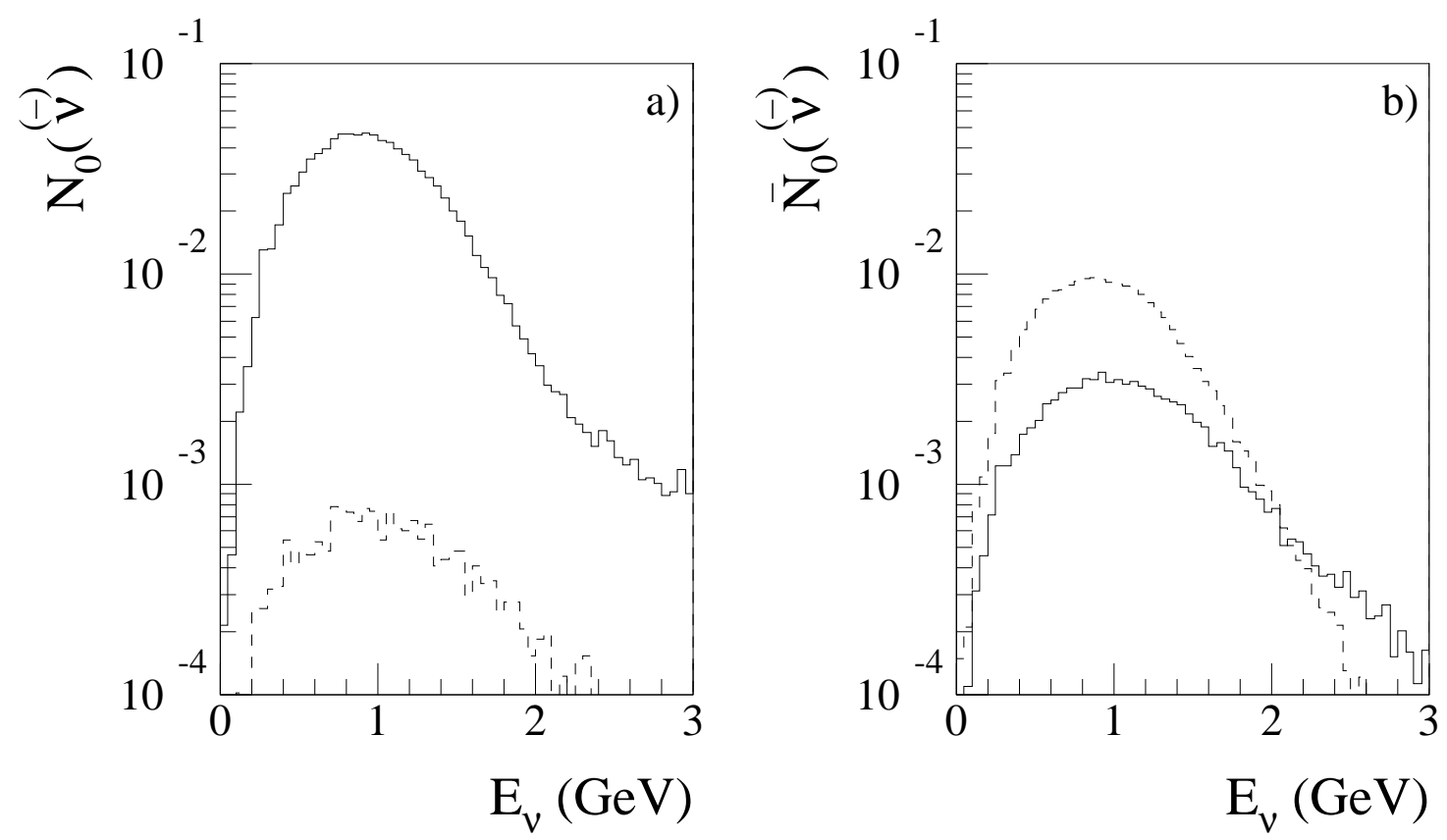

Figure 2.2: Expected MiniBooNE electron neutrino rates for full $\nu_{\mu} \rightarrow \nu_{e}$ transmutation as a function of neutrino energy $E_{\nu}$, in neutrino (panel a)) and antineutrino (panel b)) running modes. The solid histograms are for neutrinos, the dashed ones for antineutrinos. The histograms are normalized such that the overall rate in neutrino running mode is equal to one.

for $10^{21}$ protons on target presented in the following can be considered conservative, to the extent that they assume event counting only, and no fit to the shape of the neutrino energy distribution of the signal is performed.

\subsubsection{Results}

\section{CP-Conserving Case}

Figure 2.3 shows predictions for the oscillation signals to be expected in MiniBooNE neutrino and antineutrino modes, in the CP-conserving, $(3+2)$ sterile neutrino model scenario. The MiniBooNE signal expectations are given in terms of the oscillation 

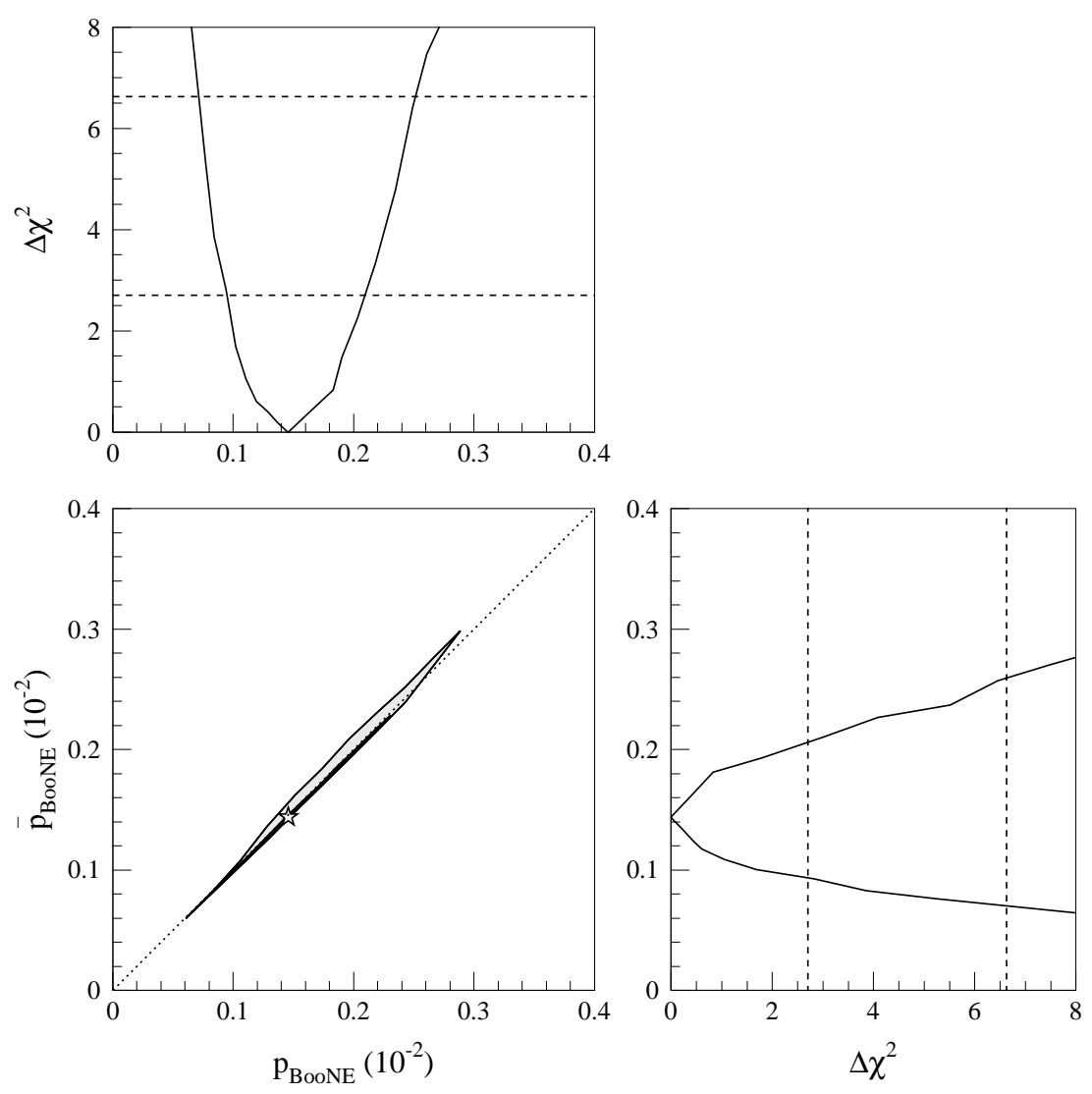

Figure 2.3: Expected oscillation probabilities at MiniBooNE in neutrino and antineutrino running modes, for CP-conserving (3+2) models. See text for details.

probability of Eq. 2.4, weighted by the full transmutation rates:

$$
\stackrel{(-)}{p}_{\text {BooNE }}=\frac{\int d E p\left(\nu_{\mu} \rightarrow \nu_{e}\right)\left[\stackrel{(-)}{N}{ }_{0}(\nu)+\stackrel{(-)}{N}{ }_{0}(\bar{\nu})\right]}{\int d E\left[\stackrel{(-)}{N}{ }_{0}(\nu)+\stackrel{(-)}{N}{ }_{0}(\bar{\nu})\right]}
$$

where $E$ is the neutrino energy, $p\left(\nu_{\mu} \rightarrow \nu_{e}\right)$ is the oscillation probability of Eq. 2.4 in the CP-conserving case $\left(i . e, \phi_{54}=0\right.$ or $\left.\pi\right), N_{0}(\nu)$ and $N_{0}(\bar{\nu})$ are the neutrino and antineutrino full-transmutation rate distributions in neutrino running mode from Fig. $2.2 \mathrm{a}$ ), and $\bar{N}_{0}(\nu)$ and $\bar{N}_{0}(\bar{\nu})$ are the neutrino and antineutrino full-transmutation rate distributions in antineutrino running mode from Fig. 2.2 b).

The bottom left panel in Figure 2.3 shows the region in $\left(p_{\mathrm{BooNE}}, \bar{p}_{\mathrm{BooNE}}\right)$ space that is allowed at the $90 \%$ and $99 \%$ confidence level (2 dof) by existing shortbaseline data used in the analysis, including LSND. The star indicates the best-fit, at 

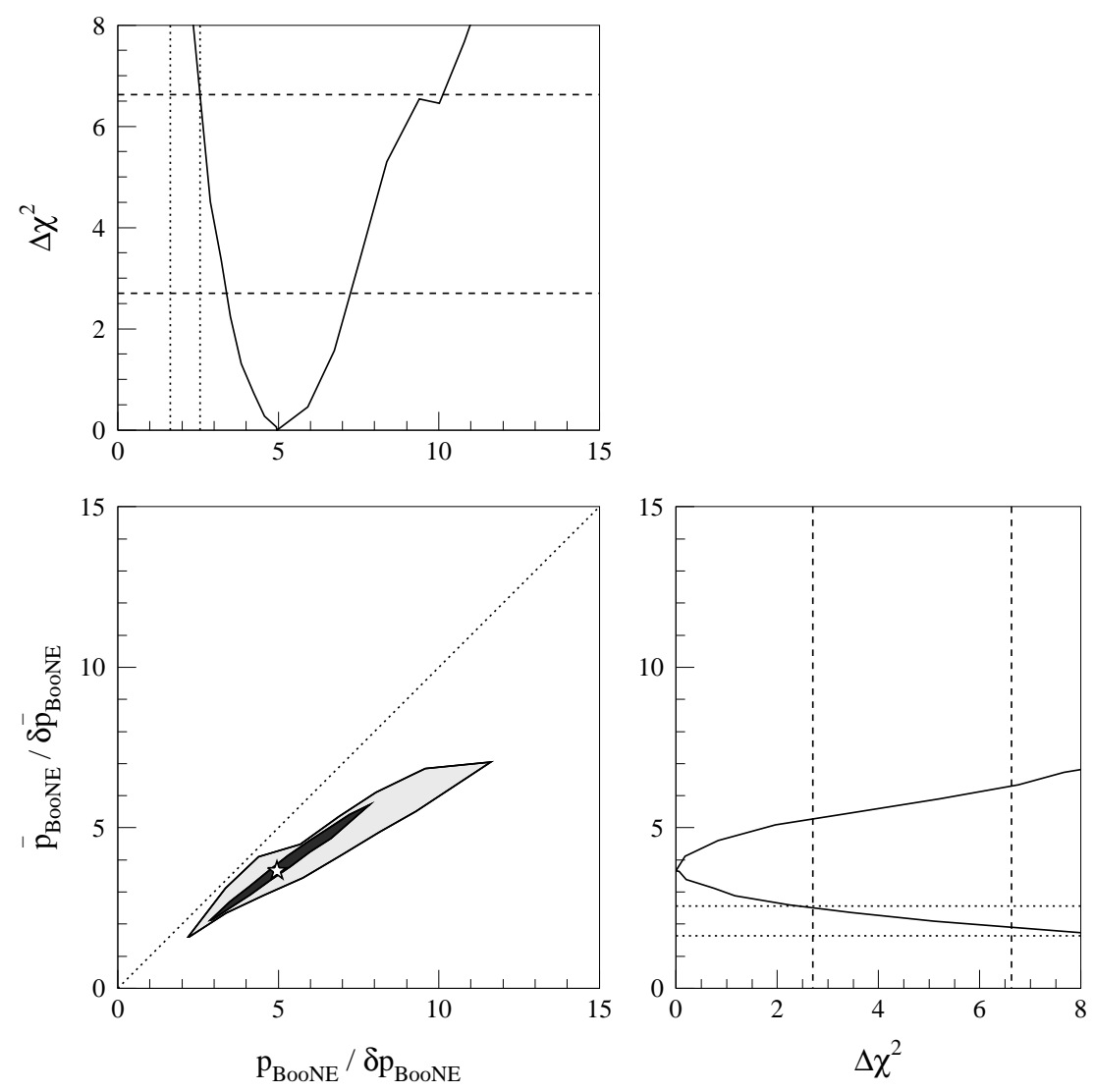

Figure 2.4: Expected oscillation significances at MiniBooNE in neutrino and antineutrino running modes, for CP-conserving (3+2) models. See text for details.

$p_{\mathrm{BooNE}} \simeq \bar{p}_{\mathrm{BooNE}} \simeq 0.14 \cdot 10^{-2}$. The effect of "fake" CP-violation due to spectrum differences in neutrino and antineutrino running modes, which would manifest itself as a departure from the dotted line in the bottom left panel of Fig. 2.3, is at the few percent level at most.

The top left and bottom right panels in Fig. 2.3 show the 1-dimensional projections of the $\chi^{2}$ contours obtained from existing short-baseline data, as a function of $p_{\text {Boone }}$ and $\bar{p}_{\text {BooNE }}$, respectively. The dashed lines at $\Delta \chi^{2}=2.70$ and 6.63 indicate the $90 \%$ and $99 \%$ CL regions, respectively (1 dof). MiniBooNE is expected to measure an oscillation probability in excess of $\simeq 0.07 \cdot 10^{-2}$ in the current neutrino running mode, if CP-conserving $(3+2)$ models are correct.

Figure 2.4 is the same as Fig. 2.3, with the exception that the MiniBooNE 
sensitivities $p_{\mathrm{BooNE}} / \delta p_{\mathrm{BooNE}}$ and $\bar{p}_{\mathrm{BooNE}} / \delta \bar{p}_{\mathrm{BooNE}}$ are shown instead of the oscillation probabilities $p_{\text {BooNe }}$ and $\bar{p}_{\text {BooNE }}$. As mentioned before, the $1 \sigma$ uncertainties $\delta p_{\mathrm{BooNE}}$ and $\delta \bar{p}_{\mathrm{BooNE}}$ associated with the measurement of the oscillation probabilities in neutrino and antineutrino running modes include both the statistical uncertainty of the oscillation signal prediction (for $10^{21}$ protons on target), and the systematic uncertainty of the background prediction.

The bottom left panel in Fig. 2.4 shows that the antineutrino oscillation probability significance is always lower than the neutrino oscillation probability significance, for allowed, CP-conserving $(3+2)$ sterile neutrino models, because of the inherent lower event statistics. Moreover, it is found that the most likely MiniBooNE oscillation significances in an event counting only analysis are about $5 \sigma$ in neutrino mode, and $4 \sigma$ in antineutrino running modes. We note here that these best-fit MiniBooNE oscillation significances are obtained by including several null short-baseline results in the analysis, which tend to reduce the magnitude of the LSND-only neutrino oscillation result.

The top left panel in Fig. 2.4 shows that the MiniBooNE 99\% CL sensitivity ( 1 dof $)$, defined as $p_{\text {BooNE }} / \delta p_{\text {BooNE }} \equiv \sqrt{6.63}=2.57$, just covers all of the neutrino mode oscillation signals from CP-conserving, $(3+2)$ models allowed at 99\% CL (1 dof).

\section{CP-Violating Case}

The same analysis methods and formalism presented above is now applied to CPviolating, $(3+2)$ sterile neutrino models. In this case, the $\mathrm{CP}$-violating phase $\phi_{54}$ can assume any value between 0 and $2 \pi$, and the neutrino and antineutrino oscillation probabilities $p\left(\nu_{\mu} \rightarrow \nu_{e}\right)$ and $p\left(\bar{\nu}_{\mu} \rightarrow \bar{\nu}_{e}\right)$ are generally different. Therefore, the oscillation probabilities in neutrino and antineutrino running modes, averaged over 


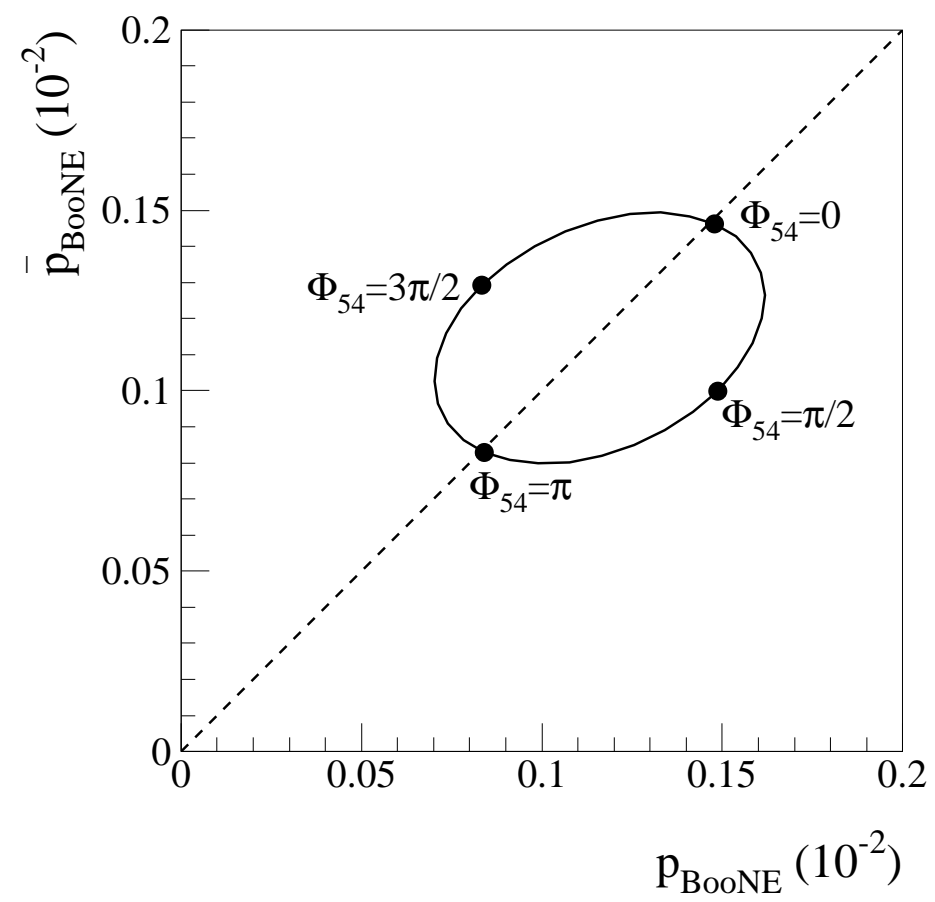

Figure 2.5: Illustration of oscillation probabilities to be expected at MiniBooNE in neutrino and antineutrino running modes, for CP-violating, (3+2) sterile neutrino models. For this particular plot, neutrino masses and mixings are fixed to the values given in the text, while the CP-violating phase $\phi_{54}$ is allowed to vary between 0 and $2 \pi$.

the MiniBooNE spectra, now read:

$$
\stackrel{(-)}{p}_{\text {BooNE }}=\frac{\int d E\left[p\left(\nu_{\mu} \rightarrow \nu_{e}\right) \stackrel{(-)}{N_{0}}(\nu)+p\left(\bar{\nu}_{\mu} \rightarrow \bar{\nu}_{e}\right) \stackrel{(-)}{N_{0}}(\bar{\nu})\right]}{\int d E\left[\stackrel{(-)}{N}_{0}(\nu)+\stackrel{(-)}{N}_{0}(\bar{\nu})\right]}
$$

Figure 2.5 illustrates the order of magnitude of the CP-violating effects to be expected. In this plot, neutrino masses and mixings are fixed to their best-fit values of: $\Delta m_{41}^{2}=0.92 \mathrm{eV}^{2},\left|U_{e 4}\right|=0.123,\left|U_{\mu 4}\right|=0.199, \Delta m_{51}^{2}=21 \mathrm{eV}^{2},\left|U_{e 5}\right|=0.039$, $\left|U_{\mu 5}\right|=0.215$, and the only parameter that is allowed to vary is the CP-violating phase $\phi_{54}$. In this particular case, neutrino/antineutrino running mode oscillation probability differences as big as a factor of two can be obtained, near maximal CPviolation $\left(\phi_{54}=\pi / 2\right.$ or $\left.3 \pi / 2\right)$.

Figure 2.6 shows the oscillation probabilities to be expected at MiniBooNE in neutrino and antineutrino running modes, in a CP-violating, $(3+2)$ scenario. Un- 

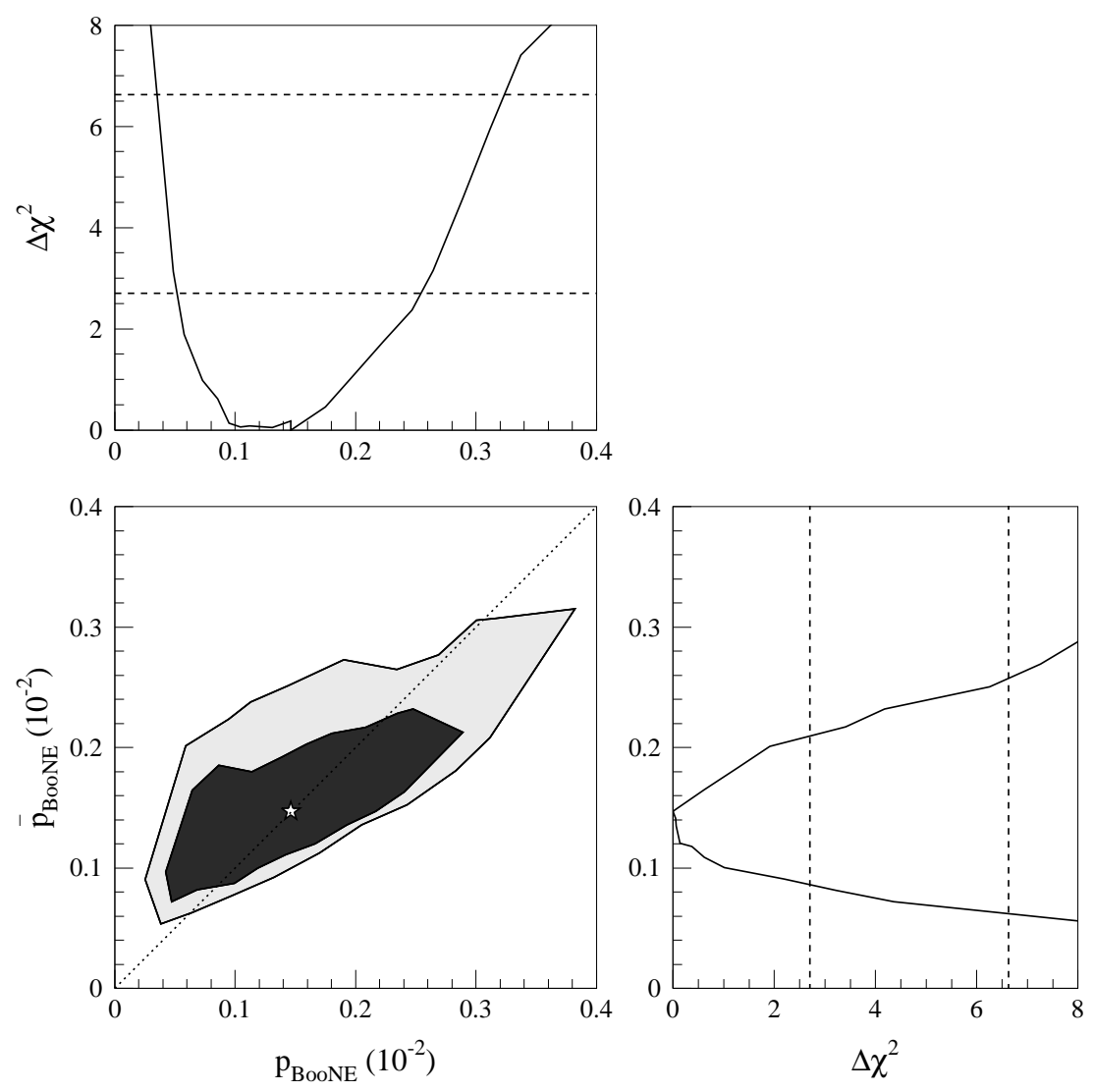

Figure 2.6: Expected oscillation probabilities at MiniBooNE in neutrino and antineutrino running modes, for CP-violating (3+2) models.

like Fig. 2.5, all parameters $\left(\Delta m_{41}^{2},\left|U_{e 4}\right|,\left|U_{\mu 4}\right|, \Delta m_{51}^{2},\left|U_{e 5}\right|,\left|U_{\mu 5}\right|, \phi_{54}\right)$ are now allowed to vary within the constraints provided by existing short-baseline oscillation results. Compared to the $\mathrm{CP}$-conserving case of Fig. 2.3, the best-fit point (indicated by a star) does not change significantly; however, large asymmetries due to CP-violation are now possible, shown by departures from the dashed line in the bottom left panel of Fig. 2.6. The general trend is that the 2-dimensional allowed region in $\left(p_{\mathrm{BooNE}}, \bar{p}_{\mathrm{BooNE}}\right)$ space is tilted more horizontally compared to the dashed line $\bar{p}_{\text {BooNE }}=p_{\text {BooNE }}$, indicating that existing short-baseline results constrain more $\bar{\nu}_{\mu} \rightarrow \bar{\nu}_{e}$ than $\nu_{\mu} \rightarrow \nu_{e}$ oscillations.

In the CP-violating case, MiniBooNE is expected to measure an oscillation probability in excess of $\simeq 0.04 \cdot 10^{-2}$ in the current neutrino running mode. 

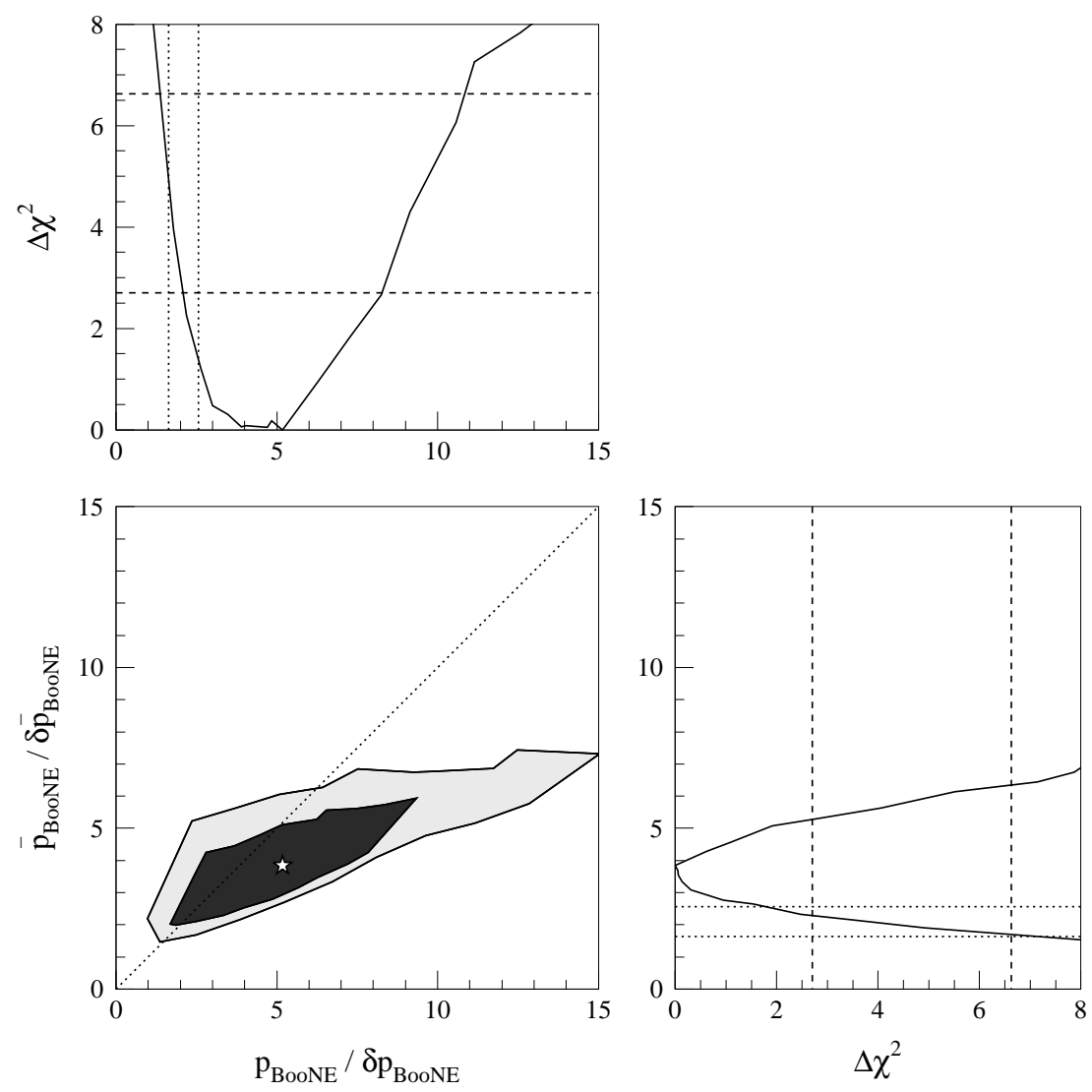

Figure 2.7: Expected oscillation significances at MiniBooNE in neutrino and antineutrino running modes, for CP-violating (3+2) models.

In Fig. 2.7, we show the expected MiniBooNE significances in neutrino and antineutrino running modes, for an oscillation signal in the CP-violating $(3+2)$ scenario. If CP-violation is present, it is possible to have, for certain models, a higher oscillation probability significance in MiniBooNE antineutrino mode rather than in neutrino running mode. In particular, the MiniBooNE 90\% CL sensitivity just covers the $99 \%$ CL (1 dof) allowed oscillation range in antineutrino mode, and a lower coverage is obtained in neutrino mode. Opposite effects are also possible, and significances of more than $10 \sigma$ in neutrino mode could be observed at MiniBooNE in this case.

From this analysis, it is clear that a MiniBooNE antineutrino run would be highly desirable to fully address the LSND antineutrino oscillation signal indication.

Finally, we discuss what are the present constraints on the CP-phase $\phi_{54}$ that 

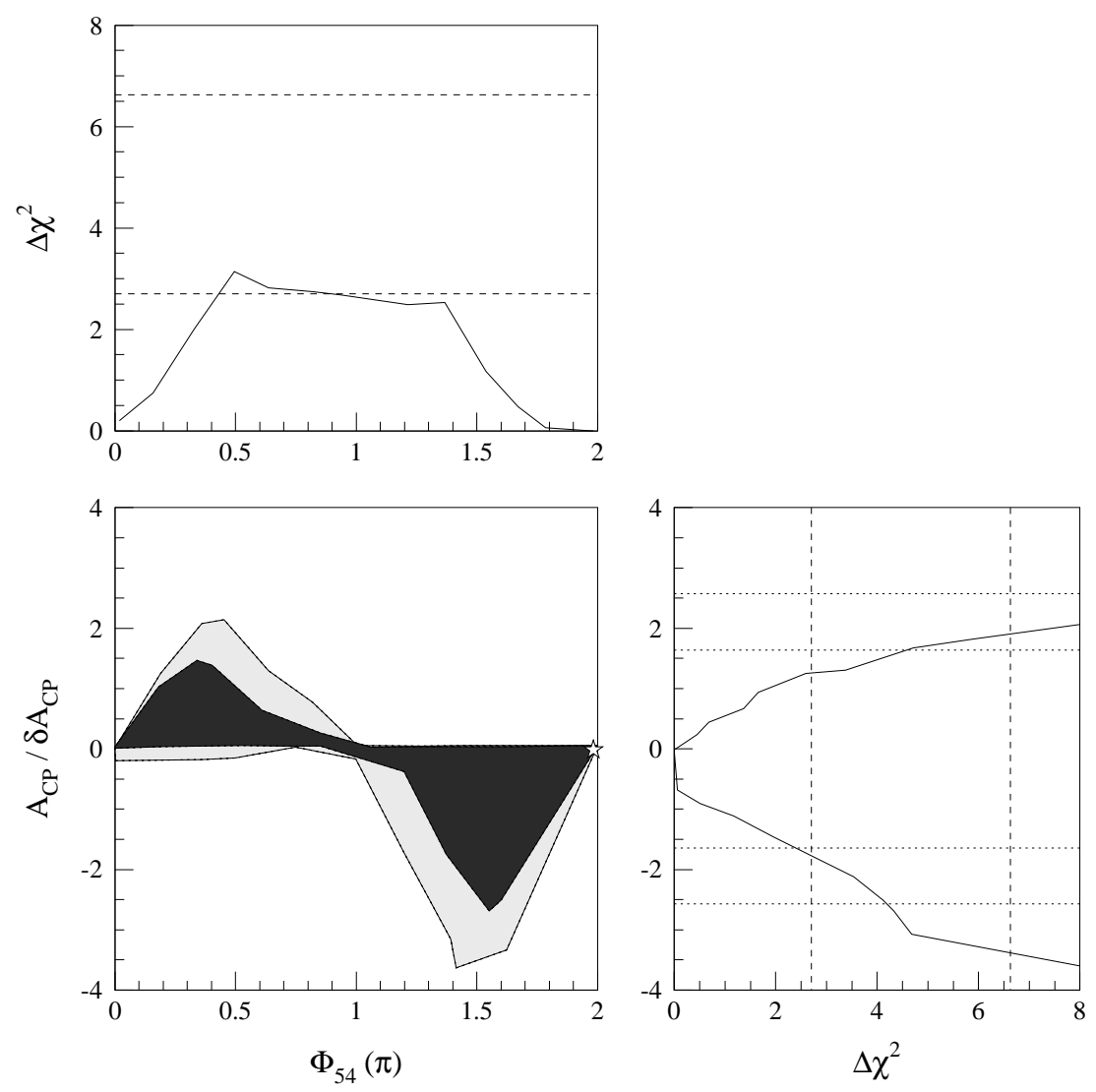

Figure 2.8: Current limits on the CP-violating phase $\phi_{54}$ from current short-baseline results, and significance of a CP asymmetry measurement expected at MiniBooNE, $A_{C P} / \delta A_{C P}$.

are currently available from a CP-violating, $(3+2)$ analysis of short-baseline data, and what is the MiniBooNE CP-asymmetry sensitivity for these allowed values. The CP-asymmetry is defined here as:

$$
A_{C P}=\frac{p_{\mathrm{BooNE}}-\bar{p}_{\mathrm{BooNE}}}{p_{\mathrm{BooNE}}+\bar{p}_{\mathrm{BooNE}}}
$$

The uncertainty on the MiniBooNE CP-asymmetry measurement, $\delta A_{C P}$, is obtained here by standard error propagation, assuming the $\delta p_{\text {BooNE }}$ and $\delta \bar{p}_{\text {BooNE }}$ uncertainties to be uncorrelated. This assumption should be reasonable, to the extent that the MiniBooNE measurements are statistics-dominated, and it represents a conservative assumption.

The top left panel in Fig. 2.8 shows that all values for the CP-phase $\phi_{54}$ are presently allowed at the $99 \%$ confidence level, and that CP-violating, $(3+2)$ models 
with small degrees of CP violation are marginally preferred.

The bottom left plot shows that large CP asymmetry significances are possible (but not required) for maximal CP-violation, given by phases of around $\phi_{54}=\pi / 2$ and $3 \pi / 2$. The dotted lines in the bottom right panel indicate the MiniBooNE $90 \%$ and $99 \%$ CL sensitivity lines to a CP-violating asymmetry, defined as $A_{C P} / \delta A_{C P}=$ $\sqrt{2.70}=1.64$ and $\sqrt{6.63}=2.57$, respectively. The bottom left and right plots indicate that a measurement of CP asymmetry at MiniBooNE with more than $3 \sigma$ significance is possible under favorable circumstances, for CP-violating $(3+2)$ models currently allowed at the $99 \%$ confidence level, even though no significant CP asymmetry would be observed at MiniBooNE in the most likely $(3+2)$ scenarios. 


\section{Chapter 3}

\section{The MiniBooNE Neutrino Beam and the Magnetic Focusing Horn}

In this Chapter, the MiniBooNE neutrino beamline is described. Section 3.1 gives a brief overview of the Fermilab $8 \mathrm{GeV}$ neutrino beamline as a whole. Section 3.2 describes in more detail the MiniBooNE neutrino focusing horn, which has been the hardware project followed during the course of this thesis. Predictions on the neutrino fluxes at the MiniBooNE detector from this beamline are covered in the next Chapter.

\subsection{Overview of the MiniBooNE Neutrino Beam}

The MiniBooNE neutrino beam is a high-intensity, conventional neutrino beam produced via the decay of mesons and muons in a decay region following a target hall region, where meson production and focusing occurs. Mesons are produced in the target hall via inelastic interactions of $8 \mathrm{GeV}$ kinetic energy protons from the Fermilab Booster accelerator in a thick beryllium target, and then focused by a magnetic focusing horn surrounding the target and by a collimator system located downstream of the horn. An overview of the $8 \mathrm{GeV}$ beamline, target hall, decay region and 


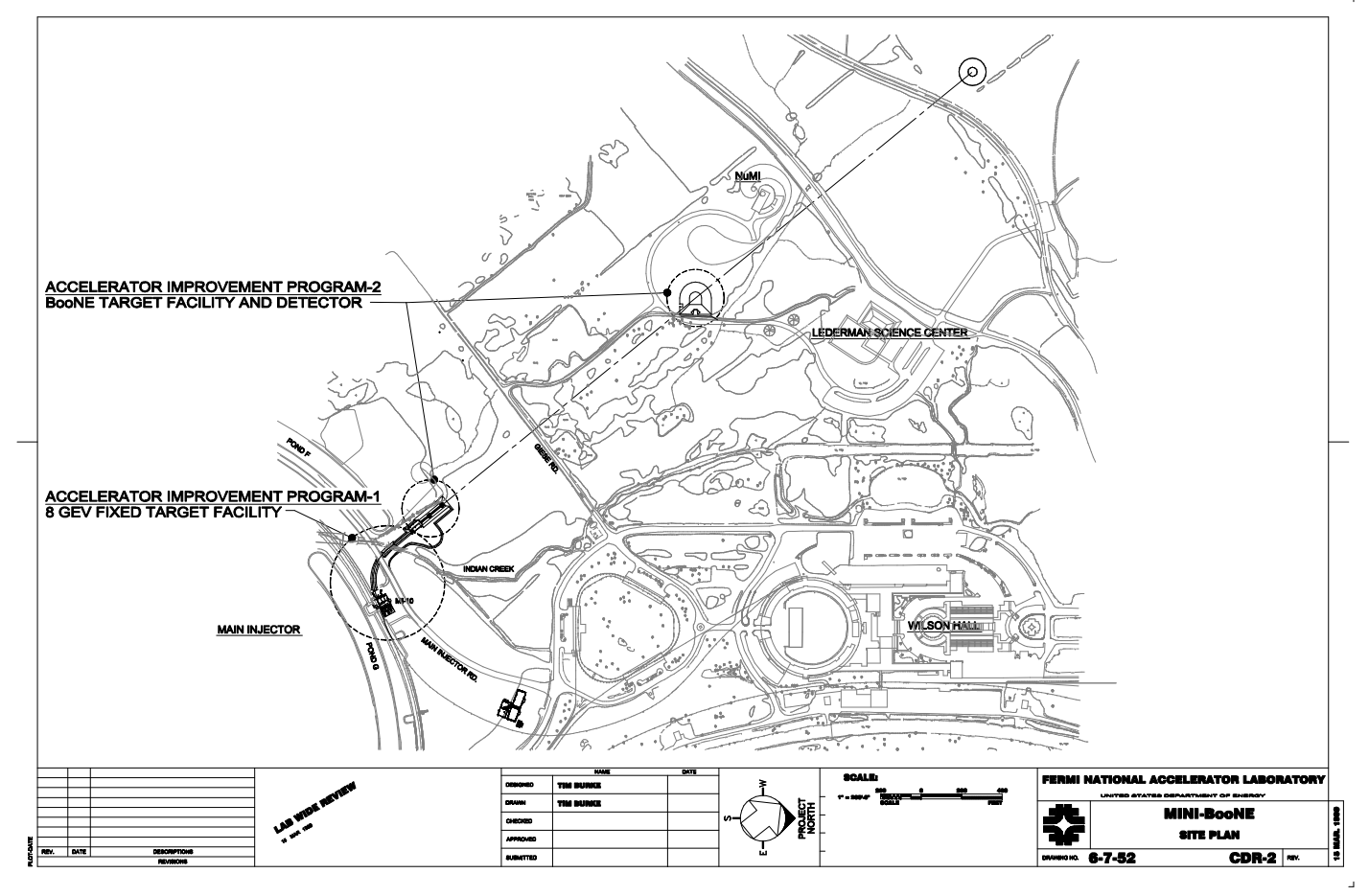

Figure 3.1: Site map of the Fermi National Accelerator Laboratory, showing the location of the 8 GeV neutrino line, the MiniBooNE target hall, decay region, and detector. A possible location for a future second detector is also indicated.

MiniBooNE detector locations is shown in Fig. 3.1.

\subsubsection{The $8 \mathrm{GeV}$ Neutrino Beamline and the Primary Proton Beam}

The primary beam [74, 76] uses protons accelerated to $8 \mathrm{GeV}$ kinetic energy by the Fermilab Booster, and by the earlier stages in the Fermilab proton accelerator complex: the Cockroft-Walton pre-accelerator and the Linear Accelerator. Selected batches containing approximately $5 \cdot 10^{12}$ protons are extracted via a pulsed magnet in the Fermilab Main Injector tunnel, prior to further acceleration by the Main Injector, and bent by about 80 degrees toward the MiniBooNE target hall via dipole magnets. Focusing is provided by permanent and electro-magnet quadrupoles located in the 200 $\mathrm{m}$ long beamline section between the beam extraction location and the MiniBooNE 
target location.

Particle trajectories are monitored on a pulse-by-pulse basis via a system of devices measuring the beam transverse position, direction and profile at various longitudinal positions along the beamline. An automatic beamline correction program uses the measured beam positions to correct in real-time minor deviations from the nominal beam trajectory, by adjusting the magnet settings. The typical beam alignment and divergence measured by the beam position monitors located near the target are within $1 \mathrm{~mm}$ and $1 \mathrm{mrad}$ of the nominal target center and axis direction, respectively; the typical beam focusing on target measured by beam profile monitors is of the order of 1-2 mm (RMS) in both the horizontal and vertical directions. These parameters are well within the experiment requirements, dictated by the $5 \mathrm{~mm}$ in radius, $71 \mathrm{~cm}$ long, cylindrical beryllium target.

The number of protons delivered to the MiniBooNE target is measured for each proton batch, using a toroid located near the target along the beamline. The toroid calibration, performed on a pulse-by-pulse basis, provides a $1 \%$ accuracy on the measurement of the number of protons to MiniBooNE.

Protons that are not properly captured by the beamline optics may yield beam losses along the beam transport line, and may generate hazardous radio-activation of beamline elements, air and ground water. Therefore, several monitors measuring localized beam losses are located along the beamline. Moreover, a monitoring system measuring the total beam losses along the beamline is used, based on the difference between the measured beam intensities at the beamline initial and final locations. A beam permit system based on these (and other) inputs is used to promptly inhibit beam to the MiniBooNE target hall if abnormal conditions occur.

The readouts of beam control (e.g., magnet settings) and monitoring systems (e.g., beam intensity, position, and losses) are recorded and time-stamped for each proton batch, and the beam datastream is synchronized and merged with the corresponding MiniBooNE detector datastream. 


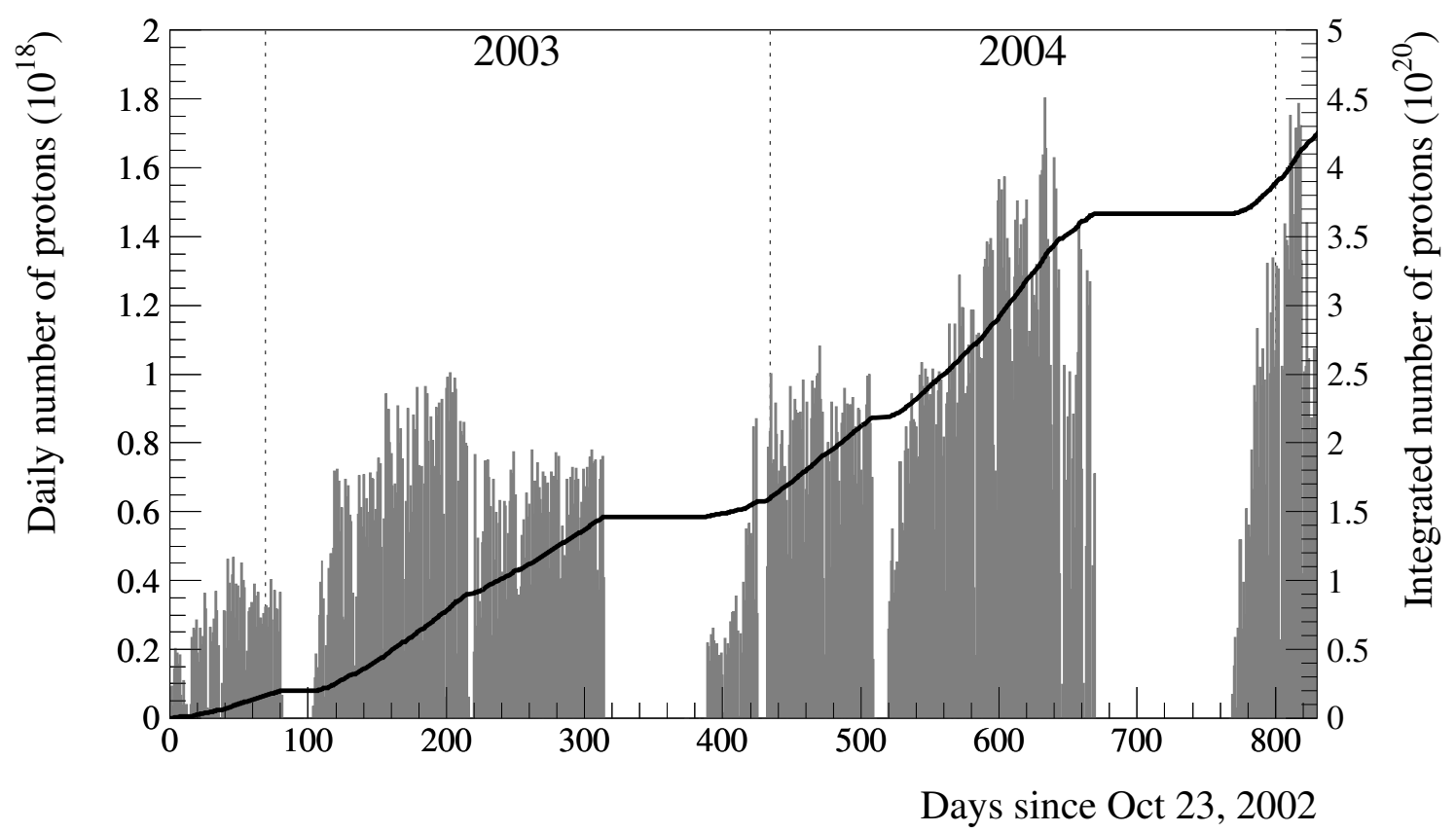

Figure 3.2: Number of protons delivered to the MiniBooNE target in the period between October, 2002, and January, 2005. The histogram shows the daily number of protons, the solid line the integrated number of protons.

The operation of the $8 \mathrm{GeV}$ beamline has been very successful since the September 2002 start-up, with downtimes dominated by scheduled shutdowns of the accelerator complex. Over this period of time, the delivery of protons on the MiniBooNE target has been mostly dictated by the limits imposed on beam losses within the Booster accelerator, and not by losses in the $8 \mathrm{GeV}$ beamline or by hardware limitations. Figure 3.2 shows the number of protons delivered to MiniBooNE since start-up: the positive trend over time in the daily number of protons is indicative of a reduction in the fraction of Booster beam losses. In the period October 2002 - January 2005, more than $4.2 \cdot 10^{20}$ protons have been delivered to MiniBooNE.

\subsubsection{Target Hall and the Secondary Meson Beam}

Primary protons from the $8 \mathrm{GeV}$ beamline strike a thick beryllium target located in the MiniBooNE target hall. Hadronic interactions of the protons with the tar- 
get material produce a beam of secondary mesons (pions and kaons). The target [75] is made of seven cylindrical slugs that are $10.2 \mathrm{~cm}$ in length and $0.48 \mathrm{~cm}$ in radius, corresponding to a total target length of $71 \mathrm{~cm}$, or about 1.7 inelastic interaction lengths. The target geometry is dictated by the requirement of having a large fraction of primary protons that inelastically interact within the target, and a small fraction of reinteractions for the secondary mesons. The multi-slug design is dictated by the requirement of dealing with the thermal shock within the target due to the beam proton pulses. The target material choice, beryllium, is dictated mainly by the requirement of minimizing the radioactivity levels in the target hall due to long-lived isotopes produced by the proton beam exposure.

The target is equipped with a closed air cooling system, in order to dissipate the power $P \simeq 600 \mathrm{~W}$ deposited by the primary and secondary beam in the target under normal operation $\left(5 \cdot 10^{12}\right.$ protons per batch, five batches per second). The timeaveraged beam heat load is efficiently removed by flowing air longitudinally along the target, at a volumetric rate of $d V / d t \simeq 8 \cdot 10^{-3} \mathrm{~m}^{3} / \mathrm{s}$. A redundant system of radiationhard devices monitor the air flow rates and temperatures at various locations in the target cooling system, and inhibit beam if abnormal conditions are measured. Typical measured values for the temperature of the air exiting from the target area are of the order of: $T_{\text {out }}=T_{\text {in }}+P /\left(c_{P} \rho d V / d t\right) \simeq 100 \operatorname{degC}$, where $T_{\text {in }} \simeq 36 \operatorname{degC}$ is the temperature of the air coolant entering the target area, $c_{P}=10^{3} \mathrm{~J} /(\mathrm{kg} \cdot \mathrm{K})$ is the air specific heat at constant pressure, $\rho=1.20 \mathrm{~kg} / \mathrm{m}^{3}$ is the air density, and $P$ and $d V / d t$ are defined above. Target temperatures of the order of $120 \operatorname{deg} \mathrm{C}$ are inferred from the measured air temperature $T_{\text {out }}$, well below the temperatures that may cause permanent damage (softening) to the beryllium and aluminum materials in the target/horn system.

The beryllium target is surrounded by a magnetic focusing horn [75, 77], bending the positively-charged secondary particles that emerge from the interactions in the target along the direction pointing to the MiniBooNE detector. The focusing is 
produced by the toroidal magnetic field present in the air volume between the horn's two coaxial conductors. The magnetic field is produced by current flowing along the horn inner (small radius) conductor, and back along the outer (large radius) conductor. The polarity of the horn current flow can be switched, in order to focus negatively-charged mesons, and therefore produce an anti-neutrino beam instead of a neutrino one. The heat produced in the horn inner conductor by current and by beam deposition is removed via a water cooling system. Details about the physics-driven requirements, mechanical and electrical design, testing, and operational performance for the horn are given in Section 3.2 .

\subsubsection{Decay Region and the Tertiary Neutrino Beam}

The beam of focused, secondary mesons emerging from the target/horn region is further collimated via passive shielding, and allowed to decay into neutrinos in a cylindrical decay region filled with air, $50 \mathrm{~m}$ long and $90 \mathrm{~cm}$ in radius [75]. A beam absorber located at the end of the decay region stops the hadronic and muonic component of the beam, and only a pure neutrino beam pointing toward the detector remains, mostly from $\pi^{+} \rightarrow \mu^{+} \nu_{\mu}$ decays. Details about the meson and muon decays occurring in the decay region that are of relevance for the MiniBooNE neutrino flux are described in Chapter 4 .

The beamline design allows to reduce the length of the decay region to a half of its current length, by lowering an intermediate, movable absorber currently located above the decay pipe, at a distance of $25 \mathrm{~m}$ from the target.

The decay region is also instrumented with a detector capable of measuring the momentum, energy, and charge sign of muons from the decays of kaons in the secondary beam. The muon counter consists of a muon drift pipe, subtending a $7^{\circ}$ angle with respect to the axis of the decay region and intersecting it $9 \mathrm{~m}$ upstream of the $50 \mathrm{~m}$ absorber, and of a collimator, spectrometer and range stack detector located 
near the $50 \mathrm{~m}$ absorber. The kinematic constraints imposed on the muons by this geometrical setup allows the selection of the higher transverse momentum $\left(p_{t}\right)$ muons produced in kaon decays, distinguishing them from the lower $p_{t}$ muons from pion decays, and therefore to constrain the kaon content in the secondary beam.

\subsection{The MiniBooNE Neutrino Focusing Horn}

In the following, the MiniBooNE horn is described in greater detail. The horn physicsdriven requirements and design, horn testing, and horn performance are discussed.

\subsubsection{Horn Physics-Driven Requirements and Design}

The MiniBooNE horn design, and more generally the beamline design, are driven by the requirements for the MiniBooNE $\nu_{\mu} \rightarrow \nu_{e}$ search in the $\Delta m^{2} \simeq 1 \mathrm{eV}^{2}$ range, discussed in Chapter 2. In order to maximize the $\nu_{\mu} \rightarrow \nu_{e}$ sensitivity in the $\Delta m^{2} \simeq$ $1 \mathrm{eV}^{2}$ range (as suggested by the LSND result), the beamline is designed to: a) provide $10^{21}$ protons on target in a timely manner, b) maximize the $\nu_{\mu}$ flux at the detector between 0.3 and $1.3 \mathrm{GeV}$, c) minimize the intrinsic $\nu_{e}$ flux contamination in the beam, d) be operated in both neutrino and antineutrino running modes, and e) understand well the neutrino flux systematics.

The MiniBooNE horn was designed with these physics goals in mind. First, most of the design criteria for the two MiniBooNE horns built so far were dictated by the necessity to withstand an average (instantaneous) pulse repetition rate of 5 (15) $\mathrm{Hz}$ over a lifetime of approximately 100 million pulses at $170 \mathrm{kA}$ peak horn current, to meet the proton delivery requirements (goal a)). Second, the horn geometrical shape and horn current specifications are essential factors in meeting the $\nu_{\mu}$ flux optimization requirements (goal b)). Moreover, the horn power supply design allows to switch horn current polarity, and therefore neutrino sign selection (goal d)). 


\begin{tabular}{|c|c|}
\hline Material & aluminum alloy 6061-T6 \\
Inner conductor outer radius & $2.2-6.54 \mathrm{~cm}$ \\
Outer conductor inner radius & $30 \mathrm{~cm}$ \\
Length & $185 \mathrm{~cm}$ \\
\hline Peak current & $170 \mathrm{kA}$ \\
Maximum magnetic field & 1.5 Tesla \\
Current pulse duration (half-period) & $140 \mu \mathrm{s}$ \\
Voltage on horn & $3 \mathrm{kV}$ \\
Skin depth & $1.4 \mathrm{~mm}$ \\
\hline Average (maximum) repetition rate & $5(15) \mathrm{Hz}$ \\
Power dissipation by ohmic losses & $2.5 \mathrm{~kW}$ \\
Power dissipation by beam deposition & $0.8 \mathrm{~kW}$ \\
Cooling water flow rate & $1 \mathrm{l} / \mathrm{s}$ \\
\hline Design lifetime & $10^{8}$ pulses in $1 \mathrm{year}$ at $97.5 \% \mathrm{CL}$ \\
\hline
\end{tabular}

Table 3.1: Horn mechanical and electrical design properties.

Some of the most important horn mechanical and electrical design characteristics are listed in Tab. 3.1. The horn material is an aluminum alloy, particularly suitable for its high electrical conductivity, welding ability, resistance to corrosion, and low residual radio-activation. The geometrical shape of the horn, and in particular the shape of its inner conductor, is optimized by a full simulation for the expected neutrino fluxes at MiniBooNE. The inner conductor shape can be seen in the left panel of Fig. 3.3. The inner conductor is a $2.2 \mathrm{~cm}$ outer radius cylinder in the horn upstream half, while it is composed of two, larger radii (between 2.2 and $6.54 \mathrm{~cm}$ ), intersecting cones in the horn downstream half, to avoid over-focusing of positively-charged tracks produced at small angles within the target. The total horn length of $185 \mathrm{~cm}$, and the horn peak current of $170 \mathrm{kA}$, are also chosen based on simulations aimed at optimizing the MiniBooNE neutrino flux. The horn current pulse is a half-sinusoid with half- 

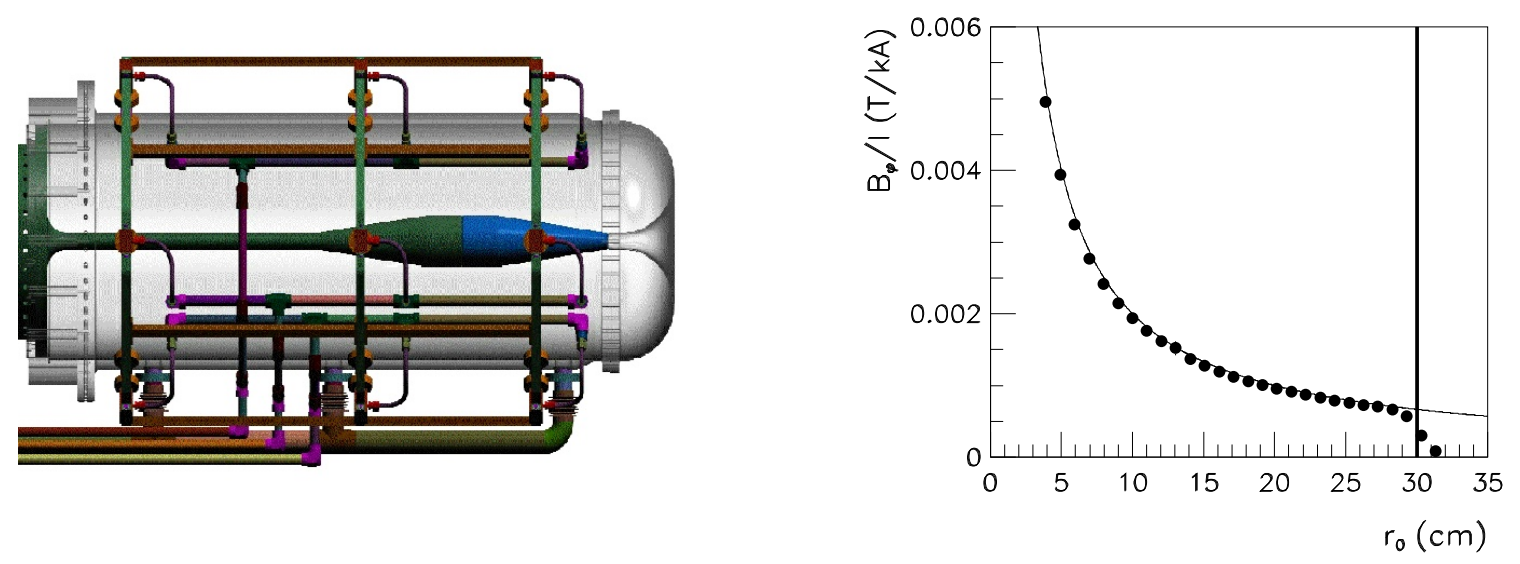

Figure 3.3: Left: drawing of the MiniBooNE horn and its water cooling system; the horn outer conductor surface is made transparent to show the horn inner conductor shape and the target. Right: points indicate measurements of the azimuthal component of the horn magnetic field $B_{\phi}$ divided by the peak horn current $I$, as a function of the distance $r$ from the horn axis. The solid line is the prediction from Eq. 3.2, and the solid vertical line is the inner radius of the horn outer conductor.

period $T / 2$ of approximately $140 \mu \mathrm{s}$, timed such that the $1.6 \mu \mathrm{s}$ long beam spill crosses the horn/target assembly during peak horn current. Given this horn current angular frequency $\omega=2 \pi / T$, and the aluminum permeability $\mu$ and conductivity $\sigma$, the skin depth $\delta=\sqrt{2 /(\mu \sigma \omega)}$ is $1.4 \mathrm{~mm}$, yielding a surface magnetic field inside the aluminum inner conductor with negligible impact on the neutrino fluxes.

The horn electrical circuit and mode of operation can be described, in its simplest terms, by an under-damped LCR circuit. The energy is initially stored in a capacitor bank with capacitance $\mathrm{C}=1,344 \mu \mathrm{F}$, charged by the horn power supply. Beam on target triggers the capacitor bank discharge, via silicon controlled rectifiers (SCR), into the horn load. The electrical connection between the power supply and the horn is provided by four, high-current transmission lines, characterized by a planar design (striplines) in order to minimize their inductance. The horn current pulse is given by:

$$
i(t)=\frac{V_{0}}{\omega L} e^{-\beta t} \sin \omega t
$$




\begin{tabular}{|c|r|r|r|}
\hline Component & Inductance $(\mu \mathrm{H})$ & Resistance $(\mathrm{m} \Omega)$ & Power $(\mathrm{kW})$ \\
\hline Capacitor bank plus losses & 0.37 & 3.00 & 31.0 \\
Transmission line & 0.34 & 0.27 & 2.8 \\
Horn & 0.70 & 0.24 & 2.5 \\
Total & 1.41 & 3.51 & 36.3 \\
\hline
\end{tabular}

Table 3.2: Resistive and inductive impedances of the various components of the horn electrical circuit, as well as power dissipation by ohmic losses for $5 \mathrm{~Hz}$ average repetition rate, $170 k A$ peak horn current operation.

where $V_{0} \simeq 6.0 \mathrm{kV}$ is the capacitor bank voltage corresponding to a peak horn current of $170 \mathrm{kA}, \beta=R /(2 L), \omega=\omega_{0} \sqrt{1-\gamma^{2}}, \omega_{0}=1 / \sqrt{L C}, \gamma=(R / 2) \sqrt{C / L}$, and $R, L$ are the resistive and inductive impedances of the horn circuit, summarized in Tab. 3.2. After the discharge, a separate circuit is used to recover most of the energy initially stored in the capacitor bank; the energy lost from ohmic losses is replaced by the charging power supply, in time for the next discharge cycle to begin. Under typical operations, a $15 \mathrm{~Hz}$ train of $5-10$ horn pulses is used, interleaved by a $\simeq 2 \mathrm{~s}$ hold-off time. The waveforms of the horn power supply voltage and current (including the charge recovery) can be seen in Fig. 3.4, showing one of the graphical interfaces used to monitor the horn operation.

In order to dissipate in an effective way the power deposited in the horn inner conductor by ohmic losses and beam energy loss, the MiniBooNE horn uses a water cooling system. Eighteen nozzles external to the horn outer conductor, and vibrationally isolated from the horn itself, continuously spray water on the horn inner conductor through openings in the horn outer conductor, at a flow rate of about 1 liter per second. The radioactive cooling water is kept in a closed circuit, equipped with a water to water heat exchanger to remove heat, and a filter to remove particulate content in the water.

Given this current pulse, the toroidal magnetic field between the horn inner and 


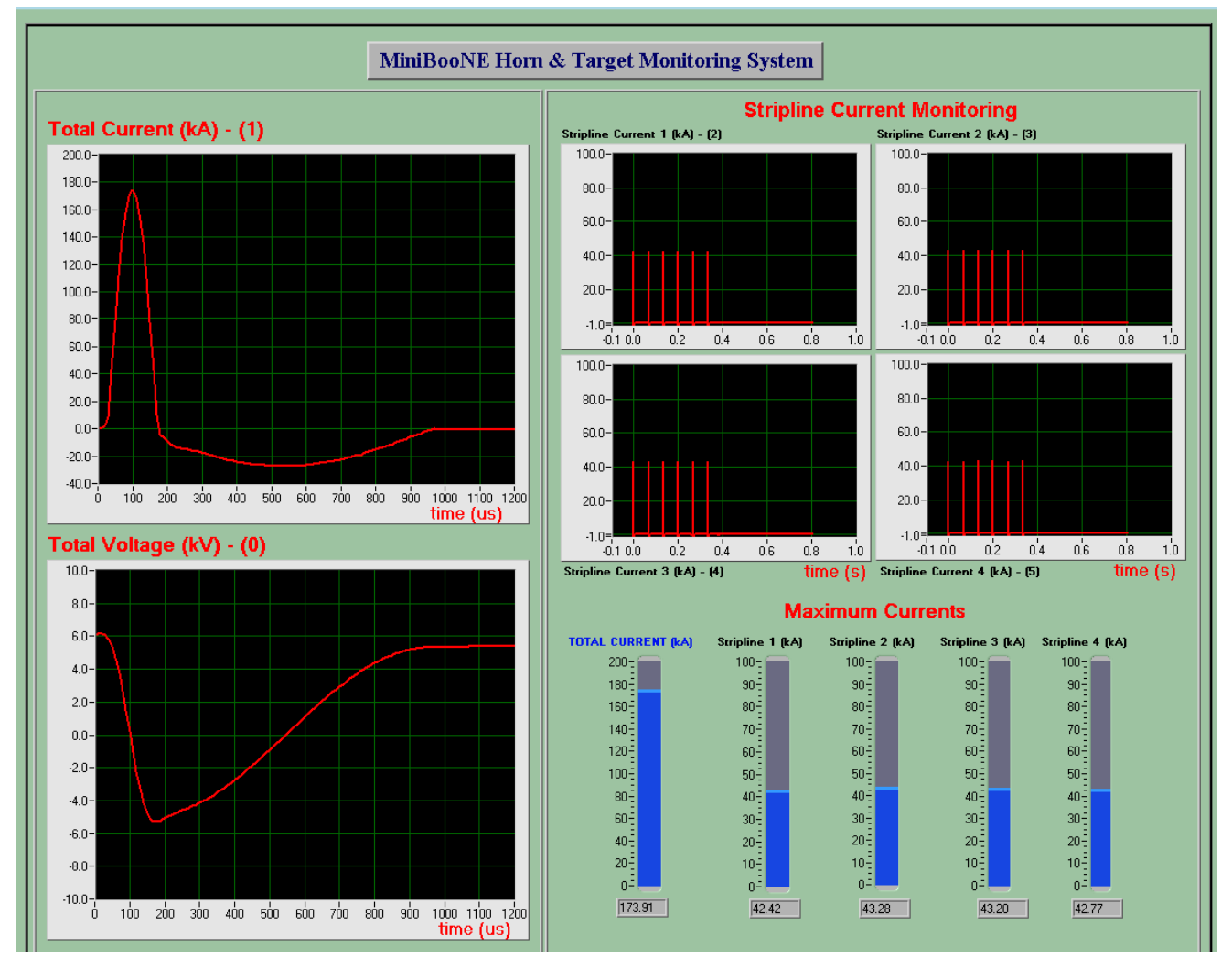

Figure 3.4: Current and voltage waveforms at the horn power supply.

outer conductors at peak horn current $I_{0} \simeq V_{0} \exp [-\pi \beta /(2 \omega)] /(\omega L)$ can be accurately approximated by the familiar expression for an infinitely long, straight conductor (in SI units):

$$
B_{\phi}=\frac{\mu_{0}}{2 \pi} \frac{I_{0}}{r}, B_{r}=B_{z}=0
$$

where $\mu_{0}$ is the magnetic permeability of air, and $r$ is the distance from the horn axis.

In order to quantify the performance of the MiniBooNE horn design summarized above, we define the neutrino focusing factor merit factor as:

$$
\stackrel{(-)}{F}(E)=\frac{\phi\left(E ; \stackrel{(-)}{\nu}_{\mu}\right)}{\phi_{0}\left(E ; \stackrel{(-)}{\nu}_{\mu}\right)}
$$

where $\phi_{0}\left(E ; \stackrel{(-)}{\nu}_{\mu}\right)$ is the simulated muon (anti)neutrino flux at the MiniBooNE detector for perfect focusing, and $\phi\left(E ; \stackrel{(-)}{\nu}_{\mu}\right)$ is the corresponding flux prediction for the MiniBooNE horn in various operating modes. Perfect focusing is defined here as the neutrino flux obtained by ideally transporting on axis all electrically-charged particles 

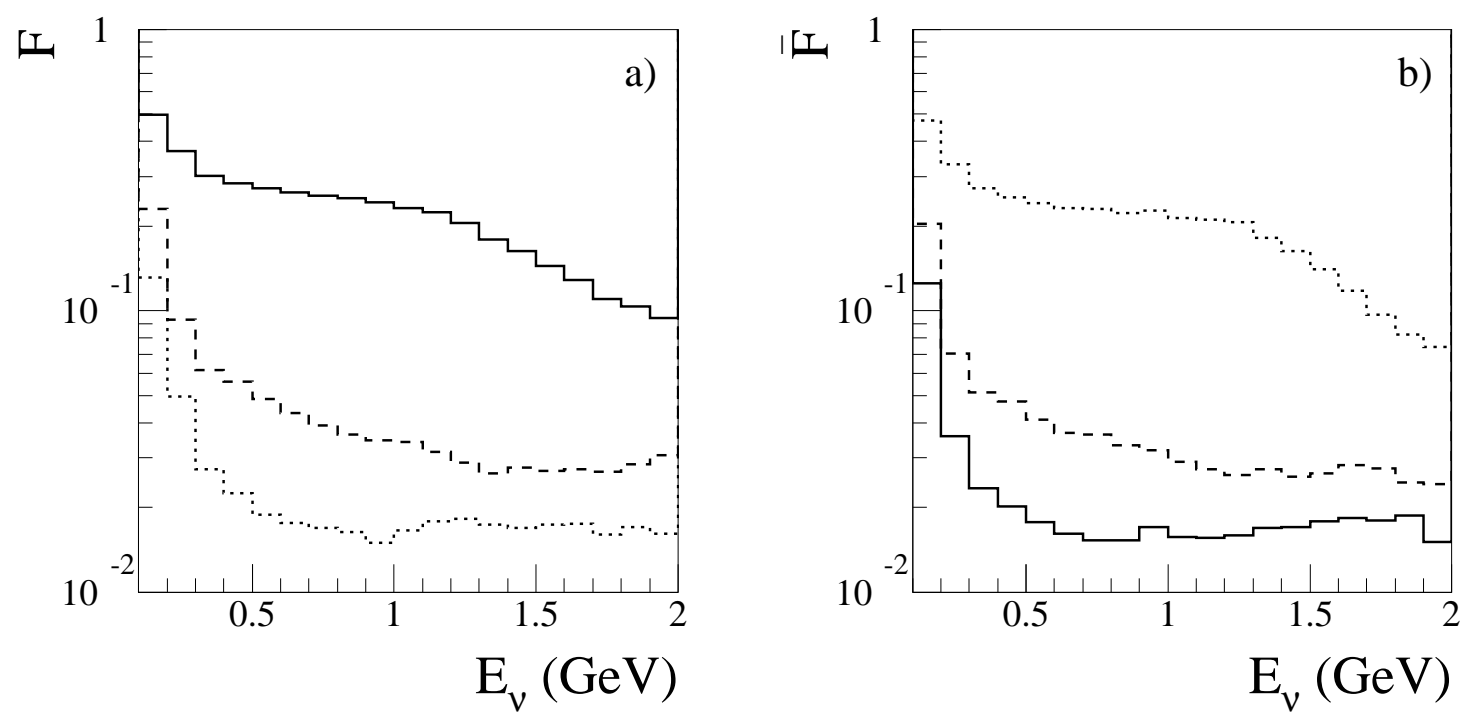

Figure 3.5: Focusing merit factor as a function of neutrino energy $E_{\nu}$ of the MiniBooNE horn for muon neutrinos (a)) and muon antineutrinos (b)), expressed as fraction of flux at the MiniBooNE detector for perfect neutrino focusing. The solid, dashed, dotted lines are for neutrino, horn-off, and antineutrino running modes, respectively.

reaching the downstream end of the horn, and simultaneously rotating their momentum vector to be at zero angle with respect to the beam axis. The horn operating conditions that are simulated here to quantify the horn performance are: neutrino running mode (+170 kA peak horn current), horn-off running mode (zero horn current), and antineutrino running mode (-170 kA peak horn current). The convention for the horn current flow taken here is such that a positive horn current corresponds to a current flowing along the horn inner conductor and then back along the outer conductor, thereby focusing positively-charged tracks.

The horn performance is evaluated with a full GEANT4 simulation of the MiniBooNE target/decay region that includes all physics processes relevant for neutrino production. This simulation is described in detail in Chapter 4. The MiniBooNE horn design is very effective in boosting the $\stackrel{(-)}{\nu}_{\mu}$ flux at the MiniBooNE detector, and in selecting either a neutrino or an antineutrino beam. Specifically, for typical muon neutrino energies of $0.7<E_{\nu}<0.8 \mathrm{GeV}$, the horn neutrino merit factor in 
neutrino running is predicted to be about one quarter, about six times higher than the neutrino merit factor in horn-off running (see Fig. 3.5 a); a similar merit factor is predicted for antineutrinos in antineutrino running (see Fig. 3.5b). Moreover, the merit factor for "wrong sign" neutrinos (e.g. antineutrinos in neutrino running) is about two times smaller than the corresponding factor in horn-off running, in the same neutrino energy range.

\subsubsection{Horn Testing}

Prior to installation in the beamline, each of the two horns built so far for the MiniBooNE experiment has been thoroughly tested. Tests include magnetic field, mechanical vibration, and cooling measurements, as well as a fatigue test.

A map of the horn magnetic field has been measured in more than 300 locations using an inductive coil [78. In a cylindrical coordinate system $(z, \varphi, r)$ with respect to the horn axis, the measurements show no significant dependence of the nonzero azimuthal component of the field as a function of the $z$ and $\varphi$ positions within the horn field region, while the expected $1 / r$ radial dependence of the field is confirmed in the explored radial range, $3.9 \mathrm{~cm}<r<30 \mathrm{~cm}$, as shown in the right panel of Fig. 3.3. Moreover, the measured longitudinal component of the field is consistent with zero, given the coil alignment accuracy achieved in the measurements.

Detailed simulations and measurements of the horn vibrational response to highcurrent pulses were also performed, and the impact of the horn mechanical stresses on the horn lifetime were evaluated. The studies allowed optimization of material thicknesses throughout the horn, in order to ensure adequate horn lifetime, while keeping to a minimal level the impact of particle interactions in aluminum on neutrino fluxes. The vibrational measurements indicated that, despite the high repetition rate, no pileup of horn vibrational modes from one current pulse to another occur.

The necessary water cooling flow rate was determined experimentally, by mea- 
suring the heat transfer coefficient from aluminum to water for spray cooling, in a test setup with a geometry identical to the MiniBooNE horn one. In the test setup, the inner conductor, withstanding power deposition by electric current and beam, was simulated with an electrically heated tube. The cooling measurements indicated that a water flow rate of the order of $1 \mathrm{l} / \mathrm{s}$ is sufficient to provide the necessary, $>3000 \mathrm{~W} /\left(\mathrm{m}^{2} \operatorname{deg} \mathrm{C}\right)$, heat transfer coefficient.

Finally, each MiniBooNE horn has successfully been subject to a fatigue test, consisting in pulsing the horn in nominal conditions ( $5 \mathrm{~Hz}$ average repetition rate, $170 \mathrm{kA}$ peak horn current), for a total of 10 million current pulses.

\subsubsection{Horn Performance}

Over the period between September, 2002, and January, 2005, more than $10^{8}$ proton batches were delivered from the Booster accelerator to the MiniBooNE target hall, with each proton batch triggering a $170 \mathrm{kA}$ peak current pulse on the MiniBooNE horn. The daily average repetition rate and the integrated number of horn pulses, as a function of time, are shown in Fig. 3.6.

As discussed above, the horn repetition rate over this running period has been determined mostly by the FNAL Booster accelerator mode of operation. In particular, the radiation concerns due to proton losses in the Booster did not permit pulsing of the horn at the design average repetition rate of $5 \mathrm{~Hz}$ throughout the run; the design repetition rate has been approached in recent operation. In the summer of 2004, approximately one month before a three-month long scheduled shutdown of the accelerator complex, the first MiniBooNE horn failed after $8.5 \cdot 10^{7}$ pulses due to a ground fault. The MiniBooNE horn/target assembly was successfully replaced during the summer 2004 shutdown, and the second horn has been pulsed more than $1.5 \cdot 10^{7}$ since then.

The stability of horn operation over time has also been investigated, and proved 


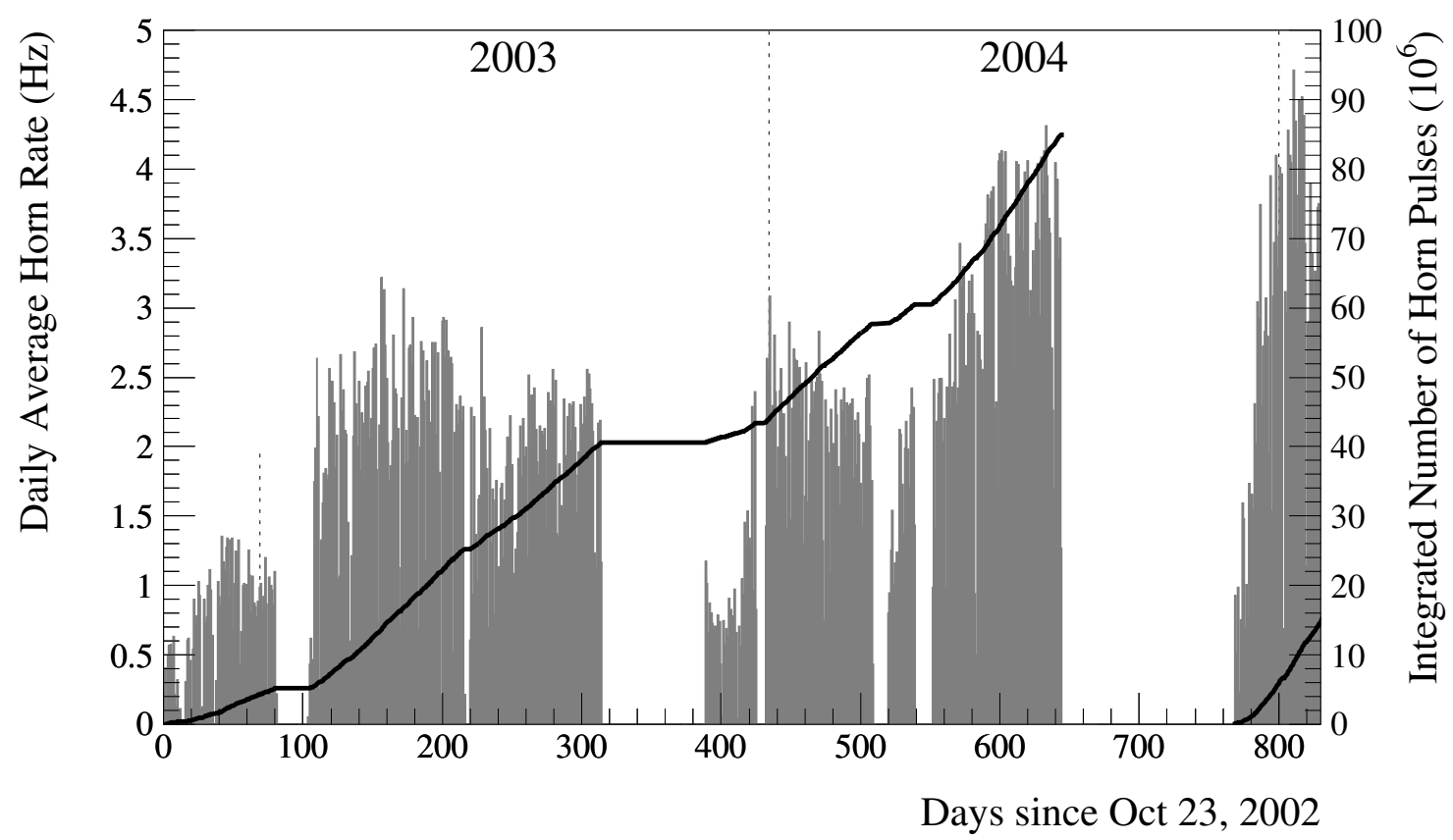

Figure 3.6: Performance of the MiniBooNE horn in the period between October, 2002, and January, 2005. The histogram shows the daily average horn repetition rate, the solid line the integrated number of horn pulses. In the Figure, the integrated number of pulses has been reset to zero after the horn replacement.

to be sufficiently reliable. The most important horn parameter to monitor in relation to physics analyses is the peak horn current. Fig. 3.7 shows the daily average peak horn current obtained by averaging over all horn pulses in the day. Over the more than two years of operation, the peak horn current has not changed by more than $1 \%$. It is estimated that a $1 \%$ variation in the peak horn current would cause only a $0.7 \%$ variation in the neutrino flux at the MiniBooNE detector.

In Section 3.2.1, we have described the expected focusing performance of the MiniBooNE horn as a function of neutrino energy, expressed in terms of fraction of perfect focusing neutrino flux. In this section, we discuss the measured rates of neutrino candidate events in the MiniBooNE detector per proton on target, for both horn-on, neutrino running mode, and horn-off mode.

MiniBooNE neutrino candidates are defined here in a very simple way, using exclusively MiniBooNE PMT hit multiplicity and timing, and no event reconstruction. 


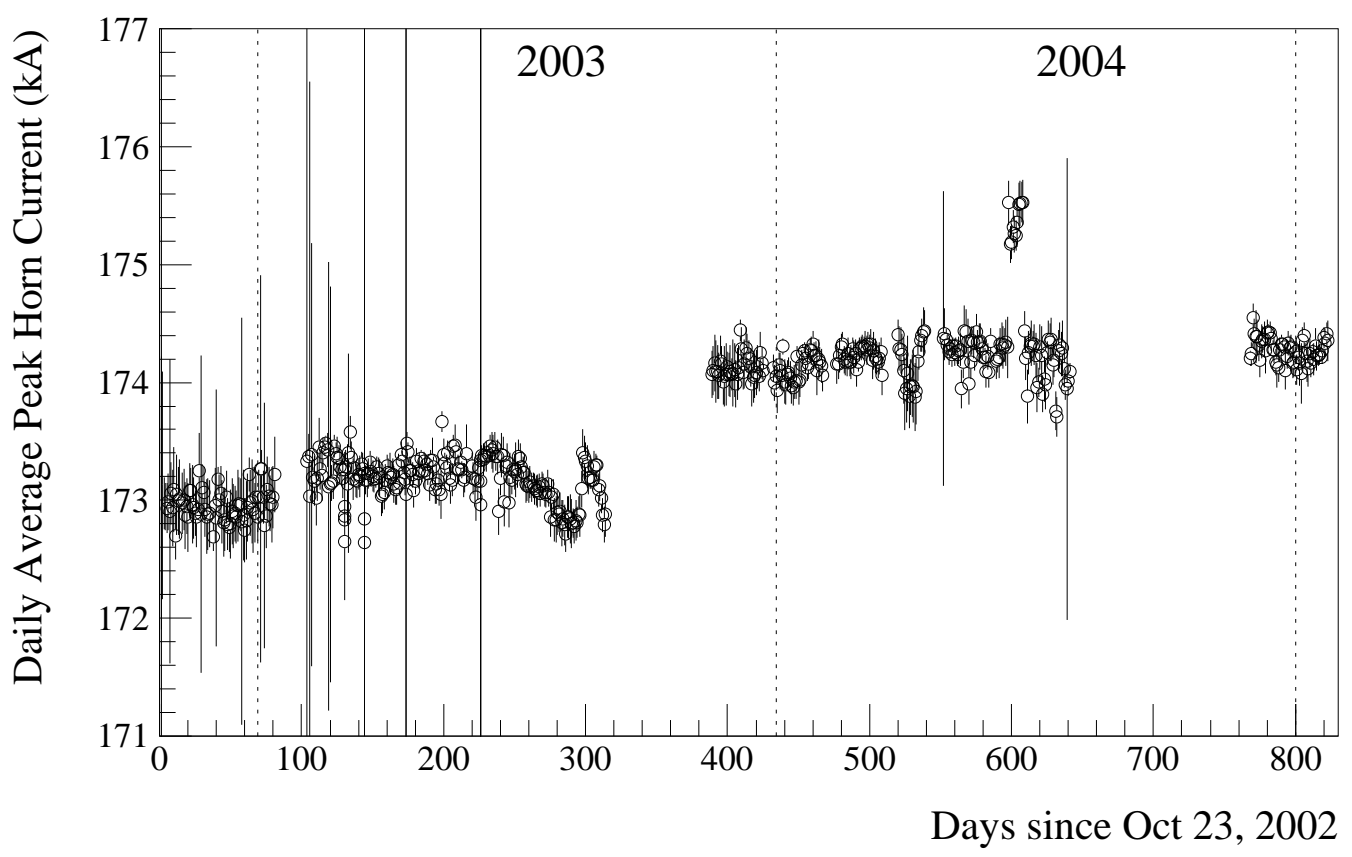

Figure 3.7: Performance of the MiniBooNE horn in the period between October, 2002, and January, 2005. The points and error bars show the mean and the RMS of the peak horn current averaged over one day.

Details on the MiniBooNE detector are given in Chapter 6, and only the detector aspects necessary to define neutrino candidates in this context are explained below. The sample of neutrino candidates is determined by triggering on beam on target, and uses the information on the PMT charges and times in the detector recorded over a $19.2 \mu$ s time interval. Next, the PMT activity is split into subevents, defined as clusters of time-related PMT hits of a typical duration of about 100 ns. The event splitting algorithm is introduced to tag the delayed light produced by electrons from muon decays, generally occurring several hundreds of nanoseconds after the neutrino interaction; the presence of electrons from muon decays in the detector generally cause the event to be split in $>1$ subevents. The simple neutrino event selection applied here requires less than 6 PMT hits in the veto region for the first subevent in the event, in order to reject cosmic ray muons crossing the detector in coincidence with beam activity. Moreover, the PMT hit multiplicity in the main detection region for the first subevent is required to be greater than 200 , in order to reject the $<52.3 \mathrm{MeV}$ electrons 


\begin{tabular}{|c|r|r|r|}
\hline Running Mode & $N_{\text {pot }}$ & $N_{\nu}$ & $N_{\nu} / N_{\text {pot }}$ \\
\hline Horn-on Neutrino & $1.95 \cdot 10^{20}$ & 218,337 & $1.120 \cdot 10^{-15}$ \\
Horn-off & $1.11 \cdot 10^{19}$ & 2,033 & $0.184 \cdot 10^{-15}$ \\
\hline
\end{tabular}

Table 3.3: Number of protons on target $N_{\text {pot }}$, number of neutrino candidate events $N_{\nu}$, and ratio of neutrino candidate events to protons on target $N_{\nu} / N_{\text {pot }}$, for selected periods of horn-on neutrino running mode and for horn-off running mode.

produced by the decays-at-rest of cosmic ray muons occurring before the $19.2 \mu$ s-long beam window. Overall, this simple event selection ensures a signal-to-background ratio in excess of 1000, where "signal" indicates here a neutrino-induced event, while "background" any other source of PMT activity in the MiniBooNE detector.

The number of protons on target are estimated from the readout of the toroid located immediately upstream of the MiniBooNE target, as discussed in Section 3.1.1. Moreover, beam quality cuts are applied to ensure that the protons hit the target, the horn fires, and that the toroid readout does not differ in a significant way from additional beam instrumentation located in the Booster neutrino beamline.

Table 3.3 shows the number of protons on target, the number of neutrino candidate events, and the neutrino-to-proton ratio measured in MiniBooNE in both horn-on and horn-off configurations over the running period October, 2002, to April, 2004. Most of the run time has been spent in horn-on, neutrino mode; two weeks were devoted to horn-off running mode in April, 2004. We observe a 6-fold increase in the neutrino candidate event rate per proton on target, $N_{\nu} / N_{\text {pot }}$ in Tab. 3.3 , by operating the horn in neutrino running mode, compared to horn-off mode.

This boost in neutrino interaction rate due to the horn focusing is crucial in order to accomplish the main physics goal of the MiniBooNE experiment discussed in Chapter 1, that is a $\nu_{\mu} \rightarrow \nu_{e}$ oscillation measurement with sufficient sensitivity in the neutrino mass and mixing parameter space indicated by the LSND signal. As mentioned in the previous Chapter, the MiniBooNE $\nu_{\mu} \rightarrow \nu_{e}$ sensitivity is dominated 
by the statistical uncertainty on the number of neutrino candidates, and therefore a 6 -fold increase in neutrino rate roughly corresponds to a $\sqrt{6}$ increase in sensitivity. 


\section{Chapter 4}

\section{MiniBooNE Neutrino Flux Predictions}

In this Chapter, predictions for the muon neutrino and electron neutrino fluxes at the MiniBooNE detector are presented. The program used to obtain the simulated neutrino fluxes, its physics assumptions, and the flux predictions are described in detail. Section 4.1 describes some generalities on the "beam Monte Carlo" code used; Section 4.2 discusses the simulation of the beamline geometry, materials, and of the primary proton beam; Sections 4.4, 4.5 and 4.6 summarize the physics input to the simulation for the processes of most direct relevance for producing muon and electron neutrinos at MiniBooNE; the flux predictions are given in Section 4.7.

\subsection{The Beam Monte Carlo Simulation Code}

\subsubsection{Inputs to Neutrino Flux Predictions}

The purpose of the MiniBooNE beam Monte Carlo is to predict the fluxes at the MiniBooNE detector for all relevant neutrino species $\left(\nu_{\mu}, \bar{\nu}_{\mu}, \nu_{e}, \bar{\nu}_{e}\right)$ as a function of neutrino energy, per proton on target and per unit area, and to obtain an accurate 
estimate of the associated flux systematic uncertainties. The flux understanding is of primary importance for most MiniBooNE analyses, including for example neutrino cross-section measurements, and $\nu_{\mu} \rightarrow \nu_{e}$ and $\nu_{\mu} \rightarrow \nu_{\mu}$ oscillation analyses.

The MiniBooNE flux prediction makes use of physics inputs both external and internal to the experiment. The most important physics input that is external to MiniBooNE is meson production data in proton-beryllium hadronic interactions for proton beam momenta in the $\sim 10 \mathrm{GeV} / \mathrm{c}$ range, since the flux uncertainty is dominated by the secondary meson production uncertainty. Therefore, the most important design requirement of the simulation tool developed at MiniBooNE for neutrino flux predictions is its flexible hadronic physics model interface.

In addition, the MiniBooNE data itself will provide inputs to the neutrino flux predictions. First, under the assumption of no neutrino oscillations, the observation of muon neutrinos at MiniBooNE will constrain the electron neutrino flux component due to muon decays [79]. Second, neutrino fluxes from kaon decays will be constrained by the measurement of muons from kaon decays in the Little Muon Counters along the decay region.

\subsubsection{Code Structure}

Currently, the combination of two simulation programs is used at MiniBooNE to obtain neutrino flux predictions. First, a GEANT4-based Monte Carlo code is responsible for simulating the chain of processes beginning with primary protons hitting the MiniBooNE target to the decay of secondary mesons and muons yielding neutrinos. GEANT4 [80, 81] is a software package designed to simulate the passage of particles through matter. In particular, the GEANT4 application used at MiniBooNE simulates:

1. the geometry and the materials present in the MiniBooNE target hall and decay region; 
2. the physics processes governing the interactions of baryons, mesons and leptons involved in neutrino production;

3. the generation of events, initiated by Booster primary protons specified according to the beam optics;

4. the tracking of particles through the materials present in the target hall and decay region, and through the horn magnetic field;

5. the storage of simulated data at various levels of refinement, from single physics process information, to event-level information for the entire neutrino parentage history.

A second, FORTRAN-based Monte Carlo code uses the output of the GEANT4 program as input, and is responsible for generating the neutrino kinematics distributions from meson and muon decays, and for obtaining the final neutrino fluxes extrapolated at the MiniBooNE detector with negligible beam Monte Carlo statistical error.

The physics interface of GEANT4 allows the user to choose among a large variety of built-in models, and also to easily implement custom-defined process cross-sections and final state descriptions, making GEANT4 an ideal simulation toolkit for the MiniBooNE beam Monte Carlo. The class category hierarchy of the $\mathrm{C}++$ based beam Monte Carlo code follows the schematics given in Fig. 4.1 (taken from [81]).

\subsection{Simulation of Beamline Geometry, Materials, and Proton Beam}

\subsubsection{Geometry and Materials}

The beam Monte Carlo program simulates the most relevant materials and volumes

present in the target hall and decay region. In Fig 4.2 , four images from the beamline 


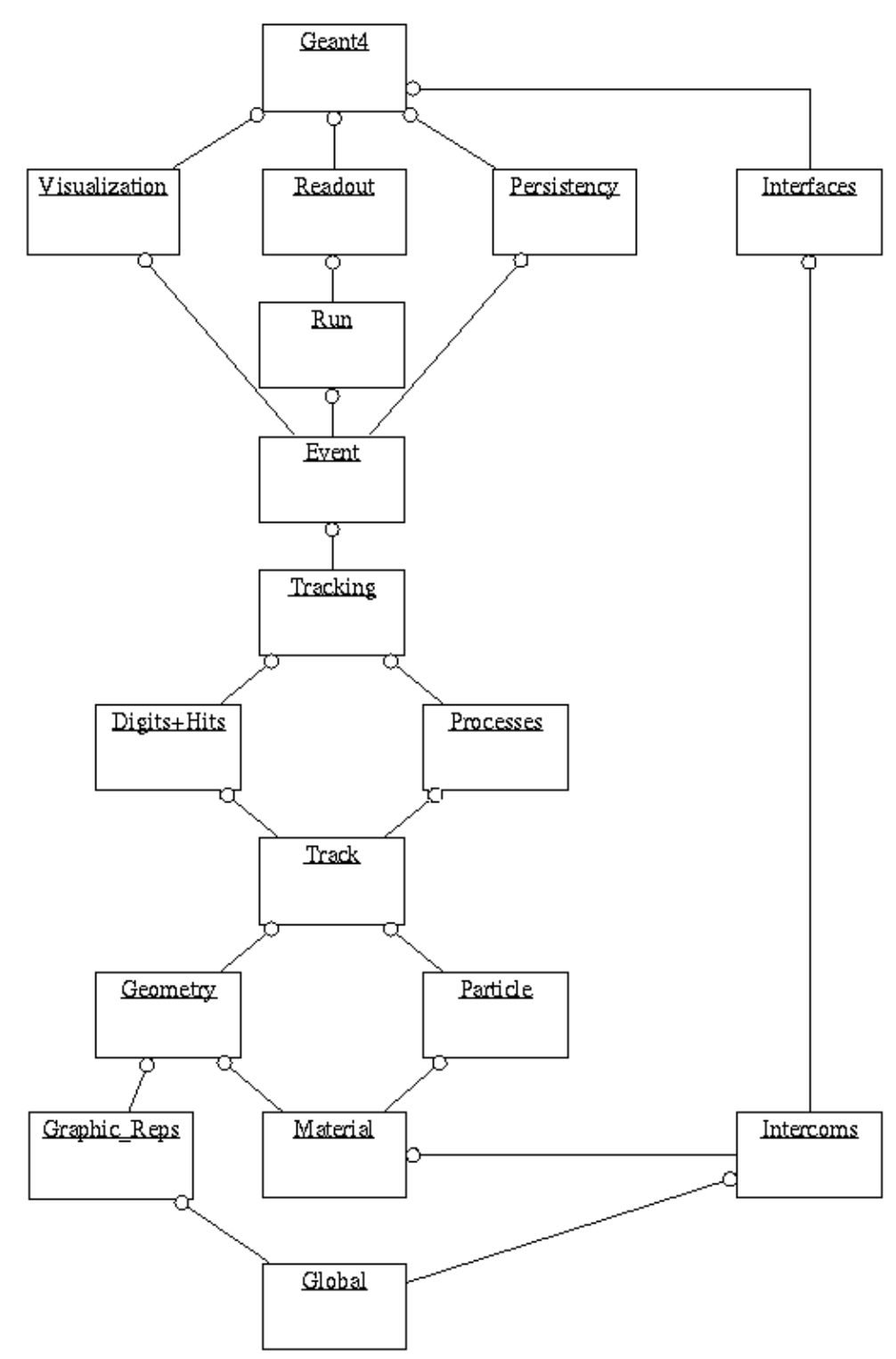

Figure 4.1: GEANT4 class categories [81]. Each box represents a class category. The circle at the end of a straight line means that the class category which has this circle uses the other line-connected category.

geometry simulation are shown. The images show the entire decay region, and details of the target hall region, horn, and target. The volumes shown in red, blue, green are made of beryllium, iron, and aluminum, respectively: the target is made of beryllium, the horn is made of aluminum, the target hall shielding and collimation system, and the absorber located at the end of the decay region, are made of iron. Concrete is 


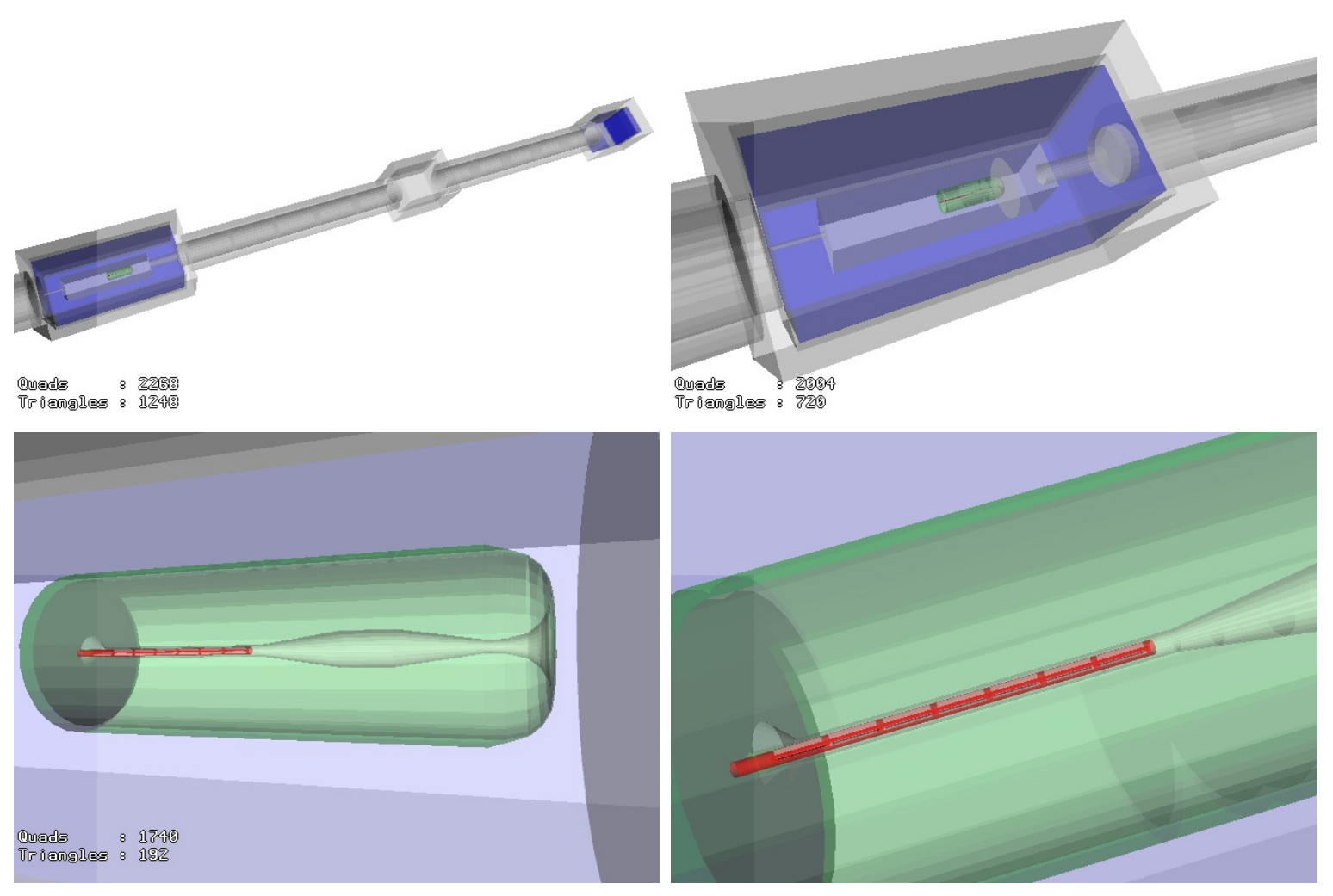

Figure 4.2: The MiniBooNE beamline geometry implemented in the simulation. The pictures show the entire $50 \mathrm{~m}$ decay region (top left), and details of the target hall region (top right), horn (bottom left), and target (bottom right).

used for the decay region walls, and concrete is also present in the target hall and absorber regions.

\subsubsection{Primary Proton Event Generator}

The event generator in the beam Monte Carlo simulation is very simple: one primary proton per event is generated with $8 \mathrm{GeV}$ kinetic energy, directed toward the beryllium target. The beam optics are realistically simulated by introducing some smearing in the initial transverse position $(x, y)$ and angular divergence $\left(\theta_{x}, \theta_{y}\right)$ of the beam, in the coordinate system where the $z$ axis points along the beam direction, and $y$ points upwards. Also, the mean $\left(x_{0}, y_{0}\right)$ positions and the mean $\left(\theta_{x, 0}, \theta_{y, 0}\right)$ directions 
of the primary beam can be changed for systematic study purposes, together with the longitudinal position $z_{0}$ for the primary proton at birth; all beam optics parameters refer to this longitudinal position. The position and angular smearing are described by Gaussian distributions. More specifically, the initial position and momentum of the generated primary proton are 1 .

$$
\left\{\begin{array}{l}
x=x_{0}+\sigma_{x} \mathrm{ran} 1 \\
y=y_{0}+\sigma_{y} \mathrm{ran} 2 \\
z=z_{0} \\
p_{x}=\sqrt{E_{p}^{2}-m_{p}^{2}}\left(\theta_{x, 0}+\sigma_{\theta_{x}} \mathrm{ran} 3\right) \\
p_{y}=\sqrt{E_{p}^{2}-m_{p}^{2}}\left(\theta_{y, 0}+\sigma_{\theta_{y}} \mathrm{ran} 4\right) \\
p_{z}=\sqrt{E_{p}^{2}-m_{p}^{2}-p_{x}^{2}-p_{y}^{2}}
\end{array}\right.
$$

where ran1, ran2, ran3, ran4 are four random numbers drawn from a gaussian distribution of mean zero and variance one, $E_{p}=K_{p}+m_{p}$, where $K_{p}=8 \mathrm{GeV}$ and $m_{p}$ is the proton mass. The MiniBooNE beam optics parameters $\left(x_{0}, \sigma_{x}, y_{0}, \sigma_{y}, z_{0}, \theta_{x, 0}, \sigma_{\theta_{x}}\right.$, $\left.\theta_{y, 0}, \sigma_{\theta_{y}}\right)$ used in the flux simulation are given in Tab. 4.1. These values are determined from detailed simulations of Booster protons in the $8 \mathrm{GeV}$ Fermilab neutrino line, and cross-checked with measurements from several beam detectors measuring beam positions, directions, and profiles at various locations along the beamline, as discussed in Chapter 3. It is estimated that this choice of beam optics parameters yields a $99.8 \%$ targeting efficiency, in good agreement with the design parameters.

\footnotetext{
${ }^{1}$ The small-angle approximation is assumed throughout the simulation of primary beam parameters.
} 


\begin{tabular}{|c|c|c|c|c|c|c|c|c|}
\hline Parameter & $x_{0}$ & $\sigma_{x}$ & $y_{0}$ & $\sigma_{y}$ & $\theta_{x, 0}$ & $\sigma_{\theta, x}$ & $\theta_{y, 0}$ & $\sigma_{\theta, y}$ \\
\hline Value & 0 & $1.51 \mathrm{~mm}$ & 0 & $0.75 \mathrm{~mm}$ & 0 & $0.66 \mathrm{mrad}$ & 0 & $0.40 \mathrm{mrad}$ \\
\hline
\end{tabular}

Table 4.1: Beam optics parameters used in the beam Monte Carlo simulation. See text for definitions. The parameters correspond to an initial longitudinal position along the beam direction for the generated protons of $z_{0}=-1 \mathrm{~cm}$, and to a coordinate system having the MiniBooNE beryllium target located at: $3.5 \mathrm{~cm}<z<74.6 \mathrm{~cm}, \sqrt{x^{2}+y^{2}}<4.76 \mathrm{~cm}$.

\begin{tabular}{|c|c|c|c|c|}
\hline Neutrino Flavor & $\nu_{\mu}$ & $\bar{\nu}_{\mu}$ & $\nu_{e}$ & $\bar{\nu}_{e}$ \\
\hline Flux fraction (\%) & 92.7 & 6.6 & 0.6 & 0.1 \\
\hline
\end{tabular}

Table 4.2: Neutrino flavor composition of the MiniBooNE neutrino flux in neutrino running, as predicted by the beam Monte Carlo simulation.

\subsection{Properties of Typical Neutrinos Reaching the MiniBooNE Detector}

In Sections 4.4, 4.5, 4.6, we discuss several physics processes of relevance for MiniBooNE flux predictions. As an introduction to these sections, we first discuss some properties of typical events yielding neutrinos at the MiniBooNE detector, as predicted by the beam Monte Carlo simulation. This Section gives some qualitative idea of what "relevant" means.

Table 4.2 shows the predicted neutrino flavor composition of the MiniBooNE neutrino flux in neutrino running mode. The flux is dominated by muon neutrinos, comprising $92.7 \%$ of the total neutrino flux, with a $6.6 \%$ flux contribution from muon antineutrinos. Electron neutrinos comprise about $0.6 \%$ of the total neutrino flux.

Table 4.3 shows the most likely "neutrino history" for neutrinos reaching MiniBooNE, per neutrino flavor. The muon neutrino flux is mostly $(86.1 \%)$ due to the decay of positive pions, which are produced directly by inelastic interactions of primary protons. The contribution of muon neutrinos from pions created by secondary 


\begin{tabular}{|c|c|c|}
\hline Neutrino flavor & Process & $\begin{array}{c}\text { Flux fraction } \\
\text { per flavor }(\%)\end{array}$ \\
\hline$\nu_{\mu}$ & $\begin{array}{c}p \rightarrow \pi^{+} \rightarrow \nu_{\mu} \\
p \rightarrow p \rightarrow \pi^{+} \rightarrow \nu_{\mu} \\
p \rightarrow K^{+} \rightarrow \nu_{\mu} \\
p \rightarrow n \rightarrow \pi^{+} \rightarrow \nu_{\mu} \\
\text { Other }\end{array}$ & $\begin{array}{r}86.1 \\
7.3 \\
2.8 \\
1.9 \\
1.9\end{array}$ \\
\hline $\bar{\nu}_{\mu}$ & $\begin{array}{c}p \rightarrow \pi^{-} \rightarrow \bar{\nu}_{\mu} \\
p \rightarrow p \rightarrow \pi^{-} \rightarrow \bar{\nu}_{\mu} \\
p \rightarrow n \rightarrow \pi^{-} \rightarrow \bar{\nu}_{\mu} \\
\text { Other }\end{array}$ & $\begin{array}{l}55.0 \\
16.6 \\
12.0 \\
16.4\end{array}$ \\
\hline$\nu_{e}$ & $\begin{array}{c}p \rightarrow \pi^{+} \rightarrow \mu^{+} \rightarrow \nu_{e} \\
p \rightarrow K^{+} \rightarrow \nu_{e} \\
p \rightarrow K_{L}^{0} \rightarrow \nu_{e} \\
p \rightarrow p \rightarrow \pi^{+} \rightarrow \mu^{+} \rightarrow \nu_{e} \\
\text { Other }\end{array}$ & $\begin{array}{r}47.6 \\
32.7 \\
7.2 \\
5.0 \\
7.5\end{array}$ \\
\hline $\bar{\nu}_{e}$ & $\begin{array}{c}\quad p \rightarrow K_{L}^{0} \rightarrow \bar{\nu}_{e} \\
p \rightarrow \pi^{-} \rightarrow \mu^{-} \rightarrow \bar{\nu}_{e} \\
\text { Other }\end{array}$ & $\begin{array}{r}65.5 \\
9.8 \\
24.7\end{array}$ \\
\hline
\end{tabular}

Table 4.3: Most likely history for all neutrino types reaching the MiniBooNE detector, as simulated by the beam Monte Carlo. The arrows indicate either an inelastic interaction, or a decay.

protons and neutrons is also non-negligible, as is non-negligible the contribution of muon neutrinos from charged kaon decays. Electron neutrino production is dominated by the decays of muons produced by positive pions, which are in turn directly produced in inelastic interactions of primary protons $(48.0 \%$ of the total electron neutrino flux). Contributions to the electron neutrino flux from charged and neutral kaon decays, and from other muon decay chains, are also significant. 


\begin{tabular}{|c|c|c|}
\hline Quantity & Description & Value \\
\hline$\left\langle p_{\pi+, i n}\right\rangle$ & Initial $\pi^{+}$momentum & $2.17 \mathrm{GeV} / \mathrm{c}$ \\
$\left\langle\vartheta_{\pi+, i n}\right\rangle$ & Initial $\pi^{+}$angle & $106 \mathrm{mrad}$ \\
$\left\langle l_{\pi+, B}\right\rangle$ & $\pi^{+}$path in horn field & $82.1 \mathrm{~cm}$ \\
$\left\langle B_{\pi+}\right\rangle$ & Horn field along $\pi^{+}$trajectory & $0.71 \mathrm{Tesla}$ \\
$\left\langle p_{\pi+, \text { fin }}\right\rangle$ & Final $\pi^{+}$momentum & $2.08 \mathrm{GeV} / \mathrm{c}$ \\
$\left\langle\vartheta_{\pi+, \text { fin }}\right\rangle$ & Final $\pi^{+}$angle & $30 \mathrm{mrad}$ \\
\hline$\left\langle E_{\nu}\right\rangle$ & Neutrino energy & $0.762 \mathrm{GeV}$ \\
\hline
\end{tabular}

Table 4.4: Average properties for $p \rightarrow \pi^{+} \rightarrow \nu_{\mu}$ processes yielding muon neutrinos at MiniBooNE, as simulated by the beam Monte Carlo. The properties listed illustrate pion production, focusing, and decay kinematics characteristics.

The remaining two tables in this section focus on muon neutrinos, and in particular on the $p \rightarrow \pi^{+} \rightarrow \nu_{\mu}$ events that mainly contribute to the total muon neutrino flux. Table 4.4 lists average properties concerning pion production, focusing, and pion decay kinematics. Pions yielding muon neutrinos at MiniBooNE are produced in the beryllium target with an average momentum and angle of $\sim 2.2 \mathrm{GeV} / \mathrm{c}$ and $\sim 100$ mrad, respectively. Pions lose a small fraction of energy crossing various materials, and are focused by a $\sim 1$ Tesla horn magnetic field over distances of the order of $\sim 1$ meter, as well as by the collimator system, resulting in average pion angles at decay of about 30 mrad. Neutrinos reaching the MiniBooNE detector are produced forward in the pion center-of-mass frame, resulting in typical neutrino energies that are a little less than the maximum neutrino energy of $E_{\nu}=\left(m_{\pi}^{2}-m_{\mu}^{2}\right) E_{\pi} / m_{\pi}^{2}$, where $m_{\pi}$ and $m_{\mu}$ are the $\pi^{+}$and $\mu^{+}$masses, respectively, and $E_{\pi}$ is the total $\pi^{+}$energy at decay.

Table 4.5 shows other properties for $p \rightarrow \pi^{+} \rightarrow \nu_{\mu}$ events yielding muon neutrinos at MiniBooNE, related to beamline geometry, energy loss, and pion decay characteristics. Primary protons travel about $22 \mathrm{~cm}$ on average in the Be target be- 


\begin{tabular}{|c|c|r|c|}
\hline Particle & Material & $\langle\Delta E\rangle(\mathrm{MeV})$ & $\langle l\rangle(\mathrm{cm})$ \\
\hline$p$ & beryllium & 56.3 & 22.3 \\
\hline$\pi^{+}$ & aluminum & 22.3 & 5.9 \\
$\pi^{+}$ & beryllium & 21.5 & 8.5 \\
$\pi^{+}$ & iron & 11.3 & 1.1 \\
$\pi^{+}$ & concrete & 6.5 & 2.9 \\
$\pi^{+}$ & air & 2.9 & 1900 \\
\hline
\end{tabular}

Table 4.5: Average properties for $p \rightarrow \pi^{+} \rightarrow \nu_{\mu}$ processes yielding muon neutrinos at MiniBooNE, as simulated by the beam Monte Carlo. The properties listed illustrate beamline geometry, energy loss, and pion decay characteristics, and are classified per beamline material. In the Table, $\langle E\rangle$ is the average particle energy loss, and $\langle l\rangle$ is the average particle pathlength in the material.

fore producing positive pions. The pions that are produced by protons can interact via several physics processes (see Sections 4.4, 4.5) while crossing a few centimeters of beryllium and aluminum, before decaying into neutrinos after typical pathlengths of $20 \mathrm{~m}$ in air.

In the following Sections 4.4, 4.5, 4.6, we quantify the agreement between the physics inputs to the beam Monte Carlo simulation, with expectations drawn from experimental data points, data-driven parametrizations, and theoretical predictions. Each physics input is tested individually, by disabling all other physics processes in the simulation. 


\subsection{Hadronic Interactions}

\subsubsection{Inelastic Interactions of Primary Protons in the Mini- BooNE Target}

An accurate simulation of inelastic interactions of primary protons in the MiniBooNE target material is essential for obtaining accurate neutrino flux predictions. For this reason, a custom-defined description of both the total proton-beryllium inelastic crosssection, and of the final state for these inelastic interactions, is used at MiniBooNE.

The proton-beryllium total inelastic cross-section assumed in the simulation for $8.9 \mathrm{GeV} / \mathrm{c}$ Booster protons is $\sigma_{\text {inel }}=189.3 \mathrm{mb}$, as obtained from an interpolation of the cross-sections measured in the BNL E910 experiment at 6.4 and $12.3 \mathrm{GeV} / \mathrm{c}$ proton beam momenta [82]. This value corresponds to an inelastic interaction length for protons in beryllium of $\lambda_{\text {inel }}=42.3 \mathrm{~cm}$. The impact of varying this cross-section value within its measured uncertainty is taken into account in the estimate of the flux systematic uncertainty.

Concerning the final state in inelastic proton-beryllium interactions, the seven types of secondaries of most direct relevance for neutrino fluxes are simulated: $\pi^{+}, \pi^{-}$, $K^{+}, K^{-}, K_{L}^{0}$, protons and neutrons. For each inelastic interaction, the multiplicities and kinematic distributions for the first three particle types are drawn from three parametrizations of double-differential, inclusive production cross-sections described next, while production cross-sections obtained from MARS15 [83, 84] simulations are assumed for the latter four particle types ${ }^{2}$.

\footnotetext{
${ }^{2}$ Strictly speaking, neutral kaon production information is not available from MARS15, at this time. Therefore, neutral kaon production is simulated according to the MARS15 positive kaon production, times the neutral-to-positive kaon production from the GFLUKA model, via $d^{2} N\left(K_{L}^{0}\right) / d p_{z} d p_{t}=\left(d^{2} N\left(K^{+}\right) / d p_{z} d p_{t}\right)_{\mathrm{MARS}} \cdot\left[\left(d^{2} N\left(K_{L}^{0}\right) / d p_{z} d p_{t}\right) /\left(d^{2} N\left(K^{+}\right) / d p_{z} d p_{t}\right)\right]_{\mathrm{GFLUKA}}$, where $p_{z}, p_{t}$ are the kaon logitudinal and transverse momentum components, and GFLUKA refers to the FLUKA hadron production model [85] used by the GEANT3.21 simulation program [86].
} 


\begin{tabular}{|c|c|c|c|c|c|c|c|c|c|}
\hline Secondary & \multicolumn{10}{|c|}{ Sanford-Wang parameter } \\
Particle & $c_{1}$ & $c_{2}$ & $c_{3}$ & $c_{4}$ & $c_{5}$ & $c_{6}$ & $c_{7}$ & $c_{8}$ & $c_{9}$ \\
\hline$\pi^{+}$ & 206.4 & 1.030 & 5.902 & 2.012 & 2.127 & 5.510 & $0.9958 \mathrm{E}-01$ & 12.03 & 1.000 \\
$\pi^{-}$ & 184.1 & 1.052 & 6.706 & 1.275 & 1.424 & 5.225 & $0.9439 \mathrm{E}-01$ & 10.74 & 1.000 \\
$K^{+}$ & 12.53 & 1.654 & 0.314 & 1.038 & 0.174 & 4.658 & 0.106 & 10.53 & 2.635 \\
\hline
\end{tabular}

Table 4.6: Values for the Sanford-Wang $\pi^{ \pm}$and $K^{+}$production parameters used in the beam Monte Carlo simulation to describe meson production in proton-beryllium interactions [82].

The double-differential, inclusive production cross-sections for the secondaries $S=\pi^{+}, \pi^{-}, K^{+}$are described according to "Sanford-Wang" parametrizations [87]:

$$
\begin{gathered}
\frac{d^{2} \sigma(p+B e \rightarrow S+X)}{d p d \Omega}= \\
=c_{1} p^{c_{2}}\left(1-\frac{p}{p_{\text {beam }}-c_{9}}\right) \exp \left[-c_{3} \frac{p^{c_{4}}}{p_{\text {beam }}^{c_{5}}}-c_{6} \vartheta\left(p-c_{7} p_{\text {beam }} \cos ^{c_{8}} \vartheta\right)\right]
\end{gathered}
$$

where $X$ means any other particle in the final state, $p_{\text {beam }}$ is the proton beam momentum in $\mathrm{GeV} / \mathrm{c}, p$ and $\theta$ are the secondary momentum and angle in units of $\mathrm{GeV} / \mathrm{c}$ and radians, respectively, $d^{2} \sigma /(d p d \Omega)$ is expressed in units of $\mathrm{mb} /(\mathrm{GeV} / \mathrm{c} \mathrm{sr})$, and the parameters $c_{1}, \ldots, c_{9}$ are empirical parameters obtained from fits to meson production data. The parameter $c_{9}$, related to the momentum threshold for meson production, is not fit in the case of pions, but set to $1 \mathrm{GeV} / \mathrm{c}$. The value of these parameters assumed by the beam Monte Carlo simulation are given in Tab. 4.6. Concerning muon (anti)neutrino fluxes, the simulation of $\pi^{+}$production is particularly important in the current MiniBooNE neutrino running mode, while a correct $\pi^{-}$production simulation would be essential for a possible future MiniBooNE antineutrino run. On the other hand, the knowledge of $K^{+}$production affects the estimate for the amount of intrinsic electron neutrinos in the beam from $K^{+}$decays.

The Sanford-Wang parameters given in Tab. 4.6 are obtained from fits of meson production data from a number of hadron production experiments, with proton beam momenta similar to the Booster proton momentum, and sensitive to a phase space for meson production of relevance to MiniBooNE. A summary of the data used in the 


\begin{tabular}{|c|r|r|r|r|r|r|r|}
\hline Secondary & Proton beam & \multicolumn{3}{|c|}{ Secondary particle phase space } & Data & Ref. \\
particle $S$ & $\begin{array}{r}\text { momentum } \\
(\mathrm{GeV} / \mathrm{c})\end{array}$ & $\begin{array}{r}\theta_{S, \text { min }} \\
(\mathrm{mrad})\end{array}$ & $\theta_{S, \text { max }}$ & $\begin{array}{r}p_{S, \text { min }} \\
(\mathrm{mrad})\end{array}$ & $\begin{array}{r}p_{S, \text { max }} \\
(\mathrm{GeV} / \mathrm{c})\end{array}$ & $\begin{array}{c}\text { points } \\
(\mathrm{GeV} / \mathrm{c})\end{array}$ & \\
\hline \multirow{2}{*}{$\pi^{+}$} & 12.3 & 42 & 331 & 0.6 & 5.4 & 71 & {$[82]$} \\
& 12.4 & 2 & 206 & 2.1 & 6.3 & 53 & {$[87]$} \\
& 6.4 & 71 & 353 & 0.6 & 4.2 & 29 & {$[82]$} \\
& 10.1 & 61 & 61 & 1.0 & 4.5 & 14 & {$[88$} \\
\hline \multirow{2}{*}{$\pi^{-}$} & 12.3 & 42 & 331 & 0.6 & 5.4 & 70 & {$[82]$} \\
& 12.4 & 2 & 206 & 2.1 & 6.3 & 32 & {$[87]$} \\
& 6.4 & 71 & 353 & 0.6 & 4.2 & 28 & {$[82]$} \\
\hline \multirow{2}{*}{$K^{+}$} & 19.2 & 13 & 70 & 6.0 & 16.0 & 41 & {$[89]$} \\
& 12.3 & 0 & 175 & 0.5 & 1.0 & 9 & {$[90]$} \\
& 9.5 & 62 & 62 & 3.0 & 6.5 & 5 & {$[91]$} \\
\hline
\end{tabular}

Table 4.7: Summary of hadro-production data used to obtain the MiniBooNE, SanfordWang parametrizations of the double-differential, meson production inclusive cross-sections.

MiniBooNE Sanford-Wang fits is given in Tab. 4.7.

While some data used in the MiniBooNE Sanford-Wang fits is available in the literature, the dominant contribution (in terms of statistical power) for $\pi^{ \pm}$production is based on a new analysis of Brookhaven E910 data at 6.4 and $12.3 \mathrm{GeV} / \mathrm{c}$ proton beam momenta on a thin beryllium target, done by collaborators from both experiments. Figure 4.3 shows a comparison between E910 $12.3 \mathrm{GeV} / \mathrm{c}$ data on $\pi^{+}$production, and the parametrization assumed in the beam Monte Carlo simulation. In the near future, meson production data from the CERN HARP experiment [92, obtained with beryllium targets of various thicknesses and at the Booster beam momentum, will also be used as inputs to the beam Monte Carlo simulation. The uncertainties in meson production data obtained from the Sanford-Wang fits are propagated into neutrino flux systematic uncertainties.

Given the assumptions described above, the particle multiplicities per inelastic interaction for all simulated secondaries are given in Tab. 4.8. The average multi- 

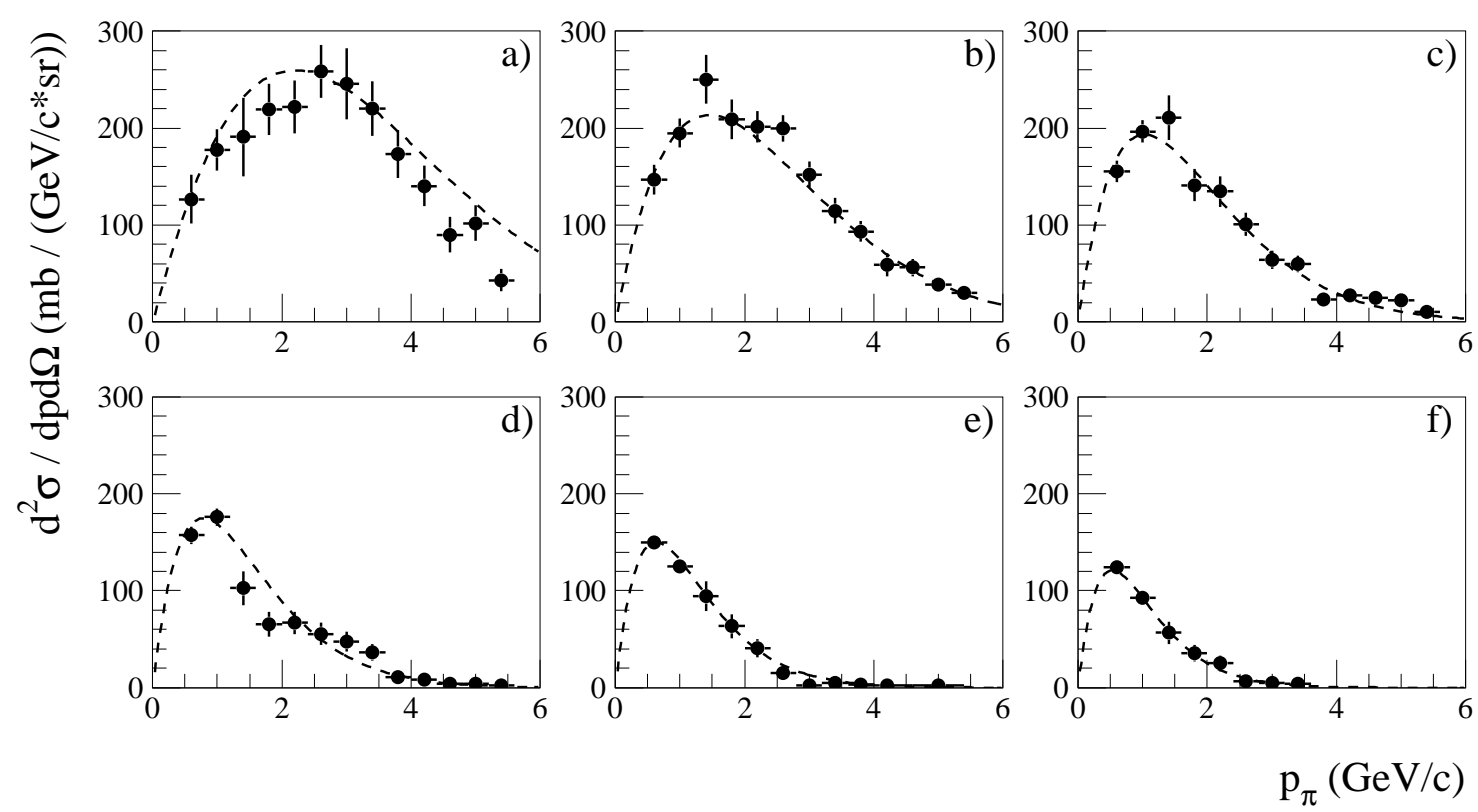

Figure 4.3: The double-differential $\pi^{+}$production cross-section in proton-beryllium interactions, as a function of pion production momentum $p_{\pi}$ and angle $\theta_{\pi}$. The six panels correspond to: $\theta_{\pi}(\mathrm{mrad})=42(\mathrm{a}), 95(\mathrm{~b}), 153(\mathrm{c}), 212(\mathrm{~d}), 272(\mathrm{e}), 331(\mathrm{f})$. The points correspond to BNL E910 data at peam $=12.3 \mathrm{GeV} / \mathrm{c}$ [82]; the dashed lines correspond to the beam Monte Carlo $\pi^{+}$production assumption, specified by Eq. 4.2 and Tab. 4.6, for the same $12.3 \mathrm{GeV} / \mathrm{c}$ beam momentum.

\begin{tabular}{|c|c|r|r|r|r|r|r|}
\hline Secondary particle type & $\pi^{+}$ & $\pi^{-}$ & $K^{+}$ & $K^{-}$ & $K_{L}^{0}$ & $p$ & $n$ \\
\hline Average multiplicity & 0.799 & 0.596 & 0.048 & 0.003 & 0.030 & 1.544 & 1.341 \\
\hline
\end{tabular}

Table 4.8: Average multiplicity per inelastic collision for secondary particles produced in inelastic collisions of $8.9 \mathrm{GeV} / \mathrm{c}$ protons in the MiniBooNE beryllium target.

plicities per inelastic collision are defined as the integral of the double-differential, inclusive production cross-section over the entire secondaries phase space, divided by the total proton-beryllium inelastic cross-section. Proton and neutron production are the most abundant, followed by $\pi^{+}$production.

Figure 4.4 shows the one-dimensional projections along the longitudinal and transverse momentum components at production of the secondaries simulated in the 

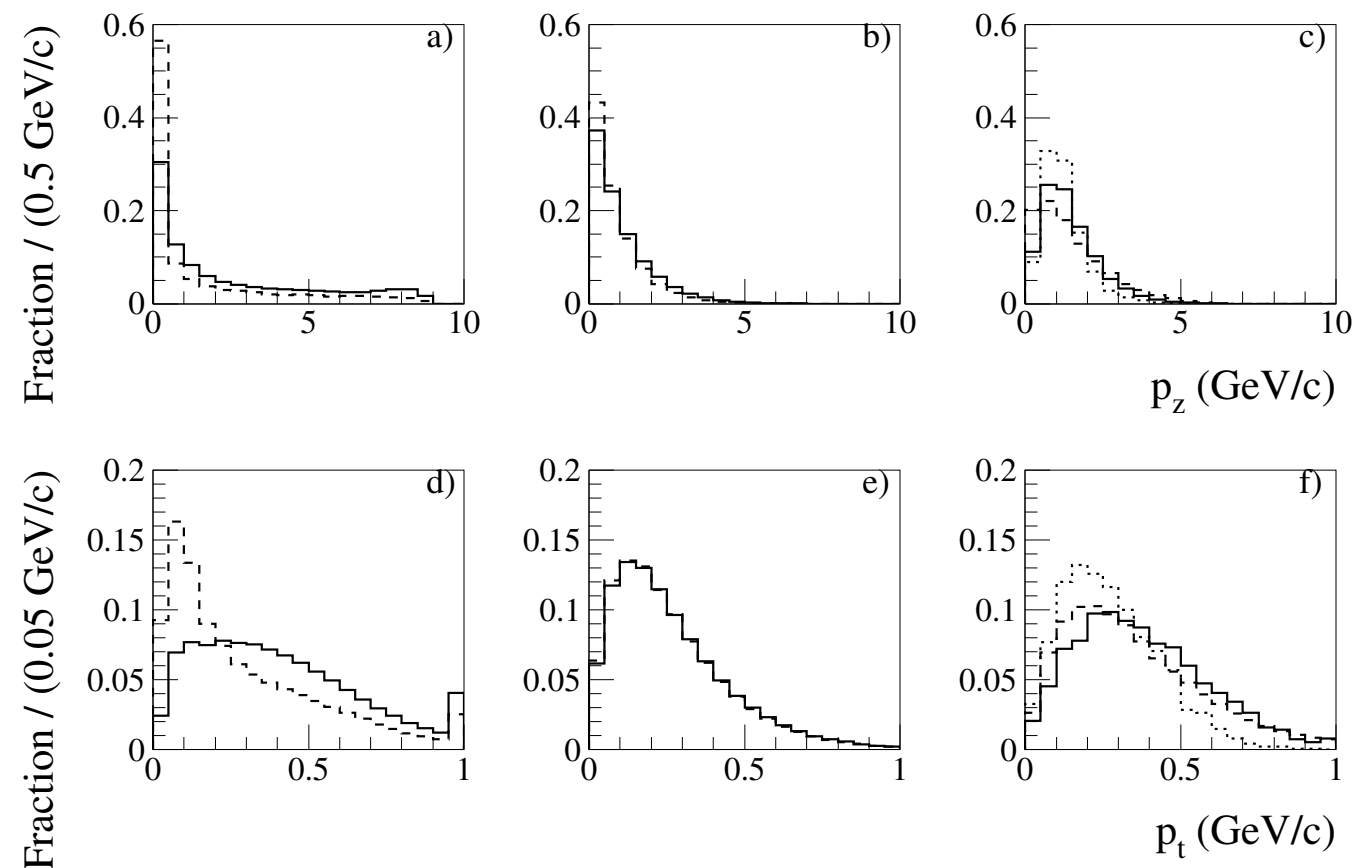

Figure 4.4: One-dimensional projections along the longitudinal (top) and transverse (bottom) momentum components $p_{z}$ and $p_{t}$ of the expected double-differential production crosssections for secondaries produced by $8.9 \mathrm{GeV} / \mathrm{c}$ protons interacting in beryllium. All histograms are normalized to unit area. Panels a) and d) refer to baryon production: solid histograms for protons, dashed for neutrons; b) and e) to non-strange meson production: solid histograms for $\pi^{+}$, dashed for $\pi^{-}$; c) and f) to strange meson production: solid histograms for $K_{L}^{0}$, dashed for $K^{+}$, dotted for $K^{-}$.

interactions of $8.9 \mathrm{GeV} / \mathrm{c}$ protons in beryllium, normalized per unit area. We note here the leading particle effect in the proton and neutron distributions, with momenta extending up to incident proton momentum, and that the pion kinematic distributions at production are peaked at considerably lower momenta compared to the typical momenta for pions yielding neutrinos directed toward the MiniBooNE detector (see Tab. 4.4). 


\subsubsection{Other Inelastic Interactions}

Secondary interactions of pions and protons also play a non-negligible role in determining the MiniBooNE neutrino flux. These particles, once they have been produced in a primary inelastic interaction, can still traverse significant amounts of target and horn material (relative to the inelastic interaction length, see Tab. 4.5), and in turn interact inelastically. Therefore, we describe here the simulation of inelastic interactions of secondary pions and protons of various momenta in beryllium and aluminum.

These inelastic processes, with the exception of $K_{p}>7.5 \mathrm{GeV}$ protons in beryllium discussed in Section 4.4.1, are described by built-in GEANT4 physics models. The final state description model typically used in the simulations is part of the "Low Energy Parametrization Driven Model", based on the GEANT3.21 GHEISHA package [81]. For the purposes of estimating flux systematic uncertainties, alternative built-in physics models have also been explored, namely the "Bertini Intranuclear cascade model", and the "Binary Cascade Model" [81].

Figure 4.5 shows average properties of inelastic interactions of secondary $\pi^{+}$'s and protons in beryllium and aluminum, as a function of the projectile momentum. The average properties shown are the inelastic interaction length $\lambda_{\text {inel }}$, the average number $\left\langle N_{\pi}\right\rangle$ of $\pi^{+}$'s in the inelastic interaction final state, the average momentum $\left\langle p_{\pi}\right\rangle$ for final state $\pi^{+}$'s, and the average angle $\left\langle\theta_{\pi}\right\rangle$ between the incoming projectile and the outgoing final state $\pi^{+}$'s.

Experimental data are available on the total inelastic length, and are shown for comparison in Fig. 4.5. The inelastic interaction lengths for $\pi^{+}$'s and protons are similar, of the order of $40-50 \mathrm{~cm}$. The inelastic cross-sections show a mild momentum dependence over the range $1<p(\mathrm{GeV} / \mathrm{c})<10$.

Pions in the final state tend to be more numerous for higher energy projectiles, for $\pi^{+}$projectiles than for $p$ projectiles, and show little dependence on the target material. The average $\pi^{+}$momenta tend to be much smaller than the projectile momenta, and $\pi^{+}$emission angles are large. 

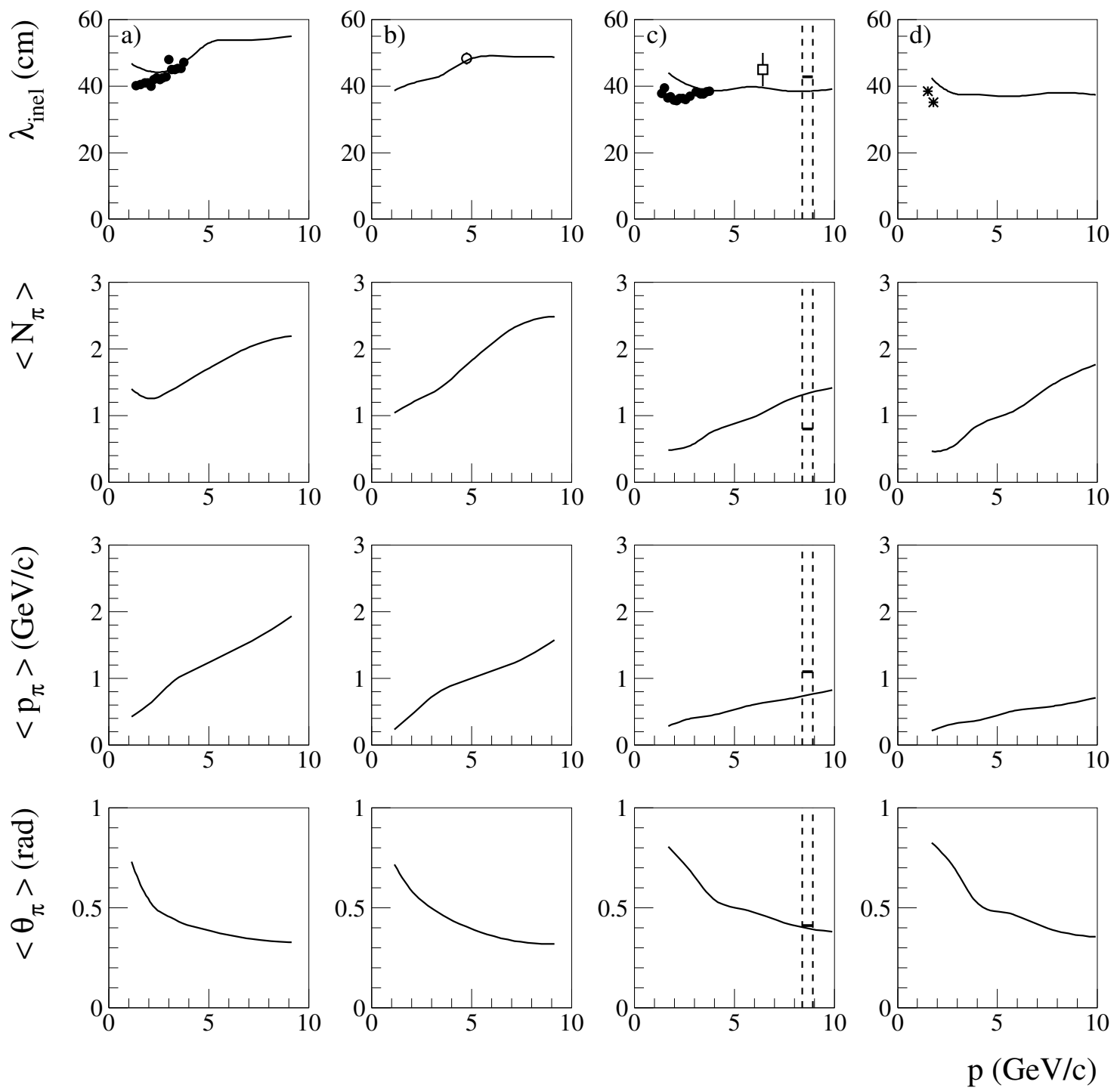

Figure 4.5: Inelastic processes for $\pi^{+}$'s and protons interacting in beryllium and aluminum, as a function of the projectile momentum $p$. From left to right: inelastic $\pi^{+}-B e, \pi^{+}-A l, p$ Be, and p-Al processes. From top to bottom: inelastic interaction length $\lambda_{\text {inel }}$, and average number per inelastic collision $\left\langle N_{\pi}\right\rangle$, momentum $\left\langle p_{\pi}\right\rangle$ and angle with respect to the projectile direction $\left\langle\vartheta_{\pi}\right\rangle$, of final state $\pi^{+}$'s. The curves show the beam Monte Carlo assumptions, the points show experimental data: • from Ref. [93], ○ from [94], $\square$ from [82], * from [95]. Moreover, the values within dashed lines in the p-Be panels show the assumptions adopted for primary protons, described in Section 4.4.1. 

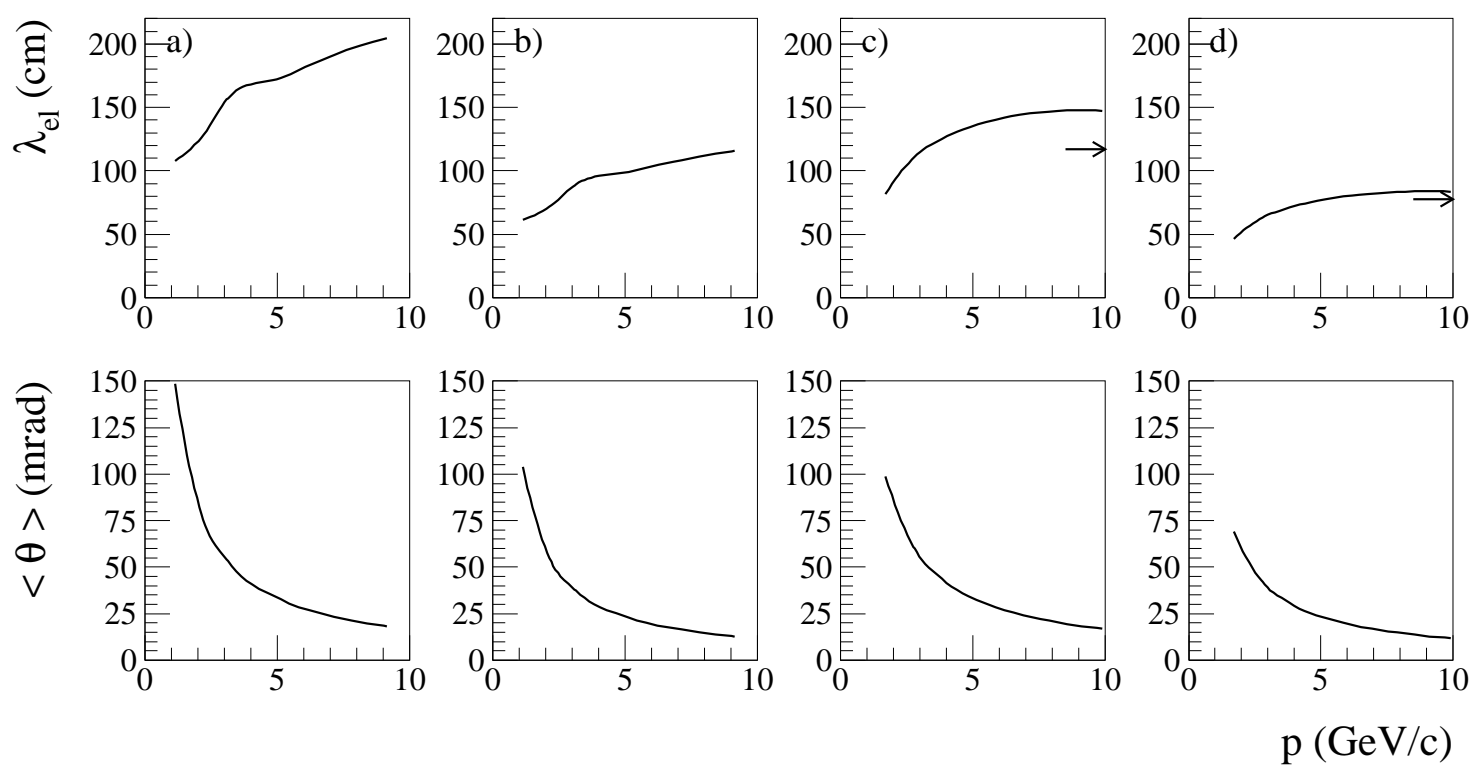

Figure 4.6: Elastic processes for $\pi^{+}$'s and protons interacting in beryllium and aluminum, as a function of projectile momentum $p$. From left to right: elastic $\pi^{+}-B e, \pi^{+}-A l, p$-Be, and p-Al processes. From top to bottom: elastic interaction length $\lambda_{e l}$, and average projectile deflection angle $\langle\vartheta\rangle$. The arrows in the top plots indicate the high-energy values from [60].

\subsubsection{Elastic Interactions}

Apart from scattering inelastically, pions and protons can also interact elastically in the target hall and decay region materials. Unlike the inelastic processes described above, no particle absorption, particle production, or charge exchange are present in this case, and the only effect is an angular deflection of the projectile with respect to its original direction. In Figure 4.6, the elastic interaction length $\lambda_{\mathrm{el}}$ and the average elastic scattering deflection angle assumed by the beam Monte Carlo simulation are shown as a function of projectile momentum.

The elastic interaction length is smaller in aluminum than in beryllium, and similar for protons or pions; moreover, the elastic interaction length tends to increase with projectile momentum, in the $1<p(\mathrm{GeV} / \mathrm{c})<10$ momentum range. Over this range, typical values in beryllium are $110-205 \mathrm{~cm}$, and $45-115 \mathrm{~cm}$ in aluminum. significantly larger than the corresponding inelastic interaction lengths. From the 
data in Ref. [60], and assuming that the total nuclear collision cross-section is given by the sum of the total elastic and inelastic cross-section, the elastic interaction length for $>60 \mathrm{GeV} / \mathrm{c}$ protons can be extracted. The elastic interaction length values from [60] are 117.0 and $77.7 \mathrm{~cm}$ in beryllium and aluminum, respectively, which are also shown in Fig. 4.6.

The mean deflection angles are much smaller in elastic collisions than in the previously discussed inelastic collisions. The angles tend to be larger, on average, for low-energy projectiles, and are very similar for protons and pions. Typical deflection angles in one elastic collision are 10-150 mrad, and are therefore significant.

\subsection{Electromagnetic Processes}

\subsubsection{Particle Trajectories in the MiniBooNE Horn Magnetic Field}

The horn magnetic field provides a large increase in flux: the neutrino rate at MiniBooNE per proton on target has been measured to be about six times larger in horn-on running mode, compared to horn-off running mode (see Chapter 3). Therefore, it is necessary to accurately simulate the motion of charged particles in the field, in order to obtain reliable flux predictions.

The transportation algorithms through a non-uniform magnetic field region used by the beam Monte Carlo simulation have been validated via an independent tracking algorithm. The simple test case considered here is that of a magnetic field region that is similar to the MiniBooNE horn one, with an azimuthal field of magnitude $B=\mu_{0} I /(2 \pi r)$, where $\mu_{0}=4 \pi \cdot 10^{-7} \mathrm{~N} / \mathrm{A}^{2}$ is the permittivity of free space, $I=170$ $\mathrm{kA}$ and $r=\sqrt{x^{2}+y^{2}}$, in the region $2.2 \mathrm{~cm}<r<30 \mathrm{~cm}, 0<z<180 \mathrm{~cm}$, and zero magnetic field otherwise. Positive pions of various momenta, produced at $(x=0, y=$ $0, z=30 \mathrm{~cm})$ and with an initial momentum direction of $p_{x} / p_{z}=1 / 8, p_{y}=0$, are 


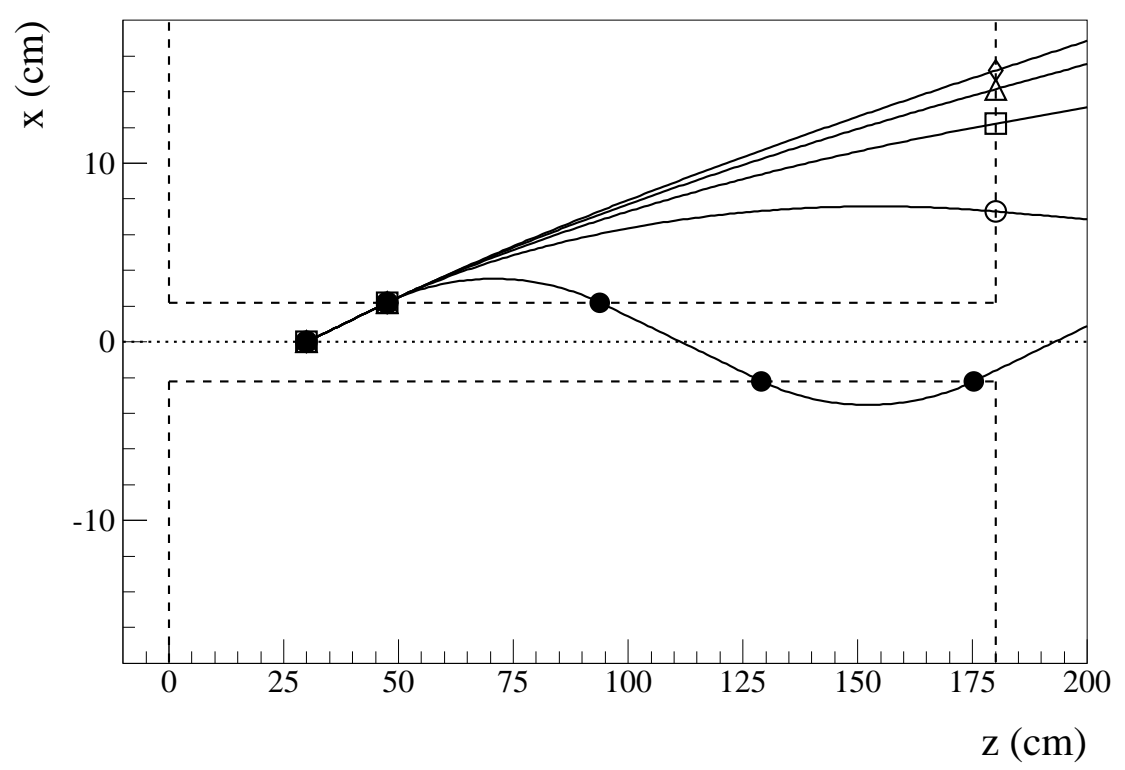

Figure 4.7: Trajectories in the horn magnetic field for 0.5, 1.5, 2.5, 3.5, and 4.5 GeV kinetic energy $\pi^{+}$'s, with initial conditions: $\left(x_{0}=0, z_{0}=30 \mathrm{~cm}, p_{x, 0} / p_{z, 0}=1 / 8\right)$. The points are from a beam Monte Carlo simulation, the dashed curves from the solution of Eq. 4.3 discussed in the text.

tracked through this simple geometry. The particle trajectories are described by the following system of two coupled, second-order differential equations:

$$
\begin{cases}\ddot{x}(t)=\frac{c_{B}}{x(t)} \dot{z}(t), \quad \dot{x}(0)=v_{0} \sin \theta, & x(0)=x_{0} \\ \ddot{z}(t)=-\frac{c_{B}}{x(t)} \dot{x}(t), & \dot{z}(0)=v_{0} \cos \theta, \quad z(0)=z_{0}\end{cases}
$$

where, in SI units:

$$
c_{B}=\frac{\mu_{0} I}{2 \pi} \cdot \frac{q}{m_{\pi} \gamma}
$$

where $\mu_{0}$ and $I$ are defined above, $q$ is the (positive) electron charge, $m_{\pi}$ is the $\pi^{+}$ mass, $\gamma$ is the relativistic $\gamma$ factor for the pion, $v_{0}=c \sqrt{\gamma^{2}-1} / \gamma$, and $\theta=\arctan (1 / 8)$ in this case.

The particle trajectories as calculated by the beam Monte Carlo simulation, and by an independent tracking algorithm based on a Runge-Kutta-Nystrom numerical integration [96] of Eq. 4.3, are shown to be consistent with each other in Fig, 4.7, for 

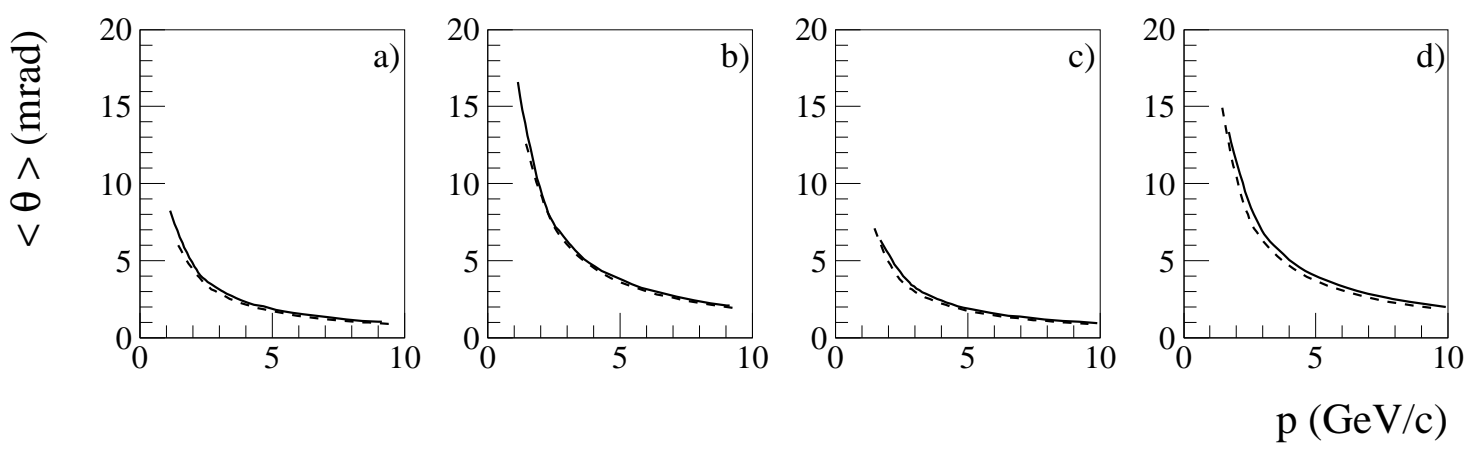

Figure 4.8: Multiple Coulomb scattering results obtained from a simulation of $10 \mathrm{~cm}$ of material, as a function of projectile momentum $p$. From left to right: multiple Coulomb scattering of $\pi^{+}$in $B e, \pi^{+}$in $A l, p$ in Be, $p$ in Al. The average projectile deflection angle, $\langle\theta\rangle$, is shown. The dashed curves, almost indistinguishable from the beam Monte Carlo solid curves, show the predictions from Eqs. 4.5 and 4.6 in the text.

pions of various momenta.

\subsubsection{Multiple Coulomb Scattering}

Any charged particle traversing a material undergoes many small electromagnetic scatters that can be described cumulatively, at a macroscopic level. The beam Monte Carlo simulation uses the Lewis formalism to simulate multiple Coulomb scattering. The multiple Coulomb scattering angle distributions for pions and protons, simulated by the beam Monte Carlo program through $10 \mathrm{~cm}$ of beryllium and aluminum materials, are shown in Fig 4.8 as a function of projectile momenta. This material thickness is chosen in this example because it is comparable to the materials traversed by protons and pions yielding muon neutrinos at MiniBooNE (see Tab. 4.4).

Multiple Coulomb scattering is more important for low-momentum than for high-momentum projectiles, for aluminum than beryllium, and is similar for protons and pions. Typical scattering angles over $10 \mathrm{~cm}$ of material traversed are between 1 and $20 \mathrm{mrad}$.

The beam Monte Carlo results can be confronted with the Highland formula from 
Ref. [60], describing the average multiple Coulomb scattering angle $\langle\theta\rangle$ :

$$
\langle\theta\rangle_{H}=\sqrt{\frac{\pi}{2}} \sigma_{\theta}
$$

where:

$$
\sigma_{\theta}=\frac{13.6 \mathrm{MeV}}{\beta c p} \sqrt{z / X_{0}}\left[1+0.038 \ln \left(z / X_{0}\right)\right]
$$

In Eq 4.6, $\beta c$ is the projectile velocity, $p$ its momentum, $z \simeq 10 \mathrm{~cm}$ is the material traversed, $X_{0}$ is the material radiation length, equal to $35.28 \mathrm{~cm}$ for beryllium and $8.90 \mathrm{~cm}$ for aluminum [60]. The dashed curves in Fig. 4.8 show the average deflection angle due to multiple Coulomb scattering as predicted by the Highland formula of Eqs. 4.5 and 4.6, and good agreement with the beam Monte Carlo simulations is obtained.

\subsubsection{Ionization by Charged Hadrons}

The last electromagnetic process discussed here is energy loss by charged hadrons. In the beam Monte Carlo simulation, this process simulates both the continuous energy loss due to ionization and atomic excitation via the Bethe-Bloch formula, as well as the "discrete" part of the ionization via $\delta$-ray emission. As for multiple Coulomb scattering processes, the macroscopic effects due to energy loss are quantified for particles crossing $z=10 \mathrm{~cm}$ of beryllium and aluminum materials. The results from beam Monte Carlo simulations are shown in Fig. 4.9, In Figure 4.9, we define $\Delta$ as the projectile energy loss across the material thickness:

$$
\Delta \equiv-\int_{0}^{10 \mathrm{~cm}} \frac{d E}{d z} d z
$$

The energy loss results in Fig. 4.9 can be compared with the Bethe-Bloch formula given in [60], for $\pi^{+}$or $p$ projectiles in beryllium (aluminum):

$$
-\frac{d E}{d x}=\rho K z^{2} \frac{Z}{A} \frac{1}{\beta^{2}}\left[\frac{1}{2} \ln \frac{2 m_{e} c^{2} \beta^{2} \gamma^{2} T_{\max }}{I^{2}}-\beta^{2}-\frac{\delta}{2}\right]
$$



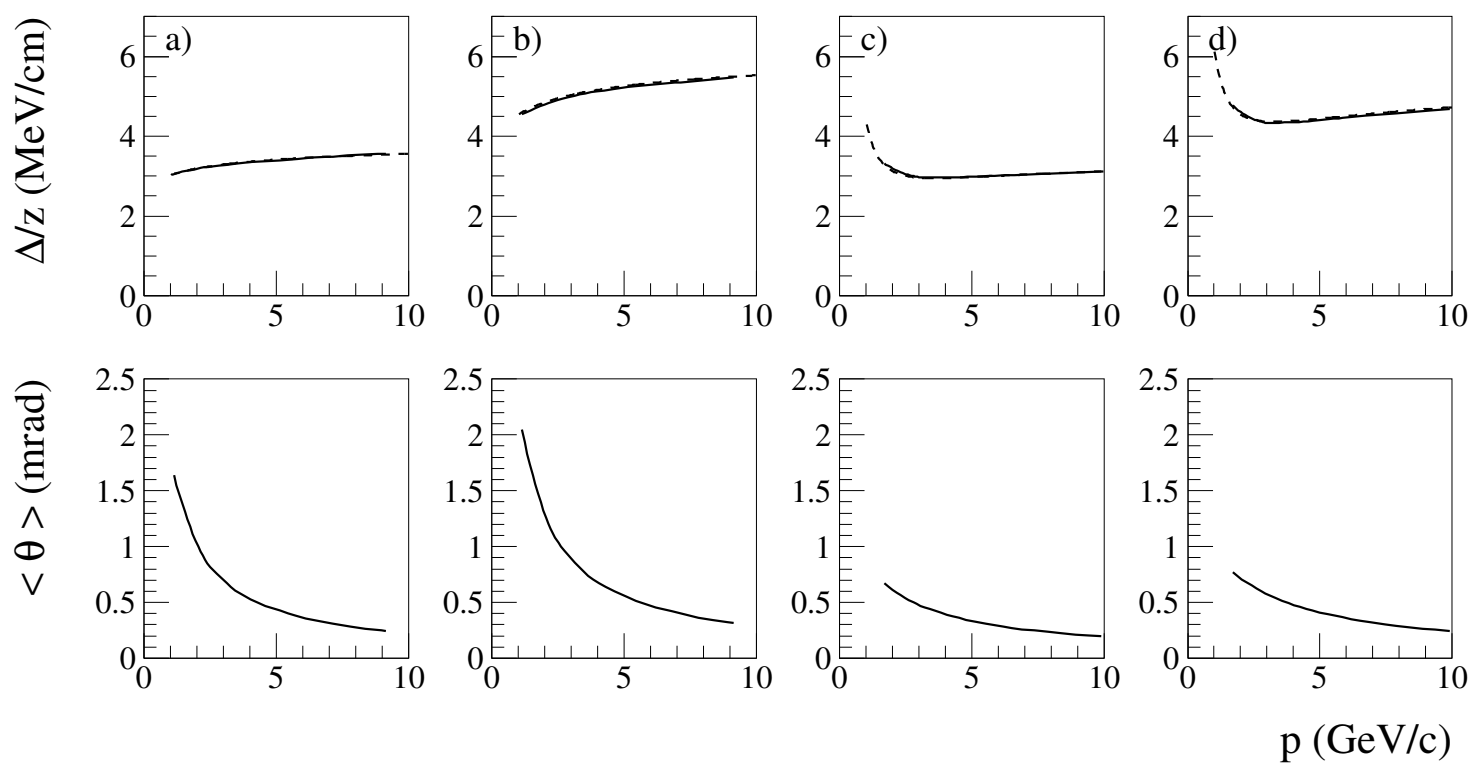

Figure 4.9: Energy loss results obtained from a simulation of $10 \mathrm{~cm}$ of material, as a function of projectile momentum p. From left to right: energy loss of $\pi^{+}$in $B e, \pi^{+}$in $A l, p$ in Be, $p$ in Al. From top to bottom: average energy loss per unit material traversed, $\Delta / z$; average projectile deflection angle, $\langle\theta\rangle$. The dashed curves, almost indistinguishable from the beam Monte Carlo solid curves, show the predictions from Eqs. 4.8 and 4.9 in the text.

where $K=0.307075 \mathrm{MeV} \mathrm{g}^{-1} \mathrm{~mol}^{-1}, z=1$ is the projectile electric charge in units of $e, Z=4(13)$ is the target electric charge in units of $e, A=9.01$ (26.98) is the target atomic weight in units of $\mathrm{g} / \mathrm{mol}, \beta$ is the projectile's velocity in units of $c$, $m_{e} c^{2}=0.511 \mathrm{MeV}$ is the electron mass, $\gamma=\left(1-\beta^{2}\right)^{-1 / 2}, T_{\max }=2 m_{e} c^{2} \beta^{2} \gamma^{2} /(1+$ $\left.2 \gamma m_{e} / M+\left(m_{e} / M\right)^{2}\right)$ is the maximum kinetic energy which can be imparted to a free electron in a single collision, $M=139.57$ or $938.27 \mathrm{MeV} / \mathrm{c}^{2}$ is the projectile's mass, $I=63.7(166.0) \mathrm{eV}$ is the mean excitation energy, and $\delta$ is the density effect correction to ionization energy loss, given by:

$$
\delta= \begin{cases}2(\ln 10) x-\bar{C} & , \text { if } x \geq x_{1} \\ 2(\ln 10) x-\bar{C}+a\left(x_{1}-x\right)^{k} & , \text { if } x_{0} \leq x<x_{1} \\ \delta_{0} 10^{2\left(x-x_{0}\right)} & , \text { if } x<x_{0}\end{cases}
$$


where $x=\log _{10}(p / M c), x_{0}=0.0592(0.1708), x_{1}=1.6922(3.0127), \bar{C}=2.7847$ (4.2395), $a=0.80392$ (0.08024), $k=2.4339$ (3.6345), $\delta_{0}=0.14$ (0.12) [60]. The expected energy loss given by Eqs. 4.8 and 4.9 is consistent with the beam Monte Carlo assumptions.

Figure 4.9 also shows the mean projectile deflection angles after traversing $z=10$ $\mathrm{cm}$ of material. These angles are very small, of the order of $1 \mathrm{mrad}$, and negligible with respect to the deflection angles due to nuclear elastic scattering or multiple Coulomb scattering.

\subsection{Neutrinos from Meson and Muon Decays}

Neutrinos reaching the MiniBooNE detector are produced via the decays of charged pions, kaons, and muons. Particle lifetimes, decay branching ratios, Lorentz boosts, and center-of-mass kinematics of the neutrinos produced in the decays affect the neutrino flux predictions, and are discussed here.

The neutrino parent lifetimes and branching ratios used in the simulation are given in Tab. 4.9, for $\pi^{+}, K^{+}, K_{L}^{0}$, and $\mu^{+}$neutrino parents, and the corresponding decays of negatively-charged particles are also simulated. Given the relevance of decays yielding electron neutrinos for the MiniBooNE $\nu_{\mu} \rightarrow \nu_{e}$ search, the GEANT4 built-in decay channels and branching ratios have been updated with the values in Tab. 4.9 to yield more accurate electron neutrino predictions 97 .

The effect of Lorentz boosts is shown in Fig. 4.10. Figure 4.10a shows the decay

length $\lambda_{\text {decay }}$ as a function of neutrino parent momentum $p$. The data points from beam Monte Carlo simulations match well the $\gamma \beta c \tau$ expectations, where the lifetime $\tau$ for a neutrino parent type is the one given in Tab. 4.9, and $\gamma \beta=p / m$, where $m$ is the neutrino parent mass. Also, Lorentz boosts are applied in the simulation to determine the neutrino kinematics in the laboratory frame, once the neutrino kinematics are generated in the parent rest frame. In Figure $4.10 \mathrm{~b}$, we show the 


\begin{tabular}{|c|r|l|r|}
\hline Particle & $\begin{array}{r}\text { Lifetime } \\
(\mathrm{ns})\end{array}$ & Decay mode & Branching ratio \\
& 26.03 & $\mu^{+} \nu_{\mu}$ & 99.9877 \\
\hline$\pi^{+}$ & & $e^{+} \nu_{e}$ & 0.0123 \\
\hline$K^{+}$ & 12.37 & $\mu^{+} \nu_{\mu}$ & 63.17 \\
& & $\pi^{+} \pi^{0}$ & 21.2 \\
& & $\pi^{+} \pi^{+} \pi^{-}$ & 5.6 \\
& & $\pi^{0} e^{+} \nu_{e}$ & 5.13 \\
& & $\pi^{0} \mu^{+} \nu_{\mu}$ & 3.2 \\
& & $\pi^{+} \pi^{0} \pi^{0}$ & 1.7 \\
\hline \multirow{2}{*}{$K_{L}^{0}$} & \multirow{2}{*}{51.70} & $\pi^{0} \pi^{0} \pi^{0}$ & 19.45 \\
& & $\pi^{-} e^{+} \nu_{e}$ & 20.4 \\
& & $\pi^{+} e^{-} \bar{\nu}_{e}$ & 20.27 \\
& & $\pi^{-} \mu^{+} \nu_{\mu}$ & 13.55 \\
& & $\pi^{+} \mu^{-} \bar{\nu}_{\mu}$ & 13.46 \\
& & $\pi^{0} \pi^{+} \pi^{-}$ & 12.87 \\
\hline \multirow{2}{*}{2197.03} & $e^{+} \nu_{e} \bar{\nu}_{\mu}$ & 100.0 \\
\hline
\end{tabular}

Table 4.9: Particle lifetimes, decay modes and branching ratios used in the beam Monte Carlo simulation.

neutrino energy as a function of the neutrino parent momentum $p$ in the laboratory frame, for $\pi^{+} \rightarrow \mu^{+} \nu_{\mu}$ decays and for neutrinos crossing a disk of radius $R_{\text {det }}=6.1$ $\mathrm{m}$ and located $L=541 \mathrm{~m}$ from the pion production location, that is for the neutrino phase space of relevance to MiniBooNE. The dashed curves in Fig. 4.10b correspond to the maximum and minimum neutrino energies, given by:

$$
\left\{\begin{array}{l}
E_{\nu, \max }=\gamma E_{\nu}^{C M}(1+\beta) \\
E_{\nu, \min }=\gamma E_{\nu}^{C M}\left(1+\beta \cos \theta_{\max }^{C M}\right)
\end{array}\right.
$$

where $E_{\nu}^{C M}=\left(m_{\pi}^{2}-m_{\mu}^{2}\right) /\left(2 m_{\pi}\right)$ is the neutrino energy in the pion rest frame, $\gamma=$ $\sqrt{1+\left(p / m_{\pi}\right)^{2}}, \beta=\sqrt{\gamma^{2}-1} / \gamma, \theta_{\text {max }}^{C M}=2 \arctan \left(\gamma \tan \theta_{\max }\right)$ and $\theta_{\text {max }}=\arctan \left(R_{\mathrm{det}} / L\right)$ 

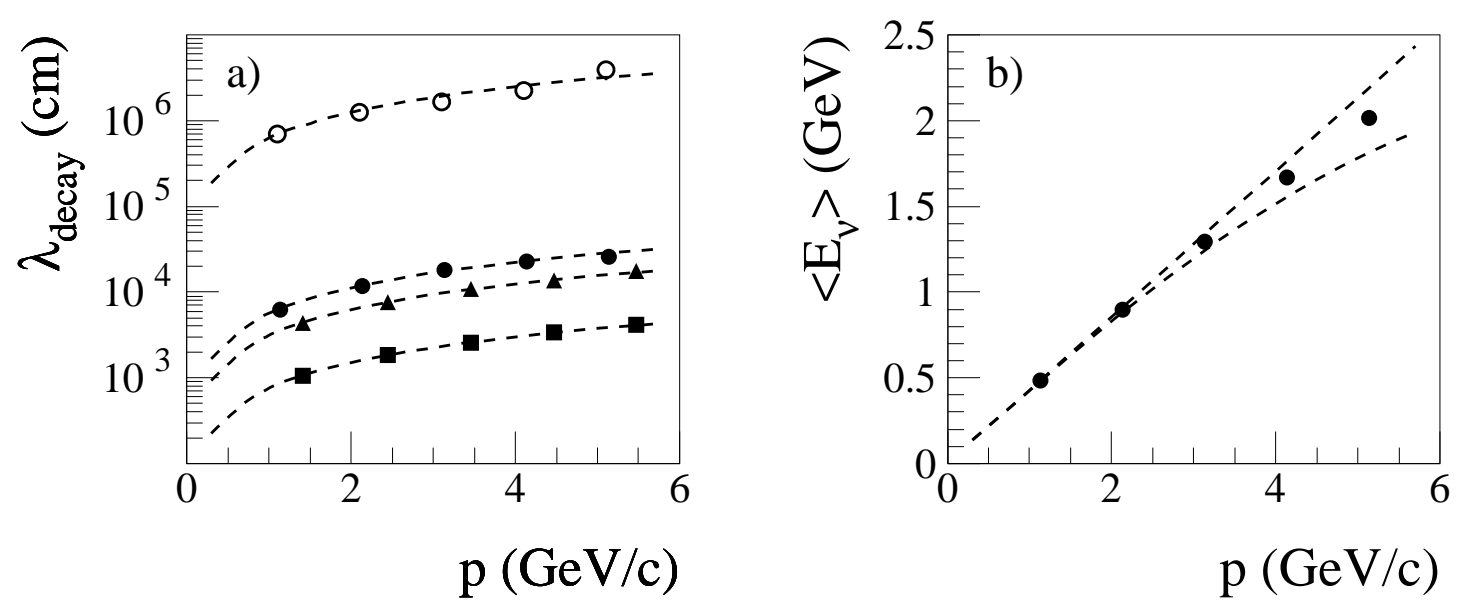

Figure 4.10: Validation of decay routines in the beam Monte Carlo simulation. Fig. 4.10a shows the decay length $\lambda_{\text {decay }}$ as a function of the parent momentum $p$, for (from bottom to top) $K^{+}, \pi^{+}, K_{L}^{0}, \mu^{+}$parents. Fig. 4.10b) shows the average neutrino energy in $\pi^{+} \rightarrow$ $\nu_{\mu} \rightarrow \mu^{+}$decays as a function of pion momentum $p$, for neutrinos crossing the MiniBooNE detector. The points are the results from beam Monte Carlo simulations, the dashed curves are the minimum and maximum neutrino energies discussed in the text.

are the angles between the pion direction and the neutrino directions in the pion rest frame and in the laboratory frame, respectively. From Fig. 4.10 b, one finds that the average neutrino energy predicted by the beam Monte Carlo simulation lies approximately in between the minimum and maximum energies from Eq. 4.10. This is the expected result for the average neutrino energy, given the isotropic neutrino angular distribution in the pion rest frame: in this case, $d N / d \cos \theta^{C M}=$ const in the pion rest frame implies $d N / d E_{\nu}=$ const in the laboratory frame, for fixed pion momenta.

Finally, we discuss the kinematics of neutrino decays in the rest frame of the neutrino parents, for the most relevant decays yielding muon and electron neutrinos, separately. 

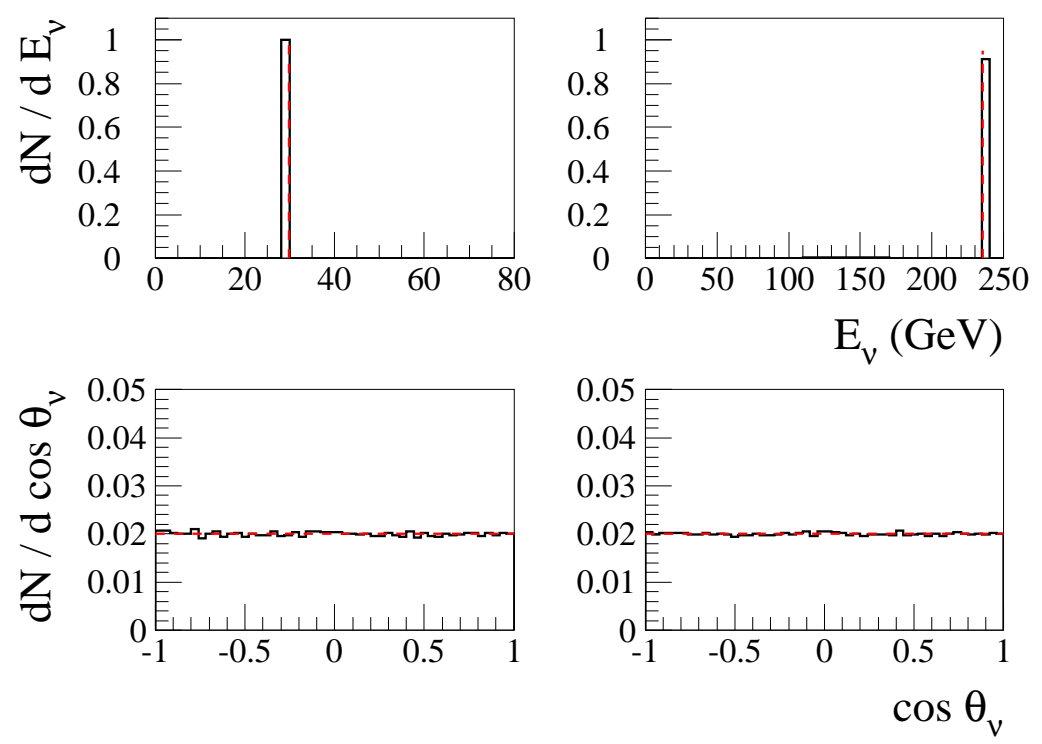

Figure 4.11: Muon neutrino kinematic distributions in the neutrino parent rest frame. The top row shows the neutrino energy distribution, the bottom row shows the neutrino angular distribution with respect to the $z$-direction in the laboratory frame. The left column refers to $\pi^{+}$decays, the right column to $K^{+}$decays. The histograms are the results of beam Monte Carlo simulations, the dashed curves are the expectations discussed in the text.

\subsubsection{Decays into Muon Neutrinos}

Muon neutrinos reaching MiniBooNE are mostly produced by $\pi^{+}$decays, with a nonnegligible contribution from $K^{+}$decays (see Tab. 4.3). The histograms in Fig. 4.11 show the muon neutrino energy and angular distributions in $\pi^{+}$and $K^{+}$decays at rest. The angular distributions are flat, while the neutrino energy from the $\pi^{+} / K^{+} \rightarrow$ $\mu^{+} \nu_{\mu}$ two-body decays is $\left(m_{\pi / K}^{2}-m_{\mu}^{2}\right) /\left(2 m_{\pi / K}\right)$. For kaon decays, a small fraction $(\simeq 5 \%)$ of muon neutrinos are simulated with a continuous energy distribution from $K^{+} \rightarrow \pi^{0} \mu^{+} \nu_{\mu}$ three-body decays (see Tab. 4.9.

\subsubsection{Decays into Electron Neutrinos}

Muon decays and leptonic decays of kaons (both charged and neutral) are predicted to yield almost the entire electron neutrino flux at MiniBooNE (see Tab. 4.3). The 

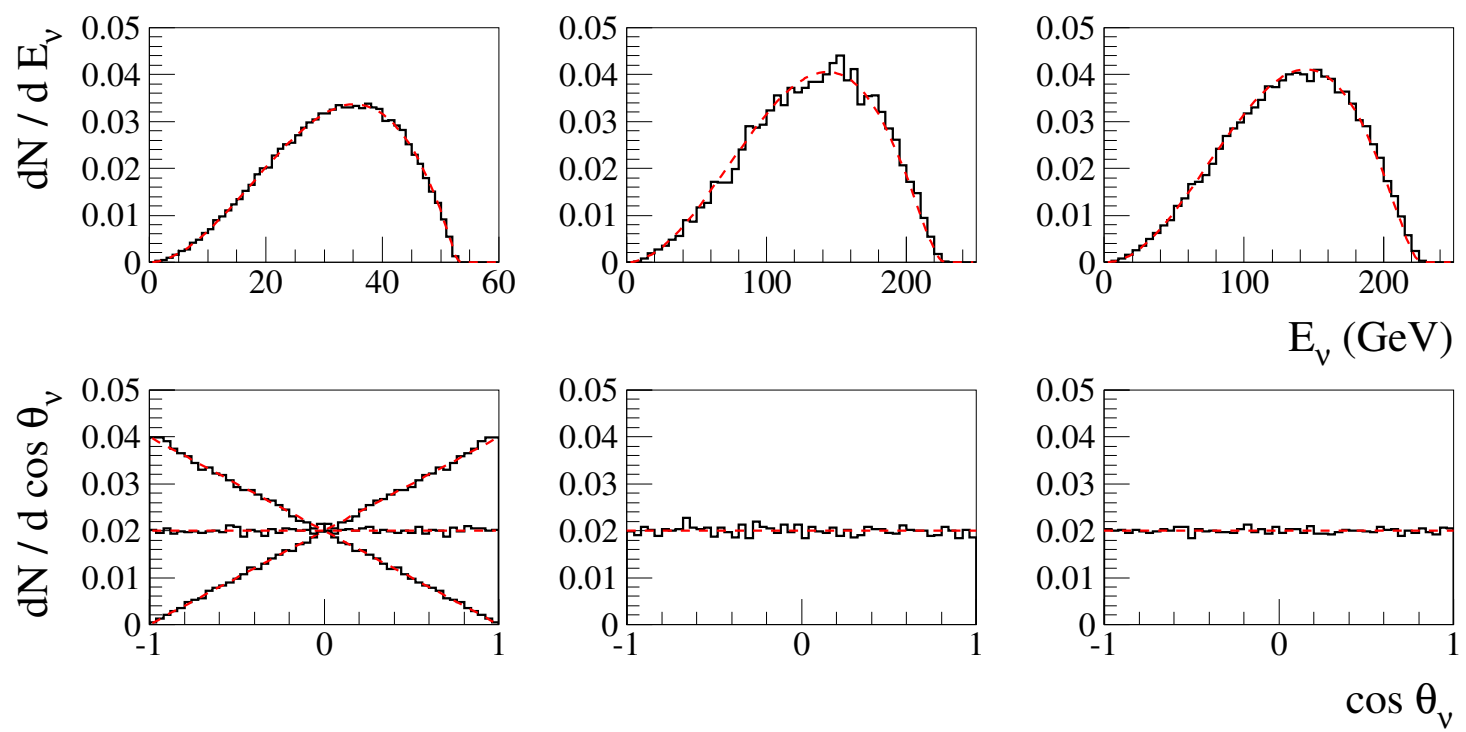

Figure 4.12: Electron neutrino kinematic distributions in the neutrino parent rest frame. The left column refers to $\mu^{+}$decays, the middle column to $K^{+}$decays, and the right column to $K_{L}^{0}$ decays. The top row shows the neutrino energy distribution, the bottom row shows the neutrino angular distribution with respect to the $z$-direction in the lab frame, defined as the beam direction. The three curves for muons correspond to the cases: $P_{z}=-1,0,+1$, where $P_{z}$ is the projection along $z$ of the muon polarization vector in the muon rest frame. The histograms are the results of beam Monte Carlo simulations, the dashed curves are the expectations discussed in the text.

neutrino energy and angular distribution from $\mu^{+}$decays is, neglecting terms proportional to the electron mass [98]:

$$
\frac{d N}{d x d \cos \theta_{\nu}} \propto \frac{12 x^{2}}{4 \pi}(1-x)\left(1 \mp P_{z} \cos \theta\right)
$$

where $\cos \theta_{\nu}$ is the neutrino emission angle with respect to the beam direction $z, P_{z}$ is the projection along $z$ of the muon polarization vector in the muon rest frame, and $x=2 E_{\nu} / m_{\mu}$, with $0<x<1$. In $\pi^{+} \rightarrow \mu^{+} \rightarrow \nu_{e}$ decays, the muon polarization in the muon rest frame is calculated from the known muon polarization in the pion rest frame, and boosting the polarization vector into the muon rest frame.

For the kaon three-body decays yielding electron neutrinos (that is, $K \rightarrow \pi e \nu_{e}$, or $K_{e 3}$ decays), the neutrino angular distribution, in the kaon rest frame and with respect 
to the beam direction, is flat. Assuming that only the vector current contributes to the kaon semileptonic decay matrix element, and neglecting electron mass terms, the neutrino energy distribution in the kaon rest frame is [99]:

$$
\frac{d N}{d E_{\nu}} \propto \int_{E_{e,-}}^{E_{e,+}} d E_{e}\left(2 E_{e} E_{\nu}-m_{k} E_{\pi}^{\prime}\right)\left|f_{+}(t)\right|^{2}
$$

where all quantities refer to the kaon rest frame, $E_{e}$ is the electron energy, $E_{\pi}^{\prime} \equiv$ $E_{\pi}^{\max }-E_{\pi}, E_{\pi}^{\max }=\left(m_{K}^{2}+m_{\pi}^{2}\right) /\left(2 m_{K}\right)$ is the maximum energy that can be transferred to the pion, $E_{\pi}$ is the pion energy, $f_{+}$is a form factor depending only on the square of the four-momentum transfer to the leptons, $t=\left(p_{k}-p_{\pi}\right)^{2}=m_{k}^{2}+m_{\pi}^{2}-2 m_{k} E_{\pi}$, and $E_{e, \pm}$ are integration limits on the electron energy:

$$
\left\{\begin{array}{l}
E_{e,-}=\frac{m_{K}^{2}-m_{\pi}^{2}}{2 m_{k}}-E_{\nu} \\
E_{e,+}=\frac{1}{2}\left(m_{k}-\frac{m_{\pi}^{2}}{m_{k}-2 E_{\nu}}\right)
\end{array}\right.
$$

The beam Monte Carlo simulation also assumes, as customarily done, a linear dependence of the form factor $f_{+}$on $t$ :

$$
f_{+}(t) \propto\left(1+\lambda_{+} t / m_{\pi}^{2}\right)
$$

For $K_{e 3}^{+}\left(K_{e 3}^{0}\right)$ decays, the coefficient $\lambda_{+}$for the linear expansion of the form factor is $0.0277(0.0291)$ [60].

The expected kinematics for electron neutrinos in muon decays and semileptonic kaon decays described above are shown with dashed lines in Fig. 4.12, and agree well with the beam Monte Carlo results.

\subsection{Neutrino Flux Predictions at the MiniBooNE Detector}

In this section we present the results of the beam Monte Carlo simulations, that is the neutrino flux predictions at the MiniBooNE detector location, as a function of 


\begin{tabular}{|c|c|r|r|}
\hline Neutrino Flavor & Neutrino Parent & $\phi\left(\mathrm{cm}^{-2} \mathrm{pot}^{-1}\right)$ & $\left\langle E_{\nu}\right\rangle(\mathrm{GeV})$ \\
\hline all & all & $5.22 \cdot 10^{-10}$ & 0.760 \\
\hline$\nu_{\mu}$ & all & $4.84 \cdot 10^{-10}$ & 0.778 \\
$\nu_{\mu}$ & $\pi^{+}$ & $4.69 \cdot 10^{-10}$ & 0.734 \\
$\nu_{\mu}$ & $K^{+}$ & $1.42 \cdot 10^{-11}$ & 2.250 \\
\hline $\bar{\nu}_{\mu}$ & all & $3.47 \cdot 10^{-11}$ & 0.488 \\
$\bar{\nu}_{\mu}$ & $\pi^{-}$ & $3.24 \cdot 10^{-11}$ & 0.470 \\
\hline$\nu_{e}$ & all & $3.07 \cdot 10^{-12}$ & 0.939 \\
$\nu_{e}$ & $\mu^{+}$ & $1.70 \cdot 10^{-12}$ & 0.665 \\
$\nu_{e}$ & $K^{+}$ & $1.07 \cdot 10^{-12}$ & 1.321 \\
\hline $\bar{\nu}_{e}$ & all & $3.44 \cdot 10^{-13}$ & 0.888 \\
$\bar{\nu}_{e}$ & $K_{L}^{0}$ & $2.55 \cdot 10^{-13}$ & 1.032 \\
\hline
\end{tabular}

Table 4.10: Summary of neutrino flux predictions at MiniBooNE, for neutrino running mode. The fluxes $\phi$, as well as the neutrino energy $\left\langle E_{\nu}\right\rangle$ averaged over the flux distribution, are given. The most important contributions from the various neutrino flavors and neutrino parent types are shown.

neutrino energy and for all relevant neutrino flavors.

Table 4.10 shows a summary on neutrino flux predictions, given in terms of total flux and mean neutrino energy averaged over the flux distributions, for the various neutrino flavors and for the most important neutrino parent types. The overall neutrino flux prediction is $5.22 \cdot 10^{-10} \mathrm{~cm}^{-2}$ pot $^{-1}$, with a mean neutrino energy of $0.76 \mathrm{GeV}$. The flux prediction is $4.84 \cdot 10^{-10} \mathrm{~cm}^{-2}$ pot $^{-1}$ for muon neutrinos, $3.47 \cdot 10^{-11} \mathrm{~cm}^{-2}$ pot $^{-1}$ for muon antineutrinos, $3.07 \cdot 10^{-12} \mathrm{~cm}^{-2}$ pot $^{-1}$ for electron neutrinos, $3.44 \cdot 10^{-13} \mathrm{~cm}^{-2}$ pot $^{-1}$ for electron antineutrinos, yielding a $0.6 \% \nu_{e} / \nu_{\mu}$ flux ratio, and a $0.7 \%\left(\nu_{e}+\bar{\nu}_{e}\right) /\left(\nu_{\mu}+\bar{\nu}_{\mu}\right)$ flux ratio.

Figure 4.13 shows the neutrino energy distributions for muon neutrinos, muon antineutrinos, and electron neutrinos. Figure 4.14 shows the muon neutrino energy 
x $10^{-10}$
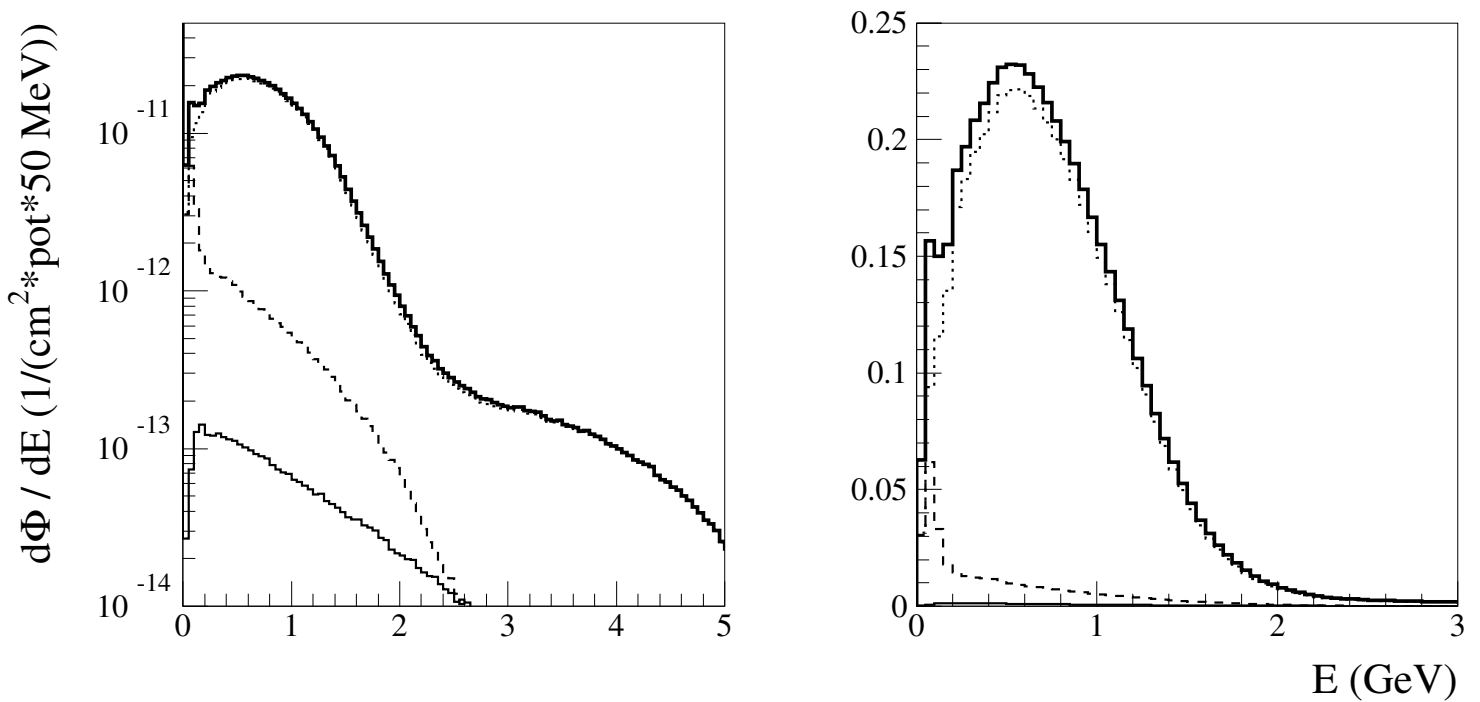

Figure 4.13: Predicted neutrino flux as a function of neutrino energy, for all neutrinos reaching MiniBooNE, in neutrino running mode. The thick solid histogram is for all neutrinos, the thin solid histogram for $\nu_{e}$, the dashed histogram for $\bar{\nu}_{\mu}$, the dotted histogram for $\nu_{\mu}$. The fluxes are shown on a logarithmic scale on the left, and on a linear scale on the right.

distribution, together with the partial contributions from $\pi^{+}$and $K^{+}$decays. Figure 4.15 shows the electron neutrino energy distribution, together with the partial contributions from $\mu^{+}, K^{+}$, and $K_{L}^{0}$ decays. 


$$
\text { x } 10^{-10}
$$
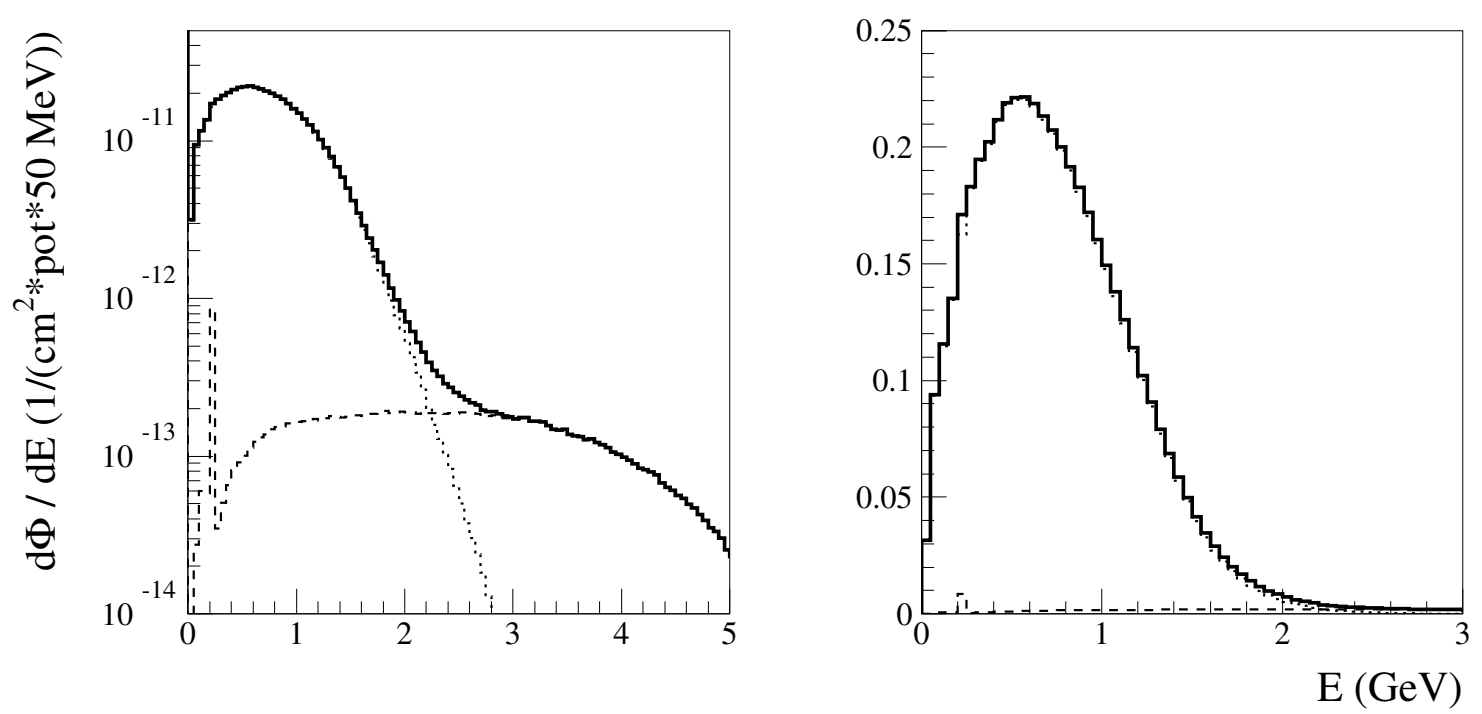

Figure 4.14: Predicted muon neutrino flux as a function of neutrino energy, for all muon neutrinos reaching MiniBooNE, in neutrino running mode. The solid histogram is for all muon neutrinos, the dashed histogram for muon neutrinos from $K^{+}$decay, the dotted histogram for muon neutrinos from $\pi^{+}$decay. The fluxes are shown on a logarithmic scale on the left, and on a linear scale on the right. 
$\mathrm{x} 10^{-12}$
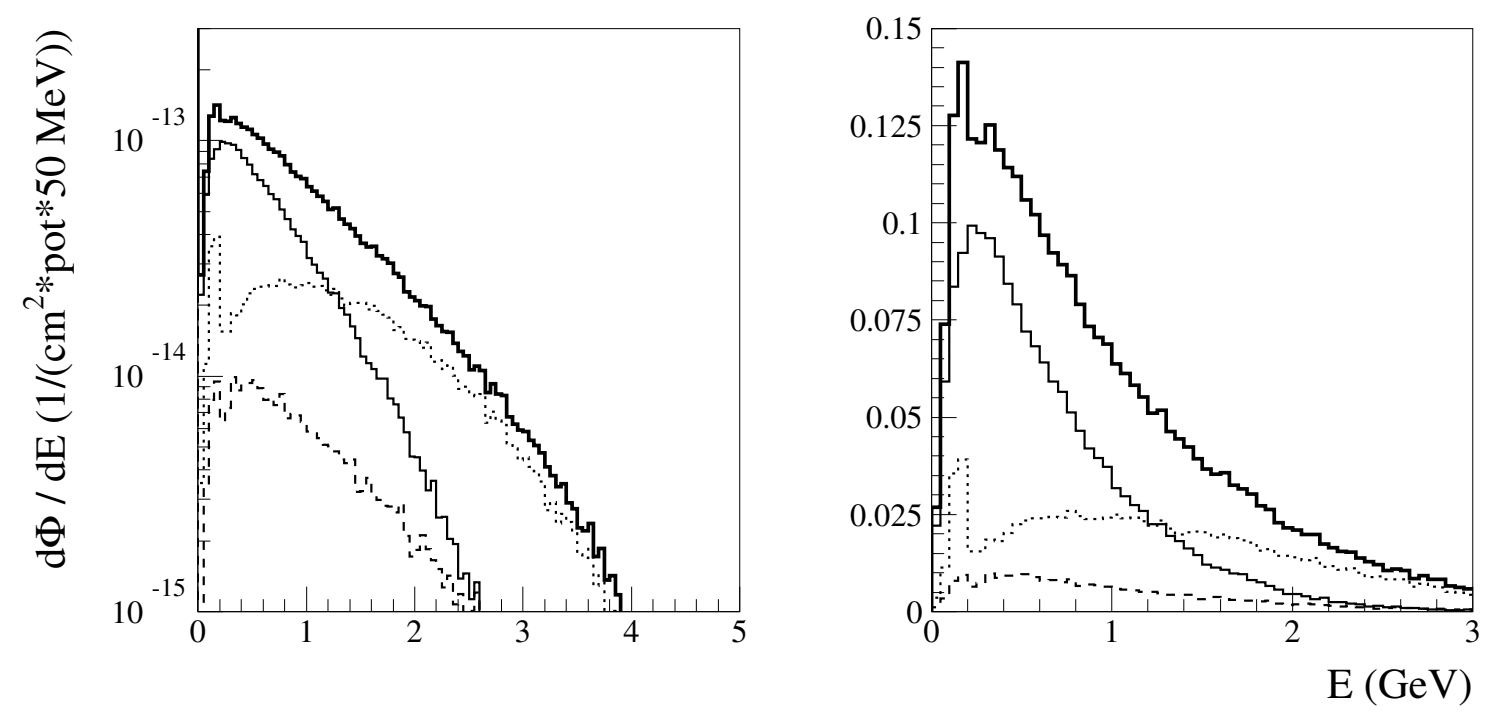

Figure 4.15: Predicted electron neutrino flux as a function of neutrino energy, for all electron neutrinos reaching MiniBooNE, in neutrino running mode. The thick solid histogram is for all electron neutrinos, the thin solid histogram for electron neutrinos from $\mu^{+}$decay, the dashed histogram for electron neutrinos from $K_{L}^{0}$ decay, the dotted histogram for electron neutrinos from $K^{+}$decay. The fluxes are shown on a logarithmic scale on the left, and on a linear scale on the right. 


\section{Chapter 5}

\section{Overview of Neutrino Interactions in the $1 \mathrm{GeV}$ Energy Regime}

We review here the current theoretical and experimental understanding of low-energy neutrino interactions. The description and the predictions of the NUANCE event generator code [100], used in MiniBooNE to implement all neutrino cross-sections, is given as well. The $\nu_{\mu}$ charged-current, quasi-elastic (CCQE) interaction is the only process described in detail, since it is the focus of the MiniBooNE analysis presented in Chapters 7 and 8. Moreover, the discussion will be restricted to $\nu_{\mu}$ interactions only, since they are estimated to account for $97.5 \%$ of all neutrino interactions in the MiniBooNE detector when the neutrino beamline is operated in neutrino mode.

As discussed in Chapter 4, MiniBooNE detects beam-induced neutrino interactions mostly in the $0.3<E_{\nu}(\mathrm{GeV})<1.5$ energy range. At these energies, the dominant process responsible for neutrino interactions is quasi-elastic scattering. On the other hand, the center-of-mass energy in the collision is high enough to have a non-negligible contribution to the total cross-section from production of hadronic resonances from neutrino-nucleus interactions, and to a lesser degree from deep inelastic scattering (DIS) of neutrinos with the quark constituents of nucleons. Unlike quasielastic scattering, the final states for the latter two neutrino interaction processes 


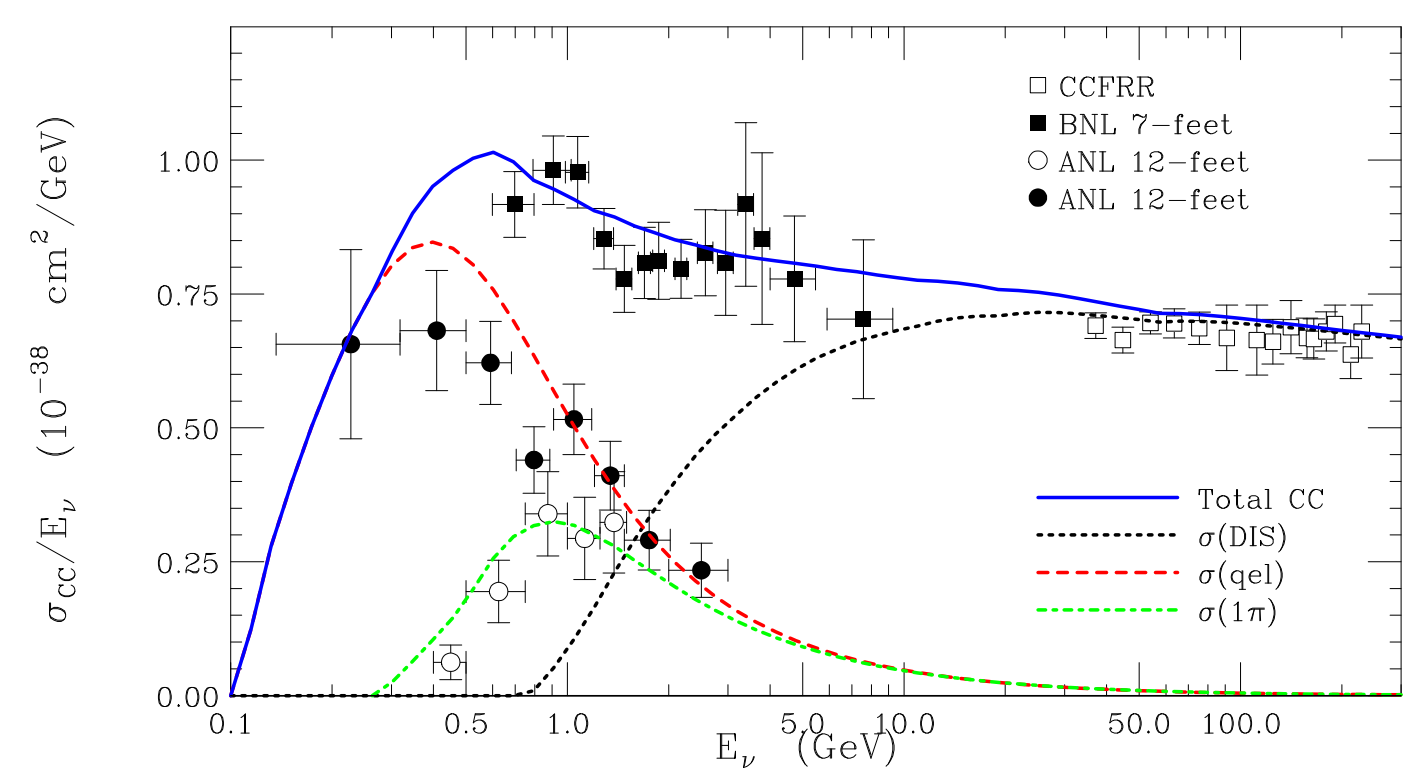

Figure 5.1: Charged-current neutrino cross-section per nucleon divided by neutrino energy, $\sigma_{C C} / E_{\nu}$, over a wide range of neutrino energies $\left(0.1<E_{\nu}(G e V)<300\right)$. The predicted relative contributions from the CCQE (red curve), CC resonant single $\pi$ production (green), and from CC DIS (black) are also shown, together with the total CC cross-section expectation (blue curve) and existing cross-section measurements. The calculations are from [101], while the experimental data are from [102] (solid circles), [103] (empty circles), [104] (solid squares), and [105] (empty squares).

typically include pions, in addition to baryons and leptons.

The individual process contributions to the charged-current part of the total neutrino cross-section are shown in Fig. 5.1, as a function of neutrino energy and divided by neutrino energy. While the quasi-elastic and resonant $\pi$ production (typically from $\Delta \rightarrow N \pi$ ) cross-sections reach an asymptotic value at high energies, the DIS cross-section grows linearly with neutrino energy. 


\subsection{The NUANCE Cross-Section Generator}

In order to obtain reliable cross-section and oscillation measurements at MiniBooNE, a realistic simulation of all relevant neutrino interaction processes is necessary. In MiniBooNE, neutrino cross-sections are simulated via the NUANCE code [100].

NUANCE is a general-purpose neutrino cross-section simulator, modeling virtually all relevant neutrino interaction processes (with varying degrees of sophistication) in the $10^{-1}<E_{\nu}(\mathrm{GeV})<10^{3}$ neutrino energy range. NUANCE is an open source code ${ }^{1}$, originally developed for simulating atmospheric neutrino interactions in the IMB detector. The code has later been greatly generalized, and it is currently used by several neutrino experiments other than MiniBooNE, including Super-Kamiokande, K2K, SNO, and KamLAND. The total neutrino cross-section is computed by summing the contributions from all the simulated exclusive channels: quasi-elastic scattering, neutrino-induced production and decay of baryonic resonances, neutrino-nucleus coherent scattering, and neutrino-electron scattering. The inclusive cross-section contribution from deep inelastic scattering is added to the total cross-section only over a subset of the available hadronic final state phase space (invariant mass of the hadronic system $W$ greater than $2 \mathrm{GeV}$ ), in order to avoid double-counting of resonant contributions. For all processes, both charged-current and neutral-current reactions are simulated. Overall, NUANCE simulates 99 different neutrino interactions, taking also into account the complications arising from nuclear effects. The version of the code that we describe below, v3.000, reflects what is used in this analysis.

Charged-current, quasi-elastic (CCQE) scattering $\nu_{\mu} n \rightarrow \mu^{-} p$ is treated according to the Llewellyn Smith formalism [108, which is described in detail below. The cross-section for neutral-current, quasi-elastic scattering $\nu_{\mu} N \rightarrow \nu_{\mu} N$ is related to the CCQE scattering cross-section, since in electroweak theory the form factors parametrizing the neutral-current process can be fully specified in terms of

\footnotetext{
${ }^{1}$ At the time of writing, the code is available at: http://nuint.ps.uci.edu/nuance/.
} 
the charged-current form factors [109].

The production of baryonic resonances, and their subsequent decay into hadronic final states, is simulated in NUANCE according to the Rein and Sehgal model [110]. The amplitude of each hadronic final state due to a resonance decay, typically composed of one nucleon and one pion, is given by the sum of several contributions arising from various resonances in the $1.08 \mathrm{GeV}<W(N \pi) \equiv\left(p_{N}+p_{\pi}\right)^{2}<2.0 \mathrm{GeV}$ hadronic invariant mass range. The production and decay of each resonance is fully specified by its orbital angular momentum, isospin, and spin quantum numbers, plus the resonance mass central value, width, and inelasticity. In the energy regime of interest to MiniBooNE, the dominant $\Delta$ resonance is $L_{2 i, 2 j}$ (mass) $=P_{3,3}(1232)$, but the effect of eighteen resonances (including interference effects among overlapping resonances) is simulated by NUANCE2, The matrix elements for the production and decay of the resonances are computed using the relativistic quark model of Feynman, Kislinger and Ravndal [111]. The FKR model views the nucleon as the ground state of a three quark system tied together by harmonic forces, whose excitations correspond to baryonic resonances. The nucleon form factors for resonance production are similar to the ones used in quasi-elastic scattering, which we also discuss below. The NUANCE description of neutrino-induced production of resonances include decays to final states with multiple mesons, as well as final states where the $\Delta$ resonance decays radiatively $(\Delta \rightarrow N \gamma)$, an important background to the MiniBooNE $\nu_{\mu} \rightarrow \nu_{e}$ search.

Coherent production of mesons in neutrino interactions are also simulated by NUANCE. Coherent interactions refer to neutrinos interacting with the target nucleus as a whole, with no charge or isospin transfer to the nucleus, no nucleus break-up and very little nuclear recoil energy. Although these processes are responsible for only a small fraction of the total neutrino interactions at MiniBooNE energies, they are unique in that they typically produce a forward-going lepton and a forward-going

\footnotetext{
${ }^{2}$ NUANCE simulates the same resonances included in the original Rein and Sehgal computation, but using a more recent mass spectrum for the resonances.
} 
pion in the final state. Pion production in coherent neutrino-nucleus interactions in NUANCE follows the Rein and Sehgal calculation [112].

Deep inelastic scattering of neutrinos with parton constituents of the nucleons is also taken into account, although its effect is negligible at MiniBooNE energies. The code implements state-of-the-art parton distribution functions from the BodekYang duality-inspired model [113, 114]. Tree-level, neutrino-electron scattering is also included in NUANCE, despite its negligible contribution to the total neutrino crosssection in MiniBooNE.

At MiniBooNE, neutrinos interact with mineral oil, made of Carbon and Hydrogen nuclei. For neutrino reactions on the target nucleons bound in Carbon nuclei, nuclear effects affecting the cross-sections for the individual processes, as well as the final state composition and kinematics, are also simulated by NUANCE. Nuclear effects on charged-current, quasi-elastic neutrino interactions will be discussed below; the same formalism and physics input applies to the other neutrino reactions on bound nucleons simulated by NUANCE.

The MiniBooNE NUANCE cross-section generator allows for a quantitative estimate of the various $\nu_{\mu}$ cross-section contributions, given the MiniBooNE flux discussed in Chapter 4 and for a $\mathrm{CH}_{2}$ nuclear target (a good approximation to mineral oil, as far as neutrino cross-sections are concerned). The various process contributions are shown in Tab. 5.1, split into charged-current (CC) and neutral-current (NC) processes. Charged-current processes are responsible for about $71 \%$ of all $\nu_{\mu}$ interactions at MiniBooNE. The CCQE fraction is the dominant one, corresponding to about $40 \%$ of all $\nu_{\mu}$ interactions at MiniBooNE, followed in importance by CC resonant $\pi$ production, representing $26 \%$ of the total. The fractions given in Tab. 5.1 do not include final state interactions.

We now discuss in more detail the physics assumptions and the experimental validation of the NUANCE CCQE cross-section prediction on free neutrons, and nuclear effects. 


\begin{tabular}{|c|c|r|}
\hline \multicolumn{2}{|c|}{ Interaction Type } & Fraction \\
\multicolumn{1}{|c|}{} & $(\%)$ \\
\hline Quasi-Elastic & $\mathrm{CC}: \nu_{\mu} n \rightarrow \mu^{-} p$ & 39.9 \\
& $\mathrm{NC}: \nu_{\mu} N \rightarrow \nu_{\mu} N$ & 16.3 \\
& $\mathrm{CC} / \mathrm{NC}$ & 56.2 \\
\hline Resonant single $\pi$ & $\mathrm{CC}: \nu_{\mu} N \rightarrow \mu^{-} N \pi$ & 26.4 \\
& $\mathrm{NC}: \nu_{\mu} N \rightarrow \nu_{\mu} N \pi$ & 9.3 \\
& $\mathrm{CC} / \mathrm{NC}$ & 35.7 \\
\hline Coherent single $\pi$ & $\mathrm{CC}: \nu_{\mu} A \rightarrow \mu^{-} \pi^{+} A$ & 2.5 \\
& $\mathrm{NC}: \nu_{\mu} A \rightarrow \nu_{\mu} \pi^{0} A$ & 1.5 \\
& $\mathrm{CC} / \mathrm{NC}$ & 4.0 \\
\hline Resonant multi $\pi$ & $\mathrm{CC}: \nu_{\mu} N \rightarrow \mu^{-} \Delta \pi, \mu^{-} N(\rho / \eta), \mu^{-}(\Lambda / \Sigma) K, \mu^{-} N \pi \pi$ & 2.0 \\
& $\mathrm{NC}: \nu_{\mu} N \rightarrow \nu_{\mu} \Delta \pi, \nu_{\mu} N(\rho / \eta), \nu_{\mu}(\Lambda / \Sigma) K, \nu_{\mu} N \pi \pi$ & 0.8 \\
& $\mathrm{CC} / \mathrm{NC}$ & 2.8 \\
\hline \multirow{2}{*}{ DIS } & $\mathrm{CC}: \nu_{\mu} N \rightarrow \mu^{-} X$ & 0.8 \\
& $\mathrm{NC}: \nu_{\mu} N \rightarrow \nu_{\mu} X$ & 0.3 \\
& $\mathrm{CC} / \mathrm{NC}$ & 1.1 \\
\hline \multirow{2}{*}{ radiative decay } & $\mathrm{CC}: \nu_{\mu} N \rightarrow \mu^{-} N \gamma$ & $<0.1$ \\
& $\mathrm{NC}: \nu_{\mu} N \rightarrow \nu_{\mu} N \gamma$ & 0.1 \\
& $\mathrm{CC} / \mathrm{NC}$ & 0.1 \\
\hline
\end{tabular}

Table 5.1: Event rate fractions for $\nu_{\mu}$ interactions on $\mathrm{CH}_{2}$, assuming the MiniBooNE neutrino flux and neglecting final state interactions. The fractions are split into process types, as well as in charged-current (CC) and neutral-current (NC) processes.

\subsection{The Free Nucleon Quasi-Elastic Interaction}

The charged-current, quasi-elastic (CCQE) neutrino interaction converts the neutrino into the associated charged lepton via the exchange a virtual massive boson $W$ with the target nucleon. For an incoming muon neutrino, the associated charged lepton is a $\mu^{-}$:

$$
\nu_{\mu}\left(p_{1}, \sigma_{1}\right)+n\left(p_{2}, \sigma_{2}\right) \rightarrow \mu^{-}\left(p_{3}, \sigma_{3}\right)+p\left(p_{4}, \sigma_{4}\right)
$$


In Eq 5.1 , the labels $p$ and $\sigma$ denote the energy/momentum of the incoming an outgoing particles, and $\sigma$ the spin states.

Neglecting the neutrino mass and taking $m_{n} \simeq m_{p} \equiv m_{N}$, the differential CCQE cross-section can be expressed in the lab frame as:

$$
\frac{d \sigma}{d Q^{2}}=\frac{\left\langle|M|^{2}\right\rangle}{64 \pi(\hbar c)^{4} m_{N}^{2} E_{\nu}^{2}}
$$

where $Q^{2} \equiv-q^{2}, q \equiv p_{1}-p_{3}$ is the four-momentum carried by the $W,\left\langle|M|^{2}\right\rangle$ is the matrix element squared for the CCQE process, averaged/summed over initial/final spin states (since the particle's polarization is generally not measured), and $E_{\nu}$ is the neutrino energy in the lab frame.

For $Q^{2} \ll m_{W}^{2}$, the matrix element can be expressed in terms of the Fermi constant $G_{F}$, and the weak currents associated with the lepton $\left(j_{l}^{\mu}\right)$ and hadronic $\left(j_{h}^{\mu}\right)$ vertices:

$$
M=\frac{G_{F}}{\sqrt{2}} j_{l}^{\mu} j_{h}^{\nu} g_{\mu \nu}
$$

Empirically, the lepton current is shown to have the pure (V-A), parity-violating, form:

$$
j_{l}^{\mu}=\bar{u}_{3} \gamma^{\mu}\left(1-\gamma^{5}\right) u_{1}
$$

where the $u$ 's are Dirac spinors. The hadronic weak current is more complicated, due to the nucleon structure which is parametrized via form factors. The most general form factor decomposition of the weak hadronic current takes the following form, which includes both a vector (V) and an axial (A) parts [106]:

$$
\begin{array}{r}
j_{h}^{\mu}=j_{h, V}^{\mu}-j_{h, A}^{\mu} \\
j_{h, V}^{\mu}=V_{u d} \bar{u}_{4}\left[f_{1}\left(q^{2}\right) \gamma^{\mu}+i \frac{f_{2}\left(q^{2}\right)}{2 m_{N}} \sigma^{\mu \nu} q_{\nu}+f_{3}\left(q^{2}\right) q^{\mu}\right] u_{2} \\
j_{h, A}^{\mu}=V_{u d} \bar{u}_{4}\left[g_{1}\left(q^{2}\right) \gamma^{\mu} \gamma^{5}+i \frac{g_{2}\left(q^{2}\right)}{2 m_{N}} \sigma^{\mu \nu} \gamma^{5} q_{\nu}+g_{3}\left(q^{2}\right) \gamma^{5} q^{\mu}\right] u_{2}
\end{array}
$$

where $V_{u d}$ is the CKM matrix element associated with the vertex $(d W u)$, and the six functions $f$ and $g$ are weak form factors. Equation 5.5 represents the most general form factor decomposition of the weak hadronic current since it requires only Lorentz 
invariance, but not gauge invariance (i.e. current conservation). Lorentz invariance ensures that the form factors can depend only on $q^{2}$ (or alternatively on $l^{2}$, where $l \equiv p_{1}+p_{3}$, since $l \cdot q=m_{\mu}^{2}$ and $\left.l^{2}+q^{2}=2 m_{\mu}^{2}\right)$. The vector current form factors $f_{1}, f_{2}$, $f_{3}$ are known as vector, weak magnetism, and induced scalar form factors, respectively. The axial vector current form factors $g_{1}, g_{2}, g_{3}$ are the axial-vector, pseudotensor, and induced pseudoscalar form factors, respectively [106]. In the absence of strong interactions, only $f_{1}$ and $g_{1}$ would be present.

It is customary to make a number of assumptions to simplify the hadronic weak current parametrization in Eq. 5.5. First, since the electromagnetic current is conserved, it is assumed that also the weak vector current is conserved (conserved vector current, or CVC, hypothesis). The CVC hypothesis is consistent with current experimental knowledge, and implies a null induced scalar form factor: $f_{3}=0$ [106, 107]. Second, G-invariance arguments for the strong interaction and experimental limits on second-class currents are consistent with a null induced pseudotensor form factor: $g_{2}=0$ [106, 107]. Third, it is believed that the contribution of the induced scalar form factor $g_{3}$ to the CCQE cross-section is negligible, and $g_{3}=0$ is generally assumed [108]. We are therefore left with the $f_{1}, f_{2}$, and $g_{1}$ form factors.

Using Dirac algebra, the CCQE differential cross-section in Eq. 5.2 can be reorganized in power series of $(s-u)$, where the Mandelstam variables $s$ and $u$ are defined in the usual way $\left(s=\left(p_{1}+p_{2}\right)^{2}, u=\left(p_{1}-p_{4}\right)^{2}\right.$, and therefore $(s-u) \simeq 4 m_{N} E_{\nu}-Q^{2}$ in the lab frame):

$$
\frac{d \sigma}{d Q^{2}}=\frac{m_{N}^{2} G_{F}^{2}\left|V_{u d}\right|^{2}}{8 \pi(\hbar c)^{4} E_{\nu}^{2}}\left[A\left(Q^{2}\right) \pm B\left(Q^{2}\right) \frac{(s-u)}{m_{N}^{2}}+\frac{C\left(Q^{2}\right)(s-u)^{2}}{m_{N}^{4}}\right]
$$

where the positive (negative) sign refers to neutrinos (antineutrinos), and the explicit dependence of the $A, B$, and $C$ terms from the form factors, expressed in powers of $Q^{2} / m_{N}^{2}$, is given by [108]:

$$
\begin{array}{r}
A\left(Q^{2}\right)=\left(\left|g_{1}\right|^{2}-\left|f_{1}\right|^{2}\right) \frac{Q^{2}}{m_{N}^{2}}+\frac{1}{4}\left[\left|f_{1}\right|^{2}+4 \operatorname{Re}\left[f_{1} f_{2}^{*}\right]+\left|f_{2}\right|^{2}+\left|g_{1}\right|^{2}\right]\left(\frac{Q^{2}}{m_{N}^{2}}\right)^{2}- \\
-\frac{1}{16}\left|f_{2}\right|^{2}\left(\frac{Q^{2}}{m_{N}^{2}}\right)^{3}
\end{array}
$$




$$
\begin{gathered}
B\left(Q^{2}\right)=\operatorname{Re}\left[g_{1}^{*}\left(f_{1}+f_{2}\right)\right] \frac{Q^{2}}{m_{N}^{2}} \\
C\left(Q^{2}\right)=\frac{1}{4}\left(\left|f_{1}\right|^{2}+\left|g_{1}\right|^{2}\right)+\frac{1}{16}\left|f_{2}\right|^{2} \frac{Q^{2}}{m_{N}^{2}}
\end{gathered}
$$

In order to fully specify the CCQE cross-section, the $Q^{2}$ dependence of the form factors and their values at $Q^{2}=0$ need to be specified. We start by the $Q^{2}=0$ values of the form factors. The CVC hypothesis allows to relate the vector and weak magnetism form factors $f_{1}$ and $f_{2}$ to the electromagnetic form factors $F_{1}$ and $F_{2}$ of the proton and nucleon [106, 107]:

$$
\begin{aligned}
& f_{1}\left(Q^{2}\right)=F_{1}^{p}\left(Q^{2}\right)-F_{1}^{n}\left(Q^{2}\right) \\
& f_{2}\left(Q^{2}\right)=F_{2}^{p}\left(Q^{2}\right)-F_{2}^{n}\left(Q^{2}\right)
\end{aligned}
$$

The electromagnetic form factors $F_{1}^{p, n}, F_{2}^{p, n}$ can be related to measured electromagnetic properties of the nucleons in the non-relativistic limit $Q^{2} \rightarrow 0$ [115, 116]:

$$
\begin{array}{r}
F_{1}^{N}\left(Q^{2}=0\right)=Q_{N} \\
\frac{1}{2 m_{N}}\left[F_{1}\left(Q^{2}=0\right)+F_{2}\left(Q^{2}=0\right)\right]=\mu
\end{array}
$$

where $Q_{N}$ is the nucleon charge (in units of $e$ ) and $\mu$ is the magnetic moment (in units of $\left.\mu_{N} \equiv \frac{e}{2 m_{N}}\right)$. On the other hand, the value for $g_{1}\left(Q^{2}=0\right)$ can be found experimentally from nuclear $\beta$ decays [106, 117]. We obtain for the $Q^{2}=0$ values of the weak form factors $f_{1}, f_{2}$, and $g_{1}$ :

$$
\begin{array}{r}
f_{1}\left(Q^{2}=0\right)=1 \\
f_{2}\left(Q^{2}=0\right)=\mu_{p}-\mu_{n}-1 \simeq 3.7059 \\
g_{1}\left(Q^{2}=0\right) \simeq 1.267
\end{array}
$$

We now discuss the $Q^{2}$-dependence of the form factors. Elastic electron-nucleon scattering experiments at $Q^{2} \neq 0$ probe the $Q^{2}$ dependence of the electromagnetic form factors $F_{1}^{p}$ and $F_{2}^{p, n}$, and therefore of the weak form factors $f_{1}$ and $f_{2}$ (by the CVC hypothesis). From the experimental point of view, the following linear combinations 
(known as Sachs electric and magnetic form factors) of the electromagnetic form factors $F_{1}^{p}, F_{2}^{p, n}$ are best measured [107]:

$$
\begin{array}{r}
G_{E}^{N} \equiv F_{1}^{N}-\frac{Q^{2}}{4 m_{N}^{2}} F_{2}^{N} \\
G_{M}^{N} \equiv F_{1}^{N}+F_{2}^{N}
\end{array}
$$

where $N=p, n$. Experimental results are roughly consistent with having the same "dipole" form for the $Q^{2}$ dependence of $G_{E}^{p}, G_{M}^{p}$, and $G_{M}^{n}$ [107]:

$$
\frac{G_{E}^{p}\left(Q^{2}\right)}{G_{E}^{p}\left(Q^{2}=0\right)}=\frac{G_{M}^{p}\left(Q^{2}\right)}{G_{M}^{p}\left(Q^{2}=0\right)}=\frac{G_{E}^{n}\left(Q^{2}\right)}{G_{E}^{n}\left(Q^{2}=0\right)}=\left(1+\frac{Q^{2}}{m_{V}^{2}}\right)^{-2}
$$

Furthermore, one finds that $G_{E}^{n}\left(Q^{2}\right)$ is consistent with zero at all $Q^{2}$. Therefore, the weak vector form factors $f_{1}$ and $f_{2}$ are parametrized in terms of a single parameter $m_{V}$, known as the vector mass:

$$
\begin{aligned}
f_{1}\left(Q^{2}\right) & =\frac{1+\left(1+\mu_{p}-\mu_{n}\right) Q^{2} /\left(4 m_{N}^{2}\right)}{\left(1+Q^{2} /\left(4 m_{N}^{2}\right)\right)\left(1+Q^{2} / m_{V}^{2}\right)^{2}} \\
f_{2}\left(Q^{2}\right) & =\frac{\mu_{p}-\mu_{n}}{\left(1+Q^{2} /\left(4 m_{N}^{2}\right)\right)\left(1+Q^{2} / m_{V}^{2}\right)}
\end{aligned}
$$

In analogy with the vector form factors, the axial vector form factor is presumed to obey a similar $Q^{2}$ dependence, parametrized in terms of a second parameter $m_{A}$ known as the axial mass [107]:

$$
g_{1}\left(Q^{2}\right) \simeq \frac{1.267}{\left(1+Q^{2} / m_{A}^{2}\right)^{2}}
$$

Global fits to elastic electron-nucleon scattering data suggest the value of 0.84 $\mathrm{GeV}$ for the vector mass, $m_{V}$. On the other hand, the axial form factor can only be extracted from neutrino scattering; analysis of current neutrino data suggest the value $m_{A}=1.03 \mathrm{GeV}$ for the axial mass describing the CCQE cross-section.

The implementation of the free nucleon CCQE cross-section in the NUANCE event generator follows the formalism described above, with two sophistications. First the value of the induced scalar form factor is not set to zero in NUANCE, and a value obtained by lattice QCD calculations is assumed instead [118]. Second, a 


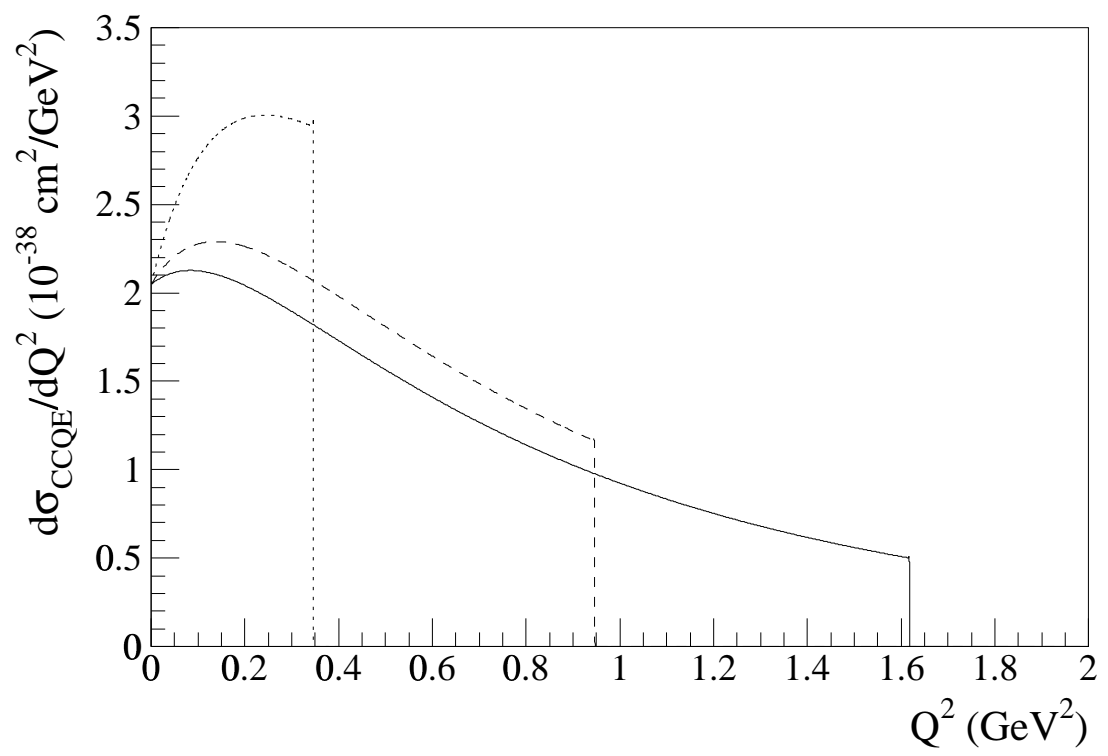

Figure 5.2: Free nucleon, differential charged-current, quasi-elastic cross-section per nucleon $d \sigma_{C C Q E} / d Q^{2}$ as a function of $Q^{2}$, based on Eq. 5.6. The three curves shown are for different neutrino energies $E_{\nu}$ : dotted for $E_{\nu}=0.4 \mathrm{GeV}$, dashed for $E_{\nu}=0.8 \mathrm{GeV}$, and solid for $E_{\nu}=1.2 \mathrm{GeV}$.

$Q^{2}$-dependence for the Sachs electric and magnetic form factors of the proton and nucleon that is more general than the simple dipole form is assumed instead [119]. Both effects have a negligible effect on the muon neutrino, free nucleon CCQE crosssection at MiniBooNE energies, and have been neglected in the discussion (but not in the results presented) for the sake of simplicity.

Figure 5.2 shows the $Q^{2}$ and $E_{\nu}$ dependence of the differential $\nu_{\mu}$ CCQE crosssection $d \sigma / d Q^{2}$ on a free target nucleon, based on the Llewellyn Smith CCQE formula 108. described above and adopted by NUANCE. In the limit $d \sigma / d Q^{2} \rightarrow 0$, the CCQE cross-section is neutrino-energy independent, while the dependence at $Q^{2} \neq 0$ is more complicated. Figure 5.2 also shows the kinematic limit $Q_{\max }^{2}$ allowed for a fixed neutrino energy $E_{\nu}$; neglecting the muon mass and for a stationary neutron, this is given by $Q_{\max }^{2}=4 E_{\nu}^{2} /\left(1+2 E_{\nu} / m_{N}\right)$.

In Figure 5.3, we show the comparison of the NUANCE computation of CCQE neutrino scattering on free nucleons with experimental data on $\nu_{\mu}$ CCQE scattering 


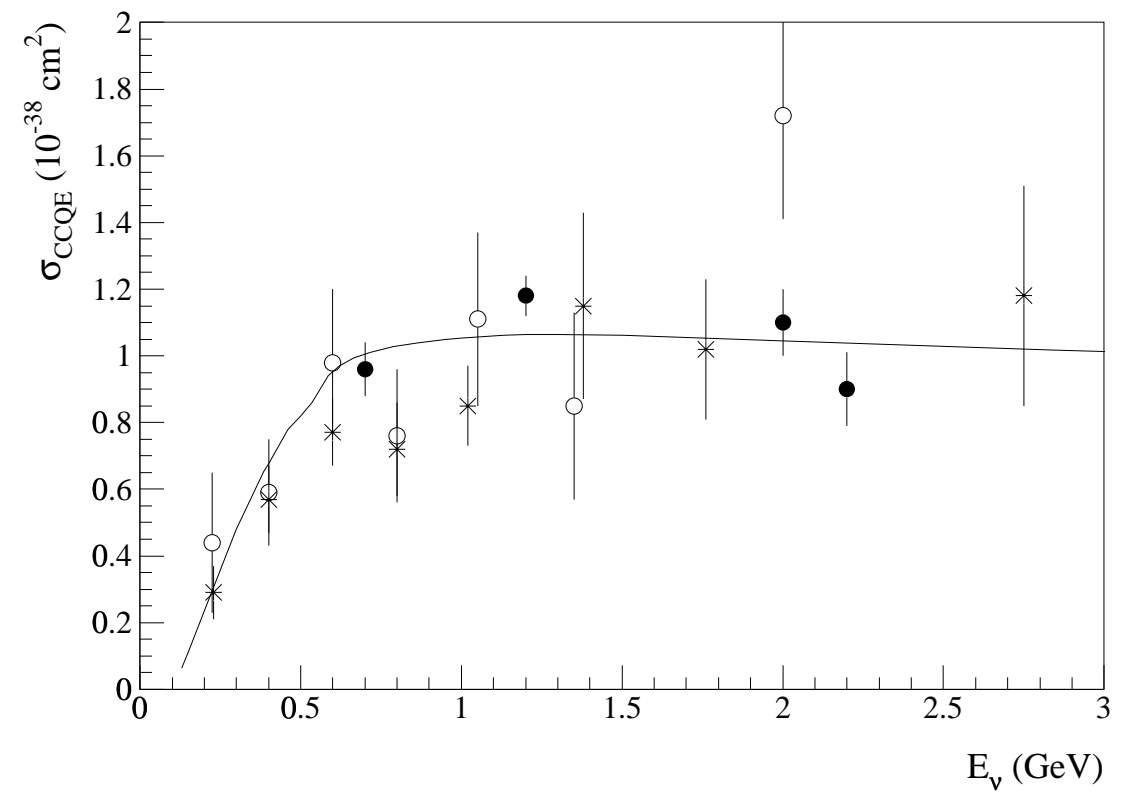

Figure 5.3: Total charged-current, quasi-elastic cross-section per nucleon $\sigma_{C C Q E}$ as a function of neutrino energy $E_{\nu}$, as measured by a number of neutrino scattering experiments using deuterium $\left(D_{2}\right)$ targets: * points from [120]; ○ points from [102]; • points from [121]. The solid line shows the NUANCE free nucleon prediction, with an axial mass $m_{A}$ of 1.03 GeV.

on deuterium $\left(\mathrm{D}_{2}\right)$. The comparison in Fig. 5.3 is restricted to deuterium target data since in this case the nuclear effects can be neglected.

\subsection{Nuclear Effects}

The target neutrons in MiniBooNE $\nu_{\mu} n \rightarrow \mu^{-} p$ interactions are bound in Carbon nuclei, and nuclear effects on CCQE (and other) interactions play an important role. Nuclear effects can be divided into three main categories: Fermi motion of the target nucleons, Pauli suppression of the phase space available to final state protons, and final state interactions (FSI) [108]. The Fermi motion of the neutron is particularly relevant in changing the interaction kinematics, the proton Pauli suppression causes a $Q^{2}$-dependent suppression of the CCQE cross-section, and FSI can change the 
composition and kinematics of the hadronic part of the final state ( $p$ for CCQE interactions).

The effect of Fermi motion and Pauli suppression on the CCQE cross-section is computed in NUANCE within the Smith and Moniz formalism [122, assuming a simple zero-temperature, relativistic Fermi gas model for the target nucleons and protons. In this model, the neutron and proton occupation numbers in the nucleus are momentum-independent and non-zero up to the Fermi momentum $p_{F}$, and zero for momenta $p>p_{F}$. This model for nuclear matter is fully specified by defining the Fermi momentum $p_{F}$ and nuclear binding energy $E_{B}$. In NUANCE, these values for Carbon are set to $p_{F}=220 \mathrm{MeV} / \mathrm{c}$ and $E_{B}=25 \mathrm{MeV}$. We now discuss the three main nuclear effects on CCQE interactions.

\subsubsection{Pauli Suppression}

We first discuss Pauli blocking. The CCQE cross-section suppression $S$ at a given momentum transfer $|\vec{q}| \equiv\left|\overrightarrow{p_{1}}-\overrightarrow{p_{3}}\right|$ due to Pauli blocking can be calculated analytically for a Fermi gas model; neglecting binding energy effects and for equal numbers of protons and neutrons (which is the case for Carbon), one gets a suppression $S=$ $1-D / N$, where $N$ is the number of neutrons in the nucleus, and $D$ is given by [123, 124]:

$$
D= \begin{cases}\frac{1}{2} A\left[1-\frac{3}{2} \frac{|\vec{q}|}{2 p_{F}}+\frac{1}{2}\left(\frac{|\vec{q}|}{2 p_{F}}\right)^{3}\right], & \text { for }|\vec{q}|<2 p_{F} \\ 0, & \text { for }|\vec{q}|>2 p_{F}\end{cases}
$$

where $A$ is the atomic weight ( $A=12$ for Carbon).

The suppression of the CCQE cross-section at a fixed momentum transfer $|\vec{q}|$ given by Eq. 5.17 and Carbon is shown in Fig. 5.4 ); as expected, the suppression is maximal at very low momentum transfers, and is absent if the neutron and proton Fermi spheres are completely disjoint $\left(|\vec{q}|>440 \mathrm{MeV} / \mathrm{c} \Rightarrow Q^{2}>0.18 \mathrm{GeV}^{2}\right)^{3}$. The

\footnotetext{
${ }^{3}$ The conversion from 3-momentum transfer $|\vec{q}|$ to $Q^{2}$ is done here for a stationary neutron, in which case: $Q^{2}=2 m_{N}^{2}\left[\sqrt{1+|\vec{q}|^{2} / m_{N}^{2}}-1\right]$.
} 

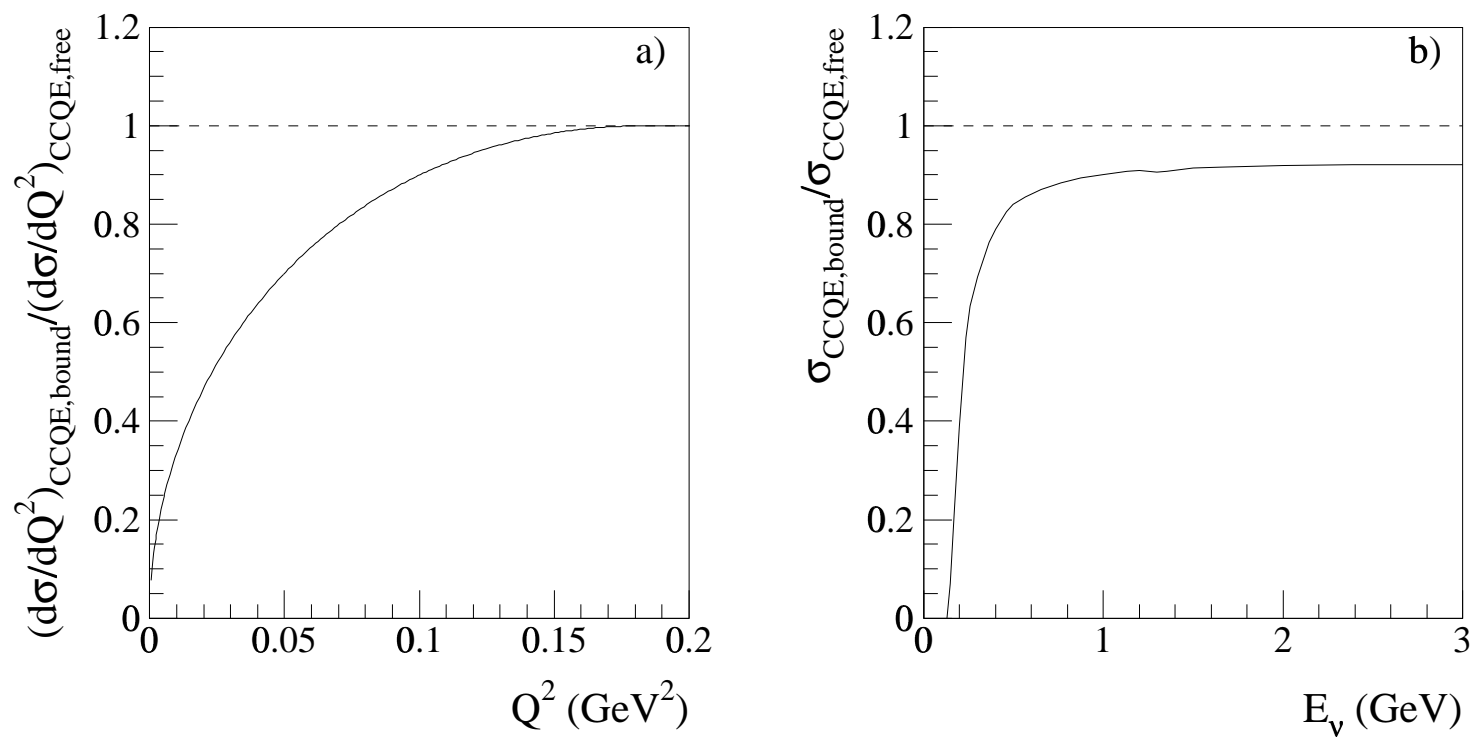

Figure 5.4: Effect of Pauli blocking on the CCQE cross-section. Figure 5.4a) shows the suppression factor $S$ at fixed $Q^{2}$, as given by Eq. 5.17; Fig. 5.4b) shows the suppression on the total cross-section as a function of neutrino energy $E_{\nu}$, as calculated by NUANCE.

Pauli suppression of the CCQE cross-section is also shown in Fig. 5.4b) as a function of neutrino energy $E_{\nu}$, as calculated by NUANCE. The suppression is particularly important at low neutrino energies; at $>1 \mathrm{GeV}$ neutrino energies, the bound nucleon CCQE cross-section for Carbon is about 92\% of the free nucleon CCQE cross-section.

Figure 5.5 compares the NUANCE predictions for the CCQE cross-section as a function of energy, both assuming free neutrons and for neutrons bound in ${ }^{12} \mathrm{C}$ nuclei, with all of the existing low-energy neutrino CCQE scattering data. Data taken with deuterium targets should be compared with the free nucleon NUANCE curve, while heavier target data should be more properly compared to the bound nucleon NUANCE curve. Overall, the NUANCE predictions agree well with the measurements, given the limited accuracy (10-20\%) of the measurements. 


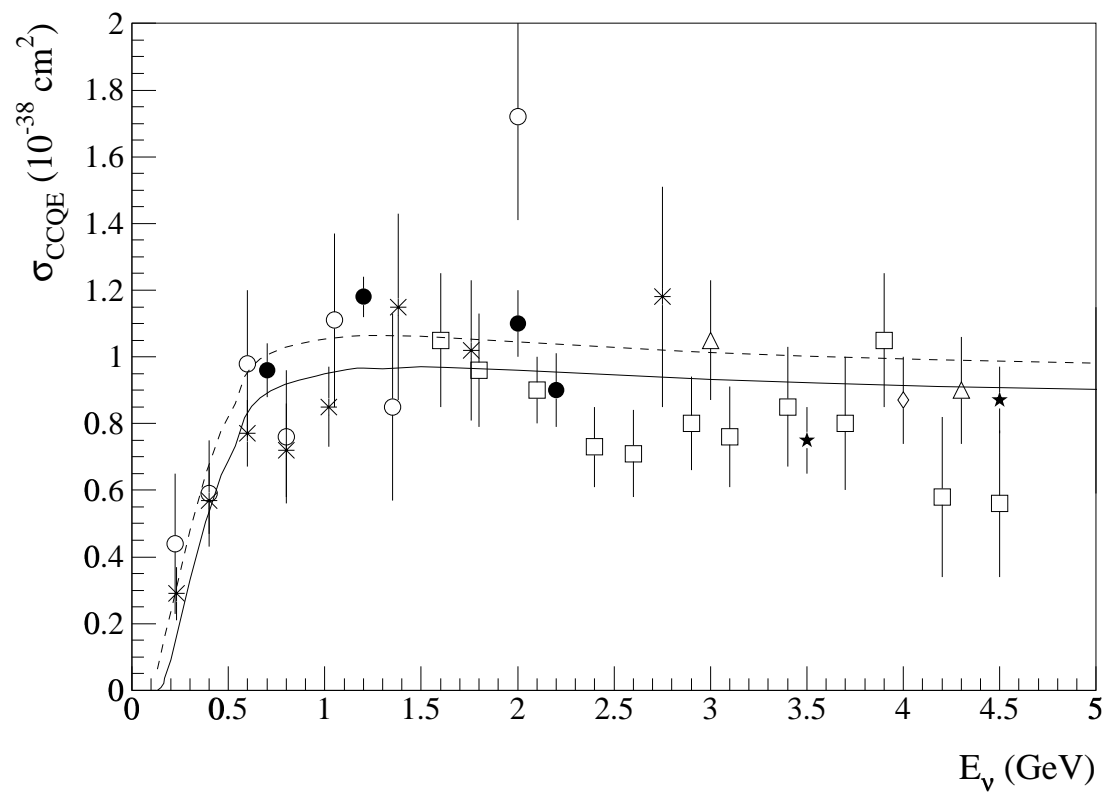

Figure 5.5: Total charged-current, quasi-elastic cross-section $\sigma_{C C Q E}$ per target nucleon as a function of neutrino energy $E_{\nu}$. The dashed line is the free-nucleon NUANCE calculation given also in Fig. 5.3, the solid line is the NUANCE calculation assuming neutrons bound in ${ }^{12} \mathrm{C}$ nuclei. Both computations assume $m_{A}=1.03 \mathrm{GeV}$ as the value for the axial mass. These predictions are compared with data from a variety of light and heavy target neutrino scattering data: deuterium (* points from [120]; O points from [102]; • points from [121]), propane-freon ( $\square$ points from [125]), heavy freon ( $\triangle$ points from [126]), aluminum ( $\diamond$ points [127]; * points from [128]).

\subsubsection{Fermi Momentum}

We now turn to the Fermi momentum of the bound target neutron. The Fermi motion does not cause a significant variation of the total cross-section as a function of the laboratory neutrino energy, but rather changes the kinematics of the CCQE interaction. To illustrate this point, we consider the quasi-elastic peak of the CCQE cross-section in the $\left(\nu, Q^{2}\right)$ plane, where we define $\nu$ as the energy transfer between the lepton (or hadron) part of the interaction: $\nu \equiv E_{\nu}-E_{\mu}$. In the CCQE process a neutrino scatters off the entire nucleus, and therefore the CCQE cross-section is completely determined by the kinematics: $d^{2} \sigma / d Q^{2} d \nu \propto \delta\left(Q^{2}-2 m_{N} \nu\right)$, if one neglects 
Fermi momentum and binding energy. The simple CCQE kinematic relationship $Q^{2}=2 m_{N} \nu$ gets modified in two ways in the presence of Fermi momentum $p_{F}$ and binding energy $E_{B}$. First, the reaction cannot occur unless $\nu=E_{\nu}-E_{\mu}=$ $E_{p}-\left(E_{n}-E_{B}\right)>E_{B}$, since the Fermi gas model and Pauli suppression impose the $\left|\vec{p}_{n}\right| \leq p_{F}$ and $\left|\vec{p}_{p}\right|>p_{F}$ constraints. Second, for a fixed $\nu$ value, a non-zero range of $Q^{2}$ values are now kinematically allowed: $Q_{\min }^{2}<Q^{2}<Q_{\max }^{2}$, where:

$$
\begin{array}{r}
Q_{\text {max } \min }^{2}=-2 m_{N}^{2}+2 E_{n} E_{B}-E_{B}^{2}+2\left(\nu+E_{n}-E_{B}\right) E_{n}- \\
2\left(\nu+E_{n}-E_{B}\right) E_{B} \pm 2 p_{F} \sqrt{\left(\nu+E_{n}-E_{B}\right)^{2}-m_{N}^{2}}
\end{array}
$$

where $E_{n}=\sqrt{p_{F}^{2}+m_{N}^{2}}$, and the minimum/maximum allowed $Q^{2}$ values correspond to the case where the outgoing proton is directed along or opposite to the incoming neutron direction, respectively, and the neutron momentum equals the Fermi momentum. The spread in the kinematic variables introduced by the Fermi motion is considerable, as can be seen from the 2D histogram of CCQE events in the $\left(\nu, Q^{2}\right)$ plane predicted by NUANCE, shown in Fig. 5.6. Figure 5.6 also shows the the analytic constraints on the CCQE kinematics derived above in the presence of Fermi momentum (for $p_{F}=220 \mathrm{MeV} / \mathrm{c}$ ) and binding energy (for $E_{B}=25 \mathrm{MeV}$ ), and the results agree well with NUANCE.

\subsubsection{Final State Interactions}

Finally, we discuss final state interactions. Hadrons produced in neutrino interactions are subject to final state interactions in their way out of the nucleus. NUANCE simulates pion-nucleon, kaon-nucleon, and nucleon-nucleon interactions based on standard PDG cross-sections [60], including charge exchange, pion absorption inelastic scattering, and elastic scattering. As a result, FSI change both the composition and the kinematics of the final state produced in the neutrino interaction. In particular, final state interactions play a significant role in determining the background to CCQE events in MiniBooNE. As discussed in Chapter 7, the main background to CCQE 

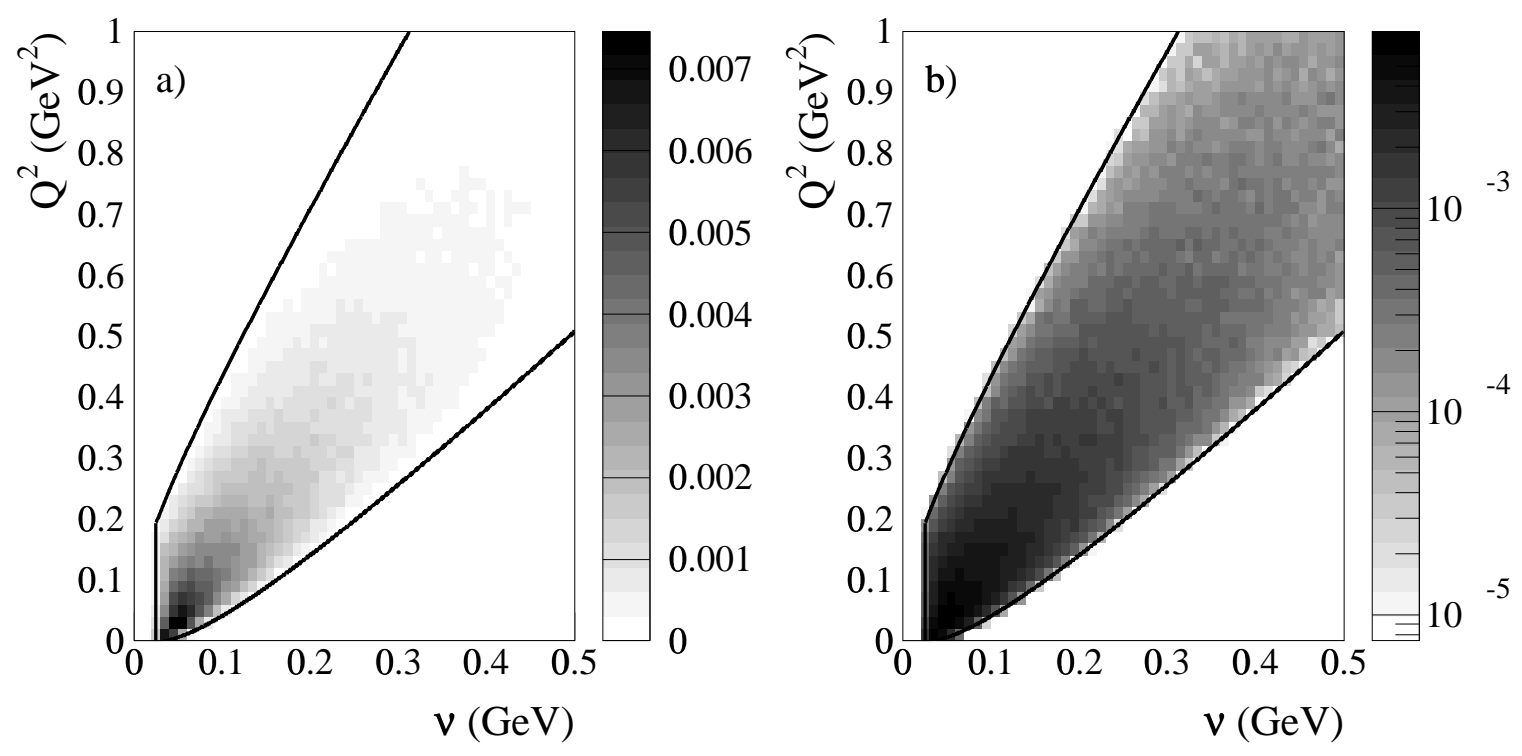

Figure 5.6: Effect of Fermi motion and binding energy on CCQE kinematic variables. The $2 D$ histogram shows $Q^{2} \equiv-\left(p_{\nu}-p_{\mu}\right)^{2}$ versus $\nu \equiv E_{\nu}-E_{\mu}$ for CCQE events generated with NUANCE, without any detector reconstruction effects (i.e., a perfect detector). The area enclosed by the solid line is the one derived in the text, assuming $E_{B}=25 \mathrm{MeV}$ and $p_{F}=220 \mathrm{MeV} / \mathrm{c}$. The panel a) uses a linear scale to display the fractional event yields per bin, while panel b) uses a logarithmic scale.

events is due to charged-current, resonant single pion production. Following this reaction, the produced pion (typically with a kinetic energy of a few hundred MeV) can be absorbed within the nucleus and cannot be observed. As an example of validation of the NUANCE treatment of final state interactions, Figure 5.7 shows the NUANCE prediction for the $\pi^{+}$absorption cross-section as a function of the pion kinetic energy in the range of relevance for MiniBooNE neutrino interactions, and how well the prediction compares with data [129]. The available data constrain the pion absorption cross-section in Carbon simulated in NUANCE at the 20-30\% level.

In order to quantify the effect of final state interactions in limiting the ability to identify "genuine" CCQE interactions even in a perfect neutrino detector, we can categorize the simulated interactions based on the types of particles present in the 


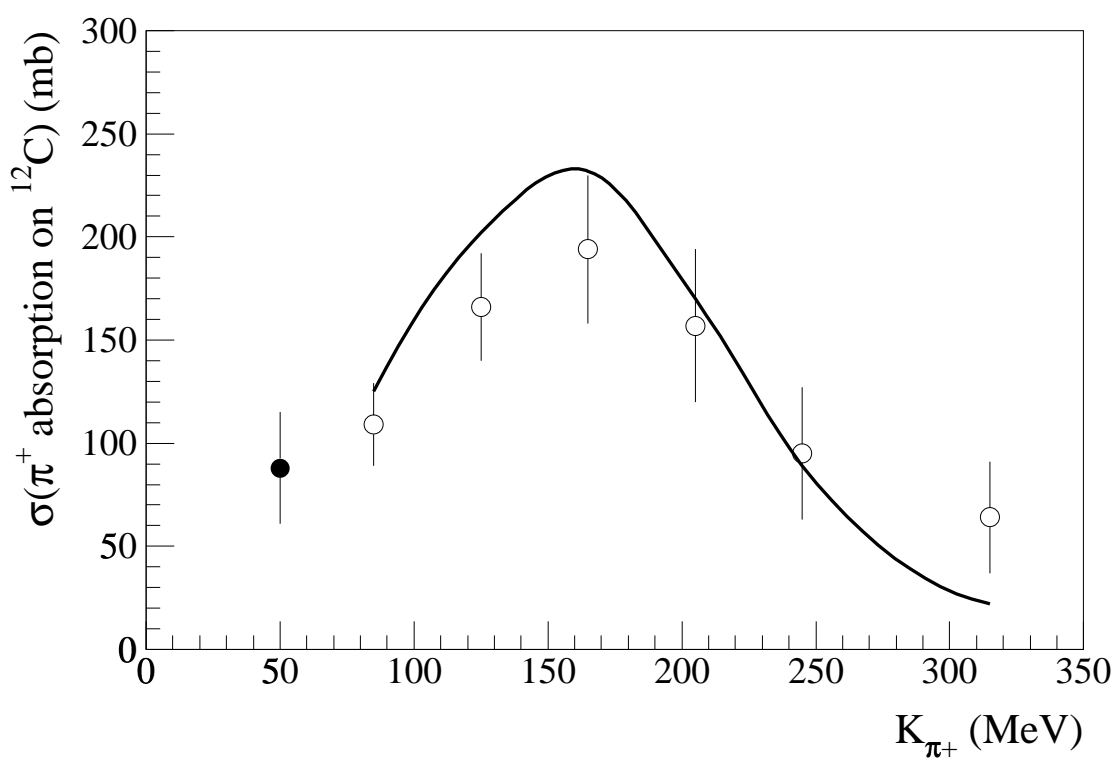

Figure 5.7: Example of validation of NUANCE treatment of final state interactions: $\pi^{+}$ absorption cross-section in Carbon as a function of $\pi^{+}$kinetic energy $K_{\pi^{+}}$. The plot shows the NUANCE predictions overlaid with experimental data: o points from [129], - points from [130].

final (observable) state, as opposed to classify them according to the type of underlying physics mechanism responsible for the interaction. We define interactions as "charged-current, quasi-elastic like" (CCQElike) if only muons, nucleons and soft photons are present in the final state. The fraction of CCQElike events predicted by NUANCE for the MiniBooNE flux is then:

$$
\eta_{\mathrm{CCQE} \text { like }}=P(\mathrm{CCQElike} \mid \mathrm{CCQE}) \cdot \eta_{C C Q E}+P(\mathrm{CCQElike} \mid \overline{\mathrm{CCQE}}) \cdot \eta_{\overline{\mathrm{CCQE}}}
$$

where $P(\mathrm{CCQElike} \mid \mathrm{CCQE})(P(\mathrm{CCQElike} \mid \overline{\mathrm{CCQE}}))$ are the probabilities for a CCQE (nonCCQE) interaction to have a final state consistent with the final state expected for a CCQE interaction in the absence of final state effects (CCQElike final state), and $\eta_{\mathrm{CCQE}}$ and $\eta_{\mathrm{CCQE}}$ are the CCQE and non-CCQE fractions of neutrino interactions, respectively.

Table 5.2 shows the relevant numbers appearing in Eq. 5.19. According to NUANCE predictions, categorizing neutrino interactions based on their observable final 


\begin{tabular}{|c|c|c|c|}
\hline$\eta_{\mathrm{CCQE}}(\%)$ & $P(\mathrm{CCQElike} \mid \mathrm{CCQE})(\%)$ & $P(\mathrm{CCQElike} \mid \overline{\mathrm{CCQE}})(\%)$ & $\eta_{\text {CCQElike }}(\%)$ \\
\hline 39.9 & 97.7 & 9.4 & 44.6 \\
\hline
\end{tabular}

Table 5.2: Charged-current quasi-elastic events and final state interactions. The Table gives the fraction $\eta_{C C Q E}$ of CCQE events predicted by NUANCE assuming the MiniBooNE flux, and also the fraction of expected MiniBooNE events with a final state consistent with the

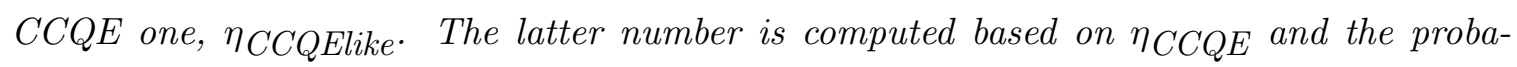
bilities for an event to "look" quasi-elastic given its CCQE or non-CCQE true identity: $P(C C Q E l i k e \mid C C Q E)$ and $P(C C Q E l i k e \mid \overline{C C Q E})$.

state increase the CCQElike fraction to $44.6 \%$, compared to a true CCQE fraction of $39.9 \%$. Figure 5.8 shows how the CCQElike and CCQE cross-sections compare as a function of neutrino energy, over the $0<E_{\nu}(\mathrm{GeV})<2$ neutrino energy range. Since resonant processes become more and more important with increasing energy compared to the quasi-elastic one, and since pion absorption is one of the main nuclear effects, it is not surprising that the effective CCQE cross-section increases as the neutrino energy increases. On the other hand, nuclear effects that would tend to produce a depletion in the effective CCQE cross-section, such as the pion production in the rescattering of a final state proton from a CCQE interaction with the Carbon nucleus, are simulated as well in NUANCE, but are less important than pion absorption. 


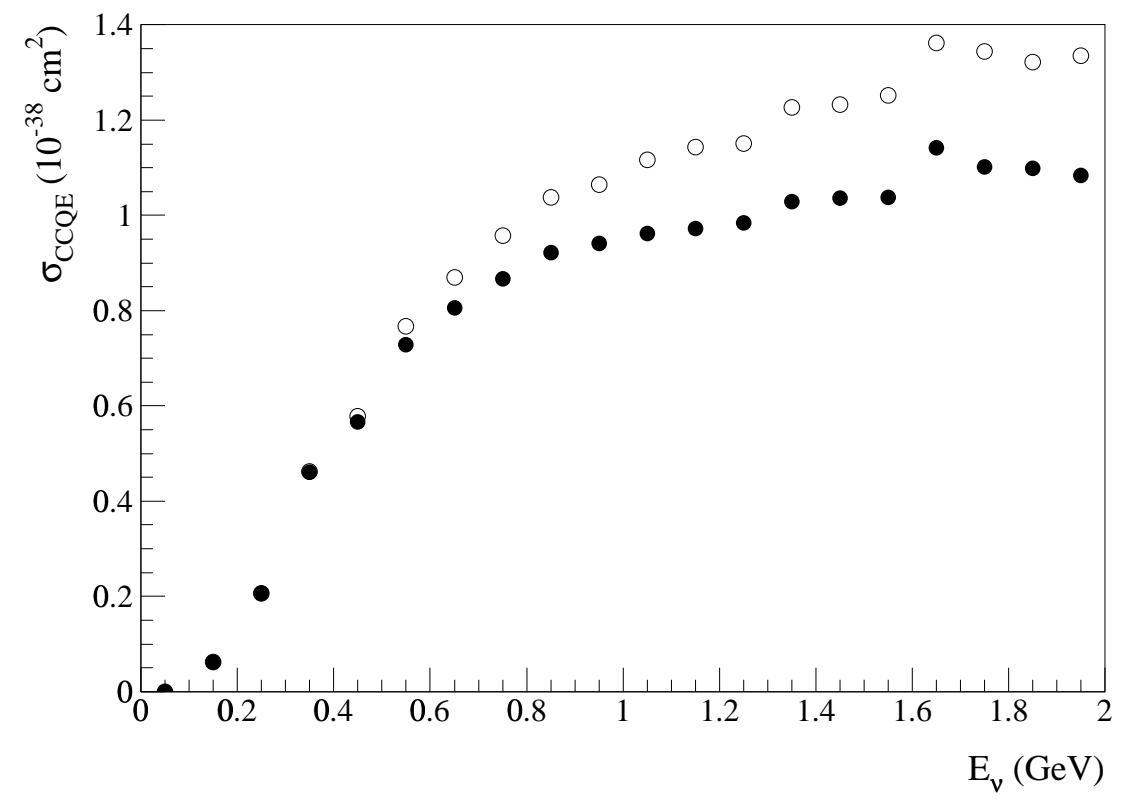

Figure 5.8: Comparison of the CCQE (solid circles) and "CCQElike" (empty circles) crosssections per nucleon as a function of neutrino energy $E_{\nu}$, as predicted by NUANCE. 


\section{Chapter 6}

\section{Overview of the MiniBooNE Detector}

\subsection{Overview of the MiniBooNE Detector Hard- ware}

The MiniBooNE neutrino detector consists of a carbon steel spherical tank of $6.1 \mathrm{~m}$ radius and filled with approximately 800 tons of undoped mineral oil. The center of the detector is located at a distance of $541 \mathrm{~m}$ from the neutrino production target, below a dirt overburden of about $3 \mathrm{~m}$. Schematics of the MiniBooNE site plan and of the MiniBooNE detector enclosure are shown in Figs. 3.1 and 6.1, respectively.

Neutrino interactions in the oil are observed by detecting the Cherenkov and scintillation photons produced by neutrino-induced charged tracks traveling in the detector medium. An array of 1,280 photomultiplier tubes (PMTs), located at a radius of 5.75 $\mathrm{m}$ and oriented toward the center of the tank, is used to record the number and arrival time of the photons produced in the fiducial volume of the detector. The PMTs provide a uniform, $10 \%$ coverage of the whole detector spherical inner surface. The spherical detector shell at $5.75 \mathrm{~m}<r<6.1 \mathrm{~m}$ is optically isolated from the main 


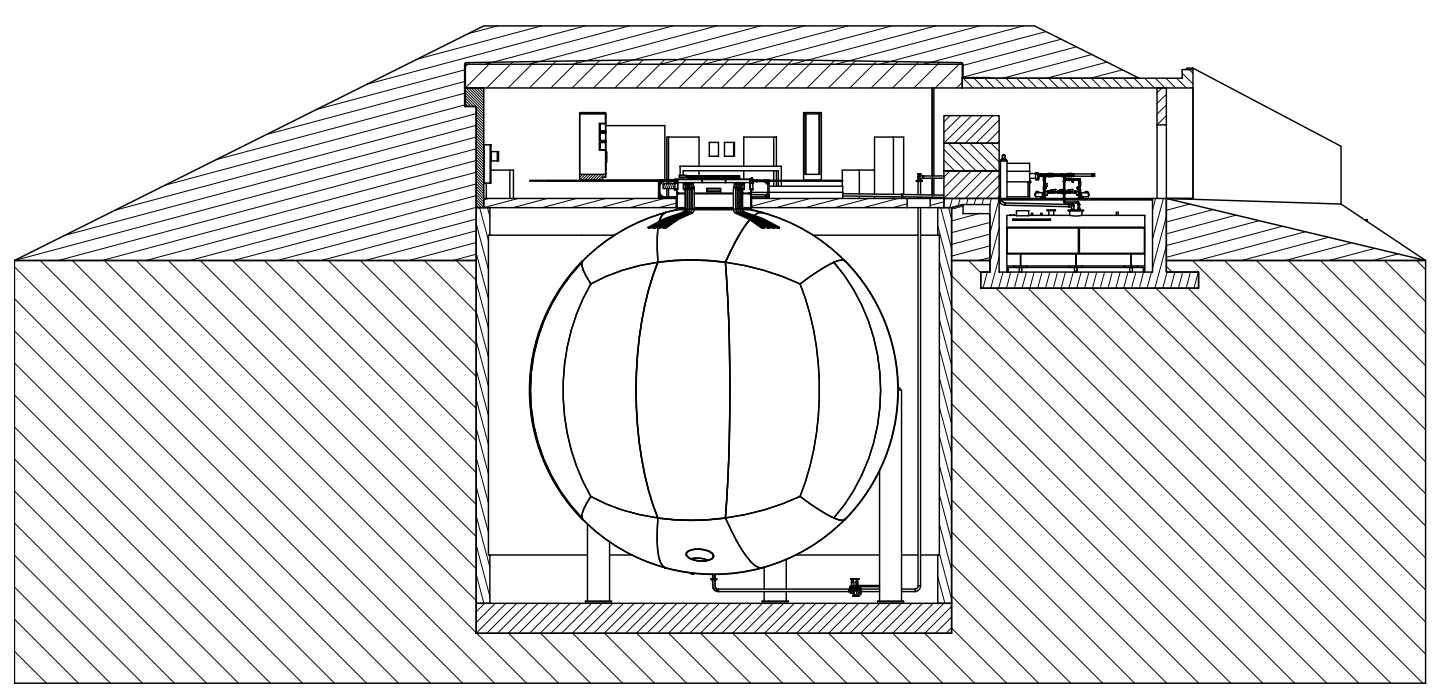

Figure 6.1: Schematic of the MiniBooNE detector enclosure, showing the vault containing the spherical detector, the electronics/utilities area located above the detector, and the dirt overburden.

detector region, and serves as a veto region to reject cosmic-ray induced activity in the tank. Photons produced in the veto region are read out by 240 PMTs of the same type employed in the main detector region. The photoelectron charge and time of the PMT signals are continuously digitized and recorded for every proton beam spill. A laser system, a cosmic ray muon hodoscope, and seven scintillation cubes located inside the detector, are used to calibrate various aspects of the MiniBooNE detector response. Hardware details related the MiniBooNE mineral oil, PMT readout, data acquisition system, trigger, and calibration devices follow. More details can be found in [131].

\subsubsection{The Nuclear Target: MiniBooNE Mineral Oil}

Neutrino interactions in MiniBooNE proceed primarily via interactions with mineral oil, since the 810 tons of oil filling the detector comprise about $95 \%$ of the total detector tonnage. The remaining 5\% detector tonnage consists of the $1 \mathrm{~cm}$ thick steel spherical tank, the optical barrier supporting the PMTs, the PMTs themselves, and cables. The interactions from nuclear targets other than mineral oil in the detec- 
tor, and from the dirt surrounding the detector, are negligible once a fiducial volume requirement is applied to study muon neutrino, charged-current quasi-elastic interactions (see Chapter 7).

The mineral oil used in MiniBooNE is Marcol 7, manufactured by Exxon / Mobil. This oil was chosen for its long light attenuation length, high index of refraction and small dispersion over the wavelength range $280-640 \mathrm{~nm}$, its low viscosity, its low reactivity with materials in the detector, and its scintillation light production. The Marcol oil density is $0.855 \mathrm{~g} / \mathrm{cm}^{3}$. Details on the mineral oil optical properties of relevance to the MiniBooNE detector response are given in Sections 6.2.1 and 6.2.2.

The oil is kept at ambient temperature. An overflow tank with a capacity of $1 \%$ of the main tank volume is used for oil containment following thermal expansion. The oil can be recirculated and cooled at a rate of about 100 liters per minute, if necessary. Moreover, to maintain the detector optimal optical characteristics over time, a nitrogen gas layer above the oil is kept at all times to avoid exposure to air, and therefore minimize the oxygen impurities in the oil. If necessary, nitrogen gas can also be continuously bubbled into the detector.

\subsubsection{Optical Readout: MiniBooNE Photomultiplier Tubes}

The light produced by neutrino interactions is detected by 1,520 main and veto photomultiplier tubes. All main tubes are mounted on the inner side of a spherical support structure built within the detector, at a radius of $5.75 \mathrm{~m}$, while veto tubes are mounted on the carbon steel tank walls, at a radius of $6.1 \mathrm{~m}$. Figure 6.2 shows a picture of the actual installation of main and veto PMTs in the detector, prior to oil filling.

Most of the PMTs installed in MiniBooNE, 1196, are Hamamatsu R1408 PMTs recycled from the LSND experiment, and 324 are Hamamatsu R5912 PMTs that were newly purchased for the experiment. Both PMT models have a photocathode 


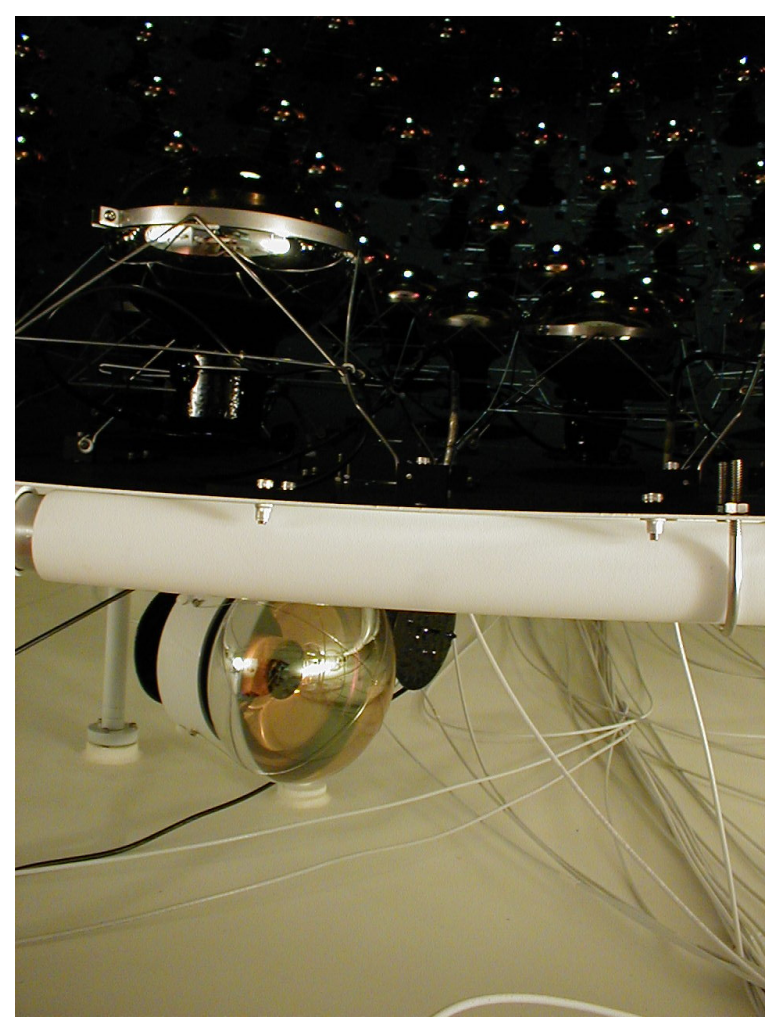

Figure 6.2: Picture of the MiniBooNE detector optical barrier and its PMT installation, before the detector was filled with mineral oil. The inner and outer surfaces are painted in black and white, respectively, to minimize and maximize light reflection.

diameter of $20 \mathrm{~cm}$. All R5912 PMTs, and the LSND R1408 PMTs offering the best performances [132], are mounted in the main detection region, and PMTs characterized by low dark rates were chosen for the veto region. Figure 6.3 shows a schematic of a MiniBooNE PMT with its mounting structure for one of the 1,280 main PMTs. The R1408 (R5912) tubes have a 9 (10) stage dynode chain amplification. The PMTs are operated at an average high voltage of about 1,800 Volts. The operating voltage is set separately for each tube, to ensure a uniform gain throughout the tank of $1.6 \cdot 10^{7}$, as dictated by MiniBooNE electronics. Typical dark noise rates of $3 \mathrm{kHz}$ for the MiniBooNE PMTs have been measured at ambient temperature and default operating voltages, low enough to satisfy the experiment's needs. Aspects of the PMT response that are of relevance to the event reconstruction are given in Section 6.2.3. 


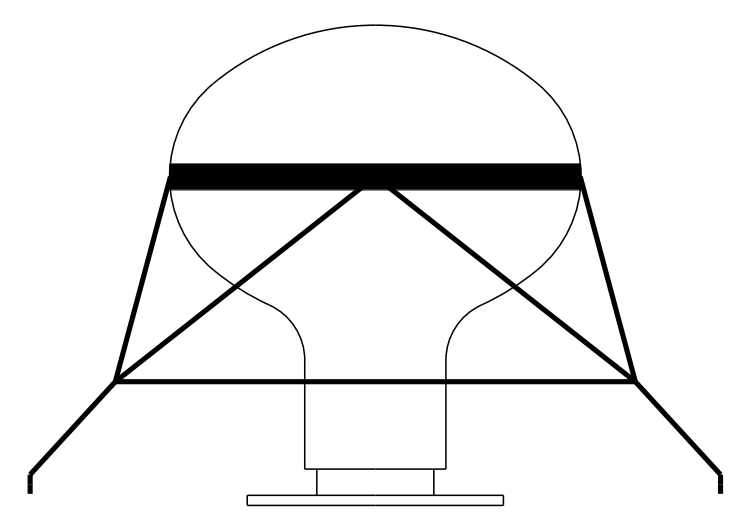

Figure 6.3: Schematic of a MiniBooNE PMT, together with its support frame used for installation in the MiniBooNE main detection region. To give a sense of scale, the diameter of the photocathode is about $20 \mathrm{~cm}$.

\subsubsection{Data Acquisition System and Trigger}

The PMT signals are routed to the electronics area located above the detector, where further amplification and digitization occurs. As for the PMTs, the electronics employed in MiniBooNE is also based on existing LSND hardware, modified to fit the different needs of the experiment.

Because of the high number of channels and the DAQ rate requirements, a full digitization of the PMT signal waveforms is not performed; rather, only the overall charge and time associated with each PMT hit is recorded. A schematic of the charge and time signal formation is shown in Fig. 6.4. The preamplified PMT signals $V_{\text {pmt }}$ are continuously integrated via a capacitive circuit contained in the MiniBooNE "QT boards", with an exponential decay time of about 1,200 ns, generating a second signal $V_{q}$. The QT boards (as in "charge/time boards") serve eight PMT channels each, and are arranged into twelve QT crates each hosting 16 QT boards, for a total of $12 \times 16 \times 8=1,536$ channels, enough to serve all the 1,520 MiniBooNE PMTs. The charge signal $V_{q}$ is continuously digitized by the DAQ, at $100 \mathrm{~ns}$ time intervals. If 


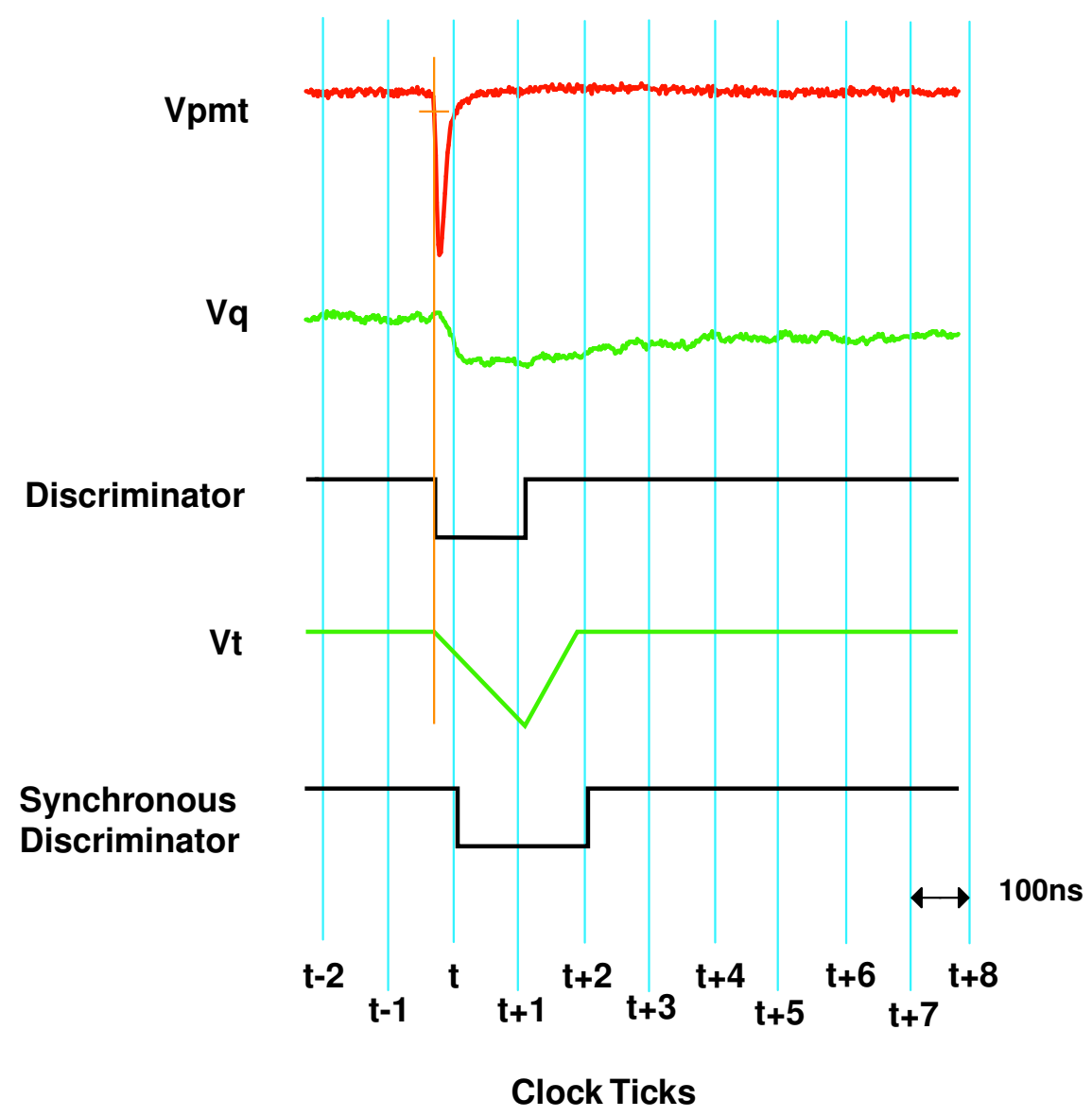

Figure 6.4: Schematic of the PMT charge and time digitization in MiniBooNE.

the PMT signal $V_{\text {pmt }}$ exceeds a voltage corresponding to about 0.25 photoelectrons, a PMT discriminator signal is activated, and a linear time ramp $V_{t}$ is started, as shown in Fig. 6.4. The time signal $V_{t}$ is also continuously digitized every $100 \mathrm{~ns}$. The purpose of the voltage time ramp is to obtain a precise determination of the time at which the PMT signal crosses threshold, since much better than 100 ns time accuracy is necessary for MiniBooNE event reconstruction. The time ramp is reset to baseline when two 100 ns time intervals are elapsed since the time at which the PMT signal crosses threshold.

The trigger logic is designed to decide whether or not to capture a fixed DAQ time window of PMT charge and time data for all PMTs. The trigger queries the status of several trigger conditions to make this decision. Some trigger conditions use 
detector data only, for example requiring a minimum number of PMT signals above threshold in the main detector region. However, the most important trigger condition is enabled whenever protons are delivered from the Fermilab Booster accelerator to the MiniBooNE target hall, regardless of the detector status. All neutrino-induced events described in the following are selected based on this trigger condition. The time window captured by this beam trigger is $19.2 \mu \mathrm{s}$ long, timed such that the $1.6 \mu \mathrm{s}$ long beam spill occurs at $4.6<\Delta t(\mu \mathrm{s})<6.2$, where $\Delta t$ is the time with respect to the beginning of the time window. The duration of the time window is chosen to be able to detect the electrons from muons decaying at rest in the detector over about 6 muon lifetimes. Other important external trigger conditions are set up for detector calibration (discussed in Sections 6.1.4 and 6.3.2), and for detecting random windows in time to study the detector activity in an unbiased way.

Whenever a trigger condition is met, an event with the following information is recorded for each PMT channel: the PMT channel number, the 100 ns time interval that precedes the discriminator firing (the one corresponding to the $(t-1)$ time in Fig. 6.4), four digitized $V_{q}$ values, and the corresponding four digitized $V_{t}$ values, the one that precedes the discriminator firing, and the three subsequent ones. In addition, in the rare occurrence that charge exceeds the ADC dynamic range of about 20 PEs, a variation on the DAQ scheme described above is adopted to fully retrieve the PMT charge information.

This digitization scheme allows the recovery of the intrinsic charge and time resolution of the PMTs, without introducing any additional smearing associated with the data acquisition itself. The MiniBooNE PMT charge and time response calibrations, which are of direct relevance to the reconstruction of neutrino interactions in the detector, are given in Section 6.2.3. 


\subsubsection{Calibration Devices}

The detector is equipped with two systems for its hit-level and reconstruction-level calibration, discussed in Sections 6.2.3 and 6.3.2, respectively. These two calibration systems complement the reconstruction-level calibration samples that can be extracted using MiniBooNE detector information only.

\section{Laser/flask system}

A diode laser located in the detector electronics area can deliver sub-ns light pulses into the detector. The laser light wavelength can be set to either $397 \mathrm{~nm}$, or $438 \mathrm{~nm}$; the laser light intensity and repetition rate are adjustable, and of the order of $\mathcal{O}(\mu \mathrm{W})$ and $\mathcal{O}(\mathrm{Hz})$, respectively. The light is transmitted from the laser to the detector via five optical fibers. Four optical fibers are terminated with flasks filled with dispersive material, yielding a nearly isotropic laser light emission from the flasks. The four flasks are located at various positions within the detector, with one flask positioned at the detector center. The fifth optical fiber is not coupled to a dispersive flask, and yields a collimated $\left(\sim 10^{\circ}\right.$ opening angle) beam of laser light in the mineral oil. A special calibration trigger enables to record the detector activity following laser light calibration events, which occur asynchronously with respect to the proton beam delivery to the MiniBooNE target hall 1 . As described in Section 6.2.3, laser events allow to calibrate the individual PMT responses.

\section{Cosmic Ray Muon Tracker and Scintillation Cubes}

Cosmic ray muons and their decay electrons provide extremely useful event samples for calibrating the detector as a whole, at the reconstruction-level (see Section 6.3.2). The cosmic ray muon hodoscope located above the detector and the scintillation cubes located inside the detector allow to record the detector activity due to muons

\footnotetext{
${ }^{1}$ Laser events are vetoed in the case of overlap with the beam spill.
} 
with known direction, and with known pathlength and energy deposition inside the detector.

The cosmic ray muon hodoscope consists of four scintillator planes, spatially separated into two layers of two planes each. The muon tracker trigger is enabled by a 4 -fold scintillator plane time coincidence. In a coordinate system where $\vec{y}$ points upwards and $\vec{z}$ along the neutrino beam direction, the two layers are separated by $\Delta y \equiv y_{\text {top }}-y_{\text {bottom }} \simeq 100 \mathrm{~cm}$. The two planes of scintillator strips in each layer are oriented to provide one $(x, y)$ and one $(y, z)$ 2-dim coordinate, therefore providing a full 3-dimensional location. From the top and bottom layer 3-dimensional coordinates, the muon track direction and entry point inside the detector can be extracted. The muon tracker dimensions and location with respect to the detector allow to calibrate the detector track direction reconstruction algorithms over the the range $-1<$ $\cos \theta_{y}<\cos \theta_{y, \max },-\sqrt{1-\cos \theta_{y, \max }^{2}}<\cos \theta_{x}, \cos \theta_{z}<\sqrt{1-\cos \theta_{y, \max }^{2}}$, where $\cos \theta_{x}, \cos \theta_{y}, \cos \theta_{z}$ are the direction cosines of the muon track direction $\left(\cos \theta_{y}=-1\right.$ for downward-going muons), and $\cos \theta_{y, \max } \simeq-0.65$. Moreover, the muon tracker scintillator strip segmentation and location yields an angular resolution intrinsic to the tracker of about $30 \mathrm{mrad}$, which is smaller than the typical $100 \mathrm{mrad}$ angular spread expected for multiple Coulomb scattering of muons in the detector [133].

Seven optically isolated cubes, made of scintillator material and of a few $\mathrm{cm}$ on a side, are deployed inside the MiniBooNE detector. The scintillation light produced by a charged particle moving inside a cube is collected by an optical fiber, and directed to a 1 inch PMT located outside the detector. The cube trigger is enabled whenever a time-delayed coincidence between two light pulses from the same cube are detected by the PMT readout, consistent with an event having a muon reaching and stopping inside a cube (first light pulse), followed by a second scintillation light pulse produced by the muon decay electron along its path inside the cube. Therefore, cube events allow the determination of the stopping position of a muon inside the MiniBooNE detector to a few cm accuracy, including the cube survey position accuracy. The cubes 
are located at various positions inside the detector, so that the distances between the cubes and the detector surface range from 15 to about $400 \mathrm{~cm}$. Given the energy loss of muons in oil, the cube positions allow to study muons that deposit between 20 and $800 \mathrm{MeV}$ of energy inside the tank [134].

The coincidence between a muon tracker trigger signal and a scintillation cube trigger select muons with known direction and entry point position (from the tracker), and known stopping point (from the cube); from the entry and stopping points, the muon pathlength and the energy deposition inside the detector are determined. On an event-by-event basis, the muon energy resolution measured by the tracker/cube calibration system is dominated by the few \% fluctuations in energy loss (range straggling).

\subsection{The MiniBooNE Detector Response}

\subsubsection{Light Production}

Optical photons ${ }^{2}$ are produced in the mineral oil by charged tracks via the mechanisms of Cherenkov and scintillation light production.

\section{Cherenkov Light}

Cherenkov light production in a dispersive medium with index of refraction $n$, by a relativistic particle of charge $z e$ and moving at a velocity $\beta>1 / n$, is given by [60]:

$$
\frac{d^{2} N}{d x d \lambda}=\frac{2 \pi \alpha z^{2}}{\lambda^{2}} \sin ^{2} \theta_{C}
$$

where $d^{2} N / d x d \lambda$ is the number of Cherenkov photons emitted per particle's unit path length $x$ and per unit photon wavelength $\lambda, \alpha=e^{2} /(\hbar c)$, and $\theta_{C}$ is the angle of

\footnotetext{
${ }^{2}$ We define here optical photons as photons in the $250<\lambda(\mathrm{nm})<650$ wavelength range.
} 
Cherenkov light emission with respect to the particle's direction, given by:

$$
\cos \theta_{C}=\frac{1}{\beta n(\lambda)}
$$

The refractive index $n$ of the MiniBooNE mineral oil is well described by the Cauchy parametrization:

$$
n(\lambda)=n_{D}+B\left(\frac{1}{\lambda^{2}}-\frac{1}{\lambda_{D}^{2}}\right)
$$

where $\lambda$ is the photon wavelength in $\mathrm{nm}, \lambda_{D}=589.3$, and the parameters at $20 \mathrm{degC}$ have been measured to be $n_{D}=1.4684 \pm 0.0002$ and $B=(4240 \pm 157) \mathrm{nm}^{2}$ [135, 136].

As the particle slows down (decreasing $\beta$ ), Cherenkov light is emitted at smaller angles $\theta_{C}$, and the number $N$ of Cherenkov photons produced decreases. Cherenkov light production is absent if $\beta<1 / n(\lambda)$. As shown in Fig. 6.7a), the MiniBooNE PMTs are sensitive to photons over the wavelength range $280<\lambda(\mathrm{nm})<640$; the Cherenkov flux yield per unit track length for muons, integrated over this wavelength range and as a function of the muon momentum $p_{\mu}$, is shown in Fig. 6.5. ${ }^{3}$.

A typical $300 \mathrm{MeV} / \mathrm{c}$ muon produces about 450 Cherenkov photons per $\mathrm{cm}$ in mineral oil, in the $280<\lambda(\mathrm{nm})<640$ photon wavelength range. For this muon momentum and in this photon wavelength range, the mean and RMS of the photon wavelength spectrum are $410 \mathrm{~nm}$ and $99 \mathrm{~nm}$, respectively; as discussed Sections 6.2.2 and 6.2.3, the Cherenkov emission spectrum matches well the photon wavelength range of the MiniBooNE detector response. Moreover, the mean and RMS of the cosine of the Cherenkov angle in the same photon wavelength range $280<\lambda(\mathrm{nm})<$ 640 and for the same muon momentum of $300 \mathrm{MeV} / \mathrm{c}$ are 0.714 and 0.006 , respectively; the angular distribution of Cherenkov emission is anisotropic. Finally, the Cherenkov light emission is prompt, and chromatic dispersion effects on the Cherenkov photon

\footnotetext{
${ }^{3}$ The MiniBooNE detector is somewhat sensitive to $\lambda<280 \mathrm{~nm}$ photons as well, since wavelength shifting processes, such as fluorescent emission discussed in Sec. 6.2.2, are present. For this reason, photon production in the $250<\lambda(\mathrm{nm})<650$ wavelength range is considered in the detector simulation.
} 

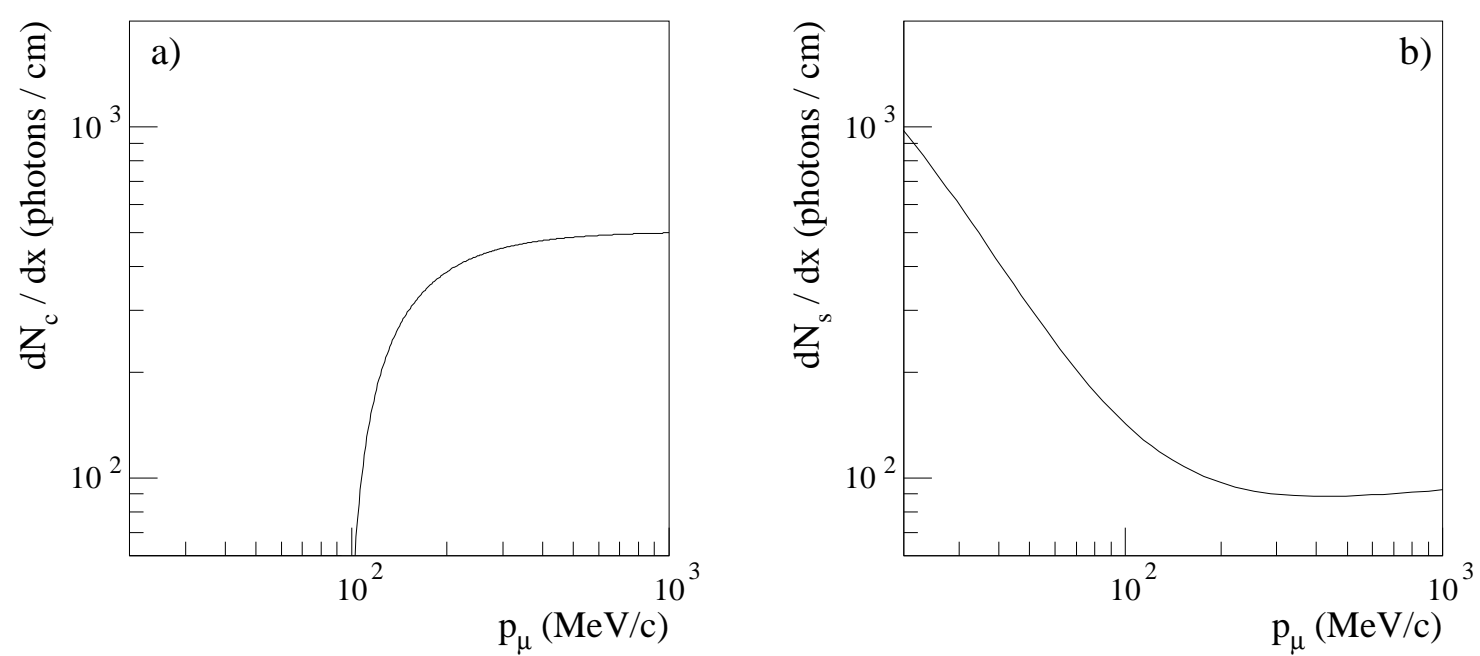

Figure 6.5: Number of optical photons produced per unit path length of muons in MiniBooNE mineral oil, as a function of muon momentum $p_{\mu}$. Figure 6.5a shows Cherenkov light production per unit path length, $d N_{c} / d x$, integrated over the photon wavelength range $280<\lambda(\mathrm{nm})<640$. Figure 6.5b shows scintillation light production per unit path length, $d N_{s} / d x$, integrated over the whole scintillation emission spectrum.

propagation (due to the variation of the group velocity with photon wavelength) are negligible.

\section{Scintillation and Fluorescence Light}

Charged tracks traversing mineral oil excite the target molecules because of energy deposition; the following de-excitation of the mineral oil organic molecules is accompanied by isotropic and delayed scintillation light emission. A related process is the excitation of target molecules by optical photons (as opposed to charged tracks); the following de-excitation of mineral oil molecules is accompanied by fluorescence light emission. In both cases, several fluorophores contribute to the light emission. The emission wavelength spectrum and the emission time spectrum of scintillation and fluorescence light depend on the details of the mineral oil chemical composition and molecular structure, and are determined experimentally. In particular, impurities present in the mineral oil, most notably the Vitamin E added to the oil as 
anti-oxydant, are believed to be responsible for scintillation and fluorescence light emission.

Scintillation and fluorescence light are only approximately proportional to the ionization loss of tracks in mineral oil, and recombination and quenching effects are believed to reduce the light yield for highly ionizing particles. The semi-empirical Birk's saturation law for energy deposition is typically used to convert the ionization energy loss per unit path length and unit material density, $d E / d x\left(\mathrm{MeV} /\left(\mathrm{g} \mathrm{cm}^{-2}\right)\right)$, into a corrected energy loss $d E^{\prime} / d x$ that is directly proportional to the light output 60]:

$$
d E^{\prime} / d x=\frac{d E / d x}{1+k_{B} d E / d x}
$$

where the Birk's constant $k_{B}$ is about $0.014 \mathrm{~g} \mathrm{~cm}^{-2} \mathrm{MeV}^{-1}$ for mineral oil.

The properties of scintillation light production are determined empirically, both from external measurements and from MiniBooNE detector data. The identification of the mineral oil fluorophores, the decay time constant of their exponential light emission, and their wavelength emission spectra, are determined from external measurement on time resolved fluorescence [137]. The light yield per energy deposited for each fluorophore is determined both from these external measurements, and from MiniBooNE detector calibration data using electrons from muon decays at rest. These properties are summarized in Tab. 6.1.

The total scintillation light yield per unit path length, for a muon traveling in mineral oil, is given in Fig. 6.5b as a function of muon momentum. A typical 300 $\mathrm{MeV} / \mathrm{c}$ muon produces about 90 scintillation photons per $\mathrm{cm}$ in mineral oil, integrated over the entire photon emission spectrum, $270<\lambda(\mathrm{nm})<460$. The typical photon wavelengths characterizing scintillation emission spectra for the various mineral oil fluorophores are shorter than the corresponding ones associated with Cherenkov light emission, and therefore scintillation photons do not match equally well the photon wavelength range of the MiniBooNE detector response; consequently, the typical $450: 90 \sim 5: 1$ ratio between Cherenkov and scintillation photons at production 


\begin{tabular}{|c|r|r|c|c|}
\hline Fluorophore & $d N_{s} / d E^{\prime}$ & $\tau(\mathrm{ns})$ & \multicolumn{2}{|c|}{ Emission Spectrum } \\
& $($ photons $/ \mathrm{MeV})$ & & $\operatorname{Mean}(\lambda)(\mathrm{nm})$ & $\operatorname{RMS}(\lambda)(\mathrm{nm})$ \\
\hline 1 & 24.84 & 14.0 & 303 & 13 \\
2 & 20.52 & 33.0 & 360 & 27 \\
3 & 5.94 & 1.0 & 331 & 18 \\
\hline Total & 51.30 & & & \\
\hline
\end{tabular}

Table 6.1: Properties of scintillation light emission spectra in MiniBooNE mineral oil. The properties of the three dominant fluorophores are listed. $d N_{s} / d E^{\prime}$ is the number of scintillation photons emitted per unit energy deposition, where the energy deposition is corrected for Birk's saturation law (see text for details); $\tau$ is the decay time constant of the exponential time distribution of the emitted light; Mean $(\lambda)$ and $R M S(\lambda)$ are the mean and RMS of the emission wavelength spectrum, respectively.

is translated in a larger ratio at detection. As already mentioned, scintillation light production is isotropic and characterized by delay times of the order of tens of ns (see Tab. 6.1).

\subsubsection{Light Transmission}

Optical photons typically have to traverse distances of the order of a few meters before reaching a PMT located on the optical barrier surface, and be detected. Light propagation over distance is affected mainly by three physics processes: photon absorption, scattering, and fluorescence. The term "photon attenuation" is used here to describe these three physics process cumulatively, and it is to be interpreted as the attenuation of photons measured along the direction specified by their initial direction at production. The individual absorption, scattering, and fluorescence rates, together with the overall attenuation rate, are shown in Fig. 6.6 as a function of the photon wavelength. The attenuation rate is defined as the number of photon interactions resulting in attenuation (as specified above) per unit path length. For typical photon 


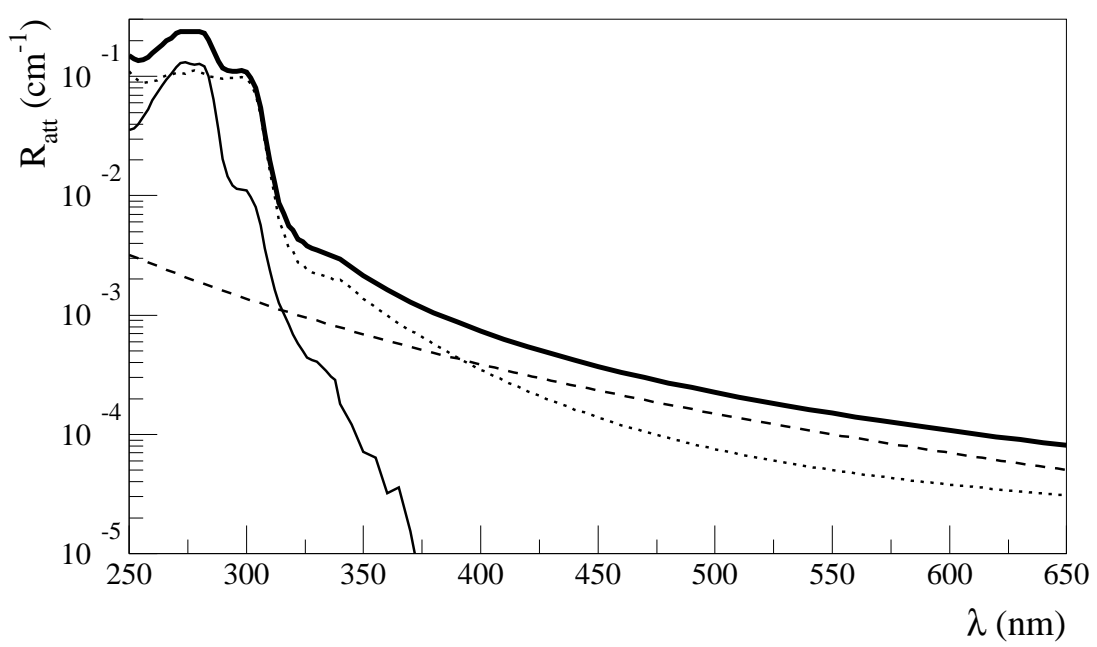

Figure 6.6: The attenuation rate $R_{\text {att }}$ in MiniBooNE mineral oil as a function of photon wavelength $\lambda$ is shown by the thick, solid curve. The individual components to the attenuation rate are also shown: fluorescence rate (thin, solid line), scattering rate (dashed line), absorption rate (dotted line).

wavelengths of $400 \mathrm{~nm}$, the attenuation rate is $R_{\text {att }} \simeq 7 \cdot 10^{-4} \mathrm{~cm}^{-1}$, corresponding to an attenuation length of $L_{\text {att }} \simeq 1 / R_{\text {att }} \simeq 14 \mathrm{~m}$, that is of the order of the MiniBooNE detector diameter [137]. For the same wavelength of $400 \mathrm{~nm}$, about half of the attenuation is interpreted as due to absorption, and half as due to to scattering.

\section{Fluorescence}

Fluorescence is the process in which an optical photon (created by either Cherenkov or scintillation light production processes) is absorbed by the mineral oil in the form of molecular excitation, and re-emitted at a longer wavelength, different direction, some time after absorption. Fluorescence measurements using samples of MiniBooNE mineral oil were carried out both at Johns Hopkins University and at Fermilab [137]. The emission spectra of fluorescent light have already been discussed in connection to scintillation light production. The measured relationship between absorption and emission spectra for the mineral oil fluorophores is typically characterized by Stokes' 
shifts of a few tens of nm, where the Stokes' shift refers to the wavelength difference between the peak of the absorption and emission spectra [60]. The measured contribution of fluorescence to attenuation becomes significant only for photon wavelengths below about $300 \mathrm{~nm}$; in this case, photons with longer wavelengths (and therefore subject to smaller attenuation rates) are emitted.

\section{Scattering}

Scattering is defined here as the process in which an optical photon interacts with target molecules, gets deflected and possibly changes its polarization state, but not its wavelength. Scattering measurements of photons in mineral oil were carried out at Princeton University [138]. The dominant contribution to scattering is measured to be due to isotropic Rayleigh scattering, although also anisotropic Rayleigh scattering and Raman scattering are observed, contributing to roughly $20 \%$ and $7 \%$ of the total scattering rate, respectively 4 , All scattering rate components approximately decrease as $\lambda^{-4}$ with increasing photon wavelength $\lambda$.

\section{Absorption}

Photon attenuation in mineral oil, due to either fluorescent emission, scattering, or absorption, was measured at Fermilab with different experimental setups and over a wide photon wavelength range [137]. The difference between the attenuation rate curve as a function of wavelength obtained by these measurements on the one hand, and the sum of the fluorescence and scattering rates discussed above on the other, is interpreted as photon absorption in mineral oil.

\footnotetext{
${ }^{4}$ In this context, (an)isotropic refers to the nature of the density flucutations responsible for the Rayleigh scattering process. Both isotropic and anisotropic Rayleigh scattering are anisotropic, as far as angular distributions are concerned.
} 


\subsubsection{PMT/DAQ Response, and Hit-Level Detector Calibra- tion}

Having described the production and transmission of optical photons to the PMT surface, we are left with the description of the PMT/DAQ charge and time response.

\section{Charge Response}

The charge response can be classified into three parts: photocathode response, charge collection response, and charge digitization. The photocathode response is described by the quantum efficiency, that is the probability to convert one photon of wavelength $\lambda$ into one photoelectron at the photocathode surface. The charge collection efficiency gives the probability, for a photoelectron created at the photocathode surface, to be collected and amplified by the PMT dynode chain. The quantum efficiency, defined here as the phtocathode efficiency times the charge collection efficiency, is shown in Fig. 6.7 as a function of photon wavelength $\lambda$, for both R1408 and R5912 PMTs [139]. For both types of PMTs, the quantum efficiency peaks at around $\lambda=400 \mathrm{~nm}$; moreover, the quantum efficiency of R1408 PMTs is about 15\% lower than the R5912 PMT quantum efficiency over all wavelengths.

The charge collection response is determined by the photocathode geometry (shown in Fig. 6.3), the incident light spatial and angular distribution on the mineral oil/photocathode boundary, and by details of photon and electron transport at the photocathode and through the PMT dynode chain. The charge collection angular response has been measured by immersing the PMTs in mineral oil, and by illuminating their photocathode surface with a broad, parallel beam of light, for various angles $\eta$ between the photon beam and the PMT symmetry axis (head-on light for $\cos \eta=1)$. The results are shown in Fig. 6.7 b, normalized to the $\cos \eta=1$ response. The dominant effect in Fig. 6.7b is the solid angle subtended by the photocathode surface with respect to the beam of light; since R1408 and R5912 PMT photocathode 

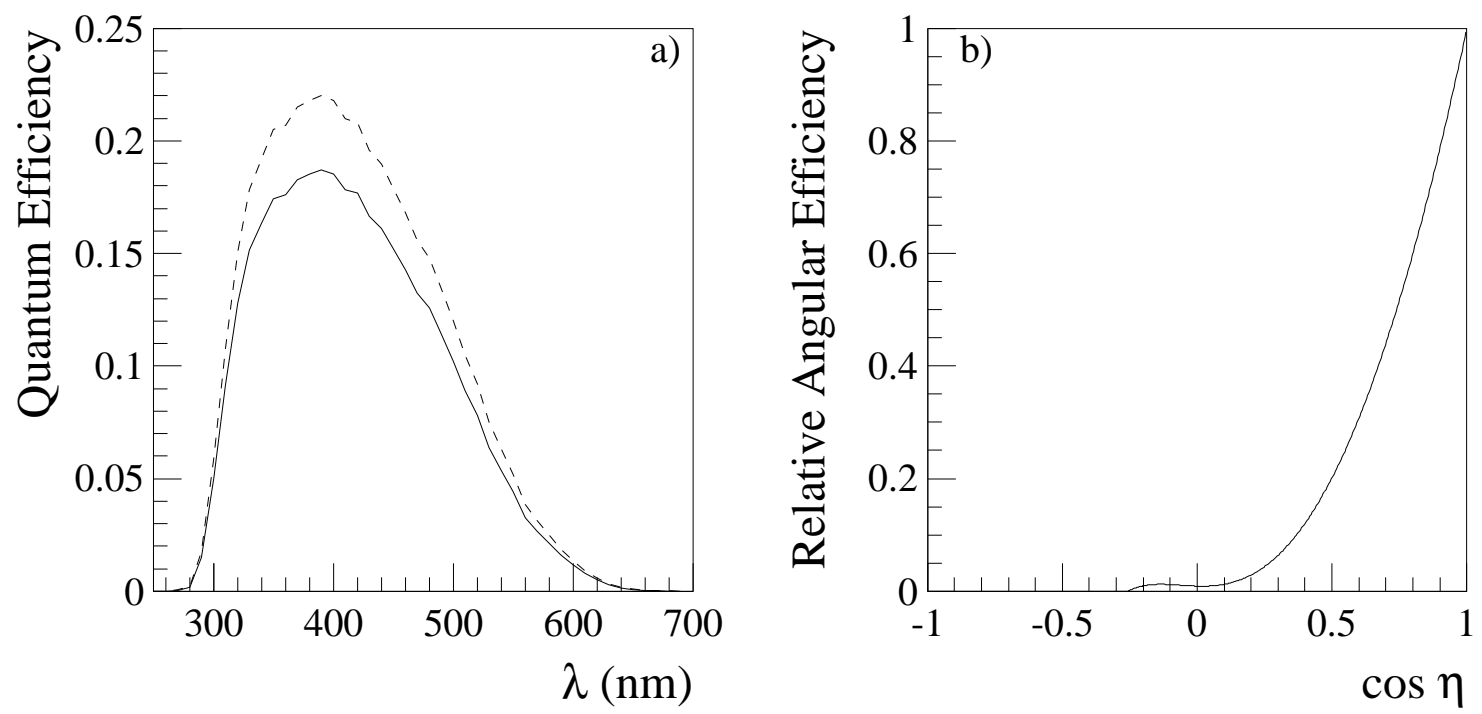

Figure 6.7: Quantum efficiency and angular response for MiniBooNE PMTs. Figure 6.7 a shows the quantum efficiency as a function of incident photon wavelength $\lambda$, for R1408 (solid curve) and R5912 (dashed curve) PMTs. Figure 6.75 shows the angular efficiency for both R1408 and R5912 PMTs, where $\eta$ indicates the angle between the direction of a broad beam of light and the PMT symmetry axis, relative to the efficiency for light hitting the PMTs head-on $(\cos \eta=1)$.

geometry is the same, the curve in Fig. 6.7b is approximately applicable to both types of PMTs.

The details of electron transport through the PMT dynode chain determines the PMT charge resolution, that is the probability to measure an amount of charge $q$ for a given amount $\mu$ of photoelectron charge created at the photocathode. Photomultipliers of type R1408 and R5912 have a different dynode structure, leading to different charge collection responses. The charge resolution is measured via the MiniBooNE laser/flask calibration system, using the charge digitization scheme described in Section 6.1.3. Therefore, the PMT charge calibration relating the charge ADC values to number of photoelectrons is described first.

The charge calibration [140] is extracted by directing very low levels of light to the laser central flask, located at the same distance with respect to all MiniBooNE PMTs. 
The light level and flask/PMT distance chosen ensure that, most of the times, each PMT is hit by at most one photon for every laser light pulse. Small corrections due to multi-PE hits are taken into account in the charge calibration procedure. The PMT charge calibration can be extracted, individually for each PMT, by requiring that $\langle q\rangle=1 \mathrm{PE}$, where the average is intended over a large number of hits with nonzero charge. More specifically, calibrated charges $q$ are extracted from ADC values via:

$$
q[\mathrm{PE}]=\frac{V_{q}[\mathrm{ADC}]}{g_{q}[\mathrm{ADC} / \mathrm{PE}]}
$$

where $g_{q}$ are calibration constants converting ADC counts to number of photoelectrons, while $V_{q}[\mathrm{ADC}]$ is a function using the four digitized charge ADC values to obtain the total PMT integrated charge, in ADC counts. The calibration constants $g_{q}$ are different for each PMT channel, to account for different PMT gains, preamplifier gains, and different PMT dynode structures. The function $V_{q}$ is common to all PMTs of the same type (R1408 or R5912), but different for the two types of PMTs, to account for the different PMT signal shapes.

Having briefly defined the PMT charge calibration procedure, we can discuss the PMT charge resolution on a hit-by-hit basis [141, 142]. The PMT charge resolution plays an important role in the accuracy of the MiniBooNE energy reconstruction. The probability to measure a calibrated charge $q$ given a predicted average amount $\mu$ of charge is shown in Fig. 6.8, for both R1408 and R5912 PMTs, and for average PMT charges of $\mu=5,1$, and 0.1 photoelectrons. The charge resolutions shown in Figs. 6.8 and b, for the typical cases of $\mu=5$ and 1 average photoelectrons, are a convolution of a Poisson distribution in the number of photelectrons, with the intrinsic PMT charge resolution distribution for a given number of photoelectrons. The intrinsic PMT charge resolutions alone for single-photoelectron hits are shown in Fig. 6.8 , corresponding to $\mu=0.1$ average photoelectrons, since multi-PE hits have a negligible impact in this case. The charge likelihood curves in Fig. 6.8 have been zero-suppressed. Typical intrinsic charge resolutions for R1408 (R5912) PMTs are about $0.8(0.5)$ PEs for single-PE hits, where the resolution is defined here as the 

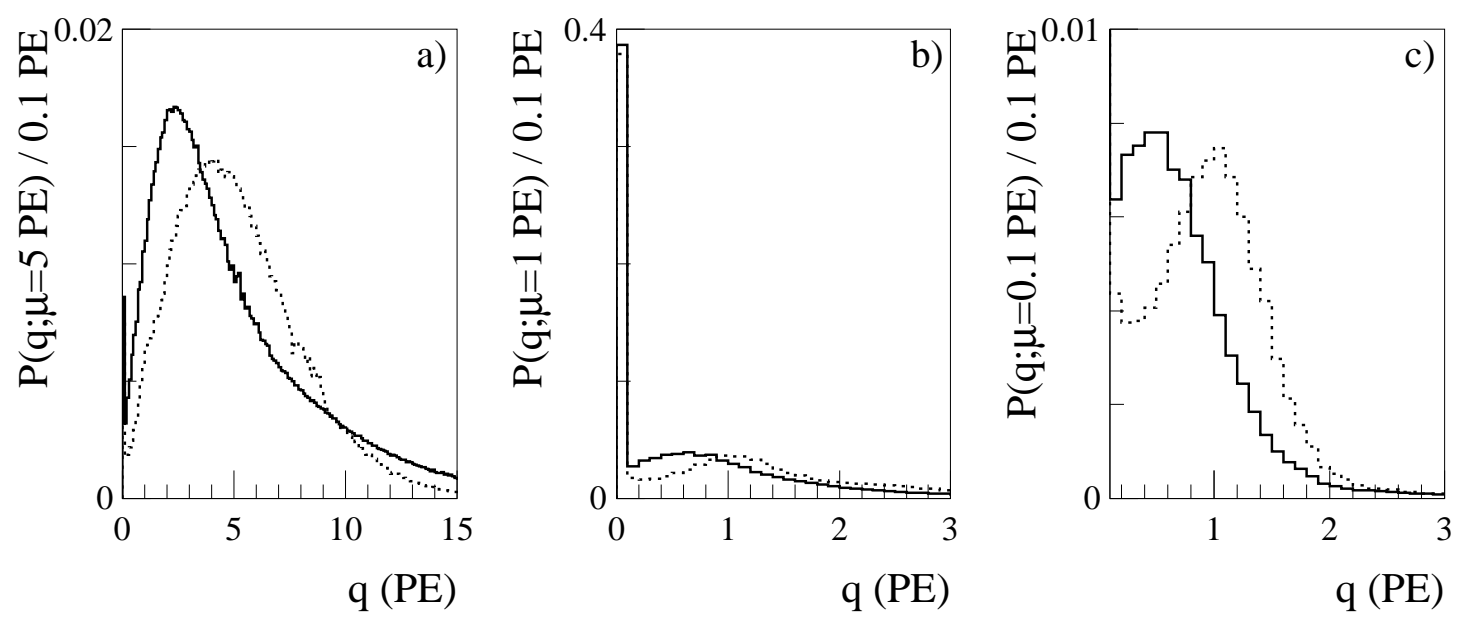

Figure 6.8: Charge response to 5 (panel a), 1 (panel b) ), and 0.1 (panel c) ) average number of photoelectrons $\mu$, for MiniBooNE PMTs. The response of R1408 (LSND) PMTs is shown with solid histograms; the response of R5912 (new) PMTs is shown with dotted histograms. The charge response shown in c) has been zero-suppressed.

RMS of the distributions shown in Fig. 6.8k for $q>0$.

This charge resolution is sufficient for obtaining a satisfactory energy reconstruction, given the large number of PMT hits in a typical neutrino interaction: for example, muon neutrino charged-current, quasi-elastic neutrino interactions reconstructed in MiniBooNE have a mean number of PMT hits in the main detector region of about 500, and a mean charge per PMT hit of about 3 photoelectrons.

The procedure used to extract the charge resolution functions $P(q ; \mu)$ shown in Fig. 6.8 also use the laser/flask system, this time over a wide range of laser light intensity and a wide range of laser flask / PMT distances. The reconstruction algorithm used in this case to predict the amount of PMT charge $\mu$ is based not only on the light transmission (Section 6.2.2) and PMT response (this Section) properties described above, but also on the known laser light production characteristics (wavelength and position of light source, angular distribution of emitted light, etc.). The normalization constant for a given light intensity is determined via $\langle\mu\rangle_{q>0}=\langle q\rangle_{q>0}$, where the average is over all non-zero charge PMT hits. Based on the PMT charge 
prediction, it is possible to predict the fraction of events in which no photocathode charge is created, and therefore extract the full charge resolution function including the $q=0$ case, also shown in the distributions in Fig. 6.8. These histograms include the effects due to the discriminator threshold of approximately $0.25 \mathrm{PE}$, and PMT hits corresponding to a charge below threshold are given in the $q=0$ bin. Two charge resolution functions are obtained with laser data, one for R1408 PMTs, and one for R5912 PMTs. The charge response $P(q ; \mu)$ described above forms the basis of the

charge likelihood part of the MiniBooNE maximum likelihood event reconstruction discussed in Section 6.3.1.

\section{Time Response}

Similarly to the charge response, the time response also depends both on details related to the PMT charge collection mechanisms, and on the DAQ digitization algorithm used in MiniBooNE. Moreover, the time response is also affected by the intrinsic time delays associated with scintillation and fluorescence light production, and scattering. As for the charge response, we start by describing the PMT time calibration, and then discuss the PMT time resolution.

The time calibration for each of the MiniBooNE PMTs, relating time ADC values to charge collection times, can be extracted with laser events [140]. More specifically, calibrated times $t_{\text {corr }}$, corrected for the photon transit time from the light source to the PMT, are obtained via:

$$
t_{\mathrm{corr}}=t_{\mathrm{raw}}\left(g_{t}[\mathrm{ADC} / \mathrm{ns}]\right)+t_{\text {offset }}-t_{\text {slew }}(q)-t_{0}-\left|\vec{x}_{p m t}-\vec{x}_{0}\right| / c_{n}
$$

where the raw time $t_{\text {raw }}$ depends on channel-specific time slopes $g_{t}$ determining the relationship between times in ns and time ADC counts $V_{t}, t_{\text {offset }}$ is a channel-specific time offset accounting for cable length differences and different dynode structures, $t_{\text {slew }}(q)$ is a "first-order" time slewing correction depending on the amount of PMT charge $q, t_{0}$ is the mean emission time of photons from the laser flask source, $\vec{x}_{\mathrm{pmt}}$ is 
the known PMT location, $\vec{x}_{0}$ is the known laser flask location, and $c_{n}$ is the velocity of light in a medium of refractive index $n$. For laser light, the time distribution $F\left(t_{\mathrm{e}}\right)$ of light emission has a negligible time width compared to the detector time resolution, and can be taken to be instantaneous: $F\left(t_{\mathrm{e}}\right)=\delta\left(t_{\mathrm{e}}-t_{0}\right)$.

The corrected time resolution functions for the MiniBooNE PMTs cannot be entirely extracted from laser events, since tracks in the detector produce delayed scintillation light that cannot be simulated with the laser system, and therefore the time distributions of light emission $F\left(t_{\mathrm{e}}\right)$ are different in the two cases. For particle tracks in the detector, whose position is generally unknown prior to reconstruction, the PMT corrected time is still described by Eq. 6.6, where $t_{0}$ and $\vec{x}_{0}$ are now parameters to be determined, referring to the track's mean light emission time, and track position (see Section 6.3.1).

The corrected time resolution functions assumed by the reconstruction algorithm for three different light detection hypotheses are shown in Fig. 6.9. The examples given refer to $50 \mathrm{MeV}$ electron tracks. We mention here three important features related to the expected corrected time distributions shown in Fig. 6.9.

PMT intrinsic time resolution : Figs. 6.9 and 6.9 b, refer to PMTs that are hit, on average, by one photoelectron due to prompt, Cherenkov, light. From these figures, typical PMT intrinsic time resolutions of about 1.2 (1.6) ns for R5912 (R1408) PMTs can be extracted. This reconstruction parameter is tuned based on low intensity laser events, by measuring the spread in the PMT hit times [143]. As discussed in Section 6.3.1, the PMT intrinsic time resolution is important for reconstructing the light source position (laser flask or lightemitting particle track) in the detector, and all the reconstructed parameters that depend on the event position. Based on the velocity of light in mineral oil, a time spread of $1 \mathrm{~ns}$ approximately corresponds to a $20 \mathrm{~cm}$ spread in the reconstructed distance between the light source and the PMT location. 

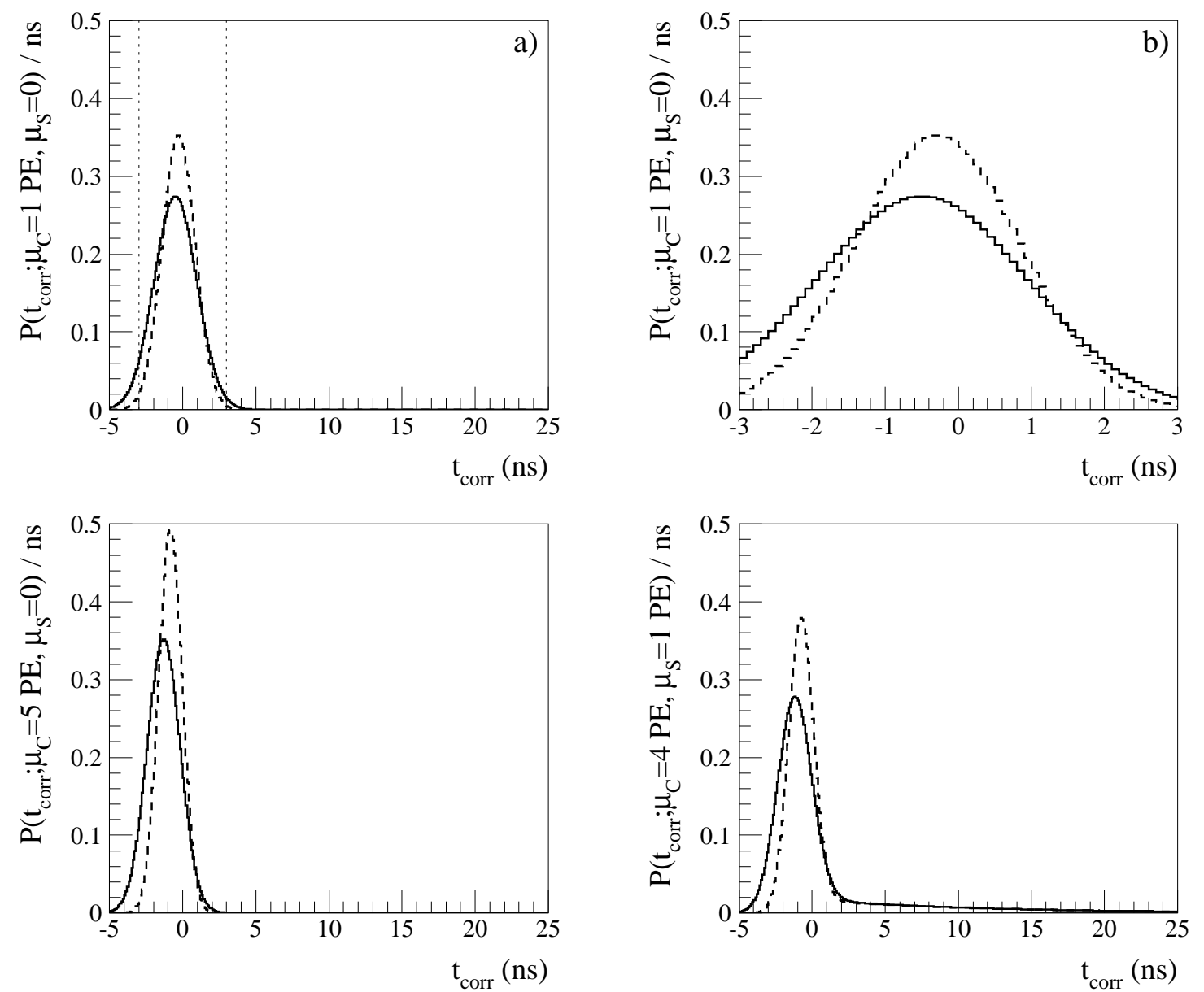

Figure 6.9: PMT corrected time likelihood for $50 \mathrm{MeV}$ electron events, as used in the MiniBooNE reconstruction algorithms. The response function for R1408 (R5912) PMTs is shown by the solid (dashed) histograms. Figure 6.9 a shows the expected corrected time distributions for PMTs that are hit, on average, by a single Cherenkov photon; Figure 6.9b is the same as Fig. 6.9a, but zoomed in the $-3<t_{\text {corr }}(n s)<3$ time interval; Figures 6.9c and 6.9d show the corrected time distributions for PMTs that are hit, on average, by five Cherenkov photons, and by four Cherenkov photons plus one scintillation photon, respectively.

Time slewing and multi-PE hits : the corrected time distributions for an average of $5 \mathrm{PE}$ prompt hits (Fig. 6.9 ) are shifted toward earlier times and are narrower, compared to the $1 \mathrm{PE}$ prompt hits ones (Fig. 6.9 a). This is due to a combination of two effects. First, time slewing, that is the time jitter for a 
PMT signal to cross the DAQ threshold, is more pronounced for low-charge signals [140]. Second, the MiniBooNE DAQ system records the time of the earliest photoelectron only, even when several photoelectrons are present in a hit. Also this second effect tends to decrease the mean time and width of the corrected time distributions [144. This feature is measured with laser events as well, using different light levels and laser flask positions. Therefore, the time likelihood tables include a "second-order" time slewing correction, not included in the "first-order" correction applied in the corrected times definition of Eq. 6.6.

Delayed light: Figure 6.9d, referring to the corrected time distribution expected for PMT signals due to an average of four Cherenkov photoelectrons and one scintillation photoelectron, shows a long exponential tail for large corrected times, with a typical decay time constant of the order of tens of ns. This tail is due to the delayed light emission from scintillation processes. As the time slewing and multi-PE effect, it is coupled to the random, Poisson statistics nature of photon (and photoelectron) production, and to the intrinsic PMT charge resolution. First, there is a non-zero probability that no prompt photoelectrons are actually created at the PMT photocathode, for an average number of four Cherenkov photoelectrons; second, even if Cherenkov photons reach the PMT and are converted into photoelectrons, those can give rise to PMT signals below threshold because of non-zero charge resolution. Therefore, the PMT hit time can be determined by the arrival time of delayed, scintillation photons only. This aspect of the corrected time distribution is tuned based on electron events from muon decays at rest [144]. In this context, the tail at large corrected times is not solely due to scintillation, but also to fluorescence and scattering processes. 
In addition to the ones described above, there are other aspects affecting the corrected time distributions. First, higher-energy particle tracks have a light emission pattern which is spatially more extended, and therefore a broader corrected time distribution. This effect is taken into account by the reconstruction algorithms [144]. Second, PMT pre-pulsing, PMT after-pulsing, and light reflections from the black-painted PMT support structure have been observed with laser events [143], and are taken into account in the detector Monte Carlo simulation; their impact on the reconstruction is expected to be small, and it is neglected in the algorithms that we describe next.

\subsection{Reconstruction and Particle Identification Al- gorithms in MiniBooNE}

\subsubsection{Event Reconstruction}

The maximum likelihood event reconstruction in MiniBooNE [144] uses the measured charge and time PMT responses in the MiniBooNE detector to reconstruct the properties of the final state of a neutrino interaction. The algorithms used focus on the three most important types of final state particles that can be observed with the MiniBooNE neutrino beam: electrons, muons, and neutral pions. Electrons from muon decays are reconstructed separately from the prompt part of the neutrino interaction, since the muon decay timescale (of the order of $2 \mu \mathrm{s}$ ) is much longer than the timescales for light production, propagation and detection in the detector (tens of ns), which ultimately affect the particle reconstruction. The reconstruction categorizes events based on the number of expected Cherenkov rings. Electrons and muons produce a single ring of Cherenkov light in the prompt part of the event (i.e, ignoring muon decay), while $\pi^{0} \rightarrow \gamma \gamma$ decays produce two rings, one for each photon conversion in mineral oil. Events can therefore be reconstructed both under a single ring, and a two-ring assumption. This analysis focuses on events with a single muon in the final 
state, and therefore we discuss below the single Cherenkov ring reconstruction only. Differences between muon and electron/photon Cherenkov rings are briefly discussed in the Section 6.3.3, addressing particle identification. Moreover, there currently exists two independent reconstruction software packages in MiniBooNE; only the one used in this analysis is described below.

For each event with a single Cherenkov track in the final state, the following quantities are reconstructed:

- mean photon emission position and time along the track;

- direction of the track;

- length of the track;

- separate amounts of Cherenkov and scintillation light produced by the track;

- energy of the track.

Given the complexity of the reconstruction algorithms, not all quantities are reconstructed at once. Rather, a step-by-step minimization approach is chosen, in which the sophistication of the model prediction is gradually increased for better reconstruction, and in which the results of the previous minimization step are used as starting point. For muon reconstruction, the four minimization steps used are described below, and summarized in Tab. 6.2. The main building blocks for the time and charge likelihood functions are discussed in Sections 6.2.1, 6.2.2, and 6.2.3, where the emphasis is on hit-level quantities. The reconstruction calibration for global event quantities, such as energy or light fluxes, is discussed in Section 6.3.2.

\section{Step 1: Track Position, Time, Direction, and Energy from Simple Time Likelihood}

First, the approximate position $\vec{x}_{1}$ and time $t_{1}$ of the mid-point of the muon track are reconstructed (4 parameters), using PMT time information only, based on two 
generic time likelihood functions for the corrected times defined in Eq. 6.6, depending on the PMT type, i.e of type R1408 or R5912. As already mentioned, these time likelihoods have two components: a Cherenkov component for the prompt light, and a scintillation component for the delayed light. Second, the track direction $\hat{u}_{1}$ is determined from a charge-weighted average of the directions defined by the PMT locations with respect to the position $\vec{x}_{1}$, using only the prompt charge detected by the PMTs that measure a non-zero charge. Third, the track energy $E_{1}$ is reconstructed, following a parametrization that depends on the total measured charge $Q$ for the event, and the distance of closest approach between the position $\vec{x}_{1}$ and the spherical surface defined by the PMT locations. Fourth, the predicted Cherenkov $(\rho)$ and scintillation $(\phi)$ light fluxes emitted by the track are computed, assuming that both light fluxes are directly proportional to the reconstructed energy $E_{1}$.

\section{Step 2: Track Position, Time, Direction, and Energy, from Refined Time and Charge Likelihoods}

First, the accurate position $\vec{x}_{2}$, time $t_{2}$, and direction $\hat{u}_{2}$ of the track are reconstructed (6 parameters), using both PMT time and charge information, based on two combined time plus charge likelihood functions, one for each PMT type. The time likelihoods used in this minimization step are more sophisticated than the previous ones, and depend on the reconstructed energy $E_{1}$ as well as on the amounts of charge $\mu$ predicted by the reconstruction for each PMT, which in turn depend on the energy $\left(E_{1}\right)$, and on the Cherenkov $\left(\rho_{1}\right)$ and scintillation $\left(\phi_{1}\right)$ light fluxes returned by the first reconstruction step. The dependency of the time likelihood from the predicted charges $\mu$ and track energy $E$ is discussed in Section 6.2.3. The charge likelihoods introduced in this step depend on the same three quantities $E_{1}, \rho_{1}, \phi_{1}$. Unlike the time likelihoods, which use only PMTs measuring a non-zero charge, the charge likelihoods use all PMTs, since the probability to measure no charge $q_{j}$ on a PMT given a certain predicted amount of charge $\mu_{j}$ is a well-defined quantity that can be com- 
puted, as also discussed in Section 6.2.3. Second, using the new position $\vec{x}_{2}$, a more accurate estimate of the energy $\left(E_{2}\right)$ is computed, using the same parametrization of step 1. In this minimization step, the Cherenkov and scintillation light fluxes are kept unchanged.

\section{Step 3: Cherenkov and Scintillation Light Fluxes}

First, new values for the predicted Cherenkov $\left(\rho_{3}\right)$ and scintillation $\left(\phi_{3}\right)$ light fluxes are computed (2 parameters), using combined time plus charge likelihood functions similar to the ones used in step 2. In this minimization step, the midpoint track position, time, and direction are kept fixed to the values obtained in step 2 of the reconstruction (i.e., $\vec{x}_{2}, t_{2}, \hat{u}_{2}$ ). Second, an energy estimate that is alternative to the one computed in step 2 is obtained: the reconstructed energy $E_{3}$ is based solely on the predicted amount of Cherenkov light $\rho_{3}$ in the event. The proportionality constant $C_{\rho}$ between energy and Cherenkov light flux is the same as the one used in step 1.

\section{Step 4: Track Length}

The track extent (1 parameter) is estimated in this last step. Unlike the previous three steps, the measured PMT times and charges are not assumed to be due to a point-like Cherenkov and scintillation light source in the detector. Rather, the reconstruction assumes the simplest generalization to an extended track, that is two point-like sources of light, each having half the Cherenkov and scintillation flux strength previously computed. The two sources are assumed to be distributed along the track direction, and equally spaced with respect to the track midpoint. This "symmetry" assumption is motivated by the nearly uniform light emission along the muon track due to both the Cherenkov and scintillation light processes. As shown in Fig. 6.5, this is a reasonable approximation for the part of the muon track above $p_{\mu}=200 \mathrm{MeV} / \mathrm{c}$. A discrete light emission model based on two point-like sources is assumed (as opposed 
to a continuous light model), to keep the CPU time requirements of the reconstruction algorithm at a manageable level. As for steps 2 and 3, combined time plus charge likelihoods are used in step 4. For the purposes of estimating the track length, the midpoint track position, time, and direction are assumed to be the ones computed in step $2\left(\vec{x}_{2}, t_{2}, \hat{u}_{2}\right)$, while the Cherenkov light flux, scintillation light flux, and track energy are fixed to the values computed in step $3\left(\rho_{3}, \phi_{3}, E_{3}\right)$.

\subsubsection{Reconstruction-Level Detector Calibration and Valida- tion}

We now briefly discuss the calibration and validation of global quantities related to the MiniBooNE events.

The accuracy of the light source position reconstruction can be validated with laser events, since the position of the laser flasks is known; typical accuracies of the order of $20 \mathrm{~cm}$ are obtained [145].

The accuracy of the track direction reconstruction can be validated with cosmic ray muons passing through the muon tracker, whose direction is known. Directional accuracies of the order of $3 \mathrm{deg}$ are obtained [133].

The track length reconstruction can be calibrated and its accuracy validated with cosmic ray muons passing through the muon tracker and stopping in a scintillation cube, whose track length in the detector is known. The reconstructed length tends to be reconstructed to lower values compared to the actual track length, for track lengths exceeding about $2 \mathrm{~m}$. This bias is due to the two-point light source approximation used in determining the track length, which becomes less and less valid as the track length increases, and therefore it is understood. Correcting for this bias, typical accuracies of the order of $10 \%$ are obtained [134.

The energy reconstruction is calibrated with electrons from muon decays at rest, whose energy distribution is known, characterized by an endpoint energy of 52.3 
$\mathrm{MeV}$. More specifically, this sample is used to determine the functional form for the charge-to-energy conversion $E=E(Q, \vec{x})$ appearing in Tab. 6.2. Moreover, the accuracy of the energy reconstruction can be validated with electron, muon, and neutral pion events. First, the energy resolution obtained for electron events from muon decays at rest at the decay endpoint of $52.3 \mathrm{MeV}$ is about $13 \%$ [141. Second, the energy resolution for cosmic ray muons stopping in scintillation cubes is of the order of 10\% [134]. Few percent corrections, based on Monte Carlo studies of electron neutrino and muon neutrino charged current, quasi-elastic events, can also be applied, slightly improving the electron and muon energy reconstruction accuracy [146]. Third, neutral pion invariant mass obtained from the opening angle and energy of the two measured decay photons provides another cross-check on the detector energy scale; typical reconstructed invariant masses are in good agreement with the well-known $\pi^{0}$ mass value [147]. The neutrino energy can be estimated in charged-current, quasielastic interactions from the energy and direction of the outgoing charged lepton. The expected neutrino energy reconstruction accuracy as obtained from Monte Carlo studies is described in Chapter 7, discussing muon neutrino charged-current, quasielastic interactions in MiniBooNE.

Finally, the calibration constants relating the Cherenkov and scintillation light fluxes to energy, that is the constants $C_{\rho}$ and $C_{\phi}$ appearing in Tab. 6.2. are obtained from electrons from muon decays at rest [141].

\subsubsection{Particle Identification}

Particle identification in MiniBooNE aims at distinguishing events with a muon, electron, or a neutral pion in the final state. Qualitatively, muon events are characterized by single, long tracks slowing down and brought to rest in the detector because of ionization energy loss, with little scattering along the path. Electron events are characterized by single, shorter tracks, whose development is affected by electron 
bremsstrahlung and ionization, and by photon pair production and Compton scattering, since typical electron energies (tens to few hundreds of $\mathrm{MeV}$ ) are of the order of the electron critical energy in mineral oil $(\simeq 110 \mathrm{MeV}[60])$. Therefore, in the MiniBooNE energy regime, electrons do not behave neither as minimum ionizing particles, nor as electromagnetic showers, but rather as something in between. The result is that the Cherenkov light ring emitted by electrons is not as filled in as for muon tracks (because of the shorter track lengths), and with a fuzzier ring profile (because of its partially "shower-like" properties). Neutral pion events are characterized by two electron-like Cherenkov rings, from the conversion of the two photons from $\pi^{0} \rightarrow \gamma \gamma$ decays.

For example, electron/muon separation is accomplished using [148]:

- the number of decay electrons in the event;

- functions depending on reconstructed physical observables, for example the ratio of track length to energy;

- charge related quantities, possibly functions of both the reconstructed and predicted PMT charges, or depending only on PMT reconstructed charges, as a function of various topological variables. A first example involving only reconstructed charges is the distribution of charge as a function of the angle between the track direction and the direction defined by the PMT locations with respect to the track midpoint; muon-like tracks are more extended, and tend to have broader distributions in this angular variable. A second example is the distribution of charge per unit track length along the track, assuming that all photons are emitted at an angle equal to the Cherenkov angle with respect to the track direction; muon-like tracks, with a nearly uniform light production per unit track length and sharply-defined Cherenkov rings, tend to have a narrow distribution in this quantity. One example using both reconstructed and predicted charges is the value of the charge likelihood $\mathcal{L}_{q}$ appearing in Tab. 6.2, 
- distributions related to PMT corrected times, such as the time likelihood $\mathcal{L}_{t}$ appearing in Tab. 6.2, possibly as a function of the same topological variables discussed above;

- distributions related to both PMT charges and times, possibly as a function of the same topological variables discussed above.

Several variants on these quantities are used, and the distributions predicted by the reconstruction algorithms under various reconstruction assumptions, are exploited.

The goal of this analysis is to study $\nu_{\mu} n \rightarrow \mu^{-} p$ interactions. As discussed in Chapter 7, the main background to this analysis are muon neutrino, charged-current interactions where a single pion is also produced. Both event topologies have (at least) one muon in the final state and no prompt electrons. As it will be explained in detail later, similar tools to the ones listed above for electron/muon separation are used in this case, but tailored to the different signal/background event classification. 


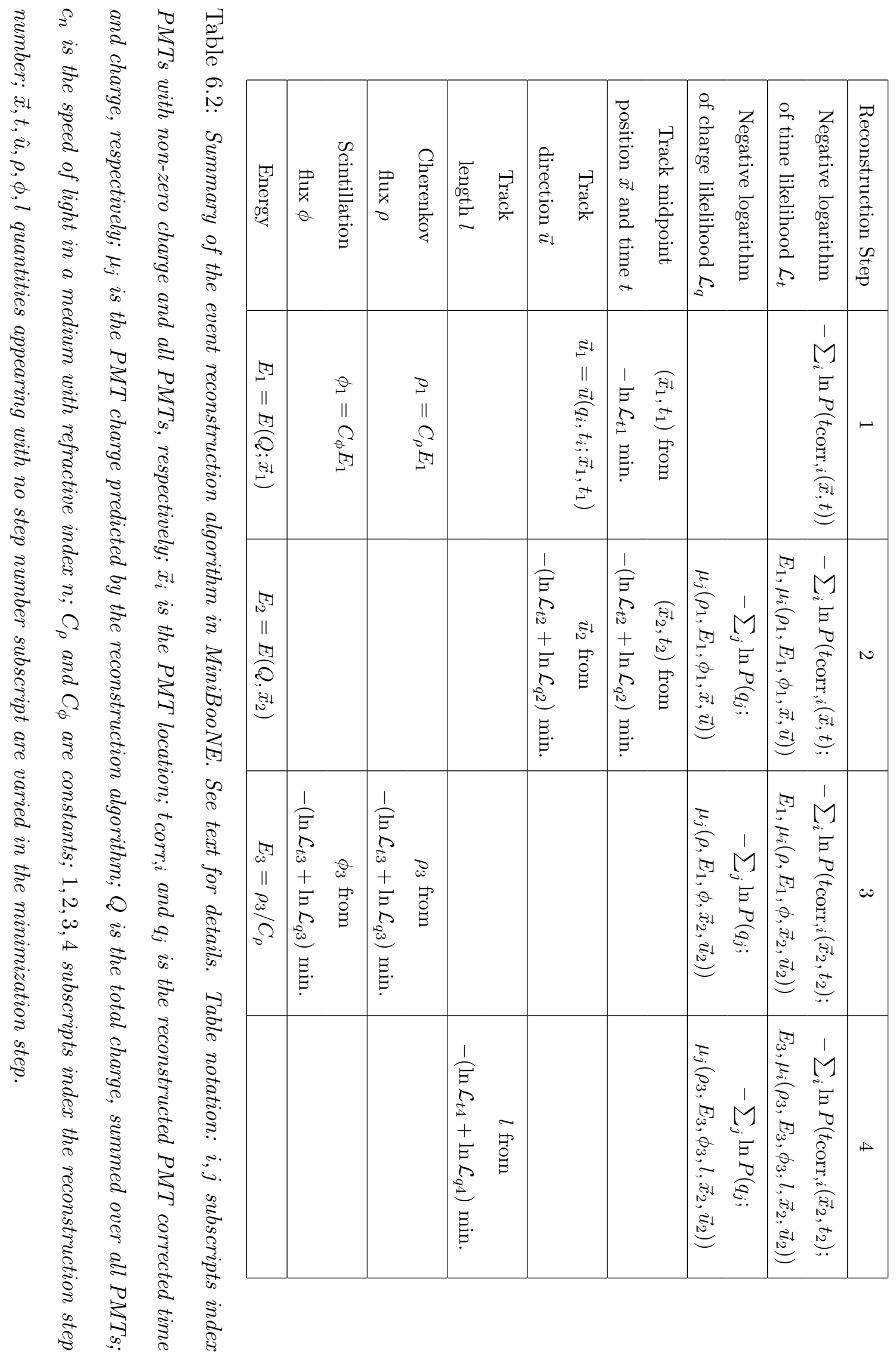




\section{Chapter 7}

\section{Muon Neutrino Charged-current, Quasi-Elastic Interactions in MiniBooNE}

We have discussed the general principles of how to measure neutrino interactions in the MiniBooNE detector. We can now apply these principles to identify the type of neutrino interaction that is most useful for the $\nu_{\mu}$ disappearance analysis, and therefore for searching for sterile neutrinos in MiniBooNE: muon neutrino charged-current, quasi-elastic (CCQE) interaction.

The reason why $\nu_{\mu}$ CCQE interactions are particularly valuable for a $\nu_{\mu}$ disappearance analysis is two-fold. First, neutrino-energy dependent distortions due to oscillations are best measured if the neutrino energy itself can be estimated from the MiniBooNE observables. From the 2-body kinematics in the CCQE reaction $\nu_{\mu} n \rightarrow \mu^{-} p$, the neutrino energy can be determined by measuring the muon energy $E_{\mu}$ and the muon angle with respect to the neutrino beam direction $\theta_{\mu}$. In this case, the only intrinsic smearing effect (in addition to the reconstruction smearing) in the neutrino energy determination is introduced by the motion of the target neu- 
tron within the nucleus, which can be described by a Fermi gas model with Fermi momentum of $p_{F}=220 \mathrm{MeV} / \mathrm{c}$. By correcting for the average neutron momentum and binding energy $E_{B}=25 \mathrm{MeV}$, a neutrino energy determination free of biases and with good resolution can be achieved. Second, a single detector $\nu_{\mu}$ disappearance measurement relies on knowing the neutrino flux and cross-section from external predictions. From this point of view, the best-known neutrino process in the $\simeq 1 \mathrm{GeV}$ energy range is the CCQE interaction.

In principle, a CCQE interaction is strictly defined as the two-body interaction process $\nu_{\mu} n \rightarrow \mu^{-} p$. In practice, as discussed in Section 5.3.3, an irreducible background in a CCQE-enriched sample is present, due to events where a pion is produced in the interaction, and then absorbed in the nuclear environment. Although the interaction-level kinematics is different from the CCQE one, this event category appears, from the experimental point of view, as a CCQE two-body interaction. In the following, we adopt the same definition of Chapter 5 to distinguish $\nu_{\mu} n \rightarrow \mu^{-} p$ interactions ( $C C Q E$ events), from the broader category of CCQElike events. In particular, CCQElike interactions are defined as having only leptons, nucleons, or $<0.1$ $\mathrm{GeV}$ photons in the final state.

\subsection{Description of the Event Selection and Neu- trino Energy Reconstruction}

The $\nu_{\mu}$ CCQE event selection in MiniBooNE relies on three types of cuts applied at various levels of the reconstruction, listed here in increasing order of complexity: basic hit information, event reconstruction, and event type selection. The hit-level and reconstruction-level selection is discussed in Section 7.1.1, the event-level selection is presented in Section 7.1.2. Section 7.1.3 describes how the neutrino energy is inferred from this resulting CCQE-enriched sample. 


\subsubsection{Hit-Level and Reconstruction-Level Selection}

At the basic-hit level, veto hit multiplicity, tank hit multiplicity, and tank hit time cuts are used to reject mostly cosmic ray-induced events with significant veto activity, uncontained neutrino-induced events, neutral current neutrino elastic scatters, and events with more than one muon decay in the final state. The hit-level cuts are applied on the number of subevents within a MiniBooNE neutrino event, as well as on the hit properties of the first subevent. As mentioned in Chapter 3 , a subevent is defined as a collection of PMT hits clustered in time. The main motivation for introducing the concept of subevents is to separate the light emitted by the charged tracks produced in the initial neutrino interaction, from the light produced by the Michel electrons from muon decays at rest. The reason is that the timescale for a muon to decay, set by the muon lifetime of $\tau \simeq 2 \mu s$, is much longer than the timescale for photon production, propagation, and detection in the MiniBooNE detector, which are of the order of $\simeq 10 \mathrm{~ns}$. Therefore, it is possible to apply reconstruction algorithms individually to these two, well separated in time, neutrino-induced sources of light. Specifically, the algorithm used to associate hits to a subevent in MiniBooNE requires that there be at least ten hits per subevent (corresponding to an energy of about $4 \mathrm{MeV}$ ), and such that no two consecutive PMT hits in the subevent are separated by more than $10 \mathrm{~ns}$. Figure 7.1 shows how the splitting of PMT hits into subevents works in practice, for a typical 2 subevent, CCQE candidate event observed in MiniBooNE.

Having defined what subevent means in MiniBooNE, we can now list the hit-level requirements for the $\mathrm{CCQE}$ event selection:

1. NSubEvent $>0$ : at least one subevent;

2. NVeto $<6$ : less than 6 PMT hits in the veto region associated with the first subevent;

3. NTank > 100: more than 100 PMT hits in the main tank region for the first 

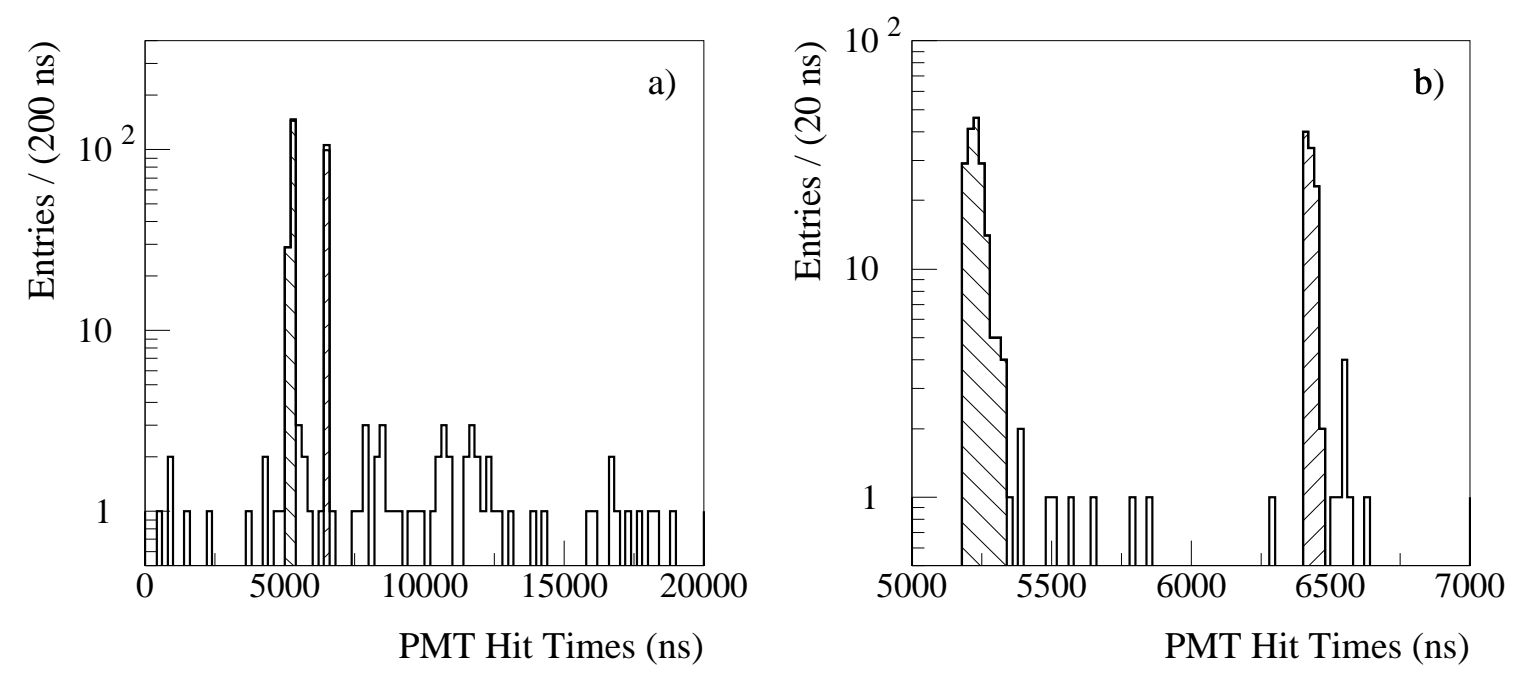

Figure 7.1: Measured PMT hit times distribution for a typical CCQE candidate event in MiniBooNE, and subevent selection. The left-leaning (right-leaning) hatches show the PMT hit times associated with the first (second) subevent, and the non-hatched histogram show all PMT hit times for the event. Figures 7.1 a) and b) show the hit time distribution over the full 19.2 $\mu$ s data acquisition time window and over $2 \mu s$, respectively.

subevent;

4. $4.4<$ TTank $(\mu s)<6.4$ : the time of PMT hits averaged over all first subevent hits is consistent with the time expected for beam-induced neutrino interactions, corresponding to roughly $4.6<$ TTank $(\mu s)<6.2$;

5. NSubEvent $<3$ : no more than two subevents are present in the event.

The reconstruction algorithms summarized in the previous Chapter are then applied to the first subevent. At the event-reconstruction level, successful reconstruction of the event (vertex, time, visible energy, relative fractions of Cherenkov and scintillation light, and event spatial extent) is required, and a fiducial volume cut on the reconstructed fitted Cherenkov track in the first subevent is applied. Specifically, the fiducial volume cut is applied on the radial position of the reconstructed mean light emission point, roughly corresponding to the mid-point of the muon produced in CCQE interactions, which is required to be within $500 \mathrm{~cm}$ from the detector center. 
The two reconstruction-level requirements listed above are referred in the following as NRec $>0$ and $R_{\mu}<500 \mathrm{~cm}$, respectively. The reconstruction cuts are used to reject uncontained and/or poorly-reconstructed neutrino-induced interactions, as in these cases the energy of the neutrino inducing the event cannot be reliably reconstructed.

\subsubsection{Fisher Discriminant Method and Event-Level Selec- tion}

The selection at the event-type level relies on a Fisher discriminant method to isolate events with a single, muon-like Cherenkov ring in the final state and scintillation light production consistent with a $\nu_{\mu} p \rightarrow \mu^{-} n$ interaction. We start by describing the linear discriminant method introduced by Fisher, and will then proceed to apply it in order to isolate CCQE interactions in MiniBooNE.

The Fisher discriminant method is used to construct the linear combination among a set of variables $\vec{x}=x_{1}, \ldots, x_{n}$ with the best discriminating power between two populations. In our case, we aim to discriminate between CCQE and non-CCQE interactions in MiniBooNE, and the variables used to form the most effective linear combination are $n=10$ observables related to particle and event identification properties. Specifically, the Fisher linear discriminant method chooses the coefficients $\vec{a}=a_{1}, \ldots, a_{n}$ in the linear combination:

$$
t(\vec{x})=\sum_{i}^{n} a_{i} x_{i}=\vec{a}^{T} \vec{x}
$$

such that the following measure of separation between two populations $H_{0} \equiv H_{\mathrm{CCQE}}$, $H_{1} \equiv H_{\overline{\mathrm{CCQE}}}$ is the largest:

$$
J(\vec{a})=\frac{\left(\tau_{0}-\tau_{1}\right)^{2}}{\Sigma_{0}^{2}+\Sigma_{1}^{2}}
$$


where the means $\tau_{k}$ and variances $\Sigma_{k}^{2}$ of the linear combination of the two populations $k=0,1$ are given by:

$$
\begin{array}{r}
\tau_{k}=\int d \vec{x} t(\vec{x}) f\left(\vec{x} \mid H_{k}\right) \\
\Sigma_{k}^{2}=\int d \vec{x}\left(t(\vec{x})-\tau_{k}\right)^{2} f\left(\vec{x} \mid H_{k}\right)
\end{array}
$$

where the $2 n$ functions $f\left(\vec{x} \mid H_{k}\right)$ are probability density functions: $\int d \vec{x} f\left(\vec{x} \mid H_{k}\right) \equiv 1$. With this definition for the measure of separation between two populations, the best choice of the coefficients $\vec{a}$ in the Fisher variable $t(\vec{x})$ is the one given by the solution to the following set of $n$ linear equations:

$$
\frac{\partial J}{\partial a_{i}}=0, i=1, \ldots, n
$$

The ten observables used as inputs to the CCQE Fisher algorithm are related to the coarse and fine hit timing structure, and to the spatial topology of the light emission. These ten quantities are chosen empirically, via Monte Carlo studies, in order to obtain maximum separation between CCQE and non-CCQE interactions. The variables are described below, together with the physics motivation for their CCQE / non-CCQE interaction discriminating power:

- NSubEvent: in addition to the $0<\mathrm{NSubEVent}<3$ hit-level cut, the discrete variable NSubEvent $=1,2$ is used as one of the Fisher inputs. The reason is that most CCQE events have 2 subevents due to the Michel decay of the $\mu^{-}$ produced by the $\mathrm{CC} \nu_{\mu}$ interaction, while a significant part of non-CCQE events passing the hit-level and reconstruction-level cuts are neutral-current, resonant $\pi^{0}$ production interactions $\left(\nu_{\mu} N \rightarrow \nu_{\mu} N \pi^{0}\right)$ which generally reconstruct as 1 subevent events;

- VeryPromptHitsFraction and VeryLateHitsFraction: variables measuring the amount of very prompt, Cherenkov-induced light, and very late, scintillationinduced light in the first subevent, respectively. Charged-current, quasi-elastic 
and non-CCQE events generally have different relative contributions of light production from Cherenkov and scintillation processes, because of the different final states of the interactions. For example, background due to neutral current, elastic neutrino interactions $\left(\nu_{\mu} N \rightarrow \nu_{\mu} N\right)$ generally has a large value of very late light fraction compared to CCQE events, since most of light produced in this case is due to delayed, scintillation light;

- TransverseHitsFraction, BackwardHitsFraction, RingSharpness, RingChargeRatio1, RingChargeRatio2: these five variables are related to the detailed spatial hit and charge topology of the event, and measure both the amount of hits and charge contained in the fitted Cherenkov ring under the single ring hypothesis per event used by the reconstruction algorithms, and the level of ring sharpness. For example, the RingChargeRatio1 variable is the fraction of how much charge is contained in the single Cherenkov ring assumption, compared to the total amount of charge in the event. This quantity is expected to be close to unity for CCQE events, since the light produced by the recoil proton in the interaction is generally small. On the other hand, other event types such as $\nu_{\mu} p \rightarrow \mu^{-} p \pi^{+}$are expected to have, on average, a smaller value for this quantity;

- TrackDifference: this quantity measures the difference between a Cherenkovbased and a calorimetric-based estimate of the muon track length in the event. The Cherenkov-based estimate uses the muon track extent obtained from the Cherenkov ring fitting algorithm, while the calorimetric estimate uses the total visible energy in the event divided by the energy loss per unit track length for a minimum-ionizing particle in oil. The difference tends to be larger for CCQE events than for non-CCQE events, since a long, energetic muon track is generally present in CCQE events; 

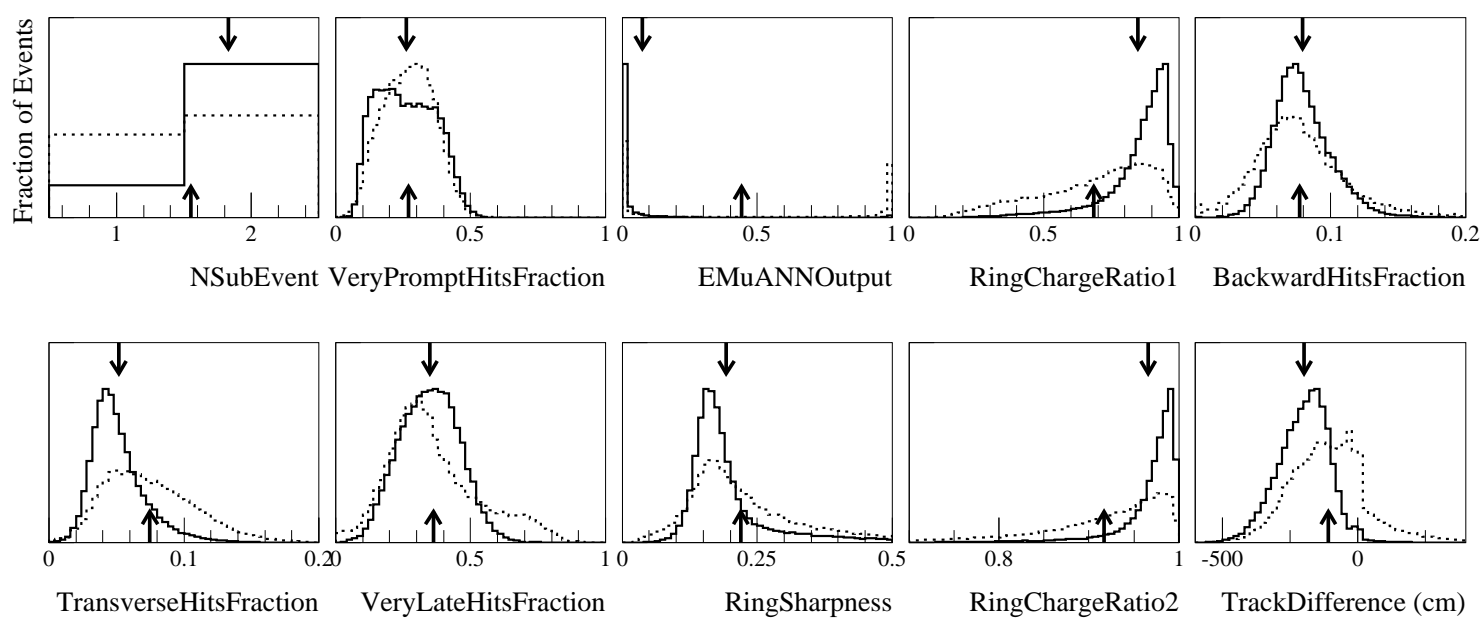

Figure 7.2: Predicted distributions for the ten variables used as inputs to the Fisher discriminant method to identify CCQE interactions. The solid (dashed) distribution refer to CCQE (non-CCQE) interactions passing the hit-level and reconstruction-level cuts. The arrows on top (bottom) show the locations of the means of the CCQE (non-CCQE) distributions.

- EmuANNOutput: output of an artificial neural network discriminant, constructed to separate $\nu_{\mu} \mathrm{CCQE}$ events from $\nu_{e} \mathrm{CCQE}$ events. The output is normalized between zero and one, so that output values close to zero refer to $\nu_{\mu}$ CCQElike events. Because of the MiniBooNE neutrino flux flavor composition, $\nu_{e}$ interactions do not constitute a significant background to $\nu_{\mu}$ CCQE events; however, the particle ID variables used in this network are also effective in distinguishing $\nu_{\mu} \mathrm{CCQE}$ from $\nu_{\mu}$ non-CCQE events. The fifteen particle identification variables used by the network are also related to detailed properties of the time and spatial distribution of hits and charge in the detector. For example, $\nu_{\mu} N \rightarrow \nu_{\mu} N \pi^{0}$ events tend to look more $\nu_{e}$ CCQElike than $\nu_{\mu}$ CCQE-like according to the output of this neural network, since the decay $\pi^{0} \rightarrow \gamma \gamma$ produces two electron-like Cherenkov rings, as opposed to muon-like rings.

Figure 7.2 shows the distributions for the ten observables used in the event-level selection, for all CCQE and all non-CCQE events passing the hit-level and reconstruction- 


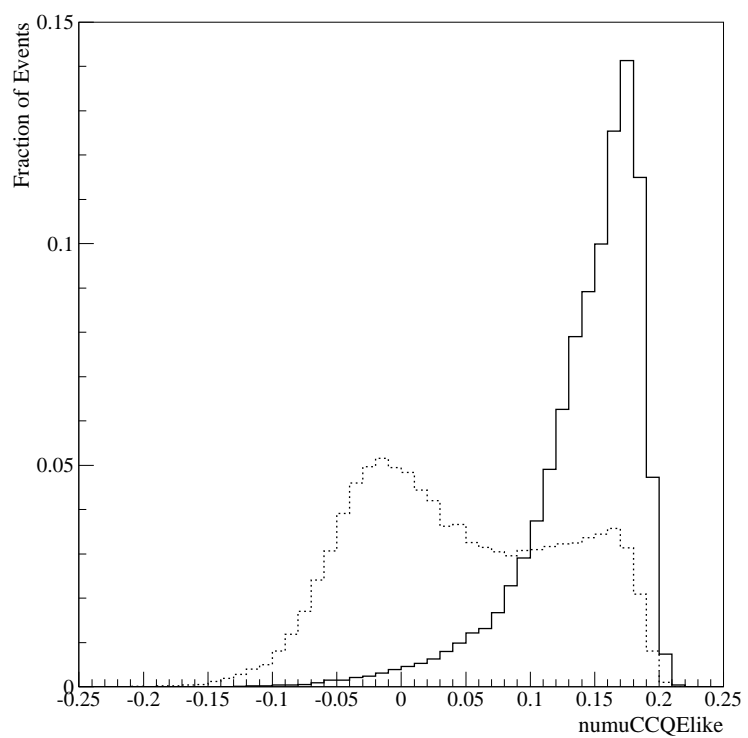

Figure 7.3: Predicted distributions of the Fisher variable numuCCQElike for CCQE (solid) and non-CCQE (dashed) neutrino interactions in MiniBooNE, for events passing hit-level and reconstruction-level cuts.

level selection. The CCQE and non-CCQE distributions for the linear combination of these variables that allow for the best discriminating power between CCQE and non-CCQE (as defined in the Fisher method) is given in Fig. 7.3. This linear combination is called numuCCQElike in the following.

In Section 7.2, we discuss the results of Monte Carlo studies meant to address the performance of the CCQE event selection. The figures of merit are: overall efficiency in selecting CCQE events, CCQE purity of the selected events, energy-dependence of the CCQE efficiency and purity, and resolution in reconstructing the neutrino energy. We first describe the procedure used to reconstruct the neutrino energy.

\subsubsection{Description of the Neutrino Energy Reconstruction Method}

The reconstructed neutrino energy $E_{\nu}^{Q E}$ in a CCQE interaction is obtained from the reconstructed muon energy $E_{\mu}$ and angle $\theta_{\mu}$ between the muon and the neutrino 
direction, via:

$$
E_{\nu}^{Q E}=\frac{1}{2} \frac{2\left(M-E_{B}\right) E_{\mu}+\left(2 M E_{B}-m_{\mu}^{2}+E_{B}^{2}\right)}{\left(M-E_{B}\right)-E_{\mu}+\sqrt{E_{\mu}^{2}-m_{\mu}^{2}} \cos \theta_{\mu}}
$$

where $M$ is the nucleon mass, $m_{\mu}$ the muon mass, and $E_{B}$ is the (positive) binding energy.

In practice, biases and smearing effects due to the non-perfect reconstruction in the muon energy $E_{\mu}$, direction $\theta_{\mu}$, and due to the target nucleon motion, affect the neutrino energy reconstruction. Therefore, a 4-step, empirical calibration based on simulated data is applied to mitigate these effects [146]. The starting point of the neutrino energy calibration procedure are the track energy and direction extracted by the second step in the event reconstruction algorithm described in Chapter 6 .

Step 1: a first-order correction to the reconstructed track energy $E_{v i s} \equiv T_{\mu, 0}$ is applied to obtain the reconstructed muon kinetic energy $T_{\mu, 1}$. The two calibration parameters are obtained from simulated data, by fitting the profile histogram $T_{\mu}^{g e n}$ versus $T_{\mu, 0}$, where $T_{\mu}^{g e n}$ is the generated muon kinetic energy;

Step 2: use Eq.7.6 to compute the reconstructed neutrino energy, with $E_{\mu}$ in Eq.7.6 given by $T_{\mu, 1}+m_{\mu}$, and $\cos \theta_{\mu}$ is the uncorrected value given by the reconstruction algorithm. Based on this neutrino energy estimate $E_{\nu, 2}^{Q E}$, the fourmomentum transfer to the lepton in the interaction is computed, via:

$$
Q^{2}=2 E_{\nu}^{Q E} E_{\mu}\left(1-\beta_{\mu} \cos \theta_{\mu}\right)-m_{\mu}^{2}
$$

where $\beta_{\mu}=\left|\vec{p}_{\mu}\right| / E_{\mu}$;

Step 3: apply a $Q^{2}$-dependent, third-order correction to the neutrino energy $E_{\nu, 2}^{Q E}$. The corrected neutrino energy $E_{\nu, 3}^{Q E}$ is obtained by fitting the profile histogram $\left(E_{\nu, 2}^{Q E}-E_{\nu}^{g e n}\right)$ versus $Q^{2}$

Step 4: finally, a second-order correction to $E_{\nu, 3}^{Q E}$ is applied to obtain the final neutrino energy $E_{\nu, 4}^{Q E} \equiv E_{\nu}^{Q E}$, from a $\left(E_{\nu, 3}-E_{\nu}^{g e n}\right)$ versus $E_{\nu}^{g e n}$ profile histogram. 


\begin{tabular}{|c|rrr|rrr|rrr|}
\hline Cut & \multicolumn{3}{|c|}{ Number of Events } & \multicolumn{3}{c|}{$\epsilon$} & \multicolumn{3}{c|}{ relative $\epsilon$} \\
\hline Event Type & \multicolumn{1}{|c|}{ All } & CCQE & $\overline{\text { CCQE }}$ & All & CCQE & $\overline{\text { CCQE }}$ & All & CCQE & $\overline{\text { CCQE }}$ \\
\hline \hline none & 400,000 & 158,261 & 241,739 & 1.00 & 1.00 & 1.00 & 1.00 & 1.00 & 1.00 \\
\hline NSubEvent $>0$ & 364,153 & 151,366 & 212,787 & 0.91 & 0.96 & 0.88 & 0.91 & 0.96 & 0.88 \\
NVeto $<6$ & 224,253 & 83,997 & 140,256 & 0.56 & 0.53 & 0.58 & 0.62 & 0.55 & 0.66 \\
NTank $>100$ & 170,859 & 79,230 & 91,629 & 0.43 & 0.50 & 0.38 & 0.76 & 0.94 & 0.65 \\
$4.4<$ TTank $(\mu s)<6.4$ & 170,759 & 79,158 & 91,601 & 0.43 & 0.50 & 0.38 & 1.00 & 1.00 & 1.00 \\
NSubEvent $<3$ & 139,066 & 75,048 & 64,018 & 0.35 & 0.47 & 0.26 & 0.81 & 0.95 & 0.70 \\
\hline NRec $>0$ & 138,896 & 74,956 & 63,940 & 0.35 & 0.47 & 0.26 & 1.00 & 1.00 & 1.00 \\
R $\mu$ < $500 \mathrm{~cm}$ & 114,589 & 62,208 & 52,381 & 0.29 & 0.39 & 0.22 & 0.82 & 0.83 & 0.82 \\
\hline numuCCQElike $>0.14$ & 47,605 & 38,946 & 8,659 & 0.12 & 0.25 & 0.04 & 0.42 & 0.63 & 0.17 \\
\hline
\end{tabular}

Table 7.1: Predicted effect of the individual and combined CCQE selection cuts on all neutrino interactions in the $6.1 \mathrm{~m}$ radius spherical MiniBooNE detector, and on CCQE and non-CCQE interactions separately. The number of simulated events are given, as well as the combined cut efficiencies ( $\epsilon$ ) and the individual cut efficiencies (relative $\epsilon$ ).

The data sample used in this energy calibration procedure is given by simulated CCQE interactions passing the hit-level and reconstruction-level cuts described in the previous Section. Once calibration constants are obtained, the same energy correction procedure is applied to actual data. The expected performance of the MiniBooNE neutrino energy reconstruction is presented in Section 7.2. In Section 7.3, electron and muon calibration samples are used to compare the energy reconstruction in real and simulated data, in order to validate the corrections to the energy reconstruction obtained from simulated data. 


\subsection{Predicted Event Selection and Neutrino En- ergy Reconstruction Performance}

\subsubsection{Efficiency and Purity in Selecting CCQE Interactions}

Table 7.1 summarizes the predicted performance of the CCQE event selection in terms of overall CCQE efficiency and purity. The effect of each of the hit-level, reconstruction-level, and event-level cuts on the expected CCQE efficiency and purity is given sequentially for each cut. The efficiency numbers assume neutrino interactions of all types generated uniformly within the full detector radius of $610 \mathrm{~cm}$, in order to quantify the effects associated with the muon track length, veto inefficiency, and resolution in the neutrino interaction vertex. Given this convention, most of the low (39\%) CCQE efficiency of the simple hit-level and reconstruction-level cuts is due to neutrino interactions outside the fiducial volume, $R_{\nu} \gtrsim 500 \mathrm{~cm}$. Ignoring the muon path length and assuming a perfect detector, one would expect a $(500 / 610)^{3} \simeq 55 \%$ efficiency associated with the fiducial volume cut alone. More quantitatively, this is shown in Tab. 7.1 by the low relative efficiencies associated with the veto hit multiplicity, main tank hit multiplicity, and fiducial volume cuts. Moreover, no inefficiencies due to cosmic ray events in the detector are considered in Tab. 7.1; the impact of cosmic ray events on the CCQE event selection is discussed in Chapter 8 . Adding the event-level Fisher cut numuCCQElike $>0.14$ is expected to increase the CCQE purity of the sample from $54 \%$ to $82 \%$, at the cost of reducing the absolute CCQE efficiency from $39 \%$ to $25 \%$. The CCQE purity is defined as the number of CCQE events in the selected sample, divided by the total number of selected events.

The efficiency $\epsilon_{\text {Fisher }}$ of the cut numuCCQElike $>$ numuCCQElike $_{\min }$ as a function of the cut position numuCCQElike $\min _{\min }$ is shown in Fig. 7.4, together with the corresponding CCQE purity, $\eta_{\mathrm{CCQE}}$. The CCQE purity increases roughly linearly by increasing the value of the cut position numuCCQElike min $_{\text {, }}$, but the efficiency drops 

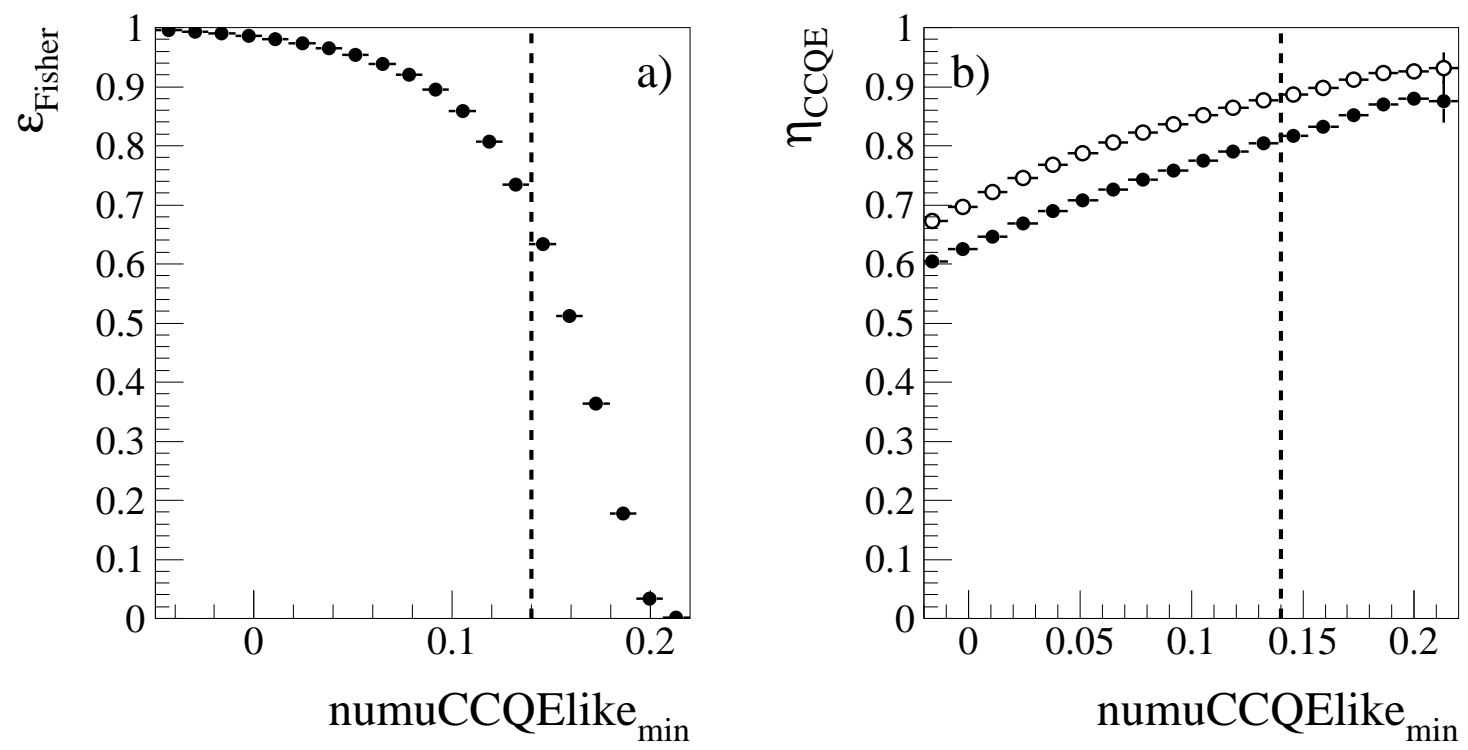

Figure 7.4: Figure 7.4a) shows the predicted efficiency of the Fisher CCQE cut alone, as a function of the minimum Fisher variable value numuCCQElike ${ }_{\text {min }}$, for CCQE interactions passing hit-level and reconstruction-level cuts. Filled points in Fig. 7.4 b) show the predicted

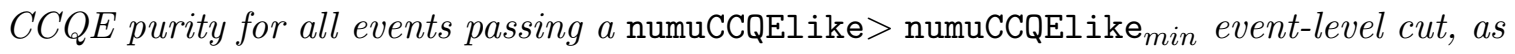
a function of numuCCQElike min; empty points in Fig. 7.4b) show the CCQElike purity, as defined in the text.

very quickly for values numuCCQElike $\min >0.14$. For all cut values, the CCQElike purity is higher than the CCQE purity, given the more inclusive definition of the former category. The impact of the cut value numuCCQElike $\min _{\text {on }}$ the muon neutrino disappearance analysis presented in Chapter 8 has been studied, and showed that a value numuCCQElike $e_{\min } \simeq 0.14$ optimizes the sensitivity to neutrino oscillations. Figure $7.4 \mathrm{~b}$ ) attempts also to give a measure of the level of irreducible background associated with the CCQE event selection, by showing the CCQElike purity. As previously discussed, a significant fraction of non-CCQE neutrino interactions appear as CCQE interactions because of final state interactions within the target nucleus. From Fig. 7.4 b), we expect a $88 \%$ CCQElike purity in the sample by applying a numuCCQElike $>0.14$ cut.

Table 7.2 shows what are the most important backgrounds that are predicted to 


\begin{tabular}{|c|r|r|}
\hline Reaction & \multicolumn{2}{|c|}{$\overline{\text { CCQE }}$} \\
& Contribution (\%) & $\begin{array}{c}\text { CCQElike } \\
\text { Contribution (\%) }\end{array}$ \\
\hline$\nu_{\mu} p \rightarrow \mu^{-} p \pi^{+}$ & 56.9 & 44.1 \\
$\nu_{\mu} n \rightarrow \mu^{-} p \pi^{0}$ & 13.9 & 16.4 \\
$\nu_{\mu} n \rightarrow \mu^{-} n \pi^{+}$ & 12.3 & 13.4 \\
$\nu_{\mu} A \rightarrow \mu^{-} \pi^{+} A$ & 8.0 & 3.9 \\
$\nu_{\mu} n \rightarrow \mu^{-} p$ & 0 & 15.5 \\
other & 8.9 & 6.7 \\
\hline
\end{tabular}

Table 7.2: Predicted backgrounds in the CCQE-enriched sample, given in terms of fractional contributions to the total background. The fractions for two different background definitions are given here: non-CCQE events, and non-CCQElike events (see text for definitions).

be present in the CCQE sample. Two different background definitions are given in Tab. 7.2 non-CCQE background, that is all neutrino interactions that are not of the type $\nu_{\mu} n \rightarrow \mu^{-} p$, and non-CCQElike background, as defined above. The dominant contamination in the CCQE sample is due to $\nu_{\mu} p \rightarrow \mu^{-} p \pi^{+}$events, according to both definitions.

Having discussed the overall efficiency and purity expected in the CCQE sample, we now turn to the third and fourth figures of merit mentioned in Section 7.1.2, that is the energy dependence of these quantities. A selection efficiency that is steeply varying with neutrino energy should be avoided, since even small deviations from its proper characterization may cause significant distortions in the predicted neutrino energy spectrum, possibly mimicking the effect of a neutrino oscillation signal. Similarly, a CCQE purity that varies relatively mildly with neutrino energy is desirable, such that imprecise characterizations of the overall non-CCQE background contamination would not result in gross systematic effects on the energy distribution shape.

Figure 7.5 shows the hit-level and reconstruction-level efficiency $\epsilon_{\text {hit }} \epsilon_{\text {rec }}$, the event-level efficiency $\epsilon_{\text {Fisher }}$, and the combined hit-level, reconstruction-level, and event-level efficiency $\epsilon_{C C Q E} \equiv \epsilon_{\text {hit }} \epsilon_{\text {rec }} \epsilon_{\text {Fisher }}$ as a function of generated neutrino energy, $E_{\nu}^{g e n}$. The CCQE and CCQElike purities of the numuCCQElike>0.14 sample 

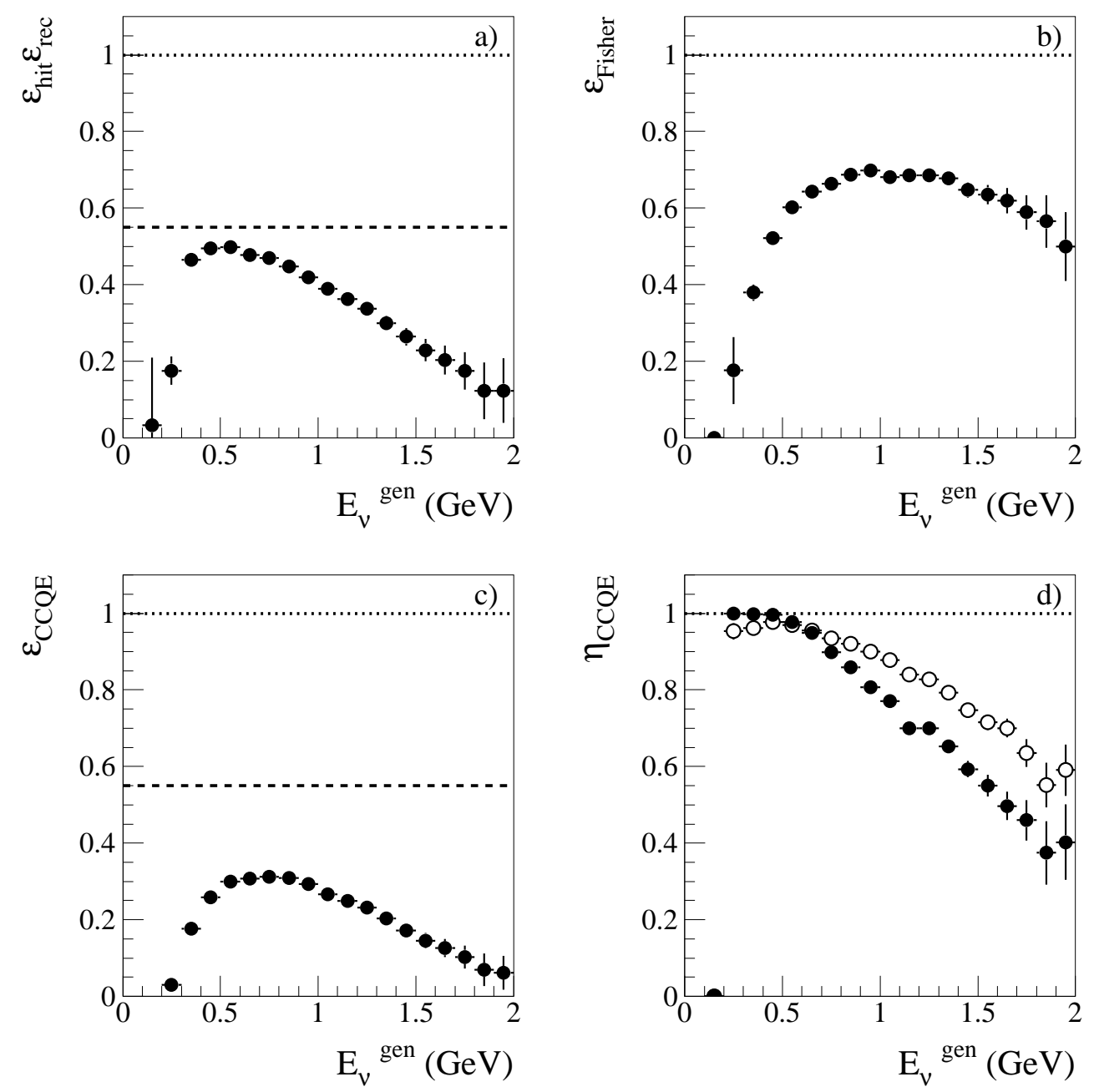

Figure 7.5: Predicted performance of CCQE event selection in MiniBooNE, as a function of true neutrino energy $E_{\nu}^{\text {gen }}$. Figure 7.5 a) shows the efficiency of the hit-level and reconstruction-level cuts, Fig. 7.5b) the efficiency of the Fisher cut for events passing the hit-level and reconstruction-level cuts, and Fig. 7.5c) the efficiency of the the hit-level cuts, reconstruction-level cuts, and Fisher cut combined, for CCQE interactions in the $6.1 \mathrm{~m}$ detector radius. Figure 7.5d) shows the CCQE (solid points) and CCQElike (empty points) purity as a function of $E_{\nu}^{g e n}$.

as a function of $E_{\nu}^{g e n}$ are also given in Fig. 7.5. The efficiencies show a broad peak where the bulk of CCQE interactions are expected, for $0.3<E_{\nu}^{g e n}(G e V)<1.5$. The dashed line in Figs. 7.5a) and c) show the ideal efficiency case discussed above, for $(500 / 610)^{3} \simeq 0.55$. The drop at low energies is mostly due to the main tank hit 

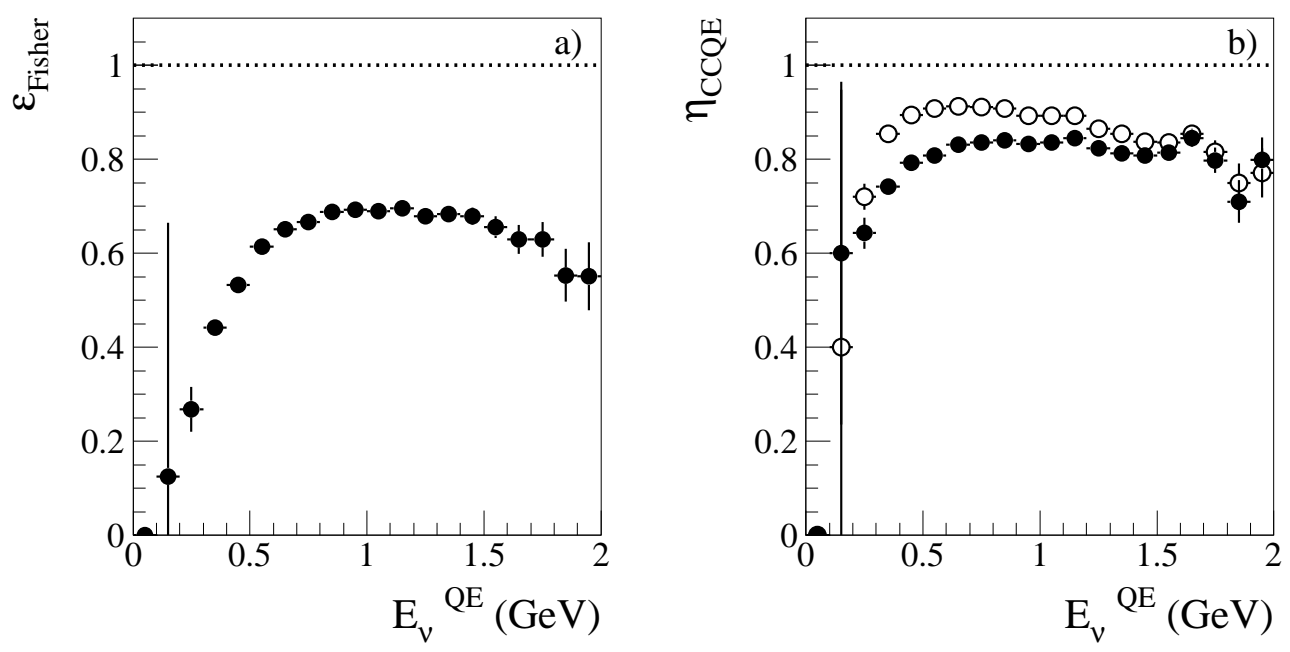

Figure 7.6: Predicted performance of CCQE event selection in MiniBooNE, as a function of reconstructed neutrino energy $E_{\nu}^{Q E}$. Figure 7.6 a) shows the Fisher cut efficiency for CCQE interactions, as a function of $E_{\nu}^{Q E}$; Fig. 7.6b) shows the CCQE (solid points) and CCQElike (empty points) purity as a function of $E_{\nu}^{Q E}$.

multiplicity requirement, while the drop at high energies is mostly due to the veto hit multiplicity and fiducial volume requirements. The CCQE purity drop with $E_{\nu}^{g e n}$ is mostly due to the increasing non-CCQE contribution to the inclusive neutrino crosssection with energy, as discussed in Chapter 5 .

Complementary information on efficiency and purity as a function of energy to the one given in Fig. 7.5 is displayed in Fig. 7.6, where the Fisher cut efficiency and the purity as a function of reconstructed neutrino energy, $E_{\nu}^{Q E}$, are shown ${ }^{1}$. The efficiency curve does not show large differences compared to the efficiency curve in terms of $E_{\nu}^{g e n}$, while the CCQE purity is expected to be less dependent on $E_{\nu}^{Q E}$ than on $E_{\nu}^{g e n}$. The reason is that a significant fraction of high-energy, non-CCQE events are expected to be interpreted, after reconstruction, as lower energy events.

\footnotetext{
${ }^{1}$ Of course, it is meaningless to define a hit-level and reconstruction-level cut efficiency in terms of reconstructed quantities, since successful reconstruction is a requirement in the reconstruction-level selection.
} 

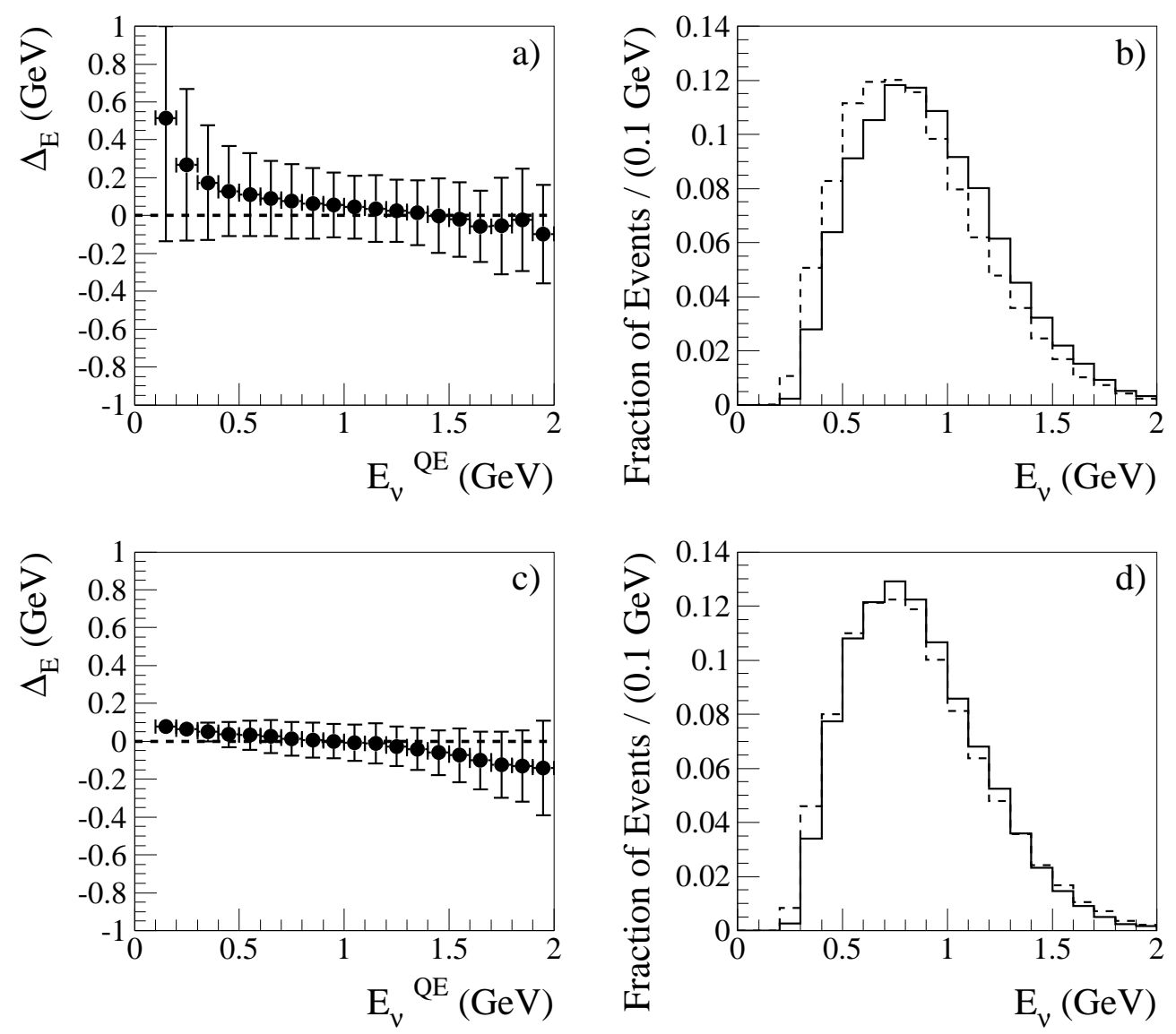

Figure 7.7: Predicted neutrino energy reconstruction performance for CCQE events. Fig. 7.7 a) shows the neutrino energy resolution $\Delta_{E}$ (see text) as a function of reconstructed neutrino energy $E_{\nu}^{Q E}$ for all events passing the CCQE selection, and Fig. 7. 7b) compares the corresponding true neutrino energy (solid line) and reconstructed neutrino energy (dashed line) distributions. Figures (7.7c) and d) are equivalent to Fig. 7.7a) and b), but for true $C C Q E$ events only passing the CCQE cuts (that is, with no non-CCQE contamination).

\subsubsection{Neutrino Energy Reconstruction}

Finally, we address the neutrino energy reconstruction performance, for CCQE candidate events in MiniBooNE. As far as the neutrino oscillation search is concerned, the better the neutrino resolution is, the more accurately the neutrino mass splitting parameter $\Delta m^{2}$ can be measured in the presence of an oscillation signal, and the stricter the constraint in the $\left(\sin ^{2} 2 \theta, \Delta m^{2}\right)$ parameter space is for the no oscillations 
case. Therefore, the neutrino energy resolution is an important figure of merit of the CCQE event selection. We define the energy bias $\overline{\Delta_{E}}$ and resolution $\delta \Delta_{E}$ as:

$$
\begin{aligned}
\overline{\Delta_{E}} & =\operatorname{Mean}\left[E_{\nu}^{g e n}-E_{\nu}^{Q E}\right] \\
\delta \Delta_{E} & =\operatorname{RMS}\left[E_{\nu}^{g e n}-E_{\nu}^{Q E}\right]
\end{aligned}
$$

The bias and resolution $\Delta_{E} \equiv \overline{\Delta_{E}} \pm \delta \Delta_{E}$ are shown in Fig. 7.7 as a function of $E_{\nu}^{Q E}$, both for all events expected to pass the CCQE selection, and ideally by retaining only the CCQE events in the sample. Figure 7.7 also shows the expected true and reconstructed neutrino energy distributions, given the predicted neutrino energy reconstruction performance. The bias and resolution are made significantly worse by the non-CCQE contribution in the sample. While the bias does not necessarily affect the $\nu_{\mu}$ disappearance sensitivity of the experiment, the resolution is expected to have an effect in the high $\Delta m^{2}$ oscillation parameter reach.

In order to quantify the overall neutrino energy reconstruction performance, we quote the fractional energy resolution averaged over the expected $E_{\nu}^{Q E}$ spectrum:

- All CCQE candidate events: $\left\langle\delta \Delta_{E}\right\rangle /\langle E\rangle=29.9 \%$

- Only CCQE events among CCQE candidate events: $\left\langle\delta \Delta_{E}\right\rangle /\langle E\rangle=11.4 \%$

\subsection{Validation of the Event Selection and Energy Reconstruction with MiniBooNE Data}

In the previous Section, we discussed the expected performance of the CCQE event selection, based on Monte Carlo simulations. In this Section we compare the event selection variables and energy distributions between simulated and real data for a variety of data sets. The purpose is to quantify the level of agreement between the actual and predicted detector response, and thereby validate the CCQE event selection and 
energy reconstruction. We show that most quantities are in good agreement between data and Monte Carlo predictions. Quantities which require further investigation are noted.

\subsubsection{Calibration Samples}

The first sample we consider is the electron calibration sample, comprising electrons from muons decaying at rest. Large differences in the output of the event selection variables are to be expected between muons from neutrino-induced CCQE interactions and Michel electrons, most importantly because of the different particles and energies involved in the two samples. Nevertheless, quantifying the agreement between data and Monte Carlo in the CCQE event selection variables and energy using Michel electrons is useful: Michel electrons test the Monte Carlo description of the detector optical model, and the performance of the reconstruction and particle ID algorithms, but do not depend on the neutrino flux and cross-section assumptions.

Figure 7.8 shows the data and Monte Carlo distributions in the reconstructed visible energy and numuCCQElike variables, for Michel electrons. Both simulated and real samples of electrons are obtained here by the decay of muons induced by neutrino interactions, with a $R_{\mu}<500 \mathrm{~cm}$ fiducial volume requirement on the decaying muon. Moreover, a tank PMT hit multiplicity requirement NTank $>50$ is imposed on the electron subevent, in order to select electrons that can be reliably reconstructed. We find reasonable agreement in the energy and numuCCQElike Fisher variable distributions, between simulated and actual Michel electrons.

Figure 7.9 shows nine of the ten event selection quantities whose linear combination forms the numuCCQElike variable, for Michel electrons (the NSubevents distribution is not shown because NSubevents $=2$ is imposed by the Michel selection). The data and Monte Carlo distributions are in agreement in most cases. One exception is given by the distribution in the BackwardHitsFraction variable, describing the 

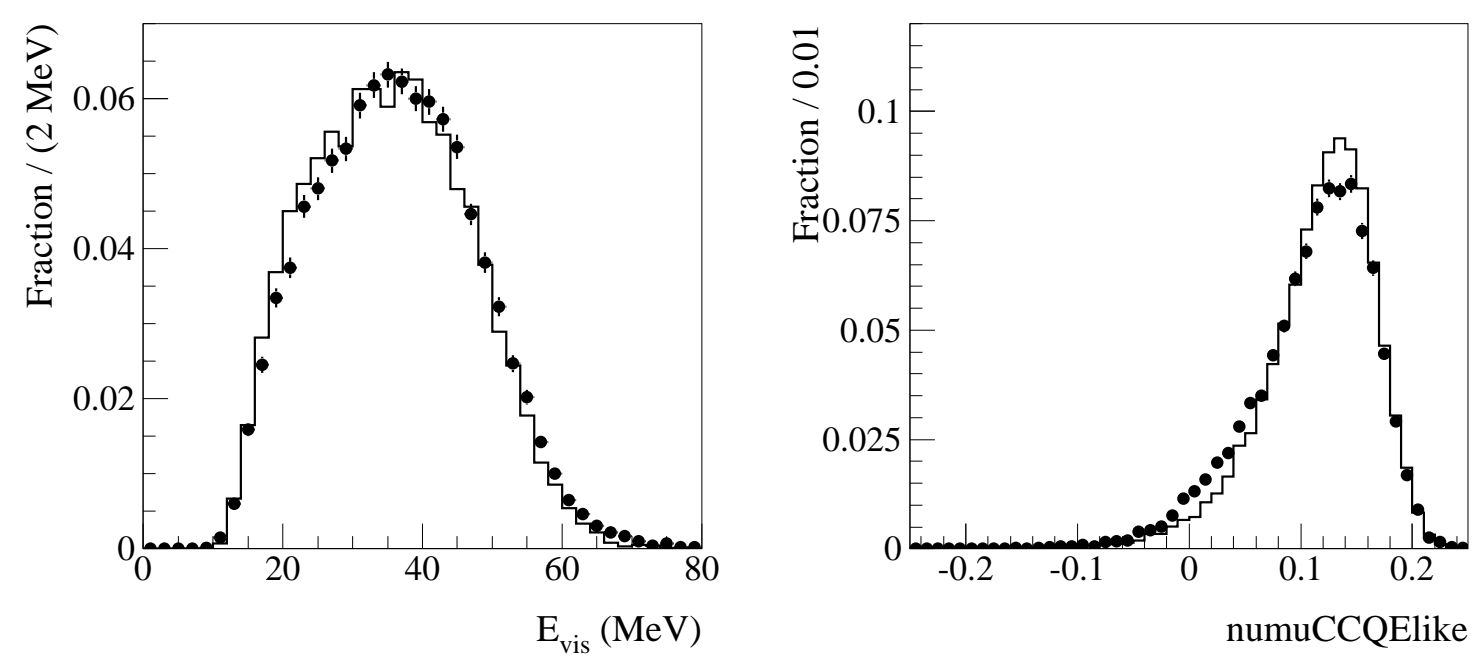

Figure 7.8: Validation of CCQE event selection using the electron calibration sample. Figure 7.8 a) shows the measured (data points) and predicted (histogram) reconstructed visible energy $E_{\text {vis }}$ in the detector. Figure $7.8 b$ ) shows the measured (data points) and predicted (histogram) CCQE Fisher variable distribution for the same sample.

fraction of prompt PMT hits that are located in the backward direction with respect to the reconstructed Cherenkov track direction.

The CCQE event selection and energy reconstruction validations have also been studied via a muon calibration sample, comprising cosmic ray muons passing through the muon tracker and stopping in the deepest scintillation cube. As discussed in Chapter 6, there are seven cubes in the MiniBooNE detector, each corresponding to a different muon range in the tank, and therefore to a different visible energy, up to about $800 \mathrm{MeV}$. The muon calibration sample studies events that have more resemblance to neutrino-induced CCQE events than the electron calibration sample events, since it consists of single muons (and not electrons) with visible energies in the range of interest for the $\nu_{\mu} \mathrm{CCQE}$ analysis. No recoil protons are present in this sample, as opposed to CCQE events. As for Michel electrons, cube muons test the Monte Carlo description of the detector optical model, and the performance of the reconstruction and particle ID algorithms, but do not depend on the neutrino flux and cross-section assumptions. The Monte Carlo sample is obtained by generating 


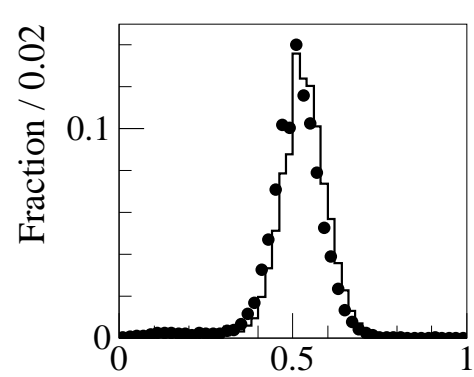

VeryPromptHitsFraction

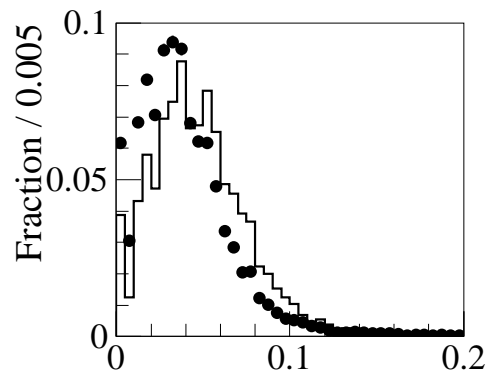

BackwardHitsFraction

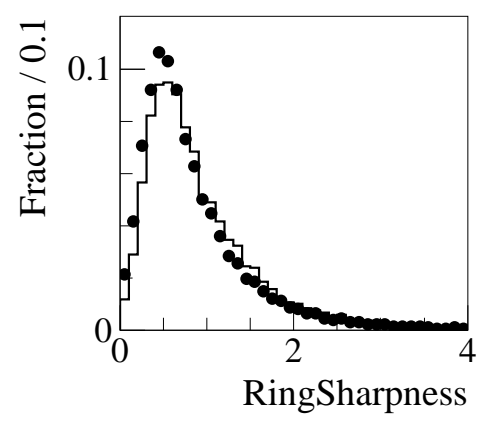

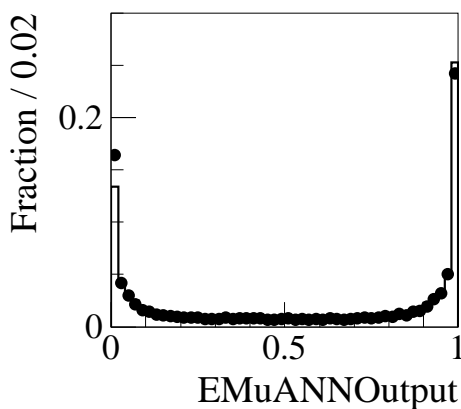

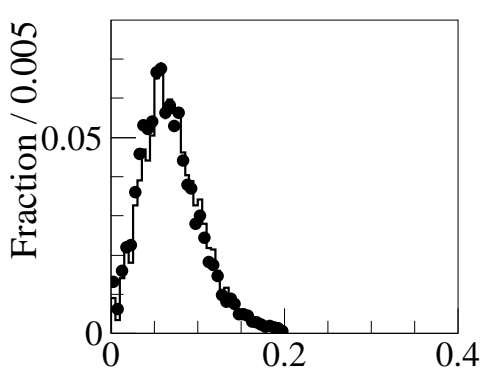

TransverseHitsFraction

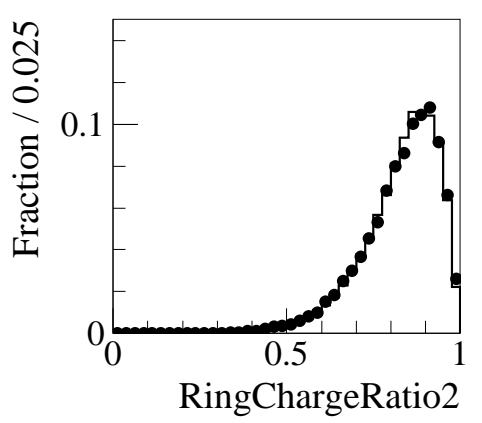

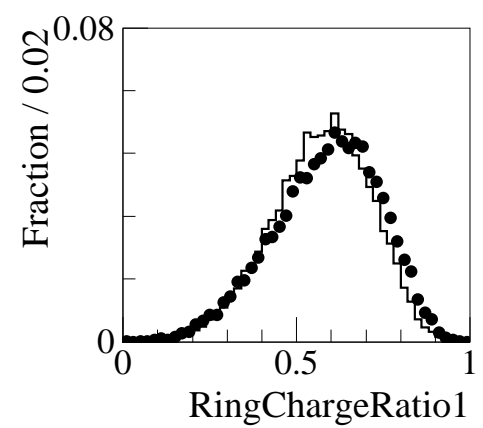
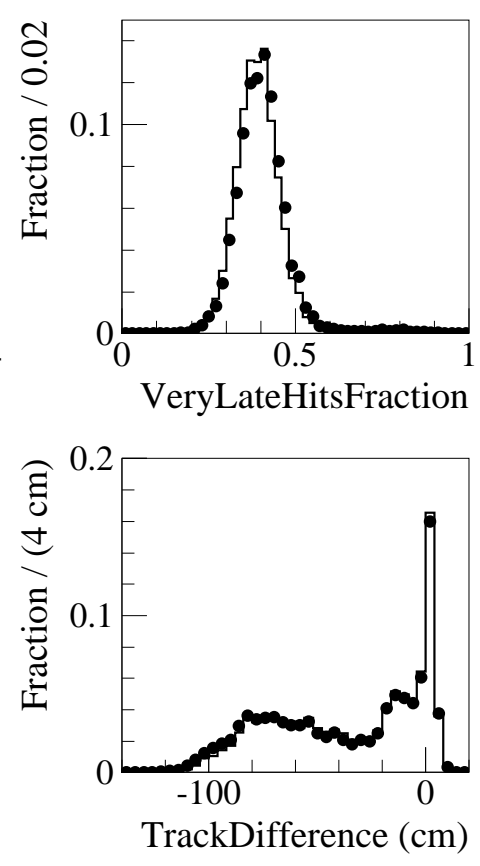

Figure 7.9: Validation of CCQE event selection using the electron calibration sample. The nine panels show the measured (data points) and predicted (histograms) distributions for the variables used as inputs to the CCQE Fisher discriminant method.

negative muons above the tracker, pointed at the cubes, with a tight range of energies so that a few percent of the muons actually hit the cubes and stop in them. For this study, only the deepest cube is used, by requiring a muon visible energy of at least $600 \mathrm{MeV}$; this requirement is imposed in order to consider muon tracks that are well within the tank fiducial volume for most of the track extent, since mostly muons of this type pass the CCQE selection cuts for neutrino-induced events.

Fig. 7.10 shows the data and Monte Carlo distributions in the visible energy $E_{v i s}$ and numuCCQElike variables, for cosmic ray muons stopping in the deepest cube. 

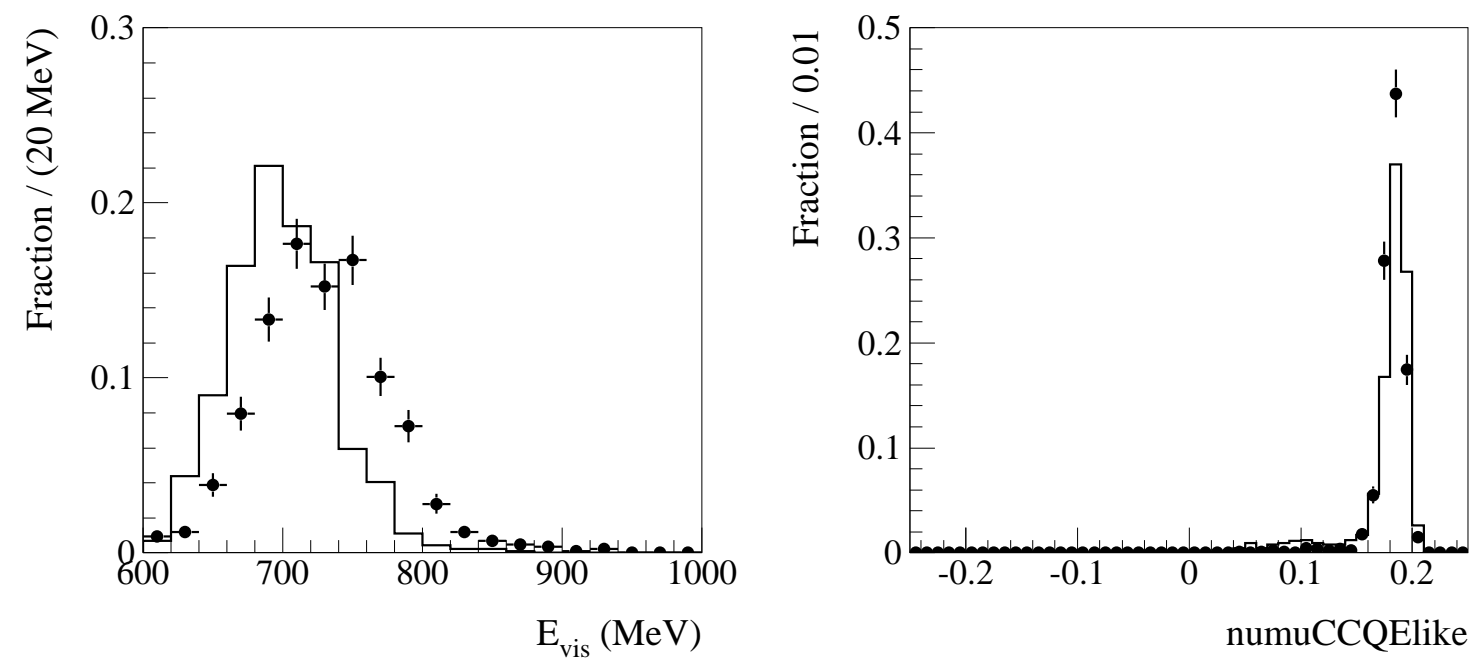

Figure 7.10: Validation of CCQE event selection using the muon calibration sample. Figure 7.10a) shows the predicted (histogram) and measured (data points) distributions for the visible energy in MiniBooNE for $E_{\text {vis }}>600 \mathrm{MeV}$, while Fig. 7.10b) shows the two CCQE Fisher variable distributions.

The agreement between data and Monte Carlo in the numuCCQElike distribution is reasonable, while the visible energy comparison is less impressive, and will need to be addressed in the future.

Fig. 7.11 shows nine of the ten event selection quantities whose linear combination forms the numuCCQElike variable, for cubes muons (the NSubevents distribution is not shown because NSubevents $=2$ is imposed by the cubes selection). Also in this case, the data and Monte Carlo distributions are in reasonable agreement.

\subsubsection{The CCQE Sample}

The third and final sample considered to validate the CCQE event selection is the CCQE candidate events sample itself. This analysis uses neutrino data collected between December, 2002, and January, 2005 (runs 3,000 to 10,493). Overall, 55,824 CCQE candidate events were collected with the event selection described above, over 


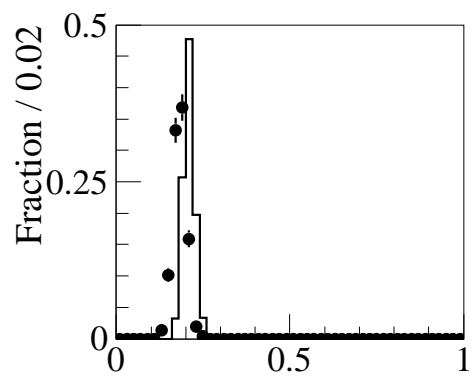

VeryPromptHitsFraction

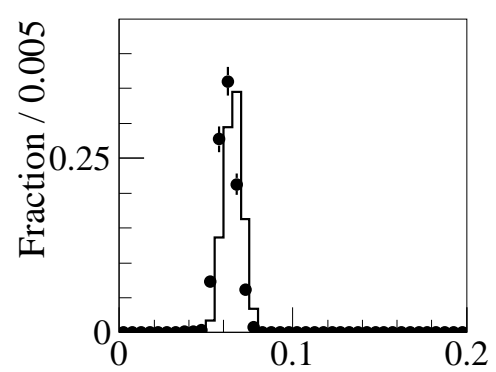

BackwardHitsFraction

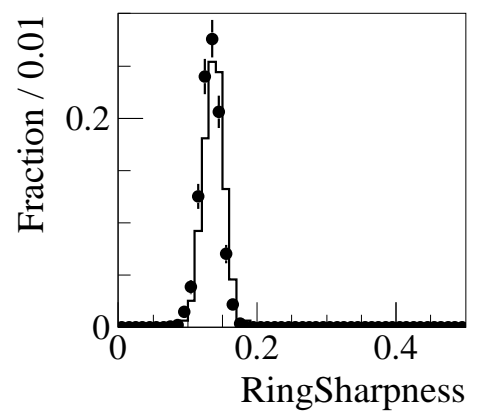

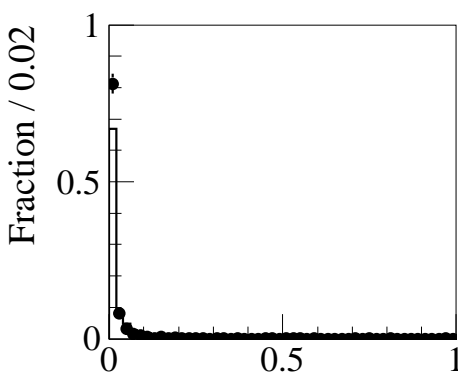

EMuANNOutput

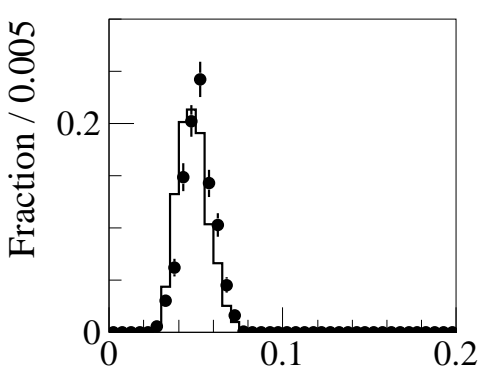

TransverseHitsFraction

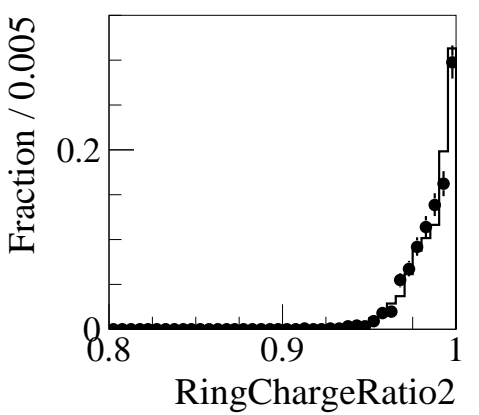

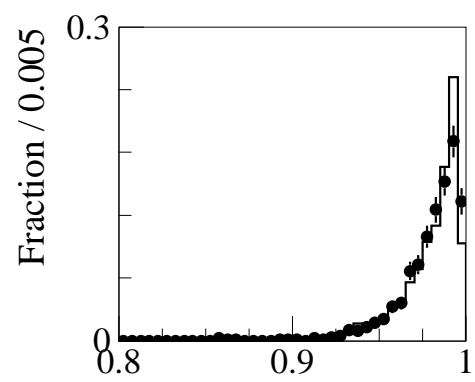

RingChargeRatio1

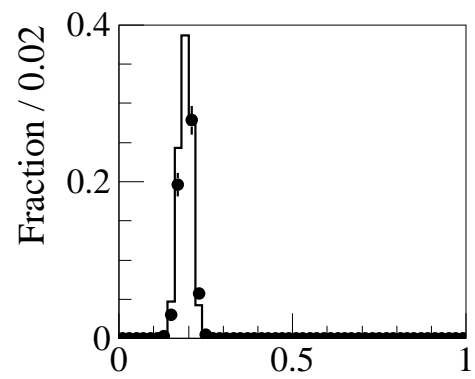

VeryLateHitsFraction

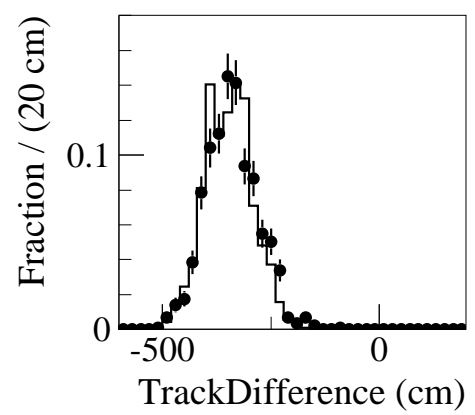

Figure 7.11: Validation of CCQE event selection using the muon calibration sample. The nine panels show the measured (data points) and predicted (histograms) distributions for the variables used as inputs to the CCQE Fisher discriminant method.

this time period 2 , In addition to the hit-level, reconstruction-level, and event-level cuts, other data-quality criteria are applied: the detector and beam datastreams are both required to exist and to be successfully merged in time, detector data is required to be not latent, and the MiniBooNE horn is required to have pulsed at full current, in coincidence with the proton beam spill on target.

The ten Fisher input variables are displayed in Fig. 7.12, while Fig. 7.13 shows

\footnotetext{
${ }^{2}$ This datastream has a prescale factor of 2 , so that twice as many CCQE candidate events would be present without prescaling.
} 

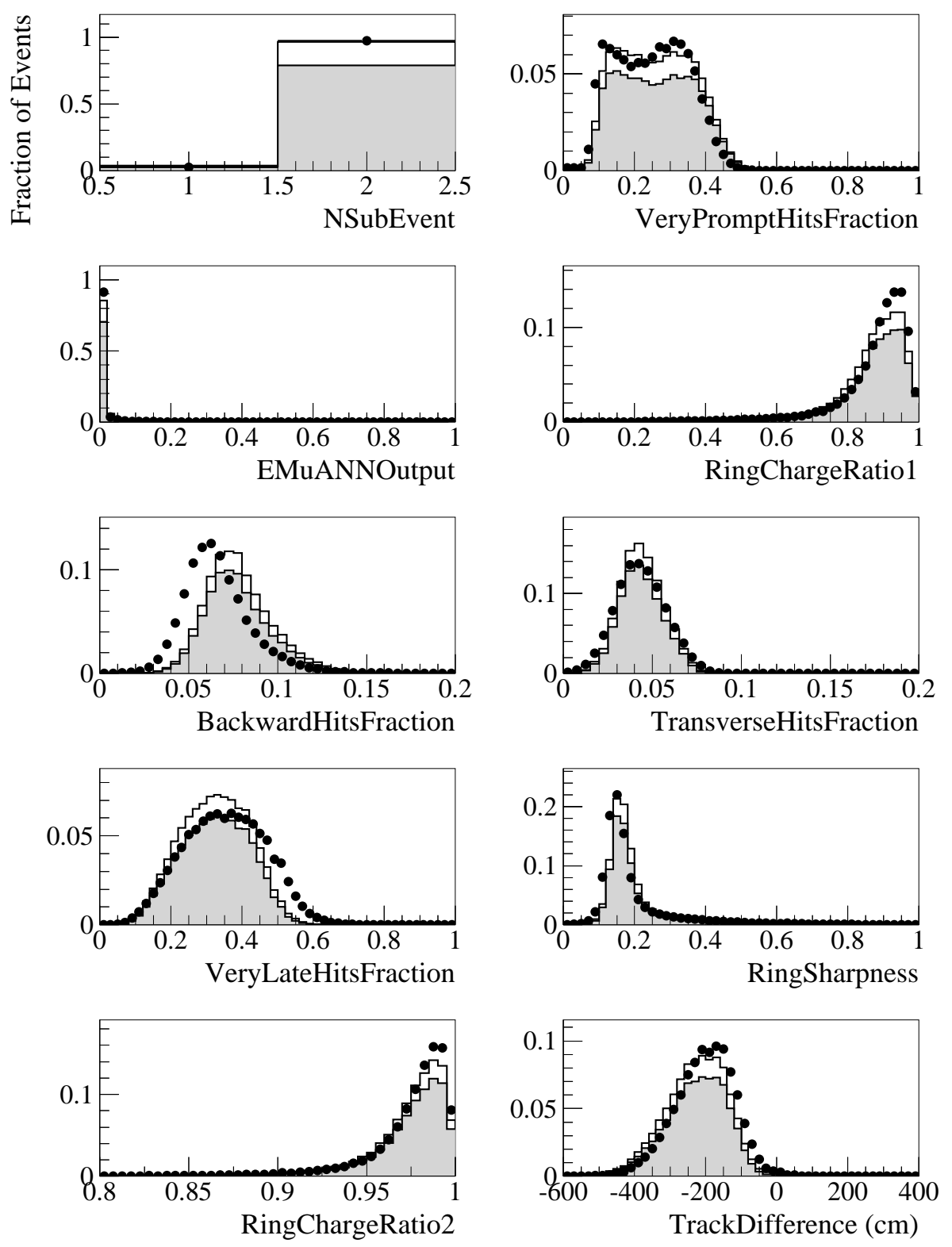

Figure 7.12: Validation of CCQE event selection using CCQE candidate events in MiniBooNE (numuCCQElike>0.14). The ten panels show the measured (data points) and predicted (histograms) distributions for the variables used as inputs to the CCQE Fisher discriminant method. The filled histograms show the expected CCQE contributions.

the data and Monte Carlo numuCCQElike distributions. The filled histograms in Figs. 7.12 and 7.13 show the expected contributions from CCQE events in the sample. In general, the level of agreement is satisfactory, with the exception of 


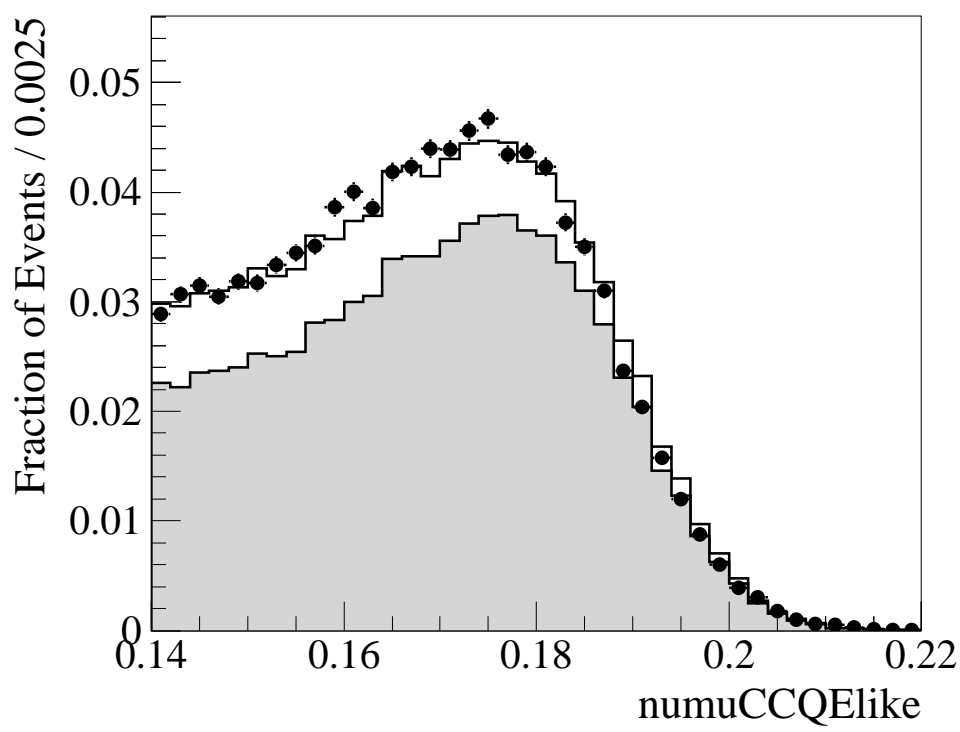

Figure 7.13: Validation of CCQE event selection using CCQE candidate events in MiniBooNE. The Figure shows the measured (data points) and predicted (histogram) CCQE Fisher variable distribution for CCQE candidate events (numuCCQElike>0.14). The filled histogram shows the expected CCQE contribution.

the BackwardHitsFraction and VeryLateHitsFraction distributions. Despite this, however, the predicted PID variable numuCCQElike, used to select CCQE candidate events and shown in Fig. 7.13, match quite well the measured one.

\subsection{CCQE Results}

Having discussed the CCQE event selection description, performance, and validation, we can now make preliminary comparisons between the measured and predicted MiniBooNE $\nu_{\mu}$ CCQE event distributions of physics interest, based on the same data sample described in Section 7.3.2. We proceed in increasing order of complexity, by displaying hit-level distributions first, then showing distributions related to the properties of the final state muon, of the initial state neutrino, and of the full CCQE interaction kinematics. All distributions shown are relatively normalized. 

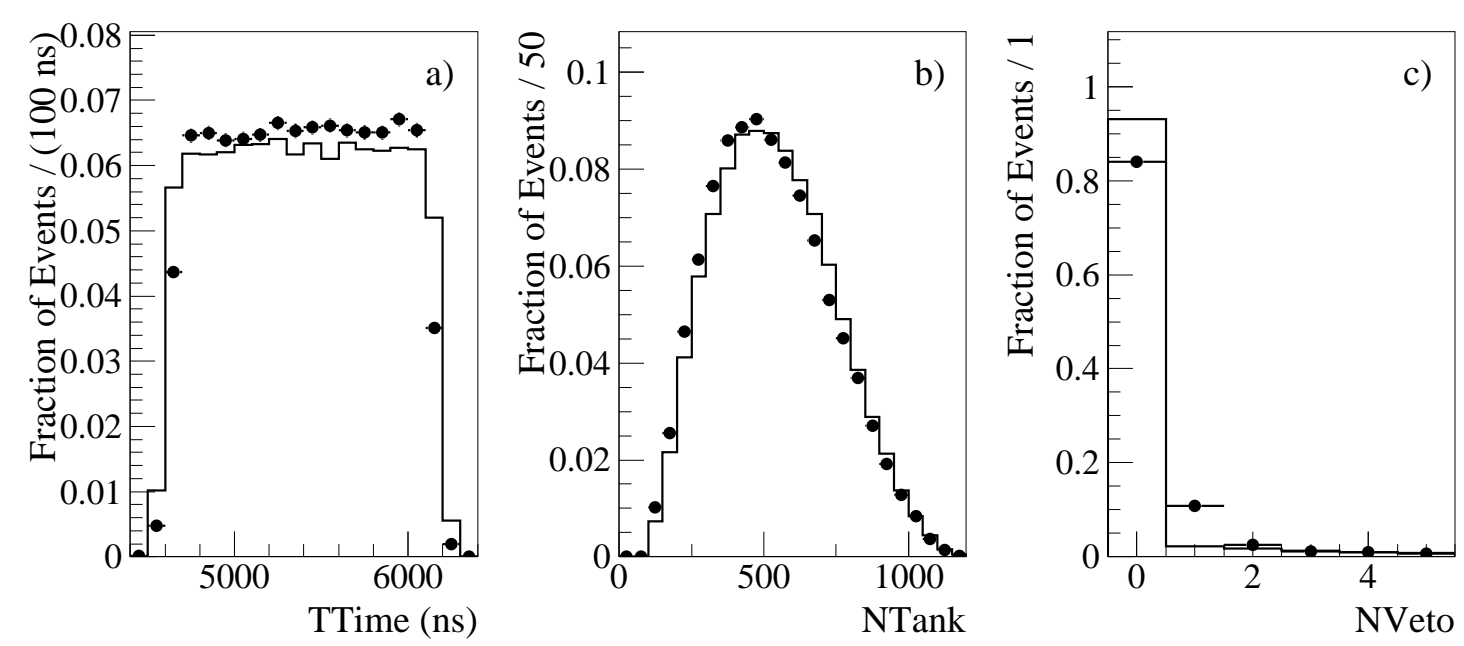

Figure 7.14: Comparison between measured (data points) and predicted (histograms) hitlevel distributions for CCQE candidate events. Figure 7.14a) shows the average sub-event time distribution, Fig. 7.14 b) the main tank PMT hit multiplicity, Fig. 7.14c) the veto PMT hit multiplicity.

Figure 7.14 shows data and Monte Carlo hit-level distributions for the first subevent: average subevent time TTime, main tank hit multiplicity NTank, and veto hit multiplicity NVeto. The hit multiplicities, roughly proportional to the visible energy in the detector, match reasonably well, with about half of the 1280 main PMTs hit by light, and no veto activity, on average. The measured TTime distribution shows the characteristic beam spill duration of $1.6 \mu \mathrm{s}$ of the Booster extraction.

Figure 7.15 shows data and Monte Carlo distributions related to the reconstructed properties of the final state muon: muon kinetic energy $T_{\mu}$, cosine of the muon direction with respect to the neutrino direction $\cos \theta_{\mu}$, and radial position of the mean light emission point of the muon track in equal-volume shells, $\left(R_{\mu} / 500 \mathrm{~cm}\right)^{3}$. The mean light emission point roughly corresponds to the mid-point along the muon track. In MiniBooNE CCQE interactions, muons tend to be produced along the incoming neutrino direction, and with a kinetic energy of about $500 \mathrm{MeV}$. Moreover the event selection criteria cause a marked departure from a uniform, flat in $R_{\mu}^{3}$, muon spatial distribution in the tank; this departure is well-modeled by the Monte Carlo 

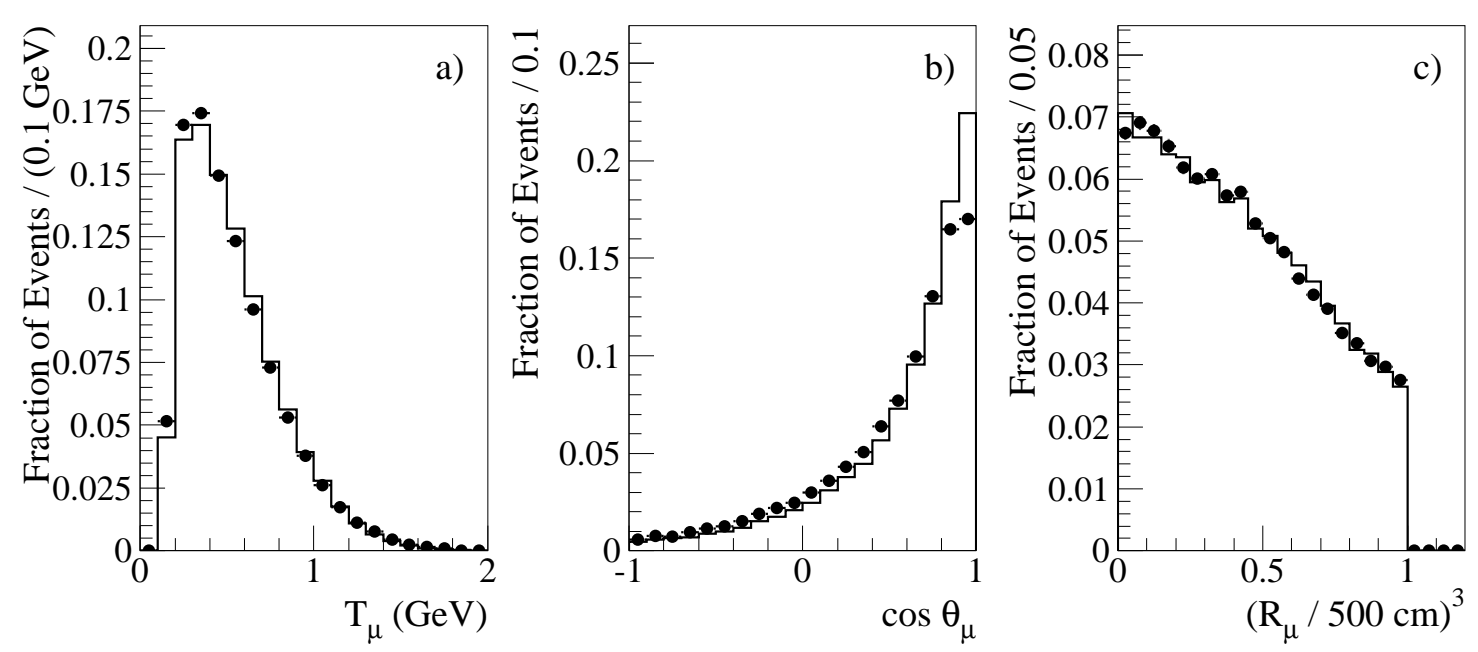

Figure 7.15: Comparison between measured (data points) and predicted (histograms) distributions related to the final-state muon in CCQE candidate events. Figure 7.15a) shows the reconstructed muon kinetic energy, Fig. 7.15b) the reconstructed muon track direction with respect to the neutrino direction, Fig. 7.15 c) the reconstructed, equal-volume radial position distribution of the mean light emission point along the muon track.

simulation, as is the energy scale of the detector as given by $T_{\mu}$. On the other hand, the muon angular distribution appears flatter than what the neutrino event generator and the Monte Carlo simulation of the detector predict. The dicrepancy between real and simulated data in the $\cos \theta_{\mu}$ distribution near $\cos \theta_{\mu} \simeq 1$ is discussed below, in conjunction with the related $Q^{2}$ distribution comparison.

Figure 7.16 shows the observed and predicted distributions in the reconstructed neutrino energy $E_{\nu}^{Q E}$ and in the radial position of the neutrino interaction vertex in equal-volume shells, $\left(R_{\nu} / 500 \mathrm{~cm}\right)^{3}$. Charged-current, quasi-elastic neutrino interactions in MiniBooNE are characterized by typical energies of about $900 \mathrm{MeV}$. The neutrino interaction vertex distribution is reasonably well modelled by the Monte Carlo simulation, and shows a marked departure from uniformity due to the event selection criteria, as for the corresponding muon distributions. The predicted reconstructed neutrino energy distribution, assuming no oscillations, matches MiniBooNE observations reasonably well. The neutrino energy comparison is discussed further in 

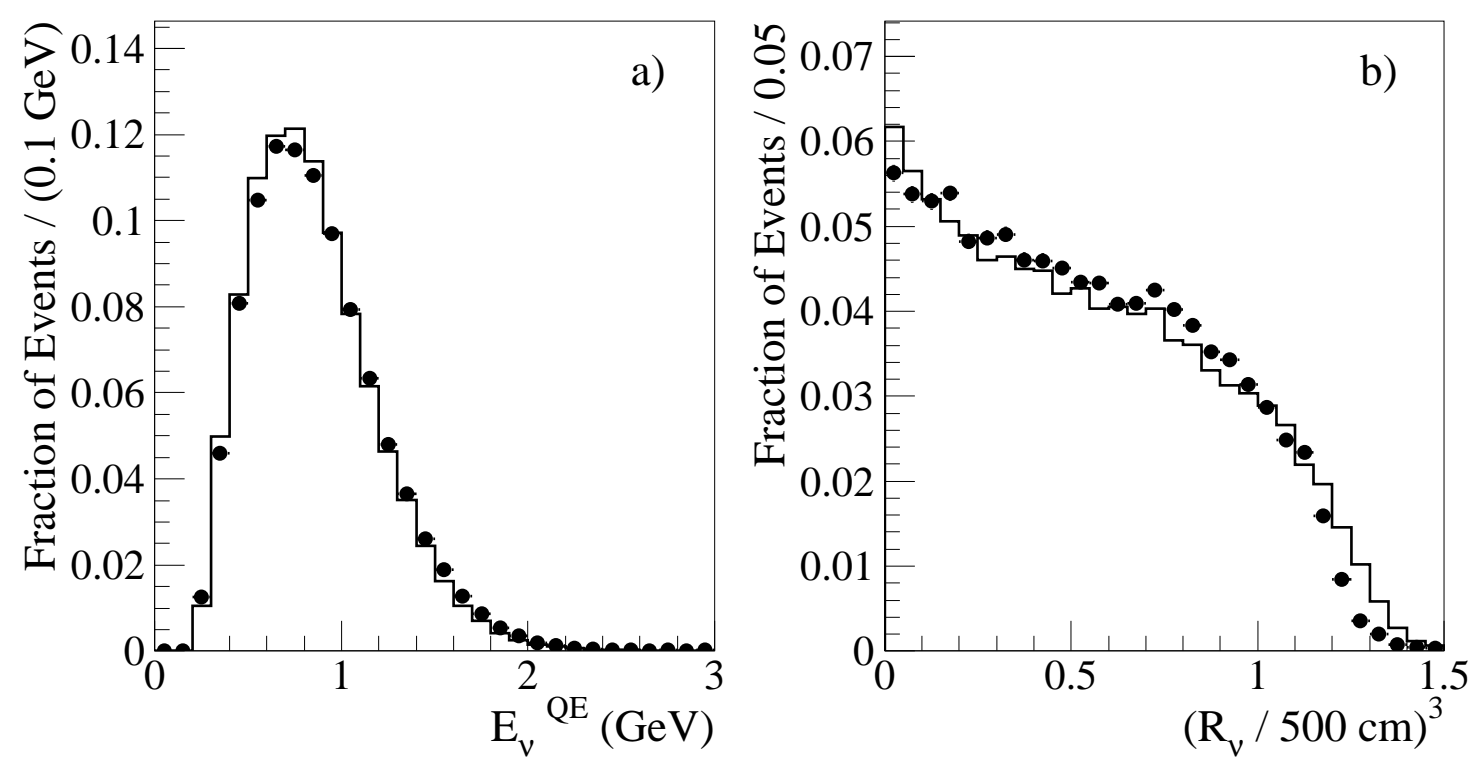

Figure 7.16: Comparison between measured (data points) and predicted (histograms) distributions related to the initial-state neutrino in CCQE candidate events. Figure 7.16a) shows the reconstructed neutrino energy, Fig. 7.16 b) shows the reconstructed, equal-volume radial position distribution of the neutrino interaction vertex..

Chapter 8, focusing on the muon neutrino disappearance analysis.

Finally, Fig. 7.17 compares observed and predicted distributions related to the full event kinematics in MiniBooNE CCQE candidate events: $Q^{2}$ of the fourmomentum transfer between the neutrino and the muon given by Eq. 7.7, the "inelasticity" $\nu \equiv E_{\nu}^{Q E}-\left(T_{\mu}+m_{\mu}\right)$ of the interaction, as well as the $Q^{2}$ versus $\nu$ scatter-plot. MiniBooNE CCQE interactions probe mostly the low $Q^{2}$ (less than about $1 \mathrm{GeV}^{2}$ ), low $\nu$ (less than about $0.5 \mathrm{GeV}$ ) region. The observed and predicted $Q^{2}$ versus $\nu$ relationships are in fairly good agreement with each other for all $\nu$ values, and in reasonable agreement with the relationship $Q^{2}=2 M_{N} \nu$, which is expected for CCQE interactions that are not affected by reconstruction, nuclear Fermi momentum, or binding energy effects, as discussed in Chapter 5. The observed deviations from $Q^{2}=2 M_{N} \nu$ are well modeled by the Monte Carlo simulation.

In contrast, and as for the related forward part of the muon angular distribu- 

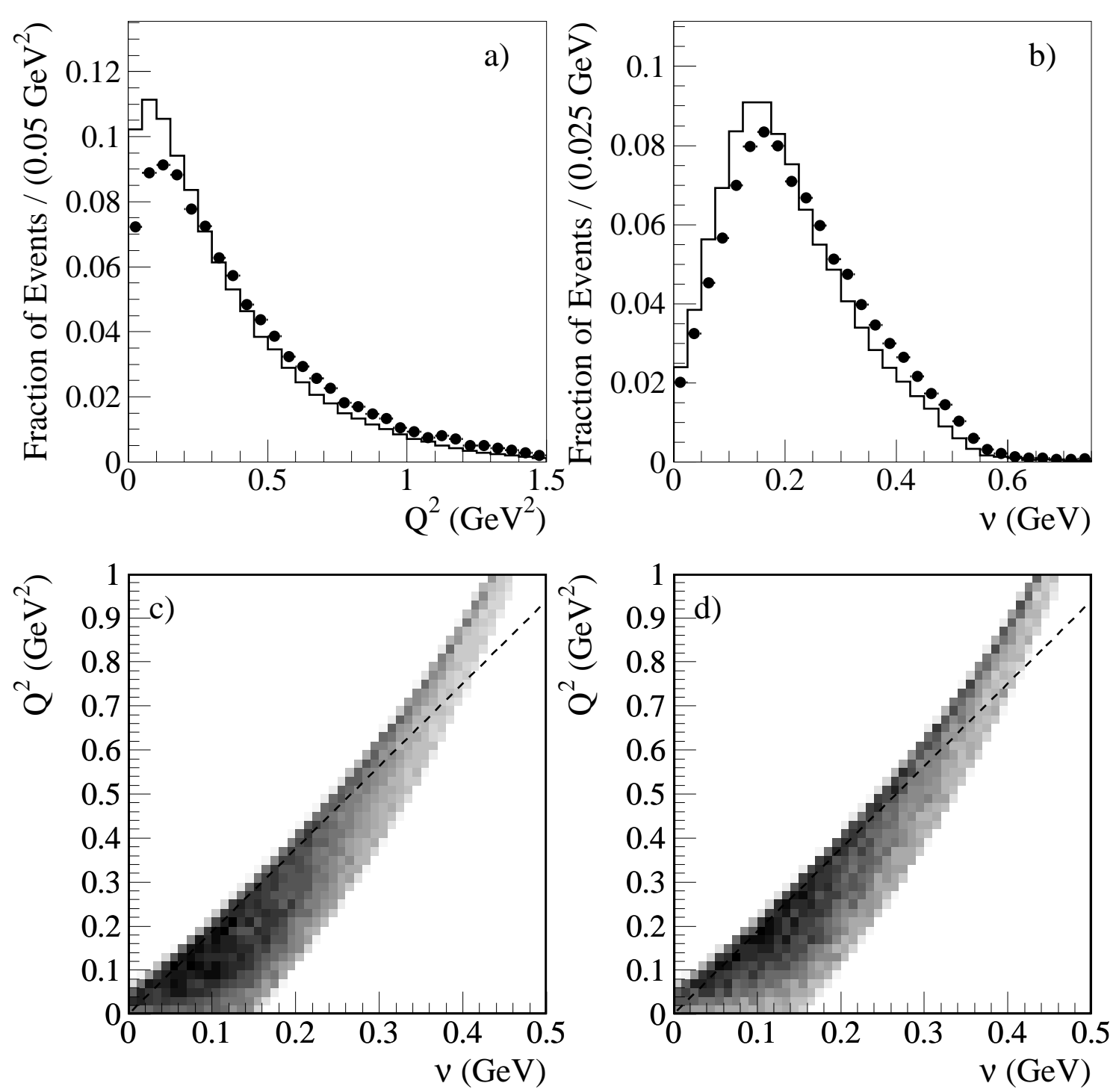

Figure 7.17: Comparison between measured (data points) and predicted (histograms) distributions related to the full-event kinematics in CCQE candidate events. Figure 7.17a) shows the reconstructed $Q^{2}$ distributions, Fig. 7.17) the reconstructed $\nu$ distributions, Figs. $7.17_{c}$ ) and d) the predicted and measured $Q^{2}$ versus $\nu$ scatter plots, respectively. The dashed line in Figs. $7.17 c$ ) and d) indicates the $Q^{2}=2 M_{N} \nu$ expectation.

tion $\cos \theta_{\mu}$ in Fig. 7.15, the data appears to show a rate-deficit in the lowest $Q^{2}$ bins, below about $0.15 \mathrm{GeV}^{2}$, while the data and Monte Carlo $\nu$ distributions are in better (but not perfect) agreement. The low $Q^{2}$ region is expected to probe nuclear effects in neutrino interactions, and the discrepancy between the observations and 
predictions may point to unsimulated physics in the description of neutrino-nucleus cross-sections, even though no definitive statement can be made at this preliminary stage. A similar low $Q^{2}$ rate deficit in neutrino cross-section rates on a Carbon nuclear target has been seen by the K2K experiment in their non-CCQE enriched neutrino samples [149]. 


\section{Chapter 8}

\section{The Muon Neutrino Disappearance}

\section{Analysis}

The goal of the MiniBooNE $\nu_{\mu}$ disappearance analysis is to constrain neutrino oscillations of muon-type neutrinos into neutrinos that cannot be observed in the MiniBooNE detector, such as sterile or tau neutrinos. This search is motivated in Chapter 2. where the possibility for large $\nu_{\mu} \rightarrow \nu_{s}$ oscillation probabilities in the neutrino mass splitting range accessible to MiniBooNE is substantiated. Muon neutrino disappearance can be constrained in MiniBooNE by comparing the overall number of observed and predicted $\nu_{\mu}$ interactions in the detector (normalization-only analysis), by looking for neutrino energy-dependent distortions in the observed distributions compared to the predicted ones (shape-only analysis), or by combining both comparisons (normalization+shape analysis). In this Chapter, the MiniBooNE sensitivity to muon neutrino disappearance via a shape-only analysis is discussed.

The aim of this Chapter is to piece the findings of the previous chapters together, in order to study the MiniBooNE sensitivity to neutrino masses and mixings via muon neutrino disappearance. 


\subsection{The Oscillation Fitting Code}

The goal of the oscillation fitting code is to determine the goodness-of-fit of the oscillation and no-oscillation hypotheses, as well as computing the estimation (bestfit and errors) of the neutrino oscillation parameters $\left(\sin ^{2} 2 \theta_{\mu \mu}, \Delta m^{2}\right)$. The oscillation fits discussed here assume that only one neutrino mass splitting may contribute to the possible disappearance of MiniBooNE muon neutrinos; this assumption is valid, for example, for the $(3+1)$ sterile neutrino models discussed in Chapter 2 .

Observations and Monte Carlo predictions for the yields of CCQE candidates as a function of neutrino energy are compared using the following $\chi^{2}$ definition:

$$
\chi^{2}\left(\Delta m^{2}, \sin ^{2} 2 \theta_{\mu \mu}, k\right)=\sum_{\alpha, \beta}\left(N_{\alpha}^{o b s}-k N_{\alpha}^{p r e d}\right)\left(M^{-1}\right)_{\alpha \beta}\left(N_{\beta}^{o b s}-k N_{\beta}^{p r e d}\right)
$$

where $N_{\alpha}^{\text {pred }}=N_{\alpha}^{\text {pred }}\left(\Delta m^{2}, \sin ^{2} 2 \theta_{\mu \mu}\right)$ is the predicted yield in the reconstructed neutrino energy bin $\alpha, N_{\alpha}^{o b s}$ is the observed yield, $M_{\alpha \beta}$ is the error matrix in bins of reconstructed neutrino energy, $k$ is an observation-to-prediction yield ratio parameter. For energy shape-only fits as the ones discussed in this Chapter, the $\chi^{2}$ function of Eq. 8.1 is minimized by varying the three fit parameters $\left(\Delta m^{2}, \sin ^{2} 2 \theta_{\mu \mu}, k\right)$, and no normalization information is used.

For a better understanding of the construction of the error matrix appearing in Eq. 8.1, and discussed in the next Section, it is useful to explicitly discuss how the predicted yields $N_{\alpha}^{\text {pred }}$ are obtained. The number of predicted events can be expressed as:

$$
N_{\alpha}^{\text {pred }}=\sum_{i} N_{\mathrm{pot}} \cdot N_{\mathrm{CH}_{2}} \cdot \phi_{i}\left(\frac{1}{\mathrm{~cm}^{2} \cdot \mathrm{pot}}\right) \cdot \sigma_{i}\left(\frac{\mathrm{cm}^{2}}{\mathrm{~N}_{\mathrm{CH}_{2}}}\right) \cdot \epsilon_{\alpha i} \cdot\left\langle p_{\nu_{\mu} \rightarrow \nu_{\iota}}\left(\sin ^{2} 2 \theta_{\mu \mu}, \Delta m^{2}\right)\right\rangle_{i}
$$

where $N_{\text {pot }}$ is the number of protons reaching the MiniBooNE beryllium target, $\mathrm{N}_{\mathrm{CH}_{2}}$ is the number of $\mathrm{CH}_{2}$ molecules in the MiniBooNE detector ${ }^{1}, \phi_{i}\left(\frac{1}{\mathrm{~cm}^{2} \cdot \mathrm{pot}}\right)$ is the flux per unit area and per proton on target in the true neutrino energy bin $i$,

\footnotetext{
${ }^{1}$ The MiniBooNE detector is filled with mineral oil, which can be approximated as $\mathrm{CH}_{2}$ molecules.
} 
$\sigma_{i}\left(\frac{\mathrm{cm}^{2}}{\mathrm{~N}_{\mathrm{CH}_{2}}}\right)$ is the neutrino cross-section per $\mathrm{CH}_{2}$ molecule for a neutrino of energy $E_{i}$, $\epsilon_{\alpha i}$ is the probability of measuring a neutrino interaction with reconstructed energy in bin $\alpha$ for a neutrino of true energy $E_{i}$ interacting within the MiniBooNE detector, given the CCQE selection described in Chapter 6, and $\left\langle p_{\nu_{\mu} \rightarrow \nu_{\mu}}\left(\sin ^{2} 2 \theta_{\mu \mu}, \Delta m^{2}\right)\right\rangle_{i}$ is the $\nu_{\mu}$ disappearance probability averaged over the true neutrino energy bin $i$. The $\epsilon_{\alpha i}$ term can further be expressed as the product of the CCQE selection efficiency parametrized in terms of true neutrino energy, times a migration matrix relating true and reconstructed neutrino energies. The smearing in the distance between neutrino production and neutrino detection, mostly due to the $50 \mathrm{~m}$ long decay region, is taken into account in the oscillation probability term. The muon neutrino survival probability term is equal to one for the no-oscillation prediction, and the predicted yield is indicated by $N_{\alpha, 0}^{\text {pred }} \equiv N_{\alpha}^{\text {pred }}\left(\sin ^{2} 2 \theta_{\mu \mu}=0\right)$ in the following. Uncertainties in $N_{\text {pot }}$ and $N_{\mathrm{CH}_{2}}$ would enter in the analysis as normalization-only uncertainties, and need not be taken into account for a shape-only analysis. Moreover, we assume that $\left\langle p_{\nu_{\mu} \rightarrow \nu_{\mu}}\left(\sin ^{2} 2 \theta_{\mu \mu}, \Delta m^{2}\right)\right\rangle_{i}$ is known with negligible errors, since the uncertainties related to the simulation of the neutrino baseline smearing, and to the evaluation of the oscillation integral over the neutrino energy bin, are small. The remaining three multiplicative terms in Eq. 8.2 are separately related to flux, cross-section, and detector response predictions. The systematic uncertainties affecting those predictions are discussed in the next Section.

\subsection{Systematic Errors Assumptions}

The error matrix $M_{\alpha \beta}$ appearing in Eq. 8.1 can be broken up into four independent error matrices: the one associated with flux systematic uncertainties $\left(M_{\alpha \beta}^{\mathrm{flux}}\right)$, neutrino cross-section uncertainties $\left(M_{\alpha \beta}^{\mathrm{xsec}}\right)$, detection uncertainties $\left(M_{\alpha \beta}^{\mathrm{det}}\right)$, and statistical uncertainties $\left(M_{\alpha \beta}^{\text {stat }}\right)$. The four error matrices are summed to provide the final error 
matrix appearing in the $\chi^{2}$ function defined in Eq. 8.1.

$$
M_{\alpha \beta}=M_{\alpha \beta}^{\mathrm{flux}}+M_{\alpha \beta}^{\mathrm{xsec}}+M_{\alpha \beta}^{\mathrm{det}}+M_{\alpha \beta}^{\mathrm{stat}}
$$

The statistical error matrix is straightforward to calculate given the observed yields:

$$
M_{\alpha \beta}^{\text {stat }}=\sqrt{N_{\alpha}^{\text {obs }}} \delta_{\alpha \beta}
$$

The statistical error matrix is diagonal since the statistical error in a certain reconstructed energy bin $\alpha$ is uncorrelated with the statistical error in a different bin $\beta$.

The general formalism used for constructing the systematic error matrices associated with flux, cross-section, and detection uncertainties is first presented. The construction of these three error matrices is of particular importance, since the large statistical sample of MiniBooNE CCQE candidate events already collected in MiniBooNE makes the $\nu_{\mu}$ disappearance search a systematics-limited measurement. The parameters describing flux, cross-section, and detection systematic uncertainties are propagated into an uncertainty related to the MiniBooNE CCQE sample via the following first-order approximation in a given systematic source variation $S_{i}$ :

$$
M_{\alpha \beta}=\overline{N_{\alpha, 0}^{\text {pred }}} \overline{N_{\beta, 0}^{\text {pred }}} D_{\alpha i} M_{i j}^{\prime} D_{\beta j}^{T}
$$

where $\alpha$ and $\beta$ label bins in reconstructed neutrino energy, $i$ and $j$ now label a systematic parameter that describes a systematic uncertainty associated with either flux, cross-section, or detector response uncertainties, $M_{i j}^{\prime}$ is the input error matrix in the systematic parameters $S_{i}, D_{\alpha i}$ is a matrix of first-derivatives that propagate the uncertainty in the systematic parameter $S_{i}$ into an uncertainty on CCQE yields in bin $\alpha$. Generally, the propagation matrix $D$ is computed via Monte Carlo simulations, while the accuracy with which a systematic parameter $S_{i}$ is known is encoded in $M_{i j}^{\prime}$.

For energy shape-only fits, the propagation matrix $D$ is constructed from the fractions of events in a neutrino energy bin $\alpha$ obtained with and without varying the systematic parameter $S_{i}$ by some small amount $\delta S_{i}$ :

$$
D_{\alpha i}=\frac{1}{\bar{n}_{\alpha}} \frac{\partial n_{\alpha}}{\partial S_{i}} \simeq \frac{1}{\bar{n}_{\alpha}} \frac{n\left(\bar{S}_{i}+\delta S_{i}\right)_{\alpha}-\bar{n}_{\alpha}}{\delta S_{i}}
$$


where $n_{\alpha} \equiv N_{\alpha}^{\text {pred }} / N^{\text {pred }}$ is the fraction of CCQE candidate events predicted for no oscillations in the energy bin $\alpha\left(e . g . \sum_{\alpha} n_{\alpha} \equiv 1\right)$.

Error matrices constructed in this way fully take into account correlations among different energy bins. Implicit in the method described above to evaluate systematic uncertainties are the following four assumptions: (1) the flux, cross-section, and detection uncertainties are independent, and therefore uncorrelated; (2) the first-order approximation used for propagating uncertainties holds approximately true given the size of the systematic uncertainties $\delta S_{i}$; (3) the propagation matrix $D$ is calculated with sufficient Monte Carlo statistics compared to the systematic variation: $\delta n_{\alpha} / \bar{n}_{\alpha} \ll\left(N_{\alpha, 0}^{\text {pred }}\right)^{-1 / 2} ;$ (4) the uncertainties in the systematic parameters $S_{i}$ are gaussian-distributed.

Given this formalism, we now summarize the systematic uncertainties considered in the analysis, and how they affect the predicted energy shape of the MiniBooNE CCQE sample.

\subsubsection{Neutrino Flux}

As mentioned in Chapter 4, we consider four sources of systematic uncertainties affecting the muon neutrino flux predictions: uncertainties in the double-differential, inclusive $\pi^{+}$and $K^{+}$production cross-sections in proton-beryllium interactions, in the proton-beryllium total inelastic cross-section, and in the description of hadronic interactions affecting the propagation of secondary particles produced in the MiniBooNE target. The double-differential, inclusive $\pi^{+}\left(K^{+}\right)$production cross-sections are parametrized in terms of eight (nine) Sanford-Wang parameters $S_{i}$. The error matrix $M_{i j}^{\prime}$ in Eq. 8.5. describing how well these parameters are known, is obtained from the combined fit to existing pion and kaon production data in the beam momentum and pion phase space of relevance to MiniBooNE, as discussed in Chapter 4 From those same fits, a $7 \%$ uncertainty in the total proton-beryllium inelastic 
cross-section is estimated. Finally, the uncertainty due to the description of hadronic interactions of secondary particles produced in the target is estimated by varying the physics model used to describe those processes in the beam Monte Carlo simulation. The flux uncertainties given above are preliminary, and will be revised when HARP data will become available.

The uncertainty in the CCQE yields due to the flux uncertainty is obtained in two steps: first, an error matrix in the fractional flux prediction as a function of generated neutrino energy is obtained from the beam Monte Carlo simulation; second, this flux prediction uncertainty is propagated into an uncertainty in the CCQE candidate yields as a function of reconstructed neutrino energy by using the MiniBooNE NUANCE event generator, the detector Monte Carlo simulation, and the reconstruction

software. This two-step approach is a good approximation to Eq. 8.5, and takes into account correlations among different energy bins, and the energy dependence of the neutrino cross-section and of the CCQE selection efficiency, given the similarity between generated and reconstructed neutrino energies, and given the sufficiently large number of energy bins chosen (typically 30 bins between 0 and $3 \mathrm{GeV}$ ).

\subsubsection{Neutrino Cross-sections}

The effect on the predicted CCQE yields due to several sources of neutrino crosssection uncertainties has been studied. Details on the neutrino cross-section parameters listed below are given in Chapter 5. Uncertainties associated with the physics model responsible for the nuclear effects in neutrino cross-sections are accounted for by assuming a $25 \mathrm{MeV}$ uncertainty in the binding energy, and a $30 \mathrm{MeV} / \mathrm{c}$ uncertainty in the Fermi momentum parametrizing the Fermi gas model. Form factor uncertainties are considered by assigning a 10\%,20\%, and $35 \%$ uncertainty in the axial mass describing quasi-elastic, single- $\pi$ production, and multi- $\pi$ production crosssections, respectively. A $100 \%$ uncertainty is assigned to the coherent $\pi$ production 
contribution. Uncertainties in the final state interaction effects are accounted for by assuming a $25 \%$ and $30 \%$ uncertainty in the $\pi$ absorption and charge-exchange crosssections, respectively. Additional uncertainties in neutrino interactions proceeding via $\Delta$-resonances are estimated by assigning a $50 \%$ uncertainty to the $\Delta N \rightarrow N N$ rate, and a $4 \%$ uncertainty to the $\Delta$-resonance width.

The cross-section uncertainties listed above change not only the overall neutrino interaction rate prediction in the detector as a function of energy, but also the final state of the neutrino interactions, therefore affecting the predicted behavior of the reconstruction and of the CCQE event selection. For this reason, events generated according to the assigned cross-section uncertainties are fully propagated through the MiniBooNE analysis chain.

\subsubsection{Detector Response}

Uncertainties in the predicted neutrino energy reconstruction, and in the efficiency and purity with which CCQE events are selected, depend upon how well the detector response is simulated. A full account of the uncertainties associated with the detector response is not currently available, and it is a subject of intense study within the experiment. As mentioned in Chapter 6, several processes are expected to affect light production and propagation in the detector, and the task of assigning uncertainties to the description of those processes in the detector simulation is not trivial.

In order to partially address this currently unknown, but potentially significant, systematic effect on the CCQE sample predictions, the cosmic ray muon calibration sample discussed in Chapter 7 is used, in order to estimate the neutrino energy reconstruction systematic uncertainty. The uncertainty described below is preliminary, and will be revised when full estimates will become available. Moreover, no attempt has been made here to estimate systematic effects on the CCQE event selection due to the detector optical model uncertainties. 
The energy deposited in the detector by cosmic ray muons traversing the muon tracker system, and stopping inside a scintillation cube in the detector, can be estimated very accurately, given the known pathlength and energy deposition characteristics of muons. For these muons of known energy, we study the potential differences in the reconstructed muon energy in data and in Monte Carlo simulations, while the overall absolute energy calibration applied to both samples that is assumed here is the one obtained via the simulated data studies described in Chapter 7. The relationship between the average, reconstructed and true (as obtained by the scintillation cubes) muon energy is parametrized in terms of a second-order polynomial over the full cube muons energy range $100 \lesssim T_{\mu}(\mathrm{MeV}) \lesssim 800$, where the difference between the average reconstructed muon energy in data and in Monte Carlo simulations is taken to be the uncertainty for each data point. The (correlated) uncertainties in the second-order polynomial parameters obtained by the fit allow one to obtain a crude estimate of energy shift, energy scale, and energy non-linearity systematic effects. A negligible energy shift, a $\delta E / E \simeq 2 \%$ energy scale factor, and a $\delta E(\mathrm{MeV}) / E(\mathrm{MeV})^{2} \simeq 4 \cdot 10^{-5}$ term describing energy non-linearities (corresponding to about $25 \mathrm{MeV}$ for $800 \mathrm{MeV}$ muons, roughly consistent with the effect seen in Fig. 7.10) are assigned as systematic uncertainties in the relative reconstructed energy response between real and simulated data. This systematic uncertainty in the reconstructed muon energy is then propagated into an uncertainty in the predicted neutrino energy reconstruction described in Chapter 7, taking into account correlations among different neutrino energy bins due to these systematic variations in energy.

\subsection{Sensitivity to $\nu_{\mu} \rightarrow \nu_{\mu}$ Oscillations}

Figure 8.1 shows the expected distribution of CCQE candidate events as a function of neutrino energy, assuming no neutrino oscillations. The errors shown are the square root of the diagonal elements of the flux, cross-section, and detector response sys- 

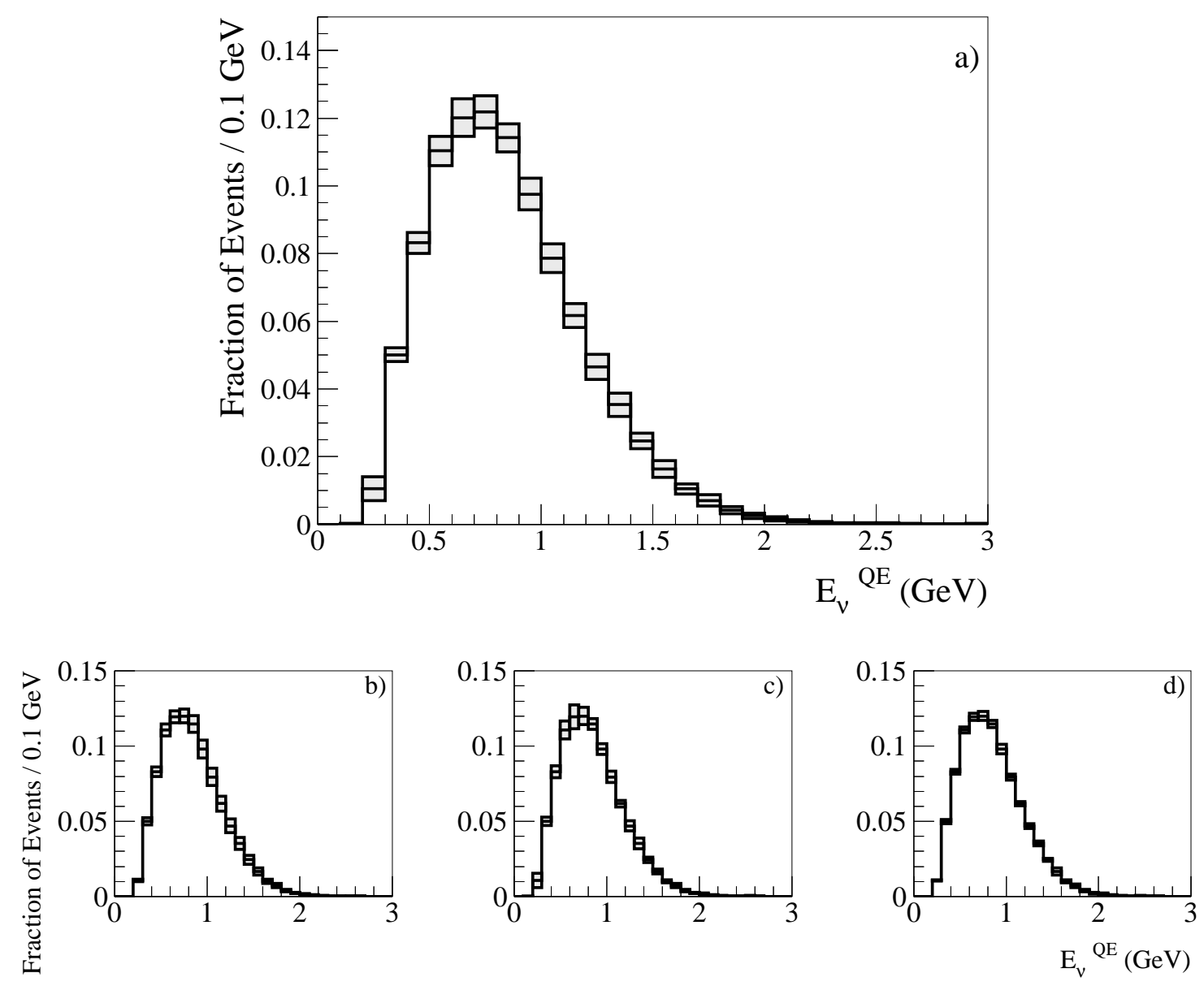

Figure 8.1: Predicted fraction of CCQE candidate events as a function of reconstructed neutrino energy $E_{\nu}^{Q E}$ assuming no neutrino oscillations, including current estimates of systematic uncertainties. In panel a), the flux, cross-section, and detector response systematic uncertainties are summed in quadrature; panels b), c), and d) show separately the flux, cross-section, and detector response systematic uncertainty contributions, respectively.

tematic error matrices discussed in the previous Section. Both the cumulative (Fig. 8.1a) and individual (Figs. 8.1b, c, d) systematic errors are shown in the Figure.

While Figure 8.1 is useful for illustrating the size of the systematic uncertainties affecting the expected CCQE neutrino energy distribution shape, it does not show the effect of correlations among neutrino energy bins, and whether or not systematic shifts can mimic an oscillation signal.

The sensitivity to neutrino oscillations is computed from Eq. 8.1, assuming that 
$N_{i}^{o b s}=N_{i, 0}^{p r e d}$, that is assuming that MiniBooNE data match the no-oscillation predictions. The only input from MiniBooNE data in the fit is in choosing a statistical sample that matches the actual number of CCQE candidate events collected, that is $5.6 \cdot 10^{4}$ events. The sensitivity is computed using a raster scan method, and the gaussian approximation is assumed. For each fixed $\Delta m^{2}$ value, the $\sin ^{2} 2 \theta_{\mu \mu}$ values that the experiment would be able to distinguish from the no-oscillation hypothesis at the $90 \%$ confidence level are the ones for which $\sin ^{2} 2 \theta_{\mu \mu}>\sin ^{2} 2 \theta_{\mu \mu, \max }$, where $\Delta \chi^{2}=\chi^{2}\left(\sin ^{2} 2 \theta_{\mu \mu, \max }, \Delta m^{2}\right)-\chi^{2}\left(\sin ^{2} 2 \theta_{\mu \mu}=0\right)=2.70$.

In order to understand how the current estimates of the various uncertainties on the predicted CCQE yields affect the MiniBooNE $\nu_{\mu}$ disappearance sensitivity, we show in Fig. 8.2 how the sources of systematic uncertainties affect the MiniBooNE sensitivity separately. Five sensitivity curves are given in Fig. 8.2, corresponding to no systematic uncertainties, only flux uncertainties, only cross-section uncertainties, only detector response uncertainties, and flux+cross-section+detection uncertainties. All curves include statistical errors corresponding to the collected CCQE data sample of $5.6 \cdot 10^{4}$ events. It is apparent from Fig. 8.2 that the sensitivity to muon neutrino disappearance at MiniBooNE is systematics-limited. Given the current uncertainty estimates, the flux contribution is the dominant factor in limiting the sensitivity of this measurement. Full detector response uncertainties, not included in Fig. 8.2, may have a significant impact on the sensitivity curve.

If systematic errors were negligible, a MiniBooNE shape-only analysis would be sensitive to muon neutrino disappearance in the $0.1 \lesssim \Delta m^{2}\left(e V^{2}\right) \lesssim 30$ range. This range is determined by the $L / E$ distribution of the experiment $(L \simeq 0.5 \mathrm{~km}$ is the distance between the neutrino production and detection locations), the CCQE sample statistics, and the neutrino energy resolution. 


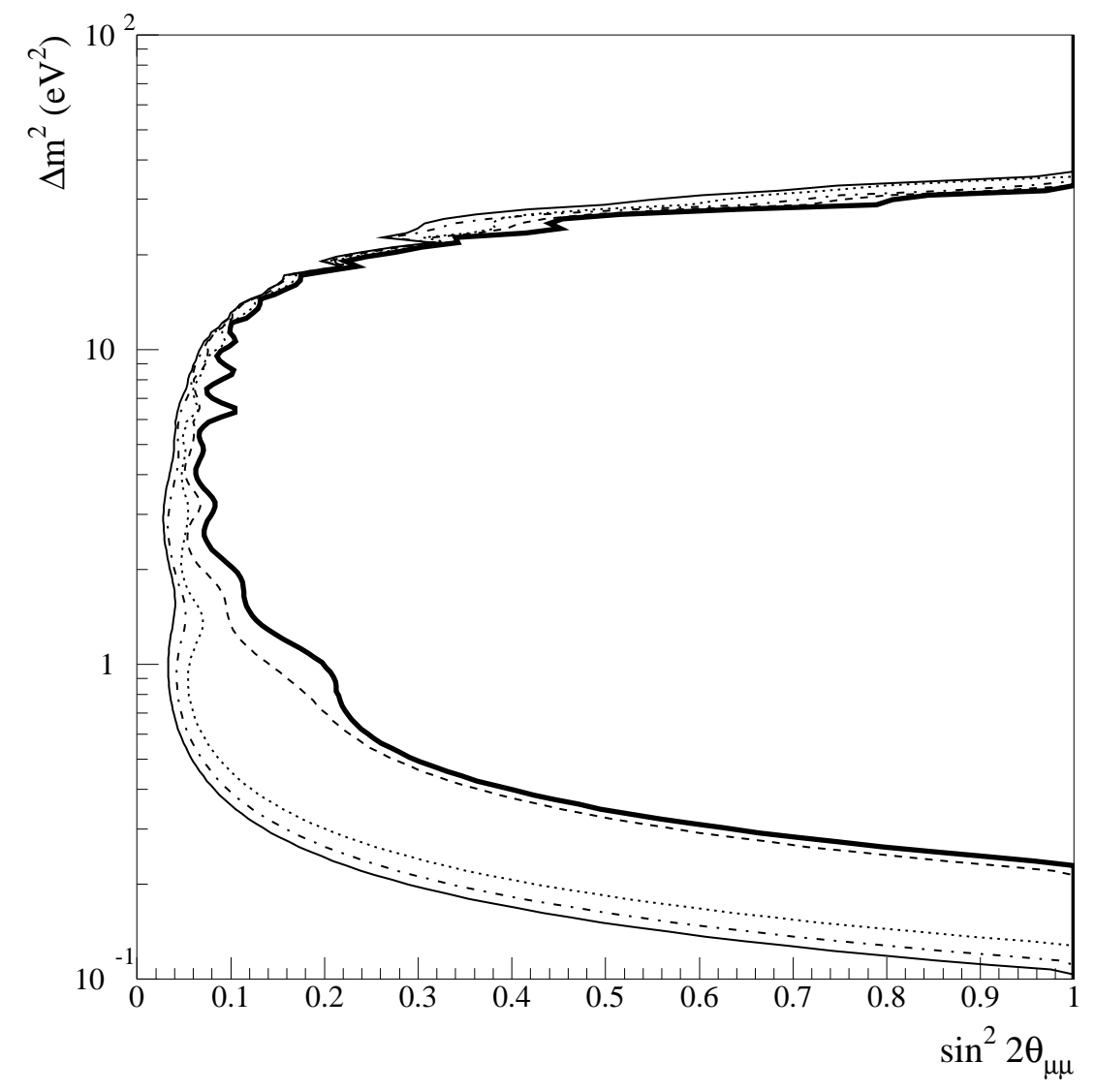

Figure 8.2: The thick solid curve shows the MiniBooNE sensitivity to $\nu_{\mu}$ disappearance given in Fig. 8.4, considering the current understanding of all systematic uncertainties. The thin lines show sensitivity curves that would be obtained with no systematic uncertainties (solid), only flux systematic uncertainties (dashed), only cross-section systematic uncertainties (dot-dashed), and only detection systematic uncertainties (dotted). All sensitivity curves include the statistical error of the data sample corresponding to $5.6 \cdot 10^{4}$ events.

\subsection{Comparing the CCQE Results with Predic- tions}

In this Section, we compare the CCQE observations with the no-oscillation predictions in terms of both the shape of the neutrino energy distribution, and in terms of the overall neutrino normalization rate. 

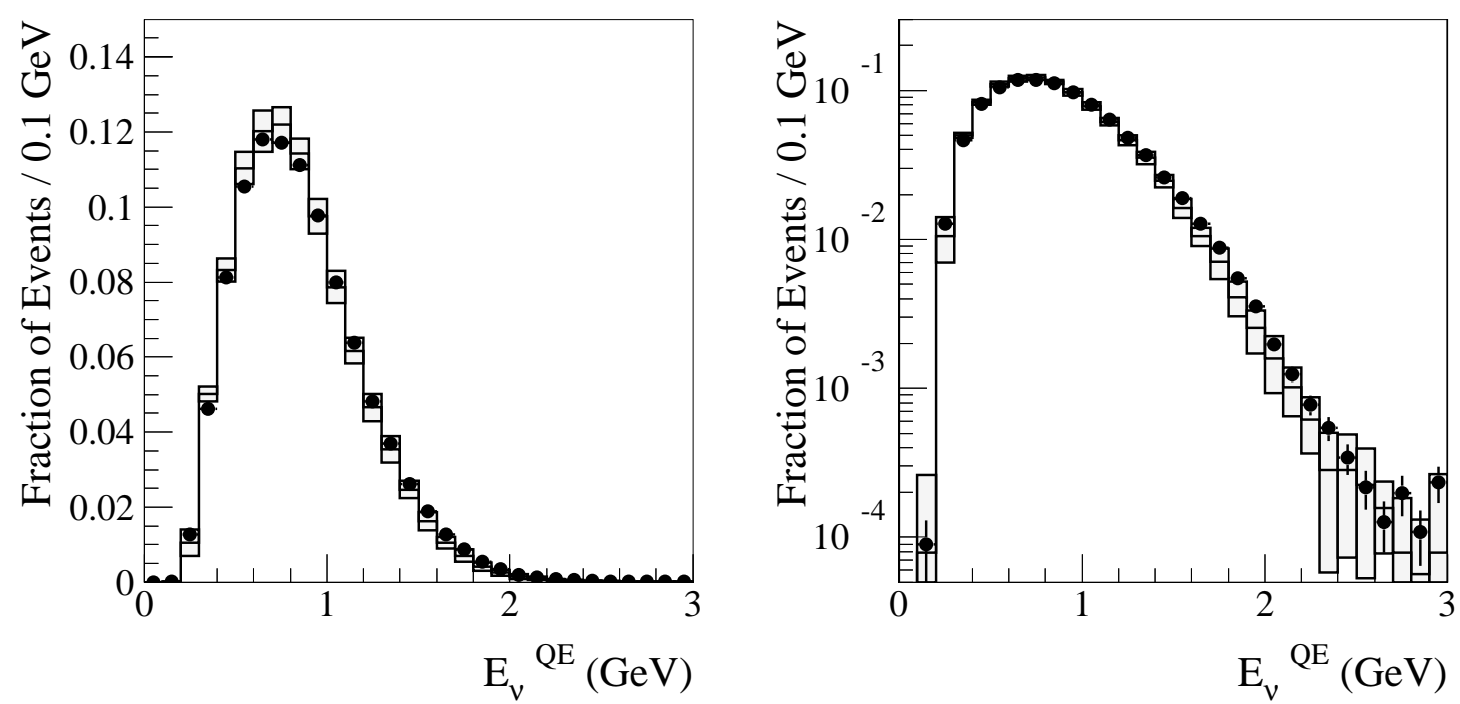

Figure 8.3: Fraction of CCQE candidate events as a function of reconstructed neutrino energy $E_{\nu}^{Q E}$, shown on a linear (left panel) and logarithmic (right panel) scale. The points show the observed distribution with statistical errors only. The light gray boxes show the predicted distribution assuming no neutrino oscillations, including current estimates of flux, cross-section, and detector response systematic uncertainties.

The observed neutrino energy distribution is compared with the shape predicted assuming no neutrino oscillations in Fig. 8.3, on both a linear and a logarithmic scale. Both distributions in the Figure are relatively normalized, and statistical and preliminary systematic uncertainty estimates are included, as previously defined. The shapes of the two curves are in qualitative agreement.

From Eq. 8.2, the overall CCQE rate normalization observed in the data can also be compared to predictions, from the known number of nuclear targets in the MiniBooNE detector and the measured number of protons on target. The measured number of CCQE candidate events per proton on target is:

$$
\left(\frac{N_{\mathrm{CCQE}}}{N_{\mathrm{pot}}}\right)^{\mathrm{obs}} \simeq 1.48 \cdot 10^{-16}
$$


where $N_{\mathrm{CCQE}} \simeq 5.58 \cdot 10^{4}$ is the number of CCQE candidate events, $N_{\text {pot }} \simeq 3.76 \cdot 10^{20}$ is the number of protons on target collected over the same time period 2 . The number of protons on target is obtained from a beam detector device located immediately upstream of the MiniBooNE target, and is expected to be accurate to within a few percent level.

The corresponding predicted number is given by:

$$
\left(\frac{N_{\mathrm{CCQE}}}{N_{\text {pot }}}\right)^{\text {pred }}=\epsilon_{\text {prescale }} \cdot \epsilon_{\mathrm{CCQE}}\left(\frac{N_{\nu}}{N_{\text {pot }}}\right)^{\text {pred }} \simeq 0.93 \cdot 10^{-16}
$$

where $\left(N_{\nu} / N_{\text {pot }}\right)^{\text {pred }} \simeq 1.90 \cdot 10^{-15}$ is the number of neutrino interactions of all types per proton on target and within the full MiniBooNE detector volume of $610 \mathrm{~cm}$ in radius, given by the flux and cross-section predictions, $\epsilon_{\text {prescale }}=0.5$ is a prescale factor applied to the CCQE sample in this analysis, and $\epsilon_{\mathrm{CCQE}} \simeq 0.0978$ is the $\mathrm{CCQE}$ event selection efficiency. The CCQE event selection efficiency is obtained from Monte Carlo studies. In order to simulate the effect of cosmic ray muons on the selection efficiency, the detector PMT hit distribution of the Monte Carlo sample was overlaid with the detector activity recorded by random triggers in time, and reconstruction and event selection algorithms are then applied to this merged data stream. Cosmic rays coincident in time with neutrino-induced interactions are expected to reduce the overall CCQE selection efficiency by about 15-20\%, as can be deduced by comparing the efficiency value quoted here with the one given in Chapter 7.

The same uncertainties affecting the energy shape of the flux and cross-section predictions, assumed in the sensitivity curve of Fig. 8.2, can also be used to estimate the overall rate normalization uncertainty. The results are given in Tab. 8.1. The flux and cross-section uncertainties contribute about equally to the expected $17 \%$ normalization rate uncertainty. This result is in contrast with the energy shape results

\footnotetext{
${ }^{2}$ Run numbers $8,235 \leq$ Run $\leq 8,348$ and $8,953 \leq$ Run $\leq 9,138$ are excluded from the proton counting over the period $3,000 \leq$ Run $\leq 10,493$, since neutrino interactions occurring during those runs do not pass the data quality requirements applied in this analysis.
} 


\begin{tabular}{|c|c|r|}
\hline Category & Type & $\begin{array}{r}\text { Normalization } \\
\text { uncertainty }(\%)\end{array}$ \\
\hline Flux & $\pi^{+}$production & 10.0 \\
& p-Be inelastic cross-section & 3.7 \\
& $K^{+}$production & 0.9 \\
& secondaries hadronic model & 0.8 \\
Flux total & Fermi gas model & 10.8 \\
\hline Cross-section & quasi-elastic axial mass & 10.8 \\
& single- $\pi$ axial mass & 6.6 \\
& $\Delta N \rightarrow N N$ rate & 3.4 \\
& coherent $\pi$ production & 1.8 \\
& other & 1.4 \\
Cross-section total & & 1.0 \\
\hline Flux + cross-section total & & 13.3 \\
\hline
\end{tabular}

Table 8.1: Predicted contributions to the systematic uncertainty in the overall rate normalization for the MiniBooNE CCQE candidate events sample over the energy range $0<E_{\nu}^{Q E}(G e V)<3$, from various flux and cross-section uncertainties. No systematic uncertainties on the CCQE event selection are included.

presented in the previous Section, and indicate that cross-section uncertainties tend to contribute in a more fully correlated way among different neutrino energy bins, compared to flux uncertainties. The dominant flux contribution is the $\pi^{+}$production uncertainty in proton-beryllium interactions; the dominant cross-section contributions are the Fermi gas model and quasi-elastic axial mass uncertainties.

Overall, the $17 \%$ normalization uncertainty derived for the CCQE rate prediction does not cover the $\simeq 60 \%$ difference between observations and predictions, and cast some doubt into the derivation of the CCQE predictions and their associated uncertainties. For this reason, and for the lack of complete detector response systematic uncertainties, neutrino oscillations cannot be constrained in a more quantitative way at this stage. 


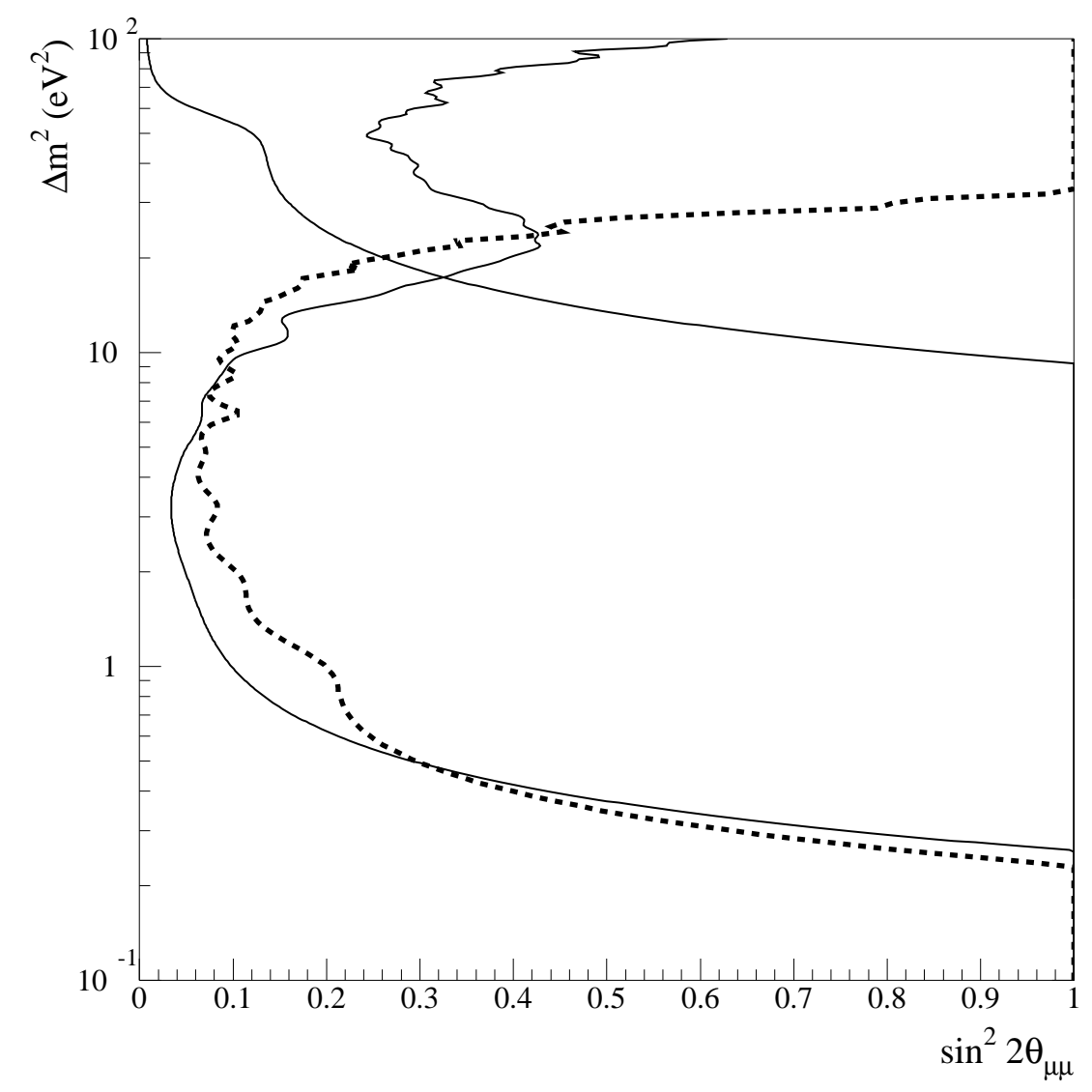

Figure 8.4: Comparison between the preliminary MiniBooNE sensitivity to muon neutrino disappearance (dashed curve), with the the upper limits from the CCFR84 (at high $\Delta m^{2}$ and CDHS (at lower $\Delta m^{2}$ ) experiments, shown by solid curves. The MiniBooNE sensitivity assumes 5.6 $10^{4}$ CCQE candidate events, together with flux, cross-section, and detector response systematic uncertainties discussed in the text. All curves assume a 90\% confidence level.

\subsection{Toward Muon Neutrino Disappearance Results}

Figure 8.4 shows the $90 \%$ confidence level MiniBooNE sensitivity to $\nu_{\mu}$ disappearance assuming the current understanding of systematic uncertainties, by looking for energy-dependent distortions in the observed distribution of CCQE events compared to the no-oscillation predictions, and without using the overall rate normalization information. Also shown in Fig. 8.4 are the existing upper limits on $\nu_{\mu}$ disappearance from past short-baseline oscillation experiments discussed in Chapter 2; CCFR84 and 
CDHS. The preliminary expectation for the MiniBooNE sensitivity to muon neutrino disappearance is similar to the oscillation sensitivity achieved by previous experiments.

The preliminary MiniBooNE sensitivity curve in Fig. 8.4 is expected to be a reasonable representation of the final sensitivity of the experiment, under the assumptions that: (1) the current observed-to-predicted rate normalization ratio discrepancy will be proven to be due to a currently unsimulated effect that is independent of neutrino energy and neutrino interaction type, (2) the detector response systematic uncertainties affecting the CCQE event selection will be shown to be negligible compared to the uncertainties presently considered. Both statements still need to be proven. Concerning the overall rate normalization, a careful scrutiny of the neutrino rate predictions is underway, and results from all MiniBooNE neutrino data samples are being compared. Concerning the detector response uncertainties, table-top measurements on light production and propagation in mineral oil are being used as inputs to the detector response expectations and uncertainty estimates, and the resulting detector optical model description is being confronted with the MiniBooNE calibration samples. Moreover, results from the HARP hadron production experiment may allow to reduce the flux systematic uncertainties, and therefore improve the MiniBooNE sensitivity to muon neutrino disappearance.

Under the sensitivity assumptions presented in Fig. 8.4, the MiniBooNE experiment is expected to provide a significant contribution toward the search for sterile neutrinos, and may be able to address (either alone, or in combination with the CCFR84 and CDHS results) oscillation parameter regions that include the range favored by sterile neutrino models (see Chapter 2).

As an example, in Figure 8.5 we show the expected constraints in the oscillation parameter space $\left(\sin ^{2} 2 \theta_{\mu \mu}, \Delta m^{2}\right)$ that would be obtained at MiniBooNE if neutrino oscillations were present, and specified by the parameters $\left(\sin ^{2} \theta_{\mu \mu}=0.3, \Delta m^{2}=\right.$ $0.92 \mathrm{eV}^{2}$ ). Those parameters are chosen because currently allowed by combined fits 


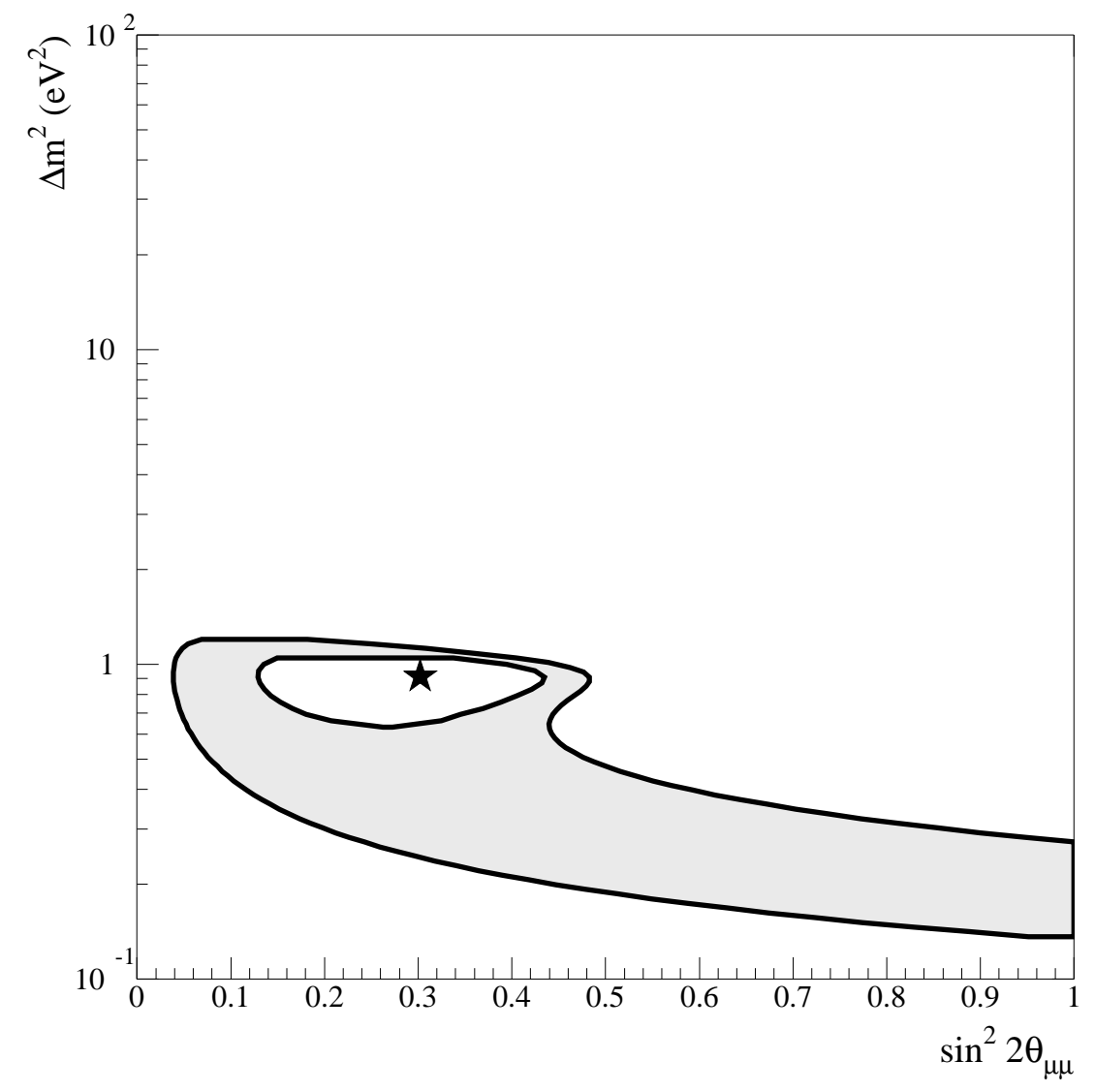

Figure 8.5: MiniBooNE sensitivity to neutrino mass and mixing parameters for a simulated oscillation signal currently allowed in $(3+1)$ sterile neutrino models, $\left(\sin ^{2} \theta_{\mu \mu}=0.3, \Delta m^{2}=\right.$ $0.92 \mathrm{eV}^{2}$ ). The allowed regions shown are at $68 \%$ and $90 \%$ confidence level, and assumes preliminary systematic uncertainties and a total data sample of $5.6 \cdot 10^{4}$ CCQE candidate events.

of $(3+1)$ sterile neutrino models, as discussed in Chapter 2 . Similarly, and also as discussed in Chapter 2, MiniBooNE is expected to have some sensitivity, and possibly to measure, the neutrino mass and mixing parameters presently allowed by $(3+2)$ sterile neutrino models. 


\section{Conclusions}

In recent years, neutrino experiments have provided convincing evidence that neutrinos are massive particles, and that oscillations among the three distinct neutrino flavors participating in the weak interactions occur. Furthermore, the LSND evidence for neutrino oscillations may point to the existence of light sterile neutrino species, that is neutral leptons with no standard weak couplings, and to active-to-sterile neutrino oscillations.

The phenomenology of sterile neutrino models explaining the LSND result is extensively studied, and motivates the search for sterile neutrinos with the Fermilab Booster Neutrino Experiment (MiniBooNE).

This thesis describes the necessary ingredients for a sterile neutrino search via the disappearance of muon neutrinos, possibly due to muon-to-sterile neutrino oscillations. This effect may be observable as an energy-dependent distortion in the predicted unoscillated muon neutrino energy spectrum. In particular, the work done to estimate the muon neutrino flux predictions at the MiniBooNE detector, and the selection and characterization of charged-current, quasi-elastic interactions of muon neutrinos $\left(\nu_{\mu} n \rightarrow \mu^{-} p\right)$, is emphasized.

The uncertainties affecting the experiment's sensitivity to muon neutrino disappearance are reviewed, and reveal the possibility to explore unchartered territory in the neutrino mass and mixing parameter space that may be responsible for activeto-sterile neutrino oscillations. Preliminary comparisons between observations and predictions are presented, as a first step toward a muon neutrino disappearance result. 


\section{Bibliography}

[1] J. Chadwick, Proc. R. Soc. London (A) 136, 692 (1932).

[2] C. D. Anderson, Phys. Rev. 43, 491 (1933).

[3] E. Fermi, Z. Phys. 88, 161 (1934).

[4] E. Majorana, Nuovo Cim. 14, 171 (1937).

[5] C. L. Cowan, F. Reines, F. B. Harrison, H. W. Kruse and A. D. McGuire, Science 124, 103 (1956).

[6] T. D. Lee and C. N. Yang, Phys. Rev. 104, 254 (1956).

[7] C. S. Wu, E. Ambler, R. W. Hayward, D. D. Hoppes and R. P. Hudson, Phys. Rev. 105, 1413 (1957).

[8] T. D. Lee and C. N. Yang, Phys. Rev. 105, 1671 (1957).

[9] M. Goldhaber, L. Grodzins and A. W. Sunyar, Phys. Rev. 109, 1015 (1958).

[10] B. Pontecorvo, Sov. Phys. JETP 6, 429 (1957) [Zh. Eksp. Teor. Fiz. 33, 549 (1957)].

[11] M. Gell-Mann and A. Pais, Phys. Rev. 97, 1387 (1955).

[12] E. C. G. Sudarshan and R. e. Marshak, Phys. Rev. 109, 1860 (1958);

R. P. Feynman and M. Gell-Mann, Phys. Rev. 109, 193 (1958). 
[13] G. Danby, J. M. Gaillard, K. Goulianos, L. M. Lederman, N. Mistry, M. Schwartz and J. Steinberger, Phys. Rev. Lett. 9, 36 (1962).

[14] Z. Maki, M. Nakagawa and S. Sakata, Prog. Theor. Phys. 28, 870 (1962).

[15] J. H. Christenson, J. W. Cronin, V. L. Fitch and R. Turlay, Phys. Rev. Lett. 13, 138 (1964).

[16] M. Kobayashi and T. Maskawa, Prog. Theor. Phys. 49, 652 (1973).

[17] A. D. Sakharov, Pisma Zh. Eksp. Teor. Fiz. 5, 32 (1967) [JETP Lett. 5, 24 (1967 SOPUA,34,392-393.1991 UFNAA,161,61-64.1991)].

[18] S. L. Glashow, Nucl. Phys. 22, 579 (1961); S. Weinberg, Phys. Rev. Lett. 19, 1264 (1967); A. Salam, "Weak And Electromagnetic Interactions," in Elementary Particle Theory, Proc. 8th Nobel Symp., 367 (1968).

[19] F. Englert and R. Brout, Phys. Rev. Lett. 13, 321 (1964); P. W. Higgs, Phys. Rev. Lett. 13, 508 (1964); G. S. Guralnik, C. R. Hagen and T. W. B. Kibble, Phys. Rev. Lett. 13, 585 (1964); Y. Nambu and G. Jona-Lasinio, Phys. Rev. 122, 345 (1961); J. Goldstone, Nuovo Cim. 19, 154 (1961).

[20] F. J. Hasert et al. [Gargamelle Neutrino Collaboration], Phys. Lett. B 46, 138 (1973).

[21] G. Arnison et al. [UA1 Collaboration], Phys. Lett. B 122, 103 (1983);

G. Arnison et al. [UA1 Collaboration], Phys. Lett. B 126, 398 (1983).

[22] R. J. Davis, D. S. Harmer and K. C. Hoffman, Phys. Rev. Lett. 20, 1205 (1968).

[23] B. T. Cleveland et al., Astrophys. J. 496, 505 (1998).

[24] V. N. Gribov and B. Pontecorvo, Phys. Lett. B 28, 493 (1969). 
[25] K. Hirata et al. [KAMIOKANDE-II Collaboration], Phys. Rev. Lett. 58, 1490 (1987); R. M. Bionta et al., Phys. Rev. Lett. 58, 1494 (1987).

[26] K. S. Hirata et al. [KAMIOKANDE-II Collaboration], Phys. Lett. B 205, 416 (1988).

[27] Y. Fukuda et al. [Super-Kamiokande Collaboration], Phys. Rev. Lett. 81, 1562 (1998) [arXiv:hep-ex/9807003].

[28] K. Kodama et al. [DONUT Collaboration], Phys. Lett. B 504, 218 (2001) [arXiv:hep-ex/0012035].

[29] M. L. Perl et al., Phys. Rev. Lett. 35, 1489 (1975).

[30] D. Decamp et al. [ALEPH Collaboration], Phys. Lett. B 231, 519 (1989).

[31] S. P. Mikheev and A. Y. Smirnov, Sov. J. Nucl. Phys. 42, 913 (1985) [Yad. Fiz. 42, 1441 (1985)].

[32] L. Wolfenstein, Phys. Rev. D 20, 2634 (1979).

[33] K. S. Hirata et al. [KAMIOKANDE-II Collaboration], Phys. Rev. Lett. 63, 16 (1989).

[34] A. I. Abazov et al., Phys. Rev. Lett. 67, 3332 (1991).

[35] P. Anselmann et al. [GALLEX Collaboration], Phys. Lett. B 285, 376 (1992).

[36] S. Fukuda et al. [Super-Kamiokande Collaboration], Phys. Rev. Lett. 86, 5651 (2001) [arXiv:hep-ex/0103032]; Q. R. Ahmad et al. [SNO Collaboration], Phys. Rev. Lett. 87, 071301 (2001) [arXiv:nucl-ex/0106015].

[37] C. Athanassopoulos et al. [LSND Collaboration], Phys. Rev. Lett. 77, 3082 (1996) [arXiv:nucl-ex/9605003]; A. Aguilar et al. [LSND Collaboration], Phys. Rev. D 64, 112007 (2001) [arXiv:hep-ex/0104049]. 
[38] M. H. Ahn et al. [K2K Collaboration], Phys. Rev. Lett. 90, 041801 (2003) [arXiv:hep-ex/0212007].

[39] K. Eguchi et al. [KamLAND Collaboration], Phys. Rev. Lett. 90, 021802 (2003) [arXiv:hep-ex/0212021].

[40] B. Kayser, arXiv:hep-ph/0211134.

[41] C. Giunti and M. Laveder, arXiv:hep-ph/0310238.

[42] Y. Ashie et al. [Super-Kamiokande Collaboration], Phys. Rev. Lett. 93, 101801 (2004) [arXiv:hep-ex/0404034].

[43] M. Apollonio et al., Eur. Phys. J. C 27, 331 (2003) [arXiv:hep-ex/0301017].

[44] Q. R. Ahmad et al. [SNO Collaboration], Phys. Rev. Lett. 89, 011301 (2002) [arXiv:nucl-ex/0204008].

[45] J. N. Bahcall, M. H. Pinsonneault and S. Basu, Astrophys. J. 555, 990 (2001) [arXiv:astro-ph/0010346].

[46] T. Araki et al. [KamLAND Collaboration], arXiv:hep-ex/0406035.

[47] M. C. Gonzalez-Garcia and C. Pena-Garay, Phys. Rev. D 68, 093003 (2003) [arXiv:hep-ph/0306001].

[48] S. c. Freedman and B. c. Kayser, "The neutrino matrix," arXiv:physics/0411216.

[49] B. Armbruster et al. [KARMEN Collaboration], Phys. Rev. D 65, 112001 (2002) [arXiv:hep-ex/0203021].

[50] P. Astier et al. [NOMAD Collaboration], Phys. Lett. B 570, 19 (2003) [arXiv:hep-ex/0306037].

[51] Y. Declais et al., Nucl. Phys. B 434, 503 (1995). 
[52] I. E. Stockdale et al., Phys. Rev. Lett. 52, 1384 (1984).

[53] F. Dydak et al., Phys. Lett. B 134, 281 (1984).

[54] P. Astier et al. [NOMAD Collaboration], Nucl. Phys. B 611, 3 (2001) [arXiv:hep-ex/0106102].

[55] E. Eskut et al. [CHORUS Collaboration], Phys. Lett. B 497, 8 (2001).

[56] E. D. Church, K. Eitel, G. B. Mills and M. Steidl, Phys. Rev. D 66, 013001 (2002) [arXiv:hep-ex/0203023].

[57] Y. Farzan and A. Y. Smirnov, Phys. Lett. B 557, 224 (2003) [arXiv:hep-ph/0211341]; Y. Farzan, O. L. G. Peres and A. Y. Smirnov, Nucl. Phys. B 612, 59 (2001) [arXiv:hep-ph/0105105].

[58] V. M. Lobashev et al., Nucl. Phys. Proc. Suppl. 91, 280 (2001); J. Bonn et al., Nucl. Phys. Proc. Suppl. 91, 273 (2001).

[59] A. Osipowicz et al. [KATRIN Collaboration], arXiv:hep-ex/0109033; L. Bornschein [KATRIN Collaboration], eConf C030626, FRAP14 (2003) [arXiv:hep-ex/0309007].

[60] S. Eidelman et al. [Particle Data Group Collaboration], Phys. Lett. B 592, 1 (2004).

[61] K. N. Abazajian, Astropart. Phys. 19, 303 (2003) [arXiv:astro-ph/0205238].

[62] G. L. Fogli, E. Lisi, A. Marrone, A. Melchiorri, A. Palazzo, P. Serra and J. Silk, Phys. Rev. D 70, 113003 (2004) [arXiv:hep-ph/0408045].

[63] R. Foot and R. R. Volkas, Phys. Rev. Lett. 75, 4350 (1995) [arXiv:hep-ph/9508275]. 
[64] G. Gelmini, S. Palomares-Ruiz and S. Pascoli, Phys. Rev. Lett. 93, 081302 (2004) [arXiv:astro-ph/0403323]; S. Hannestad, Phys. Rev. D 70, 043506 (2004) [arXiv:astro-ph/0403291].

[65] J. F. Beacom, N. F. Bell and S. Dodelson, Phys. Rev. Lett. 93, 121302 (2004) [arXiv:astro-ph/0404585].

[66] K. Abazajian, N. F. Bell, G. M. Fuller and Y. Y. Y. Wong, arXiv:astro-ph/0410175.

[67] A. D. Dolgov, S. H. Hansen, S. Pastor, S. T. Petcov, G. G. Raffelt and D. V. Semikoz, Nucl. Phys. B 632, 363 (2002) [arXiv:hep-ph/0201287].

[68] http://www-boone.fnal.gov/publicpages/runplan.ps.gz

[69] M. Sorel and J. M. Conrad, Phys. Rev. D 66, 033009 (2002) [arXiv:hep-ph/0112214].

[70] M. Sorel, J. M. Conrad and M. Shaevitz, Phys. Rev. D 70, 073004 (2004) [arXiv:hep-ph/0305255].

[71] A. Aguilar-Arevalo, V. Barger, J. M. Conrad, M. Shaevitz, M. Sorel, K. Whisnant, "CP violation in $(3+2)$ sterile neutrino models," in preparation.

[72] P. Brâemaud, Markov chains: Gibbs fields, Monte Carlo simulation, and queues, Springer, New York, 1999.

[73] N. Metropolis, A. W. Rosenbluth, M. N. Rosenbluth, A. H. Teller and E. Teller, J. Chem. Phys. 21, 1087 (1953).

[74] I. Stancu et al. [MiniBooNE collaboration], "Technical Design Report for the $8 \mathrm{GeV}$ Beam," http://www-boone.fnal.gov/publicpages/8gevtdr_2.0.ps.gz 
[75] I. Stancu et al. [MiniBooNE collaboration], "Technical Design Report for the MiniBooNE Neutrino Beam," http://www-boone.fnal.gov/publicpages/target_tdr.ps.gz

[76] C. Moore et al., "Initial Operation of the Fermilab MiniBooNE Beamline", Proceedings of the 2003 Particle Accelerator Conference, http://accelconf . web.cern.ch/AccelConf/p03/PAPERS/TPPB013.PDF

[77] http://www. bartoszekeng.com/mboone/mboone.htm

[78] L. Bugel and M. Sorel, "Magnetic Field Measurements for the MiniBooNE Prototype Horn", BooNE Technical Note 34 (2001).

[79] J. Monroe, M. Shaevitz, "Estimating the Uncertainty on the Electron Neutrino Background from Intrinsic Beam Muon Decay Using the Charged Current Quasi-Elastic Data Set", BooNE Technical Note 146 (2004).

[80] S. Agostinelli et al. [GEANT4 Collaboration], Nucl. Instrum. Meth. A 506, $250(2003)$.

[81] http://wwwasd.web.cern.ch/geant4/G4UsersDocuments/Overview/html/

[82] J. Link and J. Monroe, private communication. Publication in preparation.

[83] N. V. Mokhov, FERMILAB-FN-0628 (1995).

[84] N. V. Mokhov, K. K. Gudima, C. C. James et al., FERMILAB-Conf-04/053 (2004).

[85] A. Fasso' et al., eConf C0303241, MOMT004 (2003) [arXiv:physics/0306162].

[86] "GEANT - Detector Description and Simulation Tool", CERN Program Library Long Writeup W5013, CERN, Edition March 1995, http://wwwasdoc.web.cern.ch/wwwasdoc/geant_html3/geantall.html 
[87] Y. Cho et al., Phys. Rev. D 4, 1967 (1971).

[88] I. A. Vorontsov, V. A. Ergakov, G. A. Safronov, A. A. Sibirtsev, G. N. Smirnov, N. V. Stepanov and Y. V. Trebukhovsky, ITEP-85-1983.

[89] J. V. Allaby et al., Phys. Lett. B 30, 549 (1969).

[90] G. J. Marmer and D. E. Lundquist, Phys. Rev. D 3, 1089 (1971).

[91] Y. D. Aleshin, I. A. Drabkin and V. V. Kolesnikov, ITEP-80-1977.

[92] HARP Collaboration, CERN-SPSC-2004-018.

[93] V. V. Gachurin et al., ITEP-59-1985.

[94] Lykhachev, Zh. Eksp. Teor. Fiz. 41 (1981) 39;

[95] Afonasiev, Yad. Fiz. 47 (1988) 1656.

[96] http://cernlib.web.cern.ch/cernlib/overview.html

[97] A. Sher et al., Phys. Rev. Lett. 91, 261802 (2003) [arXiv:hep-ex/0305042]; T. Alexopoulos et al. [KTeV Collaboration], Phys. Rev. D 70, 092006 (2004) [arXiv:hep-ex/0406002].

[98] S. Geer, Phys. Rev. D 57, 6989 (1998) [Erratum-ibid. D 59, 039903 (1999)] [arXiv:hep-ph/9712290].

[99] L. M. Chounet, J. M. Gaillard and M. K. Gillard, Phys. Rept. 4, 199 (1972).

[100] D. Casper, Nucl. Phys. Proc. Suppl. 112, 161 (2002) [arXiv:hep-ph/0208030].

[101] P. Lipari, M. Lusignoli and F. Sartogo, Phys. Rev. Lett. 74, 4384 (1995) [arXiv:hep-ph/9411341].

[102] W. A. Mann et al., Phys. Rev. Lett. 31, 844 (1973).

[103] S. J. Barish et al., Phys. Rev. D 19, 2521 (1979). 
[104] N. J. Baker, P. L. Connolly, S. A. Kahn, M. J. Murtagh, R. B. Palmer, N. P. Samios and M. Tanaka, Phys. Rev. D 25, 617 (1982).

[105] D. MacFarlane et al., Z. Phys. C 26, 1 (1984).

[106] P. E. Renton, Electroweak Interactions, Cambridge University Press, Cambridge, 1990.

[107] E. .D. Commins, P. H. Bucksbaum, Weak interactions of leptons and quarks, Cambridge University Press, Cambridge, 1983.

[108] C. H. Llewellyn Smith, Phys. Rept. 3, 261 (1972).

[109] E. A. Paschos and J. Y. Yu, Phys. Rev. D 65, 033002 (2002) [arXiv:hep-ph/0107261].

[110] D. Rein and L. M. Sehgal, Annals Phys. 133, 79 (1981).

[111] R. P. Feynman, M. Kislinger and F. Ravndal, Phys. Rev. D 3, 2706 (1971).

[112] D. Rein and L. M. Sehgal, Nucl. Phys. B 223, 29 (1983).

[113] A. Bodek and U. K. Yang, arXiv:hep-ex/0308007.

[114] M. Gluck, E. Reya and A. Vogt, Eur. Phys. J. C 5, 461 (1998) [arXiv:hep-ph/9806404].

[115] S. Gasiorowicz, Elementary Particle Physics, John Wiley and Sons, New York, 1966.

[116] M. Nowakowski, E. A. Paschos and J. M. Rodriguez, arXiv:physics/0402058.

[117] D. Griffiths, Introduction to Elementary Particles, John Wiley and Sons, New York, 1987.

[118] K. F. Liu, S. J. Dong, T. Draper and W. Wilcox, Phys. Rev. Lett. 74, 2172 (1995) [arXiv:hep-lat/9406007]. 
[119] P. E. Bosted, Phys. Rev. C 51, 409 (1995).

[120] S. J. Barish et al., Phys. Rev. D 16, 3103 (1977).

[121] N. J. Baker et al., Phys. Rev. D 23, 2499 (1981).

[122] R. A. Smith and E. J. Moniz, Nucl. Phys. B 43, 605 (1972) [Erratum-ibid. B 101, $547(1975)]$.

[123] J. S. Bell and C. H. Llewellyn Smith, Nucl. Phys. B 28, 317 (1971).

[124] S. K. Singh and E. Oset, Nucl. Phys. A 542, 587 (1992).

[125] S. Bonetti et al., Nuovo Cim. A 38, 260 (1977).

[126] J. Brunner et al. [SKAT Collaboration], Z. Phys. C 45, 551 (1990).

[127] S. V. Belikov et al., Yad. Fiz. 35, 59 (1982).

[128] S. V. Belikov et al., Z. Phys. A 320, 625 (1985).

[129] D. Ashery, I. Navon, G. Azuelos, H. K. Walter, H. J. Pfeiffer and F. W. Schleputz, Phys. Rev. C 23, 2173 (1981).

[130] I. Navon et al., Phys. Rev. C 28, 2548 (1983);

[131] I. Stancu et al. [MiniBooNE collaboration], "The Miniboone Detector Technical Design Report," FERMILAB-TM-2207.

[132] B. Fleming, D. Smith, "MiniBooNE Photomultiplier Tube Testing", BooNE Technical Note 30 (2002).

[133] R. L. Imlay et al., "Study of the Angular Reconstruction of Muons in MiniBooNE Using the Muon Tracker", BooNE Technical Note 99 (2003).

[134] R. L. Imlay et al., "Measuring the Energy of Muons in MiniBooNE with the Cubes", BooNE Technical Note 106 (2004). 
[135] H. H. Meyer, "Index of Refraction of Marcol 7", BooNE Technical Note 90 (2003).

[136] N. Suwonjandee, R. Johnson, "Mineral Oil Index of Refraction", http://www.physics.uc.edu/ johnson/Boone/oil_page/refraction/

[137] B. C. Brown et al. [the MiniBooNE collaboration], "Study of scintillation, fluorescence and scattering in mineral oil for the MiniBooNE neutrino detector," FERMILAB-CONF-04-282-E; Prepared for 2004 IEEE Nuclear Science Symposium and Medical Imaging Conference (NSS / MIC), Rome, Italy, 16-22 Oct 2004.

[138] A. O. Bazarko et al., "Studies of Scattering in Marcol 7 Mineral Oil", BooNE Technical Note 144 (2004).

[139] The technical specifications for the Hamamatsu R5912 photomultiplier tube can be downloaded from:

http://www.datasheetarchive.com/HamamatsuCorporation4.html

[140] R. B. Patterson, "The PMT Charge Calculation”, BooNE Technical Note 83 (2003).

[141] Y. Liu and I. Stancu, "The MiniBooNE Charge Likelihoods and Light Scattering in Michel Electron Events", BooNE Technical Note 126 (2004).

[142] Y. Liu and I. Stancu, "Toward the MiniBooNE Charge Likelihood", BooNE Technical Note 100 (2003).

[143] I. Stancu, "The Single-PE Time Resolution with Ryan Patterson's QT-Algorithms", BooNE Technical Note 81 (2003).

[144] I. Stancu, "An Introduction to the Maximum Likelihood Event Reconstruction in MiniBooNE", BooNE Technical Note 50 (2002). 
[145] Y. Liu and I. Stancu, "In Situ Speed of Light Measurements at $\lambda=400 \mathrm{~nm}$ and $\lambda=440$ nm", BooNE Technical Note 125 (2004).

[146] A. Aguilar-Arevalo, "Neutrino Energy Reconstruction Studies for numu Quasi-Elastic Events", BooNE Technical Note 97 (2003).

[147] J. L. Raaf [BooNE Collaboration], arXiv:hep-ex/0408015.

[148] Y. Liu and I. Stancu, "The Performance of the S-Fitter Particle Identification", BooNE Technical Note 141 (2004).

[149] E. Aliu et al. [K2K Collaboration], arXiv:hep-ex/0411038. 